ERNEST $\square R L A N D Q L A W R E N C E$

BERRELEY NATU⿴NAL LABDRATGRY

$4+9 k$

Time- and State-Resolved

Spectroscopy, Diffraction, and

Circular Dichroism in Core

Photoelectron Emission from Clean and Oxygen-Covered W(110)

Ramon Xavier Ynzunza

Materials Sciences Division

October 1998

Ph.D. Thesis
PRECEIVEO

MAR 291999

OSTI 


\section{DISCLAIMER}

This document was prepared as an account of work sponsored by the United States Government. While this document is believed to contain correct information, neither the United States Government nor any agency thereof, nor The Regents of the University of California, nor any of their employees, makes any warranty, express or implied, or assumes any legal responsibility for the accuracy, completeness, or usefulness of any information, apparatus, product, or process disclosed, or represents that its use would not infringe privately owned rights. Reference herein to any specific commercial product, process, or service by its trade name, trademark, manufacturer, or otherwise, does not necessarily constitute or imply its endorsement, recommendation, or favoring by the United States Government or any agency thereof, or The Regents of the University of California. The views and opinions of authors expressed herein do not necessarily state or reflect those of the United States Government or any agency thereof, or The Regents of the University of California.

Ernest Orlando Lawrence Berkeley National Laboratory is an equal opportunity employer. 


\section{DISCLAIMER}

Portions of this document may be illegible in electronic image products. Images are produced from the best available original document. 
LBNL-42585

\title{
Time- and State-Resolved Spectroscopy, Diffraction, and Circular Dichroism in Core Photoelectron Emission from Clean and Oxygen-Covered W(110)
}

\author{
Ramon Xavier Ynzunza \\ Ph.D. Thesis \\ Department of Physics \\ University of California, Davis \\ and \\ Materials Sciences Division \\ Ernest Orlando Lawrence Berkeley National Laboratory \\ University of California \\ Berkeley, California 94720
}

October 1998

This work was supported by the Director, Office of Energy Research, Office of Basic Energy Sciences, Materials Sciences Division, of the U.S. Department of Energy under Contract No. DE-AC03-76SF00098, the Office of Naval Research under Contract No. N00014-940162, and the National Energy Research Supercomputing Center at LBNL. 
Acknowledgements

I would like to thank my wife Guadalupe Ynzunza for all the support she has given me. There were many times when I needed her inspiration and understanding, and she was always there. I would also like to thank my advisor Professor Charles S. Fadley whose advice and encouragement made completing this thesis possible. I would further like to thank Drs. G. Biino, Y. Chen, H. Daimon, R. Denecke, J. García de Abajo, A.P. Kaduwela, K. Higashiyama, J. Morais, F.J. Palomares, S. Thevuthasan, M.A. Van Hove, and C. Westphal, who have participated with me in collecting and analyzing the data. Finally, I would like to thank all of the graduate students in Professor Fadley's research group who have been major contributors to this work, in particular R.E. Couch, Y.J. Kim, P.M. Len, S.D. Ruebush, E.D. Tober, and Z. Wang.

Support for these studies was provided by the Director, Office of Energy Research, Materials Sciences Division, of the U.S. Department of Energy under Contract No. DE-AC03-76SF00098, the Office of Naval Research under contract (N00014940162), and the National Energy Research Supercomputing Center at LBNL. 


\begin{abstract}
Several aspects of core-level photoelectron emission from solid surfaces as excited by high-brightness variable-polarization synchrotron radiation have been studied with a new beamline and experimental station at the Advanced Light Source in Berkeley. These include: resolution of different chemical states and site types via high-resolution photoelectron spectroscopy (PS), the use of state-resolved photoelectron diffraction (PD) to determine local atomic geometries, and the observation and analysis of circular dichroism (CD) effects in photoelectron diffraction. These methods have been applied to clean and oxygen-exposed surfaces of W(110). Full-solid-angle photoelectron diffraction from clean $\mathrm{W}(110)$ was measured, with the surface and bulk atoms being clearly resolved. This provided a test case for R-factor structure analysis of low energy photoelectron diffraction data, with experiment compared to theoretical calculations based on multiple-scattering theory. We found a $4.5 \%$ outward relaxation of the surface W layer, in good agreement with prior measurements. We also determined the positions of oxygen atoms in a saturated (1x1) oxygen overlayer on W(110) from full-solid-angle photoelectron diffraction with chemical state resolution, arriving at an W-oxygen interplaner distance of $0.91 \pm 0.05 \AA$ and a small shift from the geometric center of the pseudo-threefold hollow sites on $\mathrm{W}(110)$ by $0.10 \mathrm{~A}$ in the [1 $\overline{1} 0]$ direction. Using timeand state-resolved measurements, we studied the adsorption kinetics of oxygen on $\mathrm{W}(110)$, finding that gas pressure is an important variable in the kind of adsorption structure formed, with low-pressure adsorption in the $10^{-9}$ torr range not leading to any
\end{abstract}


long-range ordered oxygen structures in LEED. State-resolved PS and PD measurements nonetheless permitted determining the state-by-state kinetics, as well as the atomic position of the oxygen atoms in regions of the surface where only short-range order exists. A simple Monte Carlo model was found to reproduce several features of the kinetics data. Finally, we studied circular dichroism in core photoelectron emission from (1x1) oxygen on $\mathrm{W}(110)$, comparing experiment and multiple scattering theory. We observed large differences of up to $50 \%$ in the PD patterns when polarization is changed from right to left circular polarization and these $C D$ effects are quantitatively reproduced by the calculations. 
Table of Contents:

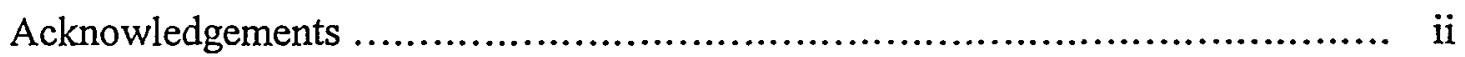

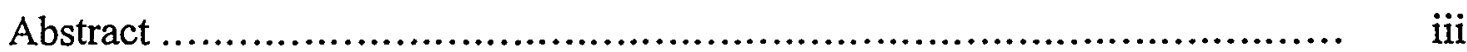

Chapter 1: General Introduction ................................................... 1

1.I. Surface Science ........................................................ 1

1.II. Photoelectron Spectroscopy and Photoelectron Diffraction ............. 2

1.III. Synchrotron Radiation .................................................. 9

1.N. Thesis Outline .................................................... 11

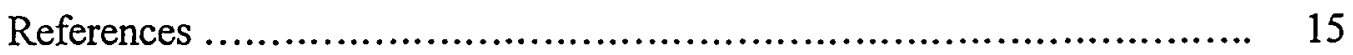

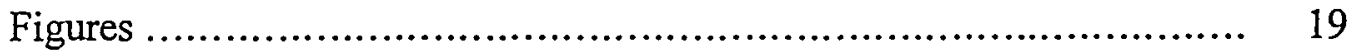

Chapter 2: Instrumentation and Data Analysis ....................................... 21

2.I. Instrumentation Development .......................................... 21

2.II. Data Analysis .......................................................... 30

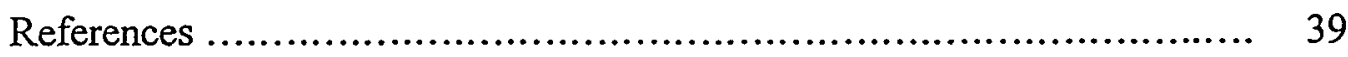

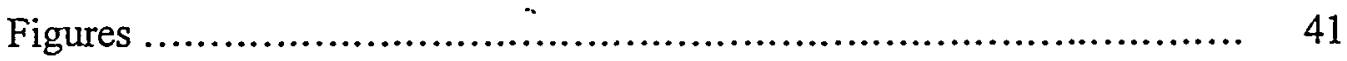

Chapter 3: Full-Solid-Angle Photoelectron Diffraction from Bulk and Surface

Atoms of Clean W(110) ................................................... 56

3.I. Introduction .......................................................... 56

3.II. Experimental Methodology ............................................ 57

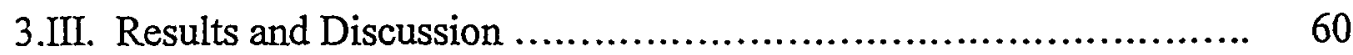

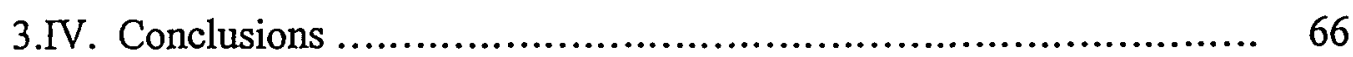

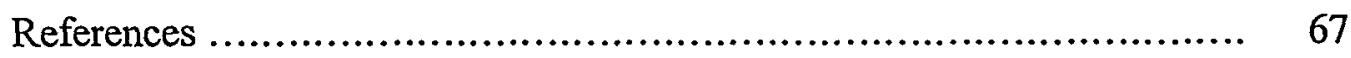

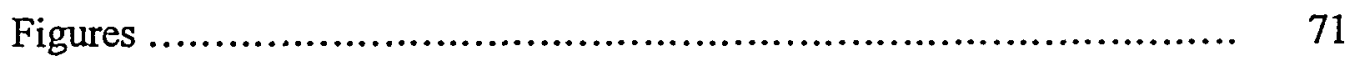

Chapter 4: Structure Determination for Saturated (1x1) Oxygen on W(110) from

Full-Solid-Angle Photoelectron Diffraction with Chemical-State

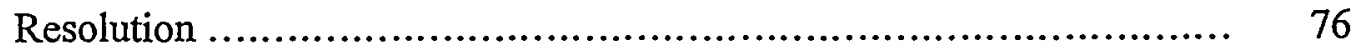

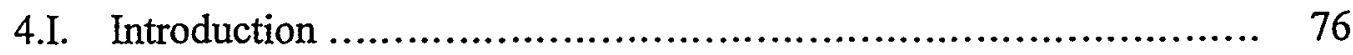

4.II. Experimental Methodology ........................................... 78

4.III. Structure Analysis and Discussion ..................................... 82

4.IV. Conclusions ............................................................ 86

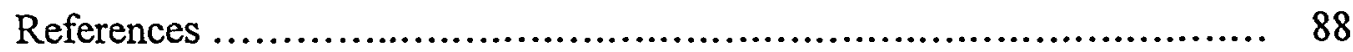

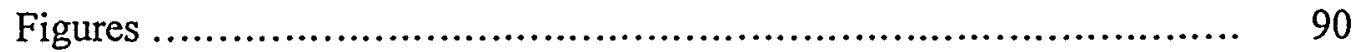


Chapter 5: Kinetics and Atomic Structure of O Adsorption on W(110) from Timeand State- Resolved Photoelectron Spectroscopy and Full-Solid-Angle Photoelectron Diffraction ................................................... 96

5.I. Introduction ...................................................... 96

5.II. Experimental Methodology ........................................... 98

5.III. Results and Discussion .............................................. 103

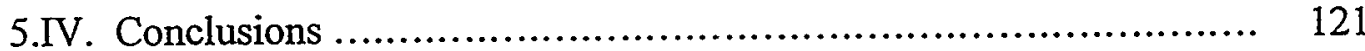

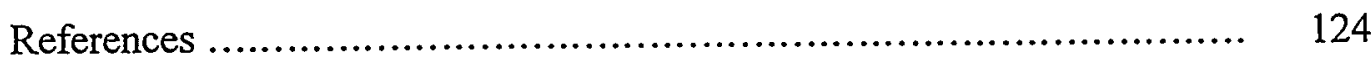

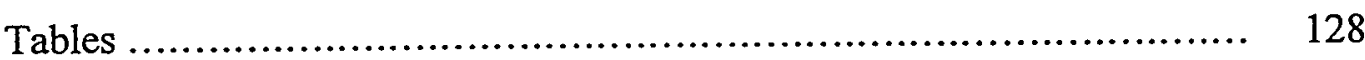

Figures ............................................................... 130

Chapter 6: Circular Dichroism in Core Photoelectron Emission from (1x1)

Oxygen on W(110): Experiment and Multiple-Scattering Theory 143

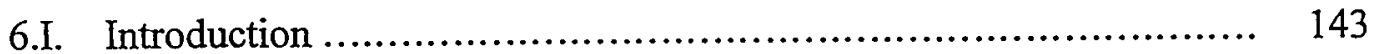

6.II. Experiment ........................................................... 145

6.III. Theoretical Background ............................................. 148

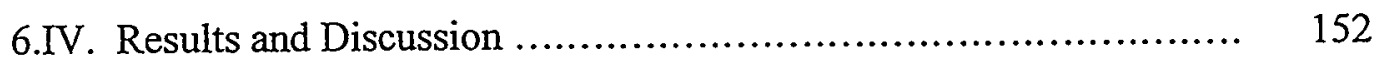

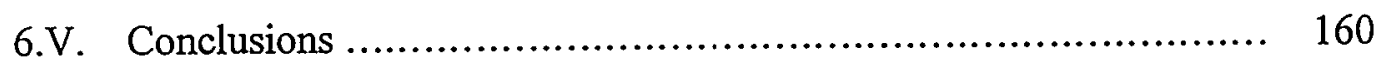

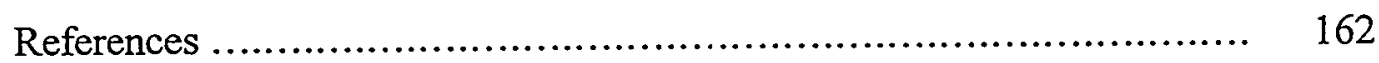

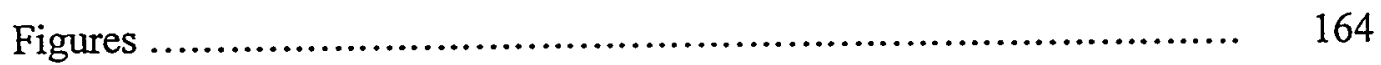

Chapter 6: Concluding Remarks .............................................. 180

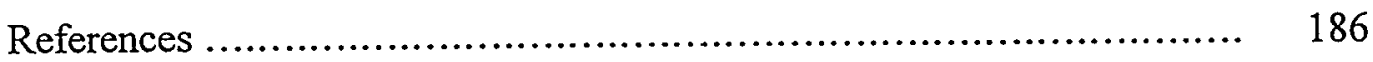




\section{Chapter 1}

\section{General Introduction}

\section{I. Surface Science}

As the dimensions of elements in integrated circuits, magnetic storage devices, and other important technologies continue to shrink into the nanometer scale, a quantitative microscopic understanding of surface phenomena becomes increasingly more important. Other technologically significant processes in which surface physics and chemistry play crucial roles are catalysis, oxidation, corrosion, adhesion, and the formation of overlayers, interfaces, and nanostructures. This dissertation deals with photoelectron spectroscopy and diffraction techniques performed with high-brightness third-generation synchrotron radiation so as to provide several new methods for the quantitative analysis of these surface phenomena. The research includes the development of unique instrumentation for such studies, applications of it to illustrative test cases to demonstrate new capabilities, and studies of surfaces for which novel conclusions concerning atomic structure, chemical kinetics, and circular dichroism effects can be drawn. 


\section{II. Photoelectron Spectroscopy and Photoelectron Diffraction}

In photoelectron spectroscopy (PS), a sample is exposed to a source of monoenergetic photons which excite electrons from the sample into free-electron states that can propagate into vacuum; these free electrons are termed photoelectrons. Einstein first described the process in 1905 [1]. He proposed the energy conservation law relating the kinetic energy of these electrons to the light frequency via the quantization of the electromagnetic energy absorbed. Thus did the concept of the photon originate. To bring this idea up to its present level of sophistication, the distribution of photoelectron kinetic energies is measured by an electron energy analyzer or spectrometer, and a "no-loss" photoelectron peak in the resulting spectrum made up of electrons that have not suffered significant inelastic scattering events has a kinetic energy given by

$\mathrm{E}_{\mathrm{k}}=\mathrm{h} v-\mathrm{E}_{\mathrm{b}}-\phi_{\text {spect }}$

where $E_{k}$ is the kinetic energy measured by the photoelectron spectrometer, $E_{b}$ is the binding energy of a given peak (as referenced to the sample Fermi level in work on solid samples), and $\phi_{\text {spect }}$ is the work function of the interior surface of the spectrometer (a constant of the measurement). Although both the outer (valence) and inner (core) electrons can be studied in such spectra, we will here consider only core electrons. The electron binding energies involved can then in first approximation be considered as the one-electron energy levels for the core subshells of the various atoms in the sample, provided we neglect corrections for electron relaxation and other final-state effects 
associated with the hole that is left behind after photoelectron emission. The core-level photoelectron peak intensities can be directly related via well-known photoelectric cross sections to atomic concentration of the sample, thereby providing qualitative and quantitative information as to sample composition. Chemical-state specific data can also be obtained if the atomic core-levels are shifted sufficiently from one state to another via "chemical shifts" or "surface core level shifts". That is, the chemical environment can cause shifts in the binding energies of the core-level electrons, and these shifts in binding energy can be used to determine structural as well as chemical information. A photoelectron spectrum may also contain photon-induced Auger electrons that result from secondary processes involving non-radiative decays of core holes. Auger electrons can be discerned from the "no-loss" photoelectrons by having constant kinetic energies regardless of photon energy. Each photoelectron or Auger peak in a spectrum is also accompanied by an inelastic tail underlying and trailing it. An example of a photoelectron spectrum is given in Fig. 1.1; here the photoelectrons are excited by a nonmonochromatized $\mathrm{Mg} \mathrm{K \alpha}$ laboratory $\mathrm{x}$-ray source at $1253.6 \mathrm{eV}$ and emitted from a clean W[110] surface. Various core-level peaks are visible, including those of W as well as the weaker valence band (V.B.) features near the Fermi energy.

Photoelectron diffraction (PD) involves measuring the angular (or perhaps also the energy) dependence of core photoelectron intensities above a single-crystal sample. The main information derived from the photoelectron diffraction technique is the atomic structure or local atomic geometry around an emitting atom (i.e., the positions of the atoms neighboring it). The first photoelectron diffraction experiments started almost 30 
years ago [2]. In the PD measurement, each photoelectron can be considered to propagate outward from the emitter as a source wave with nearly spherical character $\left(\phi_{0}\right)$; this source wave then scatters from the neighboring atoms around the emitter so as to produce secondary scattered waves $\left(\phi_{\mathrm{j}}\right)$. The interference pattern produced by the superposition of the unscattered source component and the scattered components of the photoelectron wave results in the photoelectron diffraction pattern. The photoelectron intensity in such a pattern as a function of wave vector $\mathbf{k}$ can be written in a simple single scattering picture as:

$I(k) \propto\left|\phi_{o}+\sum_{j} \phi_{j}\right|^{2}$

By measuring the intensity of individual core-level photoelectrons at various directions and/or energies above a single-crystal sample, it is possible to record photoelectron diffraction patterns which have been shown to exhibit strong intensity modulations of up to $\pm 50 \%$. Since we are measuring the intensity of the core-level photoelectrons and each atom's core level energies are well-known and unique we thus know the atomic number and, if chemical shifts are resolvable, also the chemical state or site type, of the emitter. Figs. 1.2(a) and (b) show schematic diagrams of the PD process and a typical experimental geometry for taking scanned-angle experiments, respectively. Some important parameters are as follows: the polar electron emission angle $\theta$ (as measured here from the sample surface), the azimuthal emission angle $\phi$ (measured about the sample normal $\mathbf{n}$ and with respect to a fixed direction lying in the sample surface), the 
angle $\alpha$ between the incident light direction (parallel to the photon wave vector direction q) and the photoelectron spectrometer direction (parallel to the photoelectron wave vector $\mathbf{k})$, the electron-atom scattering factor $f_{j}\left(\theta_{j}\right)$ for a given scattering angle $\theta_{j}$, the photon polarization $\varepsilon$ (shown here for the case of linear polarization), the electron attenuation length $\Lambda_{\mathrm{e}}$ which controls the exponential damping of the no-loss photoelectron signal due to inelastic scattering processes, the inner potential $V_{0}$ which accounts for refraction of the electron wave as it crosses the surface potential barrier and leaves the solid, and the effective analyzer acceptance solid angle $\Omega_{\mathrm{O}}$ over which the actual experiment is averaged. Also important is the damping of diffraction effects due to vibrationally induced atomic disorder, which is usually described via a mean-squared vibrational displacement and a corresponding Debye-Waller factor. From a quantitative analysis of the diffraction pattern based upon these fundamental parameters, one can determine the atomic structure or, if the spin polarization of the photoelectrons is somehow known, also the magnetic order, around a given type of emitting atom [3].

In order to further illustrate the theoretical description of photoelectron diffraction, we will use the simple example in which photoelectrons are being excited from an $\mathrm{s}(l=0)$ core level. Due to the dipole selection rules for the excitation of the photoelectron by the radiation, we know that in general two final state "channels" of $l_{\text {final }}$ $=l_{\text {initial }}+1$ and $l_{\text {initial }}-1$ are possible. Thus, the outgoing wave is for this case $\mathrm{p}$ type $(l=1)$ and it can be shown to contribute a modulation of the source wave of the form $\varepsilon \bullet k$. If we let the detector be positioned at infinity along $\mathbf{k}$, the source and scattered wave components have their limiting spherical forms 
$\phi_{\mathrm{O}} \propto \exp (\mathrm{ikr}) / \mathrm{r}$

$\phi_{j} \propto \exp \left(\mathrm{ik} \mid \mathbf{r}-\mathbf{r}_{\mathbf{j}} \mathbf{j}\right) /\left|\mathbf{r}-\mathbf{r}_{\mathbf{j}}\right|$,

where $\mathbf{r}_{\mathrm{j}}$ is the vector between the emitter and the scatterer. The electron-atom scattering that produces $\phi_{\mathrm{j}}$ can also be described in the simplest approximation by a plane-wave scattering factor

$\mathrm{fj}_{\mathrm{j}}\left(\theta_{\mathrm{j}}\right)=\left|\mathrm{f}_{\mathrm{j}}\left(\theta_{\mathrm{j}}\right)\right| \exp \left[\mathrm{i} \psi_{\mathrm{j}}\left(\theta_{\mathrm{j}}\right)\right]$

where $\psi_{\mathbf{j}}\left(\theta_{\mathbf{j}}\right)$ is the phase shift associated with the scattering angle $\theta_{\mathbf{j}}$. The inelastic scattering is included by an exponential factor $\exp \left[-\mathrm{L}_{\mathrm{O}} / 2 \Lambda_{\mathrm{e}}\right]$ for the source wave and $\exp \left[-L_{j} / 2 \Lambda_{e}\right]$ for the scattered waves, where $L_{o}$ and $L_{j}$ are the distances inside the sample surface each wave component must travel to escape. Vibrational damping is included by the temperature-dependent Debye-Waller factor $\mathrm{W}_{\mathrm{j}}$ which is in turn given by $\exp \left[-2 \mathrm{k}^{2}(1-\right.$ $\left.\left.\cos \theta_{\mathrm{j}}\right) \bar{U}_{j}^{2}(T)\right]$, with $\bar{U}_{j}^{2}(T)$ equal to the temperature-dependent mean-squared vibrational displacement of atom j. We can now rewrite Eq. 1.2 for this special case after some algebra and rearrangement as:

$I(k) \propto\left|(\hat{\varepsilon} \cdot \hat{k}) \exp \left(-L_{o} / 2 \Lambda_{c}\right)+\sum_{j}\left(\frac{\hat{\varepsilon} \cdot \hat{r}_{j}}{r_{j}}\right)\right| f_{j}\left(\theta_{j}\right) \mid W_{j} \exp \left(-L_{j} / 2 \Lambda_{c}\right) \exp \left[i k r_{j}\left(1-\cos \theta_{j}\right)+\left.\psi_{j}\left(\theta_{j}\right)\right|^{2}\right.$ 
For emission from a subshell other than $s$, the above equation will include summations over the different magnetic quantum numbers $m_{\text {initial }}$ and $m_{\text {final }}$ as well as the final-state channels $l_{\text {final }}=l_{\text {initial }}+1$ and $l_{\text {initial }}-1$ allowed under the dipole selection rules; in this case the photoelectron diffraction pattern can be strongly influenced by interchannel interference effects. Other complications that are essential for a fully quantitative description of PD data involve the inclusion of spherical-wave scattering and multiple scattering effects, and methods for carrying out these much more complex calculations are described elsewhere [4].

Several procedures have been used to analyze photoelectron diffraction data so as to derive structural information. For higher-energy photoelectrons, an enhancement in intensity in the forward scattering directions, due to a larger scattering factor amplitude $\left(\left|f_{j}\left(\theta_{j}\right)\right|\right)$ for small scattering angles $\left(\theta_{j}\right.$ approximately equal to zero), often allows one to directly determine bond angles and low index directions from scanned-angle data without resorting to simulations at either the single-scattering or multiple-scattering levels $[3,5]$. Scanned-energy photoelectron diffraction data that is obtained by varying energy at a fixed direction can also be fourier-transformed so as to yield the path-length differences between $\phi_{\mathrm{o}}$ and $\phi_{\mathrm{j}}$ for different atoms $\mathrm{j}$ neighboring a given emitter [3]. A slightly more difficult, but potentially more powerful, technique that is still in the development phase is to carry out a fourier-transform-like manipulation of the photoelectron diffraction pattern as if it were a hologram, which directly leads to three-dimensional atomic positions for different scatterers $[3(\mathrm{c}), 3(\mathrm{~d}), 6]$. But the most rigorous and most accurate way to extract information from a photoelectron diffraction pattern is to compare it to a theoretical 
simulation, and this is the procedure used throughout this thesis. Earlier theoretical models such as that introduced above were more simple and only considered single scattering [3,7-8]. However, in order to quantitatively explain all of the features in photoelectron diffraction patterns, especially for photoelectron diffraction at lower photoelectron kinetic energies and/or for emission along linear chains of atoms for which multiple scattering can be particularly strong, it is necessary to use a more exact treatment of the scattering via a convergent multiple scattering calculation [4]. In analyzing the data obtained in this thesis, three separate multiple scattering codes were used. In early stages of the work, calculations were performed with a program developed by A.P. Kaduwela [4(e)]; this was the first to use a very convenient separable Green's function approach suggested by Rehr and Albers [4(d)] to reduce computation times. The second program was developed by $\mathrm{Y}$. Chen et al. [4(f)], and also makes use of the Rehr-Albers formalism [4(d)]. The third program was developed by F.J. García de Abajo et al. [4(g)] and treats each scattering event in a multiple series exactly. All numerical results reported in this thesis are based on the latter two programs, which were also used to test one another for accuracy.

There are several benefits to using photoelectron spectroscopy and photoelectron diffraction for surface studies. These include:

--Atom (element) specificity, since each element has its own signature set of core electron binding energies, 
--Chemical-state or site specificity, since small changes in chemical state or atomic neighbors can change the binding energy of the electrons enough to be resolved experimentally,

--Variation of light polarization, which can be done with synchrotron radiation sources and allows one to use circular dichroism in photoelectron angular distributions to explore properties of magnetic and non-magnetic samples, --Variation of excitation energy, which is also possible with synchrotron sources, and allows one to increase or decrease the surface sensitivity or atomic cross section for excitation, --Short-range-order sensitivity, due to the short inelastic mean free path and the $1 / \mathrm{r}$ drop-off in the spherical source wave, atomic structure within at most about 20 $\AA$ is probed. Thus, long-range order is not essential, by contrast with other diffraction probes such as LEED.

All of these dimensions of photoelectron spectroscopy and diffraction can thus be fruitfully exploited with higher-brightness third-generation synchrotron radiation, and in particular, we will make use of high energy resolution, faster data acquisition rates, variable polarization, and short-range-order sensitivity.

\section{III. Synchrotron Radiation}

The electromagnetic radiation that is used to excite photoelectrons is clearly a key ingredient of any photoemission measurement. With the availability of so-called thirdgeneration synchrotron sources such as the Advanced Light Source in Berkeley at which 
the experiments for this dissertation were performed, we can obtain extremely high brightness vacuum ultraviolet and soft x-ray radiation that is both tunable in energy and variable in polarization. Such radiation thus opens up entirely new avenues for photoelectron spectroscopy and diffraction studies. For example, one can tune the energy low enough to enhance the surface sensitivity by reducing the inelastic attenuation length of the photoelectrons, vary the polarization so as to measure dichroism effects, and in addition acquire large amounts of high resolution data relatively quickly. This permits doing several new types of experiments: site-resolved photoelectron diffraction from surface and interface atoms, time-resolved measurements of surface reactions with chemical state resolution, and circular dichroism in photoelectron angular distributions from non-magnetic and magnetic systems. Additional possibilities arise for using highresolution photoelectron spectra and photoelectron diffraction data at lower energies to obtain quantitative information on atomic positions and surface chemical reactions. This dissertation will explore all of these dimensions.

Synchrotron radiation is produced by accelerating electrons travelling at relativistic speeds [9] and it is characterized by high brightness and well-defined photon polarization over a wide range of energies (typically ultraviolet to hard x-ray). The thirdgeneration sources of synchrotron radiation that are just coming on line around the world have been designed to produce even brighter light through better collimated and focussed electron beams ("low emittance" beams) and through the use of "insertion devices" which accelerate the electrons many times as they pass along a linear array of permanent magnets. The experiments in this dissertation made use of such low emittance, and were 
performed on bending magnet beamline 9.3.2 of the Advanced Light Source (ALS), one of the first few of such third-generation sources to begin operation. This beamline was designed for high energy resolution spectroscopy with the capability of changing the $\mathrm{x}$-ray polarization from linear to circularly polarized light [10]. The grating monochromator permits varying the photon energy over a very broad energy range from $30 \mathrm{eV}$ to 1500 $\mathrm{eV}$. The combination of this monochromator and a high-resolution, high-throughput electrostatic electron spectrometer in the experimental end station [11] yields overall resolutions of $1: 10^{4}$ in energy. Further details of this facility appear in the next section.

\section{IV. Thesis Outline}

In Chapter 2, we describe the beamline and experimental end station used to collect the data for this thesis, and discuss some of the data analysis procedures utilized. The writer was a key member of the team which designed, assembled, and debugged this system, which first began to take data in October, 1993. This system was built with the idea of exploiting all of the benefits of third-generation synchrotron radiation, and involves several elements that are at the state of the art. Currently it is located at bending magnet beamline 9.3.2 of the Advanced Light Source (ALS), but later this year, it is planned to be moved to undulator beamline 4.0.1 which is equipped with an elliptically polarized undulator (EPU).

In Chapter 3, we examine the well-characterized clean surface of W(110) [12-17], which is used as a reference case to check the accuracy of structure determinations from 
full-solid-angle photoelectron diffraction data via $R$-factor comparisons of experiment with multiple scattering theoretical calculations. The photoelectron energy of $\sim 40 \mathrm{eV}$ used was also lower than in many prior PD studies, providing enhanced surface sensitivity and further challenges to theory. The W(110) surface structure is well known to be nearly bulk terminated; that is, the interlayer spacings near the surface are essentially identical to those in the bulk, with little/no interlayer relaxation [12-17]. The shift in binding energy of the surface atoms relative to the bulk atoms is also relatively large at about $-320 \mathrm{eV}$ and the surface and bulk line widths relatively narrow, allowing easy deconvolution of the two peaks and the separate measurement of both surface and bulk diffraction patterns. In analyzing this data, the influence of various non-structural theoretical input parameters (e.g., scattering phase shifts, electron inelastic attenuation length, and inner potential) was also assessed. A final optimized structure is presented and compared to the results of prior studies [12-17].

In Chapter 4, we next consider a previously undetermined surface structure which arises when one monolayer of an incipient oxide is formed on $\mathrm{W}(110)$. This structure has a long-range periodicity that we find to be $(1 \times 12)$ in symmetry, and we have thus termed it $\mathrm{O} / \mathrm{W}(110)(1 \times 1) \times 12$. This system has been studied before by several different techniques such as low energy electron diffraction (LEED), Auger electron spectroscopy, thermal desorption spectroscopy, work function measurements, and scanning tunneling microscopy (STM) [18-21]. From STM [21], it has been determined that the O sits in the pseudo-threefold hollow site of the W(110) surface, but the height of the O layer above the W layer and the precise lateral position of the oxygen in the threefold site could 
not be determined. In addition, the large unit cell here has prevented using LEED to determine these structural parameters. Thus, we have made use of the site specificity and short-range-order sensitivity of PD to determine both the height of the oxygen and a small shift of the $\mathrm{O}$ atoms from the center of the three-fold hollow site. Once again, the structure determination from the photoelectron diffraction data was via $\mathrm{R}$-factor comparisons of experiment with multiple scattering theoretical calculations.

In Chapter 5, we use chemical-state-resolved core-level photoelectron spectroscopy and diffraction to study the basic kinetics of oxidation of W(110) and the precise adsorption structures involved. By studying the growth and decay with time of various state-resolved peaks in the $\mathrm{W} 4 \mathrm{f}_{7 / 2}$ photoelectron spectra, and comparing our data with the results of a simple Monte Carlo model, we are able to draw some novel conclusions concerning the reaction kinetics involved. We have also measured full-solidangle photoelectron diffraction patterns for two of the oxygen-induced W states, which permit determining the different short-range-order structures involved in the oxidation process for the first time.

In Chapter 6, we take advantage of the variable polarization of the synchrotron radiation from bend magnet radiation and the high-resolution core-level photoelectron spectra obtainable with our end station to study the effects of circularly polarized light on photoelectron diffraction patterns. That is, in connection with core photoelectron emission, it becomes possible to look for circular dichroism effects in which the intensity with excitation by left circular polarization is not equal to the intensity with excitation by 
right circular polarization. Such effects are known to occur for both non-magnetic [2224] and magnetic systems [25]. For the latter, they constitute a novel element-specific way of studying atomic structure and local magnetic order, but the question arises of clearly distinguishing non-magnetic from magnetic circular dichroism effects. To study this relationship, we have performed circular dichroism measurements for the nonmagnetic system of $\mathrm{O} / \mathrm{W}(110)(1 \times 12)$ (the one-monolayer incipient oxide on $\mathrm{W}(110)$ whose atomic structure is determined in Chapter 4). Such non-magnetic circular dichroism in core-level photoelectron angular distributions (CDAD) effects were first observed by Schönhense et al. from CO/Pd(111) [22]. This experiment showed that, even in what are overall non-chiral geometries, circular dichroism may be present in core photoelectron emission. Subsequent to this, Daimon et al. observed similar effects in core emission from $\mathrm{Si}(100)$, and proposed a simple model for predicting the azimuthal peak "rotations" seen between left and right circularly-polarized light [24]. In this chapter, we relate our experimental findings for $\mathrm{O} / \mathrm{W}$ to this prior model and to a more accurate multiple scattering treatment that well predicts all of the changes seen as polarization is varied from left to right circular polarization and to linear polarization.

In Chapter 7, we present some overall conclusions and remarks on interesting future directions for study. 


\section{References:}

[1] A. Einstein, Ann. Physik 17, 132 (1905).

[2] (a) K. Siegbahn, U. Gelius, H. Siebahn, and E. Olsen, Phys. Lett 32A, 221 (1970);

(b) C.S. Fadley and S.A.L. Bergstrom, Phys. Lett 35A, 375 (1971); (c) A. Liebsch, Phys. Rev. Lett. 32, 1203 (1974); (d) S. Kono, C.S. Fadley, N.F.T. Hall, and Z. Hussain, Phys. Rev. Lett. 4, 117 (1978); (e) D.P. Woodruff, D. Norman, B.W. Holland, N.V. Smith, H.H. Farrell, and M.M. Traum, Phys Rev. Lett. 41, 1130 (1978); (f) S.D. Kevan, D.H. Rosenblatt, D Denley, B.C. Lu, and D.A. Shirley, Phys. Rev. Lett. 41, 1565 (1978); (g) S. Kono, S.M. Goldberg, N.F.T. Hall, and C.S. Fadley, Phys. Rev. Lett. 41, 1831 (1978).

[3] (a) C.S. Fadley, Phys. Scripta T17, 39 (1987); (b) C.S. Fadley in "Synchrotron Radiation Research: Advances in Surface and Interface Science", Vol. 1: Techniques, R.Z. Bachrach, Editor (Plenum Press, New York, 1992); (c) C.S. Fadley, Surf. Sci. Repts. 19, 231 (1993); (d) C.S. Fadley et al., J. Electron Spectrosc. 68, 19 (1994); and references therein.

[4] (a) C.H. Li and S.Y. Tong, Phys. Rev. Lett. 42, 901 (1979); (b) J.J. Barton and D.A. Shirley, Phys. Rev. B 32, 1892 (1985); and Phys. Rev. B 32, 1906 (1985); (c) A.P. Kaduwela, G.S. Herman, D.J. Friedman and C.S. Fadley, Phys. Scripta 41, 948 (1990); (d) J.J. Rehr and R.C. Albers, Phys. Rev. B 41, 81139 (1990); (e) A.P. Kaduwela, D.J. Friedman, and C.S. Fadley, J. Electron Spectrosc. 57, 223 (1991); (f) Y. Chen, F.J. García de Abajo, A. Chassé, R.X. Ynzunza, A.P. Kaduwela, M.A. Van Hove, and C.S. Fadley, submitted to Phys. Rev. B; (g) F.J. García de Abajo et al., to be submitted to Phys. Rev. B. 
[5] H.P. Bonzel, Prog. In Surf. Sci. 42, 219 (1993).

[6] J.J. Barton, Phys. Rev. Lett., 61, 1356 (1988); J.J. Barton, J. Electron Spectrosc. and Relat. Phenom 51, 37 (1990).

[7] D.J. Friedman and C.S. Fadley, J. Electron Spectrosc. and Relat. Phenom. 51, 689 (1990).

[8] S.A. Chambers, Adv. In Phys. 40, 357 (1990); S.A. Chambers, Surf. Sci. Repts. 16, 261 (1992).

[9] G. Margaritondo in "Introduction to Synchrotron Radiation" (Oxford University Press, 1988) New York.

[10] Z. Hussain, W.R.A. Huff, S.A. Kellar, E.J. Moler, P.A. Heimann, W. McKinney, H.A. Padmore, C.S. Fadley, and D.A. Shirley, J. Electron Spectosc. and Relat. Phen. 80, 401 (1996); Z. Hussain, W.R.A. Huff, S.A. Kellar, E.J. Moler, P.A. Heimann, W. McKinney, C. Cummings, T. Lauritzen, J.P. McKean, F.J. Palormares, H. Wu, Y. Zheng, A.T. Young, H.A. Padmore, C.S. Fadley, and D.A. Shirley, Rev. Sci. Instrum. 67, 9 (1996).

[11] J. Kortright, M.A. Rice, Z. Hussain, H.A. Padmore, A.T. Young, A. Adamson, W.R.A. Huff, E.J. Moler, S.A. Kellar, R.X. Ynzunza, F.J. Palomares, H. Daimon, E.D. Tober, and C.S. Fadley, Rev. Sci. Instrum. 67, 9 (1996). 
[12] Tran Minh Duc, C. Guillot, Y. Lassailly, J. Lecante, Y. Jugnet, and J.C. Vedrine, Phys. Rev. Lett., 43, 789 (1979).

[13] D.M. Riffe, G.K. Wertheim, and P.H. Citrin, Phys. Rev. Lett., 63, 1976 (1989).

[14] Y. Jugnet, N.S. Prakash, L. Porte, Tran Minh Duc, T.T.A. Nguyen, R. Cinti, H.C. Poon and G. Grenet, Phys. Rev. B 37, 8066 (1988).

[15] M. Arnold, S. Sologub, G. Hupfauer, P. Bayer, W. Frie, L. Hammer, and K., Surf. Rev. Letts., 4, 1291 (1997).

[16] M.A. Van Hove and S.Y. Tong, Surf. Sci. 54, 91 (1976).

[17] J.C. Buchholz, G.C. Wang, and M.G. Lagally, Surf. Sci. 49, 508 (1975).

[18] L.H. Germer and J.W. May, Surf. Sci. 4, 452 (1966).

[19] E. Bauer, Surf. Sci. 7, 351 (1967).

[20] E. Bauer and T. Engel, Surf. Sci. 71, 695 (1978).

[21] K.E. Johnson, R.J. Wilson, and S. Chiang, Phys. Rev. Lett. 71, 1055 (1993).

[22] (a) G. Schönhense, Physica Scripta T 31 (1990) 255; C. Westphal, J. Bansmann, M. Getzlaff, and G. Schönhense, Phys. Rev. Lett. 63 (1989) 151; J. Bansmann, Ch. Ostertag, G. Schönhense, F. Fegel, C. Westphal, M. Getzlaff, F. Schafers, and H. Peterson, Phys. Rev. B 46 (1992) 13496. 
[23] C. Westphal, A.P. Kaduwela, C.S. Fadley, and M.A. Van Hove, Phys. Rev. B 50, 6203 (1994).

[24] H. Daimon, T. Nakatani, S. Imada, S. Suga, Y. Kagoshima, and T. Miyahara, Jpn. J. Appl. Phys. 32, L1480 (1993); H. Daimon, T. Nakatani, S. Imada, and S. Suga, J. Elec. Spec. Rel. Phen. 76, 55 (1995).

[25] A.P. Kaduwela, H. Xiao, S. Thevuthasan, C.S. Fadley, and M.A. Van Hove, Phys. Rev. B 52, 14927 (1995).

[26] L. Baumgarten, C.M. Schneider, H. Peterson, F. Schäfers, and J. Kirschner, Phys. Rev. Lett. 65, 492 (1990). 


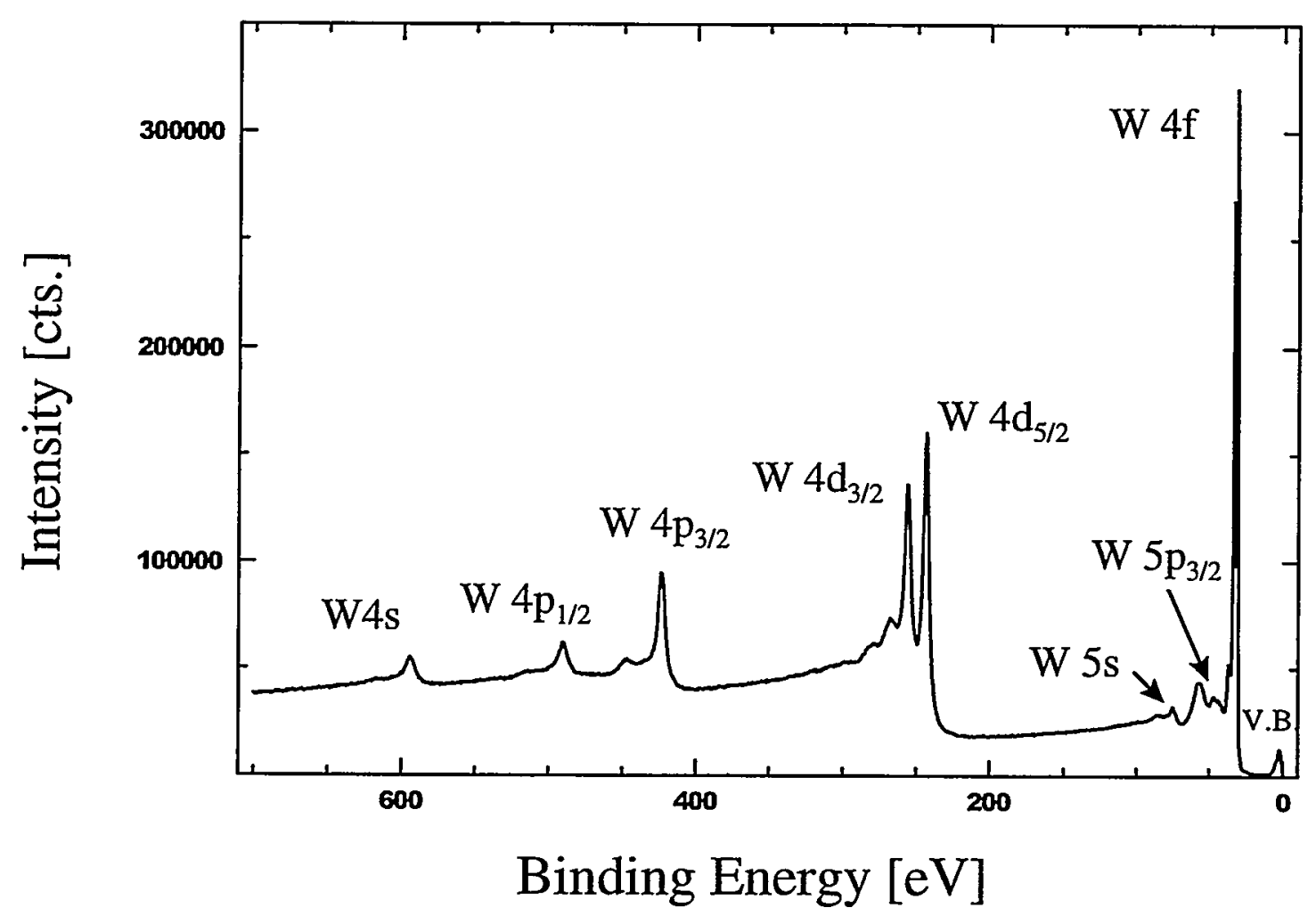

Figure 1.1. Survey spectrum of a clean W(110) surface obtained by exciting with a non-monochromatized $\mathrm{Mg} \mathrm{K} \alpha$ laboratory $\mathrm{x}$-ray source. The core-level photoelectron peaks for $W$ are indicated together with the valence band (V.B.) spectral region. 
a)

\section{Single Scattering Theory}

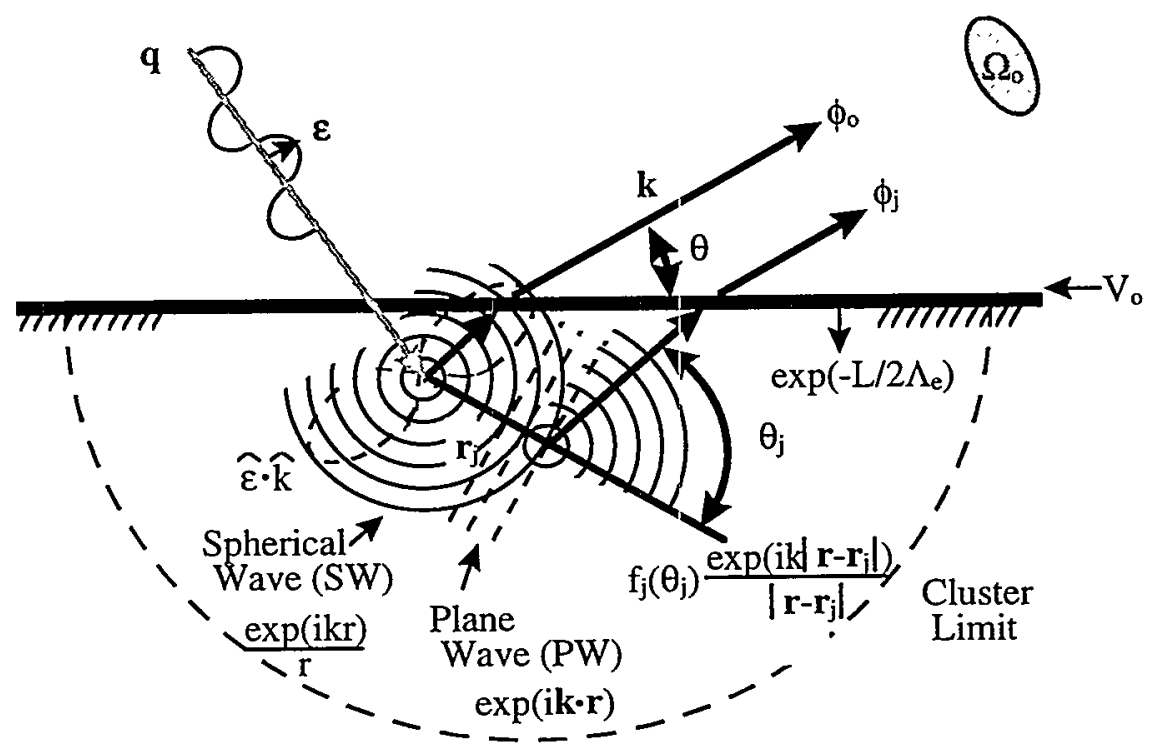

b)

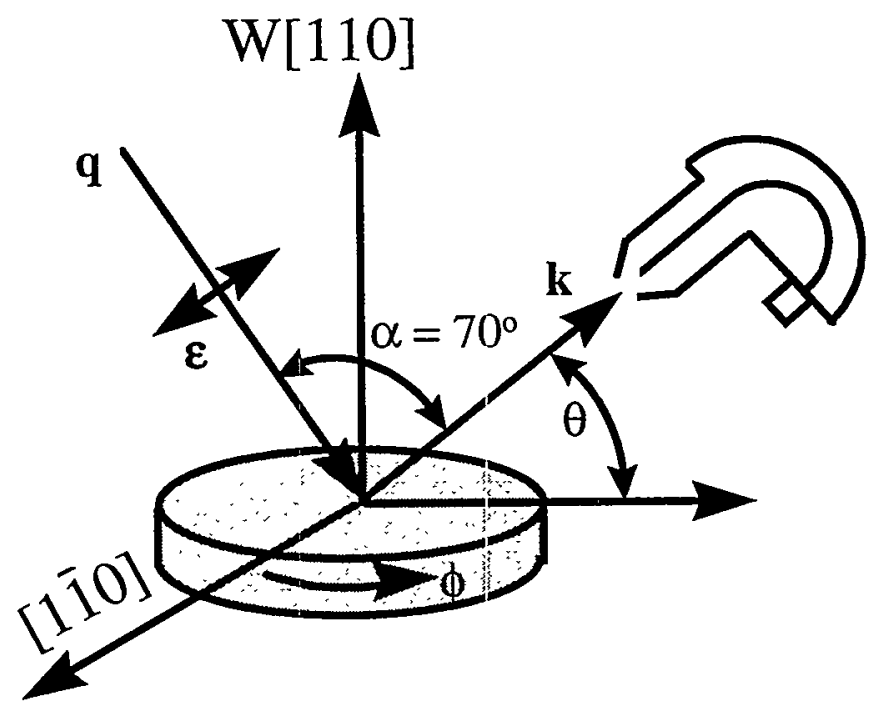

Figure 1.2. a) A schematic representation of the Photoelectron Diffraction (PD) process. Only the case of single scattering is shown for simplicity. b) Illustration of the basic experimental geometry used in PD. 


\section{Chapter 2}

\section{Instrumentation and Data Analysis}

\section{I. Instrumentation Development}

The experiments for this dissertation were performed on beamline 9.3 .2 at the Advanced Light Source (ALS) [1], as illustrated in Fig. 2.1. This beamline was designed for high energy resolution spectroscopy with the capability of changing the light polarization from linear to circular. This beamline has a bend magnet as its radiation source. Bend magnet synchrotron radiation is linearly polarized when collected in the plane of the electron orbit, with the polarization vector lying in the orbit plane. However, a non-zero perpendicular component of polarization can be obtained by collecting the radiation at an angle above or below the orbit plane. This perpendicular component gives rise to elliptically polarized light. The helicity or rotation direction of the polarization vector of the light can be changed by collecting the radiation from either above or below the orbit plane. The amount of circular polarization increases the further one moves from the orbit plane, although the radiation intensity also decreases.

The first set of optical elements in the beamline are horizontal and vertical refocusing mirrors (M1 and M2) in a Kirkpatrick-Baez configuration; these act to deflect the beam and focus it on the entrance slit of the grating monochromator, with a final demagnification of the source by 2:1. Just after its first mirror (M1), beamline 9.3.2 has a narrow water-cooled aperture that can be scanned vertically so as to determine the beam 
centroid (orbit plane) for linearly polarized light; this is an important initial step in calibrating the beamline. A larger aperture as part of the same mechanism can also be moved vertically so as to select light from some selected distance above and below the beam centroid, thus obtaining circularly polarized light. In this way, circular polarization of $80-85 \%$ was achieved with an intensity loss of only a factor of $1 / 3$ to $1 / 4$ [1]. With the 2:1 demagnification, the source at the entrance slit of the monochromator has a vertical size of approximately $50 \mu \mathrm{m}$ and a much larger horizontal size of approximately $6 \mathrm{~mm}$. A final bendable refocusing mirror (M3) after the monochromator focuses the light back onto the sample, yielding typically a spot of $0.5 \mathrm{~mm}$ in vertical size and $1.0 \mathrm{~mm}$ in horizontal size.

The Rowland circle spherical grating Monochromator (SGM) has three interchangeable gratings with 100 lines $/ \mathrm{mm}, 600$ lines $/ \mathrm{mm}$, and $1200 \mathrm{lines} / \mathrm{mm}$ so as to access photon energies from $30 \mathrm{eV}$ to $1500 \mathrm{eV}$. During this thesis research the third highenergy grating with 1200 lines/mm was not operating properly, so the effective range of utilization with the other two gratings was $30 \mathrm{eV}$ to $800 \mathrm{eV}$. This monochromator also has movable entrance and exit slits so that the Rowland-circle condition can be met for all energies; these slits are under computer control, as is the variation of the monochromator energy. The ultimate energy resolution of the beamline is $1: 10^{4}$ of the photon energy, with actual measurements on $\mathrm{N}_{2}$ photoionization showing resolving powers of at least 8,000 . The entrance slit for the majority of experiments is set to a vertical width of $10-50$ $\mu \mathrm{m}$. Because these values are comparable to the vertical size of the focussed incoming beam, any misalignment or movement of the incoming beam can cause incomplete filling 
of the source on the entrance slit, thus leading to changes in the transmitted flux. To counteract this flux stability problem (which was rather serious in our first experiments with the beamline), the vertical mirror (M2) and the entrance slit are coupled in a feedback arrangement which maintains the synchrotron light centered on the slits. This keeps small motions in the electron storage ring source and instabilities in the mirrors from affecting the photon flux and energy. As a final monitor of the actual photon flux for normalizing the data so as to derive what is often termed an $I_{0}$ correction, there is a gold grid in the path of the beam just before the experimental chamber whose photoemitted drain current is assumed to be proportional to this flux.

Part of this thesis work involved assisting with the commissioning of this beamline, which was the first at the ALS to permit variable-polarization studies. Additional details on the design and operation of it appear elsewhere [1].

At the end of the beamline, there is a rotating platform which allows switching between two experimental endstations (the system used in this work, and other general user endstations) without breaking vacuum, as shown in Fig. 2.2. This platform rotates over $60^{\circ}$ via a bellows connection to the rest of the beamline. Also shown in this figure is a horizontal deflection mirror that can be inserted in the beam path to permit doing soft $\mathrm{x}$-ray interferometry, but this was not relevant to the present research.

At the end of this beamline is the Advanced Photoelectron Spectrometer/Diffractometer (APSD), whose design and construction constituted a major 
endeavor during the initial years of this thesis research. Various aspects of this system are shown in Figures 2.3-2.10. It consists of a rotateable main analysis chamber (Fig. 2.7) to which is attached the electron spectrometer (Fig. 2.3), an isolateable preparation chamber mounted on top of the main chamber (Figs. 2.5 and 2.6), a specially-designed two-axis variable-temperature sample goniometer which can be translated in three dimensions (Figs. 2.8 and 2.9), and various other sample cleaning, preparation, and characterization facilities that are mounted on its ports. The pumping system is also shown in Fig. 2.10.

The electron energy spectrometer (Scienta SES200), as shown in detail in Fig. 2.3, consists of a hemispherical electrostatic analyzer with a Herzog-plate field termination and an accelerating-retarding multi-element electrostatic lens at its entrance [2]. The mean radius of the hemispheres is $200 \mathrm{~mm}$. This lens serves to both retard the electron energy from its initial value $E_{k}$ to some lower pass energy $E_{\text {pass }}$ at which the deflection and analysis is actually performed and to magnify the source size by $5 x$. Several entrance apertures to the hemispherical deflection region can also be selected, with each one of these being consistent in both source size and accepted solid angle with a certain resolution as judged by $\Delta \mathrm{E} / \mathrm{E}_{\text {pass }}$. With the narrowest aperture in both size and solid angle, the analyzer resolution is $\Delta E / E_{\text {pass }}=0.1 \%$. Thus, with a $2 \mathrm{eV}$ pass energy, we have obtained in initial tests of the system a resolution of $2.9 \mathrm{meV}$ that is very close to $0.1 \%$. We also verified in initial tests that the narrowest aperture yielded $\Delta \mathrm{E} / \mathrm{E}_{\text {pass }}=$ $0.1 \%$ (or actually somewhat less than this) in a series of measurements with high-energy $\mathrm{Al} \mathrm{K} \alpha$ x-ray excitation of $\mathrm{Ag} 3 \mathrm{~d}$ levels and a variety of pass energies. Retarding by a 
typical value of 10 from $E_{k}$ to $E_{p a s s}=E_{k} / 10$, then yields an overall resolution of $\Delta E / E_{k}=$ $0.01 \%$ or $1: 10^{4}$. The lens magnification also means that, for a given aperture size, the area of the sample seen by the analyzer is only $1 / 5$ of this. The angular acceptance of the analyzer is nominally $\pm 5^{\circ}$ and can be reduced to $\pm 1.5^{\circ}$ by inserting a specially-designed double-pinhole collimator in front of the lens. After deflection over $180^{\circ}$ of arc in the hemispherical electrodes, electrons of a given energy are brought to a focus at various radial positions and at various distances above and below the symmetry plane of the hemispheres. By finally detecting electrons over this two-dimensional area, in which the radial dimension is energy, the overall spectrum accumulation rates are much improved. At the time the data for this dissertation were taken, the final electron detector consisted of two multichannel plates to amplify the incident photoelectrons, a phosphorescent screen to turn each amplified electron signal into a light pulse, and a CCD camera outside of vacuum to read these light pulses and encode them in two dimension. In its initial configuration, this system could handle a maximum count rate of $100 \mathrm{kHz}$ over the 1 " diameter of the detector. Currently the detector can run in a so-called "grey-scale" mode that increases the count rate to $600-700 \mathrm{kHz}$. This analyzer thus combines the attributes of high energy resolution with high throughput. The maximum energy resolution of $1: 10^{4}$ also matches that of the beamline monochromator, which keeps either one from becoming a limiting factor for high resolution experiments. This analyzer also has extremely stable power supplies, which is important for attaining the highest possible energy resolution and for obtaining correctly calibrated electron energies. An additional special feature of the APSD is that the detector can be changed in-situ by rotating between two detectors mounted with linear drives to a carousel which rotates on a 
differentially pumped rotary seal, as shown in Fig. 2.4. This last feature was not used in the present research, but will in the future accommodate a much higher speed multichannel detector operating in the $\mathrm{GHz}$ range and a single-channel spin detector based on Mott scattering.

The main analysis chamber, as shown in Figs. $2.5-2.7$, is rotateable by $60^{\circ}$ in the plane of the electron storage ring. This rotation is illustrated in Fig. 2.7 [3]. This inplane rotation allowed us to keep the photon-sample angle fixed while still being able to measure intensities over a significant portion of the $2 \pi$ solid angle above the sample surface, as will be essential for the work to be discussed in Chapter 5. This rotation is achieved by using two-stage differentially pumped seals at the top and bottom of the chamber and a large diameter bellows connecting the chamber to the beamline. The graphite-impregnated Teflon seals (Balseal) were incorporated into these custom-made seals by APX Scientific and designed to handle the unusual and large lateral vacuum loading force $(1700 \mathrm{lbs}$.) due to the large diameter bellows. The bellows is $19 "$ long and $13.75^{\prime \prime}$ in diameter which allows direct line of site for the synchrotron light between the last refocusing mirror and the sample over the required $\sim 60^{\circ}$ of rotation. When synchrotron light is not available, there is also a dual-anode $\mathrm{Al} \mathrm{K} \alpha$ and $\mathrm{Mg} \mathrm{K} \alpha$ lab X-ray source (Perkin Elmer Model 04-548) installed. There is also a low energy electron flood gun in the main chamber for neutralizing sample charge buildup on insulating samples.

The preparation chamber, also shown in Figs. 2.5 and 2.6, is separated from the analysis chamber by a viton-sealed gate valve. Closing this prevents any sample 
preparation procedure from contaminating the main analysis chamber. The preparation chamber has two water-cooled Knudsen cells for metal deposition, along with an Inficon model XTC quartz crystal oscillator that is used to monitor film thickness. There is also a large gap electromagnet that is used for magnetizing samples, an ion sputtering gun, and a reverse view optics for low energy electron diffraction (Princeton Research Model 118).

The sample manipulator, as shown in Fig. 2.8, has three degrees of linear motion: a long $\mathrm{z}$ motion allowing it to move vertically between the two chambers, and two more limited $\mathrm{x}$ and $\mathrm{y}$ motions for positioning the sample horizontally in the chamber. The twoaxis goniometer mounted at the end of the manipulator, driven by two high-precision rotary feedthroughs shown in Fig. 2.8, is shown in more detail in Fig. 2.9. This goniometer has a high angular accuracy $\left( \pm 0.3^{\circ}\right)$ in its two degrees of angular motion: polar $(\theta)$ and azimuthal $(\phi)$. There are three different types of sample heating available: a non-inductive resistive coil, electron bombardment on the back of the sample, and direct current heating by passage of current through the sample. In the present work, only electron bombardment heating of tungsten was used. Liquid nitrogen cooling is also possible via a copper braid and detachable spring-actuated copper cold finger. This combination allows samples to have a temperature range between $200 \mathrm{~K}$ and $2300 \mathrm{~K}$, although no cooling was necessary for the work reported here. There is also an in-vacuo sample carousel that permits loading up to eight samples initially and switching them off and on the goniometer without breaking vacuum by using a wobble stick (as shown in Fig. 2.9(b)). Finally, three reference samples are permanently mounted below the primary sample position to permit calibration and alignment of the system; these normally 
consisted of a large diameter phosphor to locate the light beam, a small gold dot to determine where the spectrometer is actually looking, and a large diameter gold sample for resolution and intensity calibration.

Because the APSD system is designed to work at ultrahigh vacuum conditions in the low $10^{-11}$ to low $10^{-10}$ range, its pumping and valving system is a crucial subsystem of the overall facility. In Fig. 2.10 all of the main pumps and valves, and their interconnections are shown. Primary UHV pumping in the chambers is by ion pumps (IPs), assisted by water cooled titanium sublimation pumps (TSPs). Roughing of the chambers down from atmosphere is via turbomolecular pumps (Turbos), which are in turn backed by diaphragm pumps. Differential pumping of the three rotating seals (two for the main analysis chamber and one for the detector carousel) is via turbomolecular pump or a scroll pump for the outer seals and small dedicated ion pumps for the inner seals.

This experimental system, while very comprehensive and unique in several aspects for photoelectron experiments, is still under development. Several more projects are scheduled to enhance capabilities:

--installation of a loadlock system in the preparation chamber, which will permit inserting and removing samples in a very short time of a few hours, instead of the present requirement to vent the preparation chamber, load up to 8 samples, and then bake the chamber and goniometer fully, which takes $2-3$ days, 
--replacement of the commercial software for controlling the analyzer with a much more flexible and user-friendly control system that will permit scanning not only sample angles but also photon energy, and doing on-line data analysis of photoelectron diffraction patterns,

--replacement of the CCD-based detector on the analyzer with a much higher speed multichannel detector, which will have an overall count rate capability in the $\mathrm{GHz}$ range,

--addition of a micro-Mott spin detector in the second position of the detector carousel, which will enhance studies of magnetic materials,

--design and construction of a new sample goniometer with higher angular resolution and the ability to go to lower temperatures in the $10-30 \mathrm{~K}$ range with liquid helium cooling, --addition of a surface magnetooptical Kerr effect system to the preparation chamber, which will enhance studies of magnetic materials,

--additional usage of the APSD endstation on a soon-to-be-commissioned elliptically polarized undulator beamline 4.0.1 at the ALS, which should result in 10-50 times higher photoelectron intensities and greater flexibility in varying radiation polarization, --addition of a scanning tunneling microscope (STM) to the preparation chamber, which will permit doing pre- and post- experiment analysis of surface morphologies. 


\section{II. Data Analysis}

\section{Peak intensity determinations:}

Peak intensities were determined by fitting each spectrum with Voigt functions (a convolution of Lorentzian and Gaussian shapes) riding on a Shirley-type inelastic photoelectron background [4]. An example of a fitted spectrum is shown in Fig. 2.11. The fitting was done with a commercial fitting package provided with the data acquisition software for the Scienta SES200 spectrometer. From a physical point of view, the inelastic background is comprised of inelastically scattered photoelectrons due to a given photoelectron peak itself, riding on the inelastically scattered electrons from all electrons at higher kinetic energies. The former contribution thus varies over the spectrum, while the latter can usually be assumed to be a constant underlying the entire spectrum. In order to subtract the former type of background, the software makes use of the Shirley-type algorithm [4]. This procedure first selects two points in the spectrum, one on the highkinetic-energy side of the spectrum and one on the low-kinetic-energy side of the spectrum. The intensity of this background at any point in between is then proportional to the sum of the intensity of the photoelectron peak minus the background of the entire spectrum to higher kinetic energies, with the condition that the background contains the two reference points. An initial assumption about the form of the inelastic background is then iterated to self-consistency, with this process converging in a few seconds in the software utilized. 
There are two major contributions to the photoelectron peak shape: the photoelectron line shape associated with processes intrinsic to the sample and the instrumental broadening. The contribution to the peak from instrumental broadening is mostly of a Gaussian nature and given by the equation,

$$
G(E)=H \exp \left[\frac{-4 \ln 2\left(E-E_{0}\right)^{2}}{(F W H M)^{2}}\right]
$$

where $G$ is the Gaussian peak shape, $E$ is the kinetic energy, $H$ is the height of the peak, $E_{0}$ is the center kinetic energy of the peak, and FWHM is the full-width at half-maximum of the peak. The contribution from the lifetime associated with the core hole left behind after photoelectron excitation is Lorentzian in nature and is given by the equation,

$$
L(E)=H\left[1+\frac{4\left(E-E_{0}\right)^{2}}{(F W H M)^{2}}\right]^{-1}
$$

where $\mathrm{L}$ is the Lorentzian peak shape. The convolution of the Gaussian and Lorentzian peak shapes, known as a Voigt function, is thus needed to accurately describe a peak shape that includes both instrumental broadening and the contribution from the lifetime. In general, other final-state effects (many-electron excitations, vibrational excitations) may cause additional asymmetries and deviations from this peak shape, but it was found possible in any case to describe our spectra very accurately with Voigt function fitting. In 
order to save time in the fitting procedure, the software handles this convolution by using the approximation of a linear combination of Gaussian and Lorentzian components with the same energy and width [5]. The resulting equation for the Voigt function is

$V(E)=m G(E)+(1-m) L(E)$

where $\mathrm{V}$ is the Voigt peak shape, and $\mathrm{m}$ is the mixing parameter. This peak is added to any other similar peaks that may be fitted simultaneously and the background. The program makes use of a non-linear peak fitting procedure, optimizing the parameters iteratively using $X^{2}$ as a merit function. Initial trial values for the parameters must be supplied to the program and the program iteratively improves these parameters.

When fitting the many spectra involved in large-solid-angle photoelectron diffraction data, each azimuthal scan at a given polar angle was fitted separately. The first angle in the scan was fitted with all the parameters floating. When a reasonable result was obtained, all the parameters for fitting the rest of the scan are held fixed except for the peak heights (and thus also areas). Each azimuthal angle in a scan was fitted consecutively, with the height of the previous angle in the azimuthal scan as the initial height parameter. As a second step, each azimuthal scan was fitted again, this time with all the parameters allowed to float and the initial values chosen to be the previous fitted values for the same angle. Thus, the FWHMs, the peak positions, the Gaussian/Lorentzian mixes, and the intensity were slightly different for each angle at this step of the analysis. As a third step, the FWHMs, the peak positions, and the mixing 
parameters were fixed to the mean values determined from the previous fitting, with only the intensity parameters $\mathrm{H}$ allowed to vary. The last two steps may be repeated for several of the parameters, depending on how well the fit agrees with the experimental data. The final fit will always have all of the parameters for the azimuthal scan fixed except for the height since we can assume from the physics of the measurement that the parameters FWHM, position, and mix should not depend on the azimuthal angle (or at most depend on it very weakly). Fitting the data in this way avoids spurious changes in peak relative intensities due to slight changes in these three parameters that may lead to a slightly better fit but are not physically reasonable adjustments.

\section{Normalization of diffraction patterns:}

Correction for storage ring current decay: After the experimental data has been fitted, the individual experimental peak areas are collected as a function of theta $(\theta)$ and phi $(\phi)$, i.e. $I_{\text {expt }}(\theta, \phi)$. Corrections are then made for time-dependent beam decay in the storage ring. With typical times between fills of the storage ring of 4 hours, a decay in current over this time to about $50 \%$ of its initial value, and typical data set acquisition times of at least a few hours, this correction is crucial. As noted above, the secondary electron current from a gold grid located directly before the sample is used to monitor the photon flux incident on the sample. The drain current of the gold grid is assumed to be proportional to the amount of flux on the sample. Therefore, the corrected intensity is finally given as $\mathrm{I}(\theta, \phi)=\mathrm{I}_{\text {expt }}(\theta, \phi) / \mathrm{I}_{\mathrm{GG}}$, where $\mathrm{I}$ is the adjusted intensity and $\mathrm{I}_{\mathrm{GG}}$ is the drain current read from the gold grid. 
Correction for geometrical intensity modulation: Due to well-known geometrical effects, the intensities of photoelectrons emitted at lower takeoff angles with respect to the surface usually have a lower intensity. Due to this drop-off in intensity, it is difficult to observe in a straight plot of $\mathrm{I}(\theta, \phi)$ the oscillations in the diffraction pattern for small $\theta$ values, and it is thus not optimal to have the intensity plotted just as it is collected. To correct for these geometrical effects, we desire to normalize the data with a smooth function $\left(I_{0}\right)$ that is intended to represent all angular-dependent effects outside of photoelectron diffraction. Provided such a function can be found, he data are then normalized in a standard way into a chi function as

$\chi(\theta, \phi)=\left(I(\theta, \phi)-\mathrm{I}_{0}(\theta)\right) / \mathrm{I}_{0}(\theta)$.

The smooth function $\left(\mathrm{I}_{0}\right)$ was determined in two steps. First, the data was averaged in $\phi$ for each polar angle $(\theta)$ giving us $I_{\text {ave }}(\theta)$. Next, the average intensities were fitted by a low-order spline function that is not required to go through the points, such that the spline does not show any significant oscillations on the same scale as the diffraction modulations. The result of such a spline fit to $W 4 f_{7 / 2}$ data from clean $W(110)$ is the $I_{0}(\theta)$ as shown in Fig. 2.12.

\section{Surface quantitative analyses from photoelectron intensities:}

Core photoelectron peak intensities can be used to determine surface chemical 
compositions, contamination levels, and overlayer coverages and thicknesses [6,7]. Or, if the sample is sufficiently well characterized, such an analysis can be inverted to determine the inelastic electron attenuation length [8]. Standard equations exist for using experimental intensities to determine these quantities, provided that certain other parameters can be derived from either experiment or theory. For example, the experimental variation of the analyzer transmission with pass energy [9] and experimental or theoretical relative photoelectric cross sections [10] are essential in this respect.

In this thesis, such quantitative analysis has been done to verify the surface cleanliness and compositional stability before, during, and after a series of measurements. The specific results of these analyses are given in the following chapters, but we here use one case as an illustrative example of how such an analysis proceeds. This is the determination of the oxygen coverage in monolayers (MLs) of the (1x1)-(1x12) incipient oxide overlayer on W(110). To do this, we used the standard result applicable to a semiinfinite substrate with uniform weakly-attenuating overlayer [6,7], for which the fractional coverage $\Theta$ in $\mathrm{ML}$ can be expressed as:

$$
\Theta=\frac{N_{j}(\theta)}{N_{k}(\theta)} \frac{A_{o}\left(E_{k}, \theta\right) \Omega_{o}\left(E_{k}\right) D_{o}\left(E_{k}\right)\left(d \sigma_{k} / d \Omega\right)}{A_{o}\left(E_{j}, \theta\right) \Omega_{o}\left(E_{j}\right) D_{o}\left(E_{j}\right)\left(d \sigma_{j} / d \Omega\right)} \frac{\Lambda_{c}\left(E_{k}\right) \sin \theta}{d}
$$

where $E_{k}$ is the kinetic energy of a peak from the substrate (here W(110)), $E_{j}$ is the kinetic energy of a peak from the overlayer (here oxygen), $N_{k}(\theta)$ is the intensity of a peak from the substrate (W $4 f$ or one of its state-resolved components), $N_{j}(\theta)$ is the intensity of 
a peak from the overlayer $(\mathrm{O} 1 \mathrm{~s}), \Omega_{\mathrm{o}}$ is the energy-dependent effective solid angle seen by the spectrometer, $A_{0}$ is the energy-dependent effective specimen area seen by the spectrometer, $D_{o}$ is the energy-dependent efficiency of the detector, $\left(d \sigma_{k} / d \Omega\right)$ is the differential photoelectric cross section of the substrate peak (which depends on photon energy), $\left(\mathrm{d} \sigma_{\mathrm{j}} / \mathrm{d} \Omega\right.$ ) is the differential photoelectric cross section of the overlayer peak (also dependent on photon energy), $d$ is the atomic interplaner distance in the substrate ( $2.23 \AA$ for $\mathrm{W}(110)$ ), and $\Lambda_{e}$ is the inelastic attenuation length in the substrate. In what follows, we will consider analyses done with two different experimental systems: a VG ESCALAB5 laboratory system with an unpolarized x-ray source and the APSD at the ALS.

The effective specimen area $A_{0}$ can be assumed to identical for the substrate and overlayer peaks and can be canceled out in the ratio. However, the kinetic energy dependent instrumental transmission function $T\left(E_{\text {kin }}\right)$, which is represented by the combined product $\Omega_{0}\left(E_{k i n}\right) D_{o}\left(E_{k i n}\right)$, has to be determined for the two different photoelectron peaks involved. It is also well known that $T\left(\mathrm{E}_{\mathrm{kin}}\right)$ can to good accuracy be assumed to be simply proportional to $\left(\mathrm{E}_{\mathrm{kin}}\right)^{-\mathrm{q}}[7]$. The value of $\mathrm{q}$ was determined to be 0.70 for the VG ESCALAB5 system used for this analysis, and it is much smaller at $q=$ 0.15 for the Scienta SES200. That is, the Scienta has the advantageous feature of a very weak dependence in transmission on electron kinetic energy, for a given pass energy. The differential photoelectric cross sections are calculated in the standard manner [9]: 
Unpolarized radiation:

$$
\frac{\mathrm{d} \sigma(\alpha)}{\mathrm{d} \Omega}=\frac{\sigma}{4 \pi}\left[1+\beta / 2\left(3 / 2 \sin ^{2} \alpha-1\right)\right]
$$

Polarized radiation:

$$
\frac{\mathrm{d} \sigma(\gamma)}{\mathrm{d} \Omega}=\frac{\sigma}{4 \pi}\left[1+\beta\left(3 / 2 \cos ^{2} \gamma-1 / 2\right)\right]
$$

where $\sigma$ is the total cross-section of a given photoelectron peak, $\beta$ is the asymmetry parameter for the photoelectron peak, $\alpha$ is the angle between the incident light and the emitted photoelectron ( $\alpha=48^{\circ}$, for the VG ESCALAB5 used here), and $\gamma$ is the angle between the light polarization direction and the emission direction (variable for the APSD, since the chamber is rotateable). In the ESCALAB experimental system we used to determine the oxygen coverage of the $(1 \times 1) \times 12$ structure, we had an unpolarized source. Tabulations of $\sigma$ and $\beta$ appear in the literature [10]. An attenuation length for W 4f photoelectrons in W at a kinetic energy of $1455 \mathrm{eV}$ of $\Lambda_{\mathrm{e}}=18.1 \AA$ was determined by extrapolation of a similar quantitative calculation with a clean tungsten surface, as described elsewhere [8]. $\Lambda_{\mathrm{e}}$ was estimated from an experimental value at lower energy [8], as extrapolated to higher energy using the relation $\Lambda_{e}=\mathrm{CE}^{1 / 2}$, where $\mathrm{C}$ is an empirical constant and $\mathrm{E}$ is the electron kinetic energy. This formula was in turn derived 
by Seah and Dench using experimental attenuation lengths for several solid elements [11]. The parameter $\mathrm{C}$ was determined from an experimental determination for the clean $W(110)$ surface of $3.0 \AA$ at a kinetic energy $E$ of $4.0 \mathrm{eV}[8]$.

In order to minimize possible errors in the $\mathrm{O}$ and $\mathrm{W}$ intensities due to diffraction effects, a full-solid-angle photoelectron diffraction pattern was taken for both the $O$ is and state-resolved bulk W $4 \mathrm{f}$ photoelectron peaks as integrated over both the bulk and incipient-oxide features seen in Fig. 2.11, and then the data was averaged in $\phi$ for each polar angle $(\theta)$. Thus, $\mathrm{N}_{\mathrm{k}}(\theta)\left(\mathrm{N}_{\mathrm{j}}(\theta)\right)$ is the average $\mathrm{W}$ 4f (O 1s) intensity for the polar angle $\theta$. Then to find the final oxygen overlayer coverage, an average was taken over the coverage from each polar angle, as shown in Fig. 2.13, to yield a final value of $1.04 \pm$ $0.07 \mathrm{ML}$ that is in excellent agreement with the expected 1.00 ML for this saturated overlayer. 


\section{References:}

[1] (a) Z. Hussain, W. R. A. Huff, S. A. Keller, E. J. Moller, P. A. Heimann, W. McKinney, H. A. Padmore, C. S. Fadley, and D. A. Shirley, J. Elec. Spec. and Rel. Phen. 80, 401 (1996) and abstract by E.J. Moler et al. in the Advanced Light Source Compendium of Users Abstracts and Technical Reports, 1993-1996, Report LBNL-39981, UC-411, p. 336.

[2] M. Martensson, P. Baltzer, P.A. Bruhwiler, J.-O. Forsell, A. Nilsson, A. Stenborg, and B. Wannberg, , J. Elec. Spectrosc. and Relat. Phenom. 70, 117 (1994).

[3] C.S. Fadley, M.A. Van Hove, Z. Hussain, and A.P. Kaduwela, J. Elec. Spec. and Rel. Phen. 75, 273 (1995) and abstract by R.X. Ynzunza et al. in the Advanced Light Source Compendium of Users Abstracts and Technical Reports, 1993-1996, Report LBNL-39981, UC-411, p. 332.

[4] D.A. Shirley, Phys. Rev. B55, 4709 (1972).

[5] G.K. Wertheim, M.A. Butler, K.W. West, and D.N.E. Buchanan, Rev. Sci. Instrum., 45, 1369 (1974).

[6] C.S. Fadley, Prog. In Surf. Sci., 16, 275 (1984).

[7] C.S. Fadley in "Electron Spectroscopy, Theory, Techniques and Applications", Vol. 2, C.R. Brundle and A.D. Baker (Eds.), (Pergamon Press, 1978).

[8] R.X. Ynzunza, Ph.D. thesis, University of California-Davis (1998), Chapter 3. 
[9] J. Osterwalder, M. Sagurton, P.J. Odrers, C.S. Fadley, B.D. Hermsmeier, and D.J. Friedman, J. Electron Spectrosc. Relat. Phenom., 48, 55 (1989)

[10] S.M. Goldberg, C.S. Fadley, and S. Kono, J. Electron. Spectrosc. 21, 285 (1981); J.J. Yeh and I. Lindau, Atomic Data and Nuclear Data Tables 32, 1 (1985).

[11] M.P. Sean and W.A. Dench, Surf. \& Interf. Anal. 1, 2 (1979). 


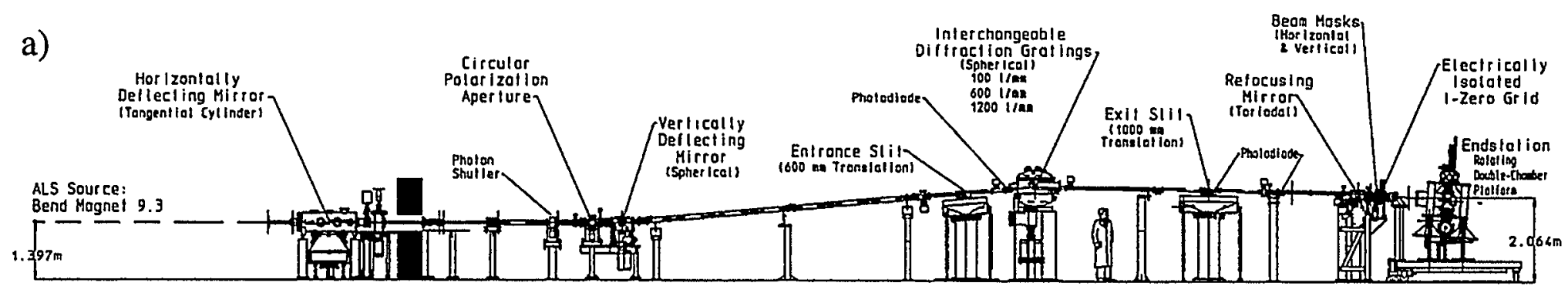

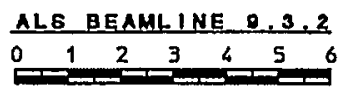

b)

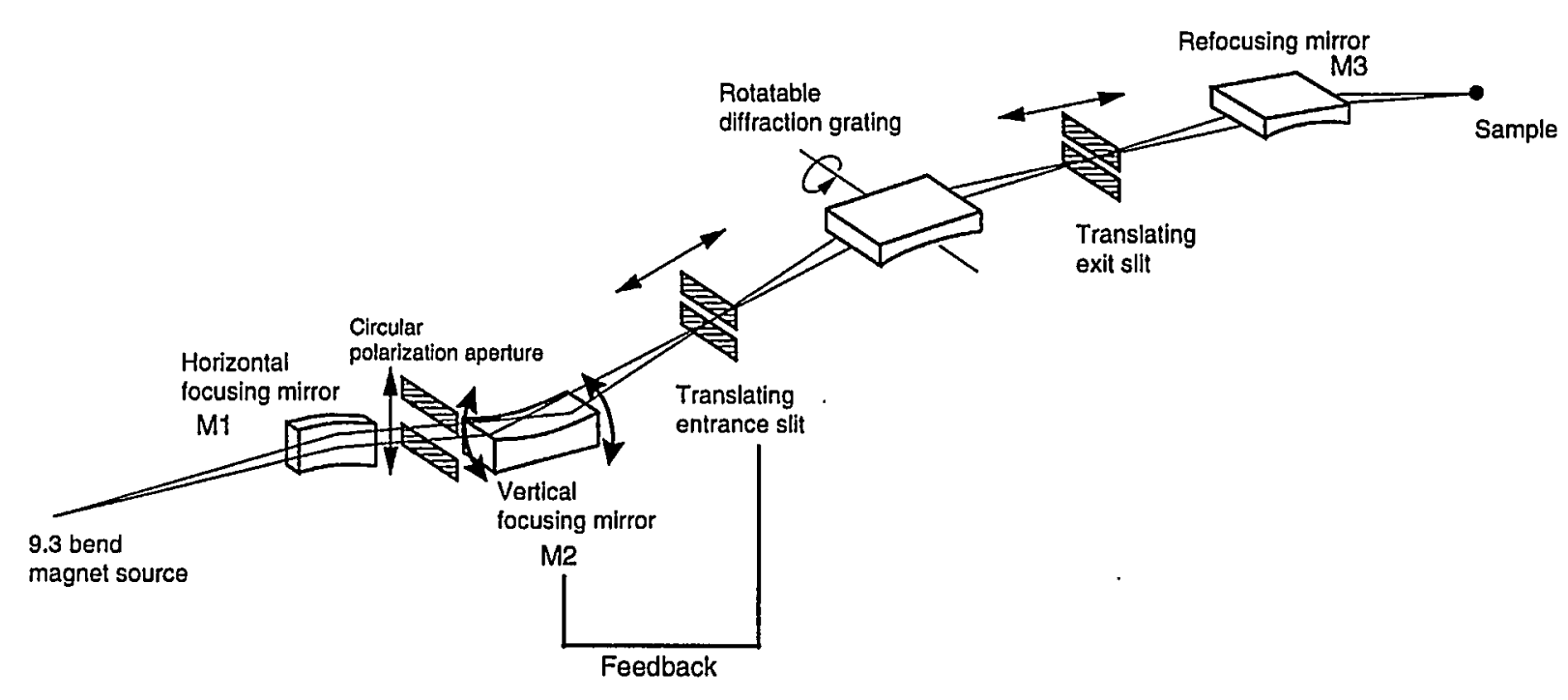

Figure 2.1. a) Scale drawing and b) schematic of beamline 9.3.2 at the Advanced Light Source. 


\section{BEAMLINE O.3.2}

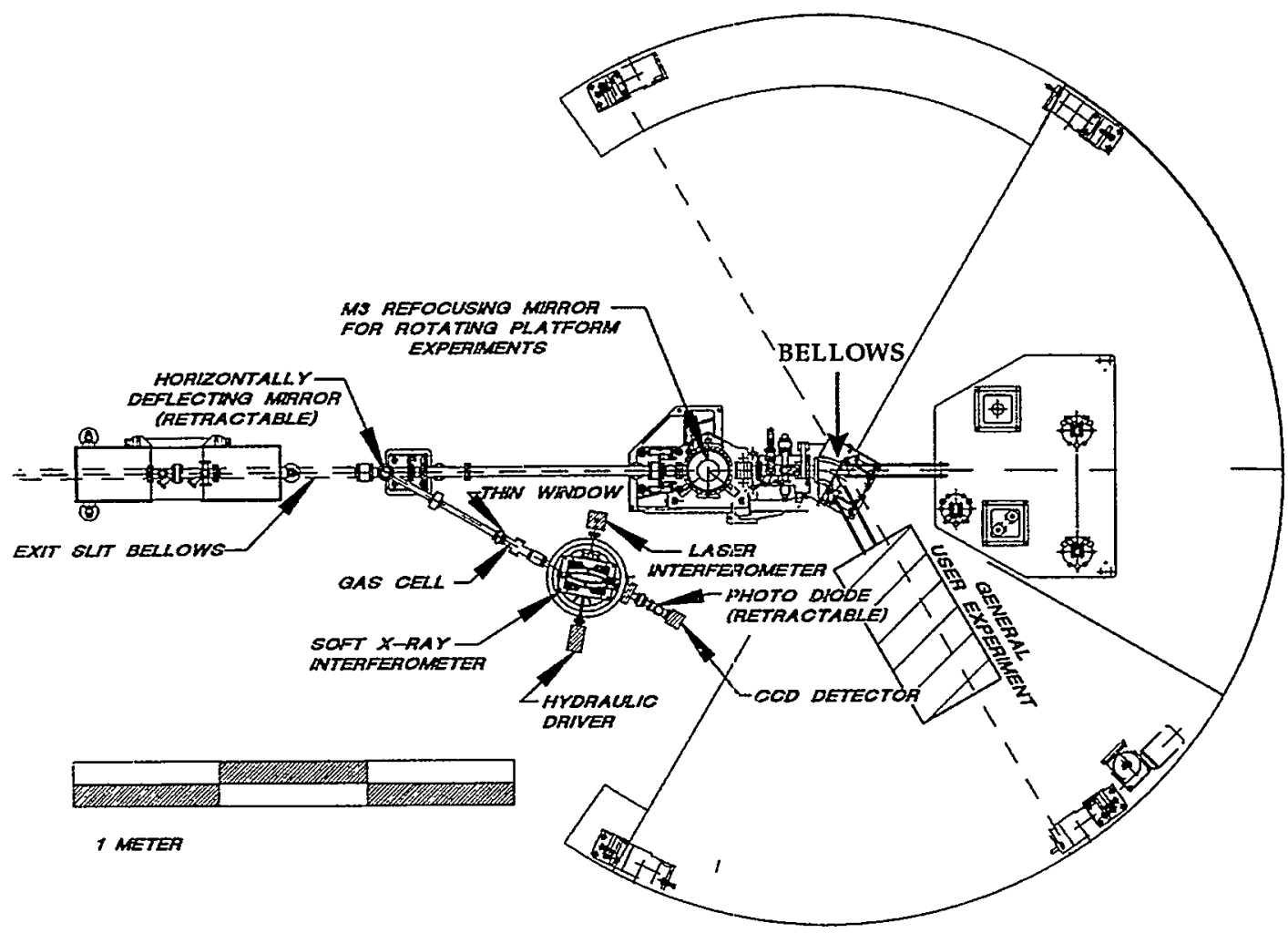

Figure 2.2. Schematic of beamline 9.3.2 rotateable platform. 


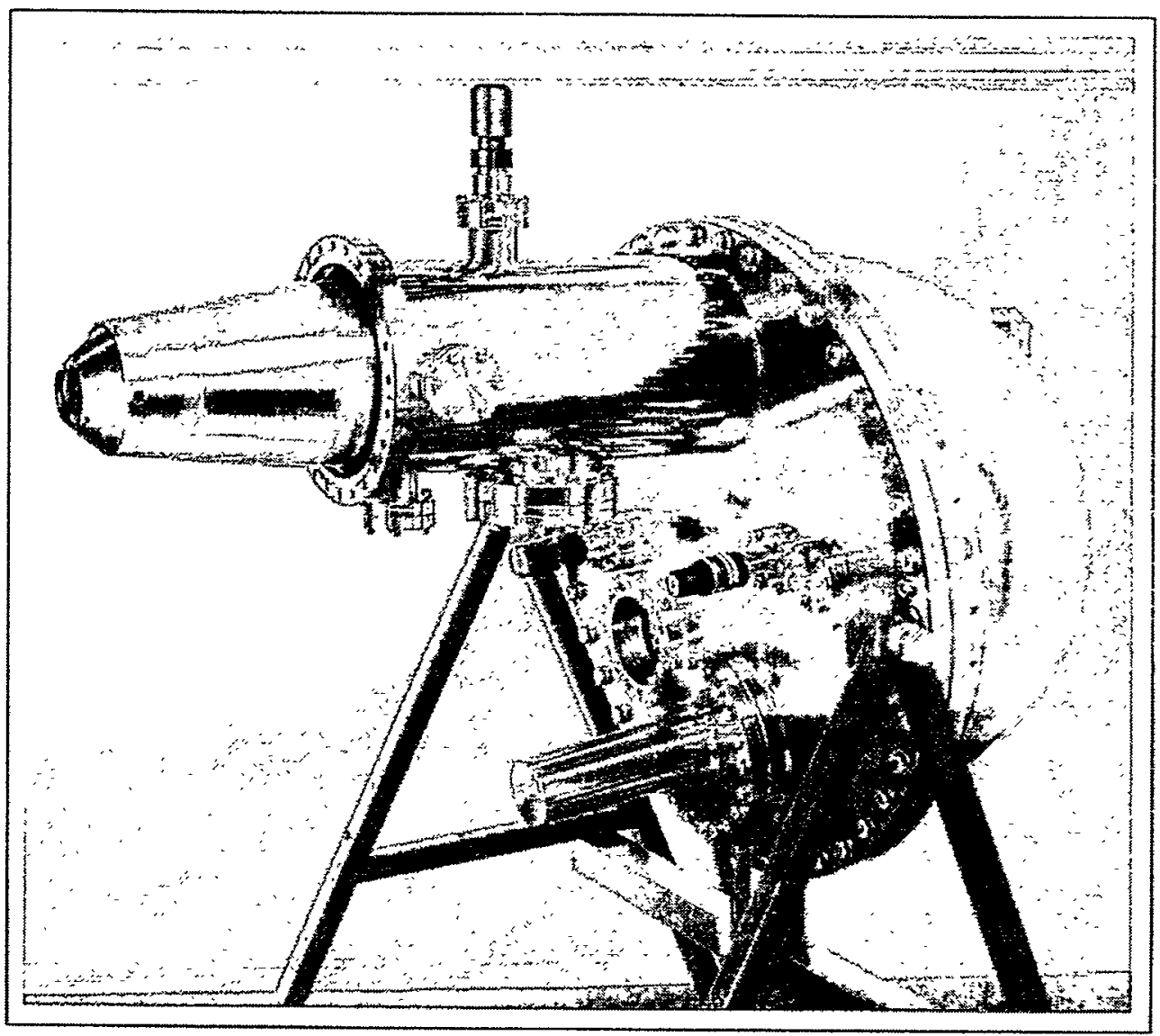

Figure 2.3. Photograph of the Scienta SES-200 electrostatic analyzer. 


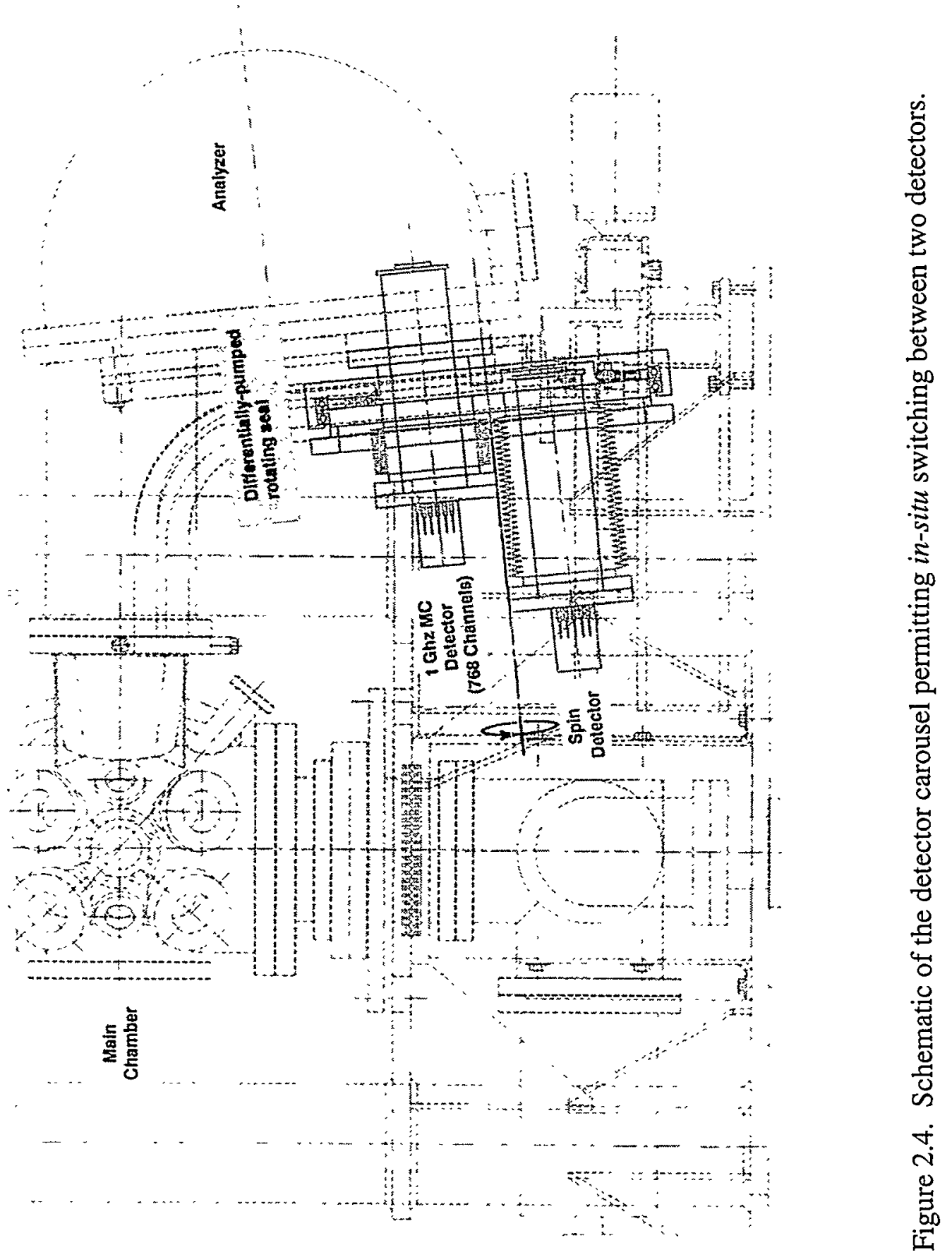




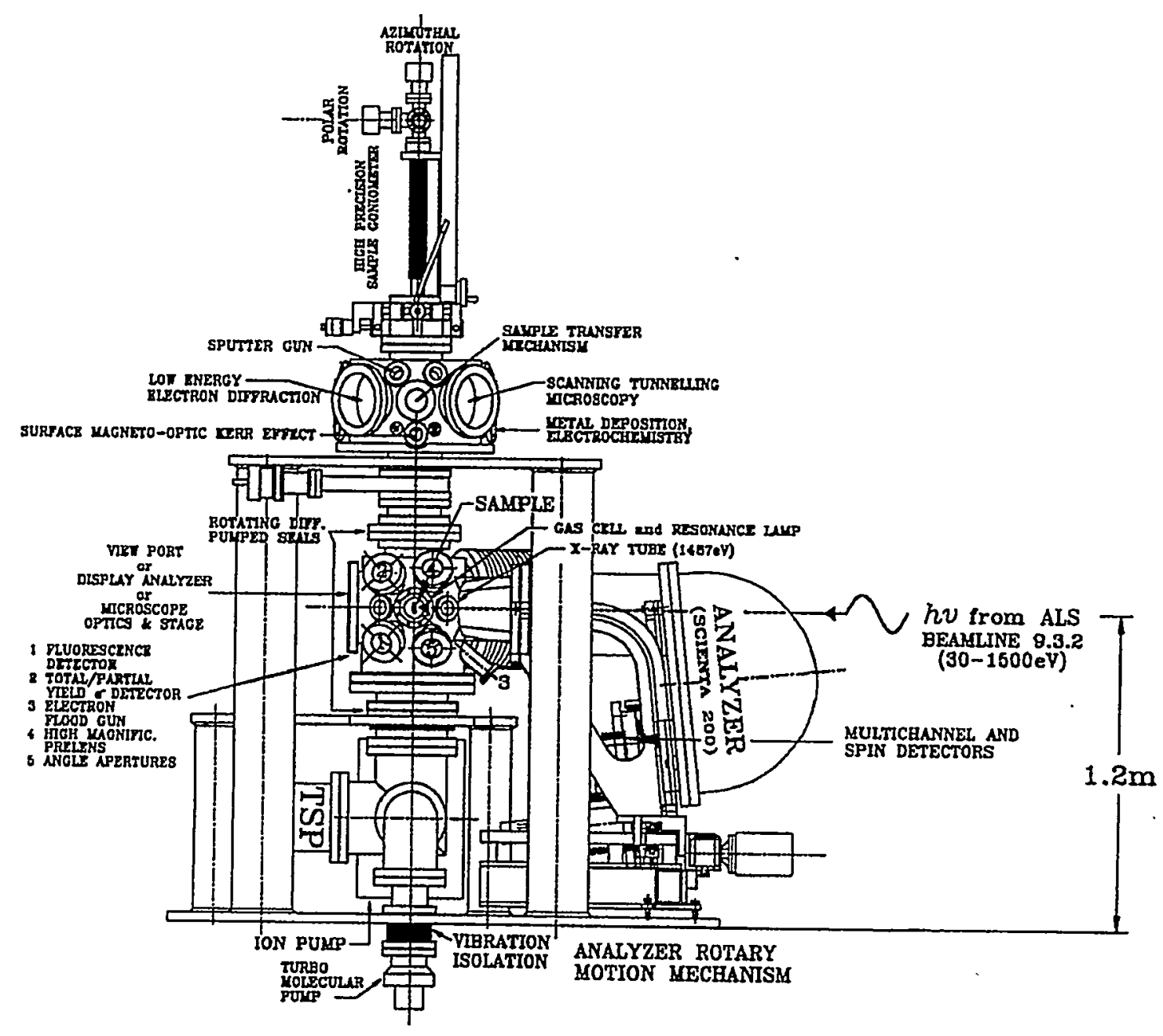

Figure 2.5. Schematic of the Advanced Photoelectron Spectrometer/Diffractometer (APSD). 


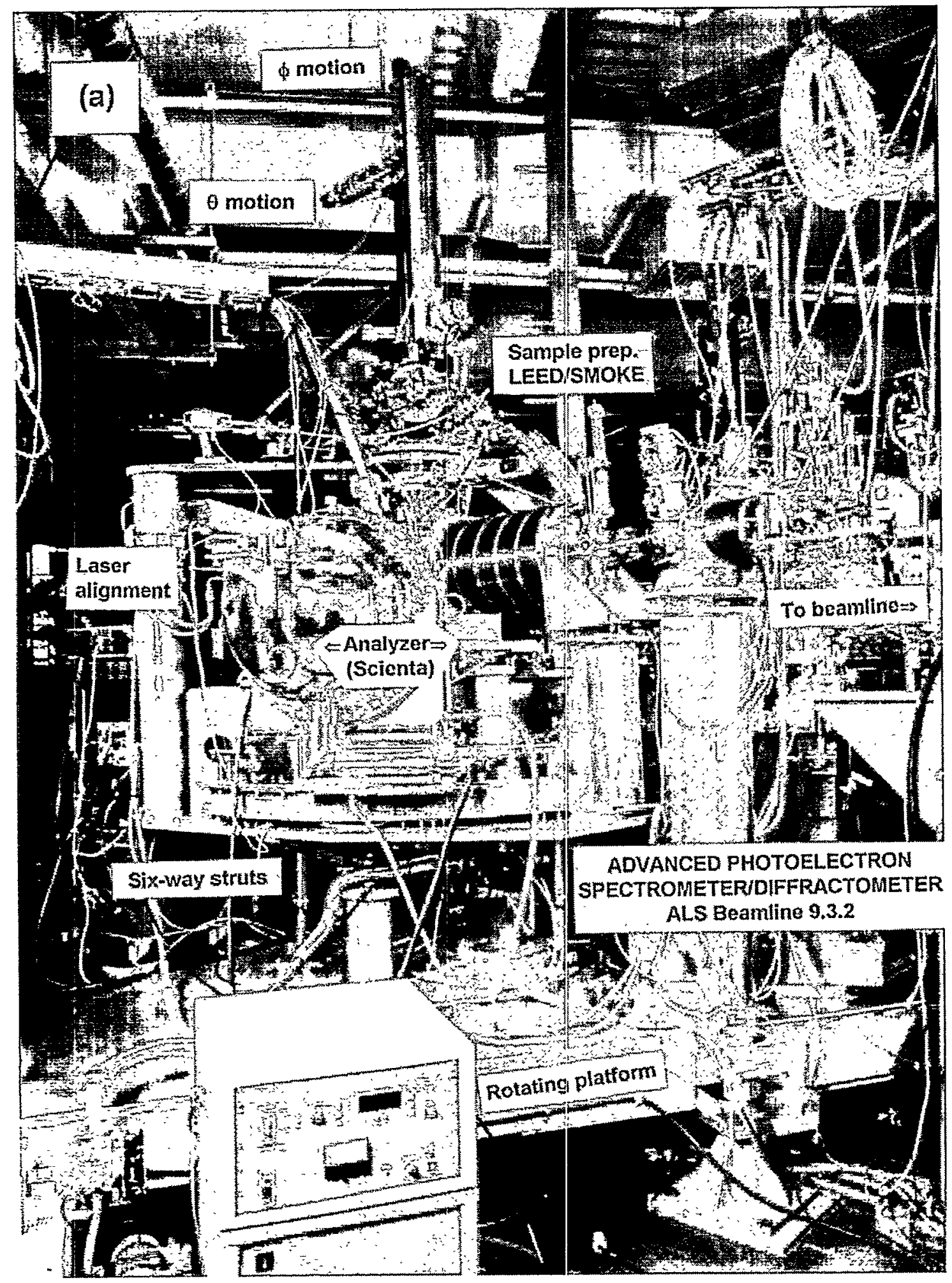

Figure 2.6. Photographs of the Advanced Photoelectron Spectrometer/Diffractometer (APSD) in three views. 


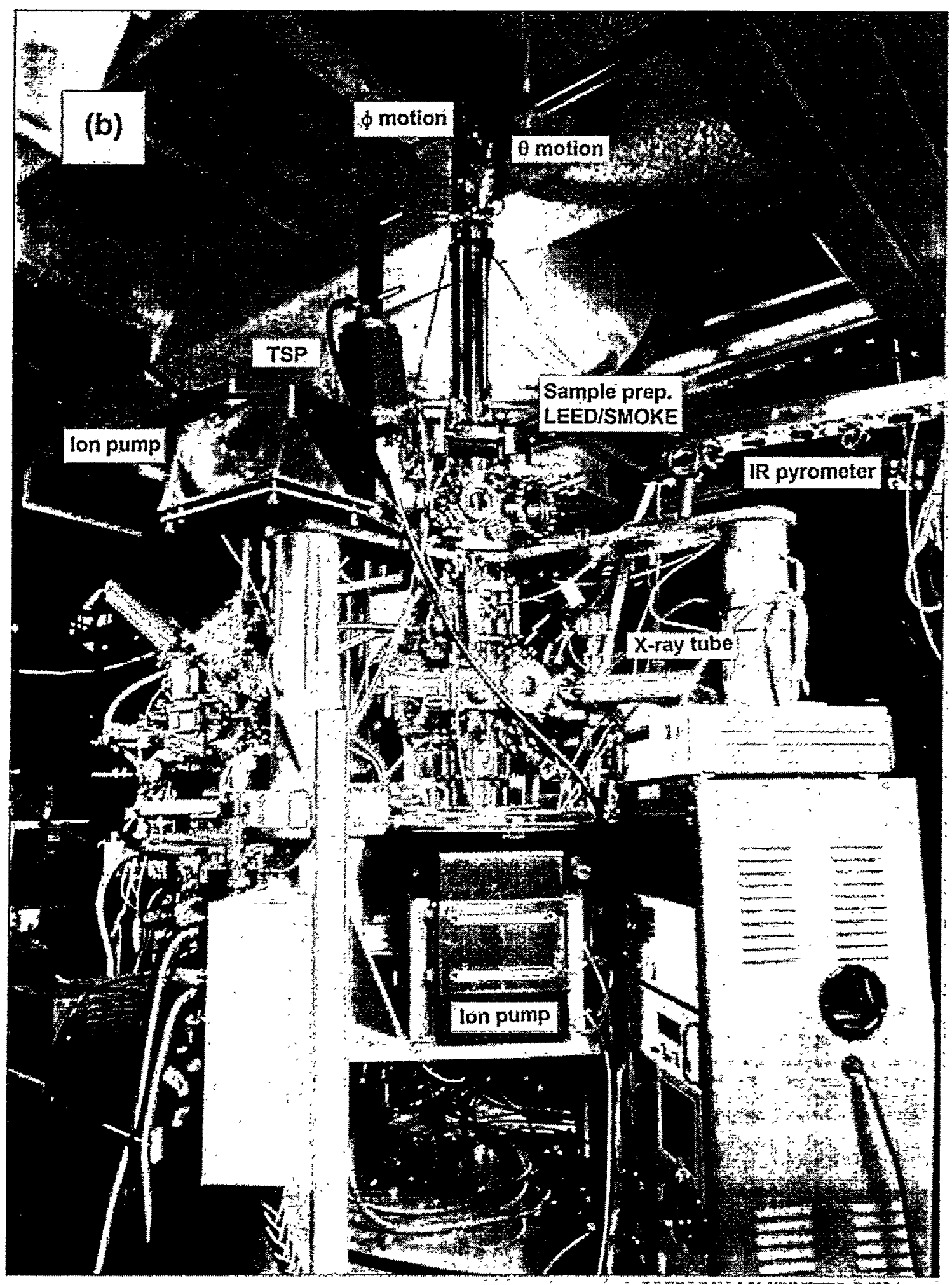




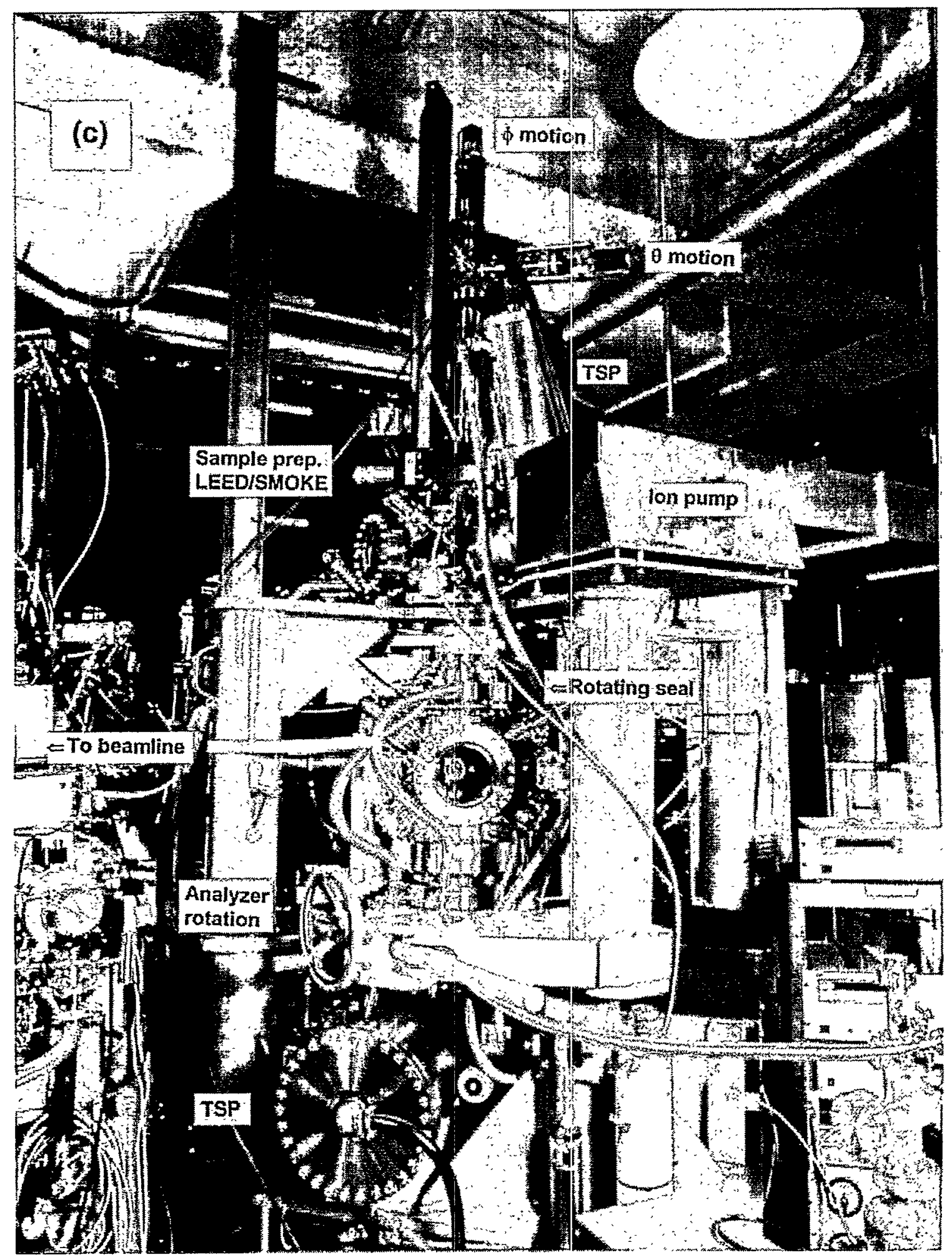




\section{ADVANCED PHOTOELECTRON SPECTROMETER/ DIFFRACTOMETER}

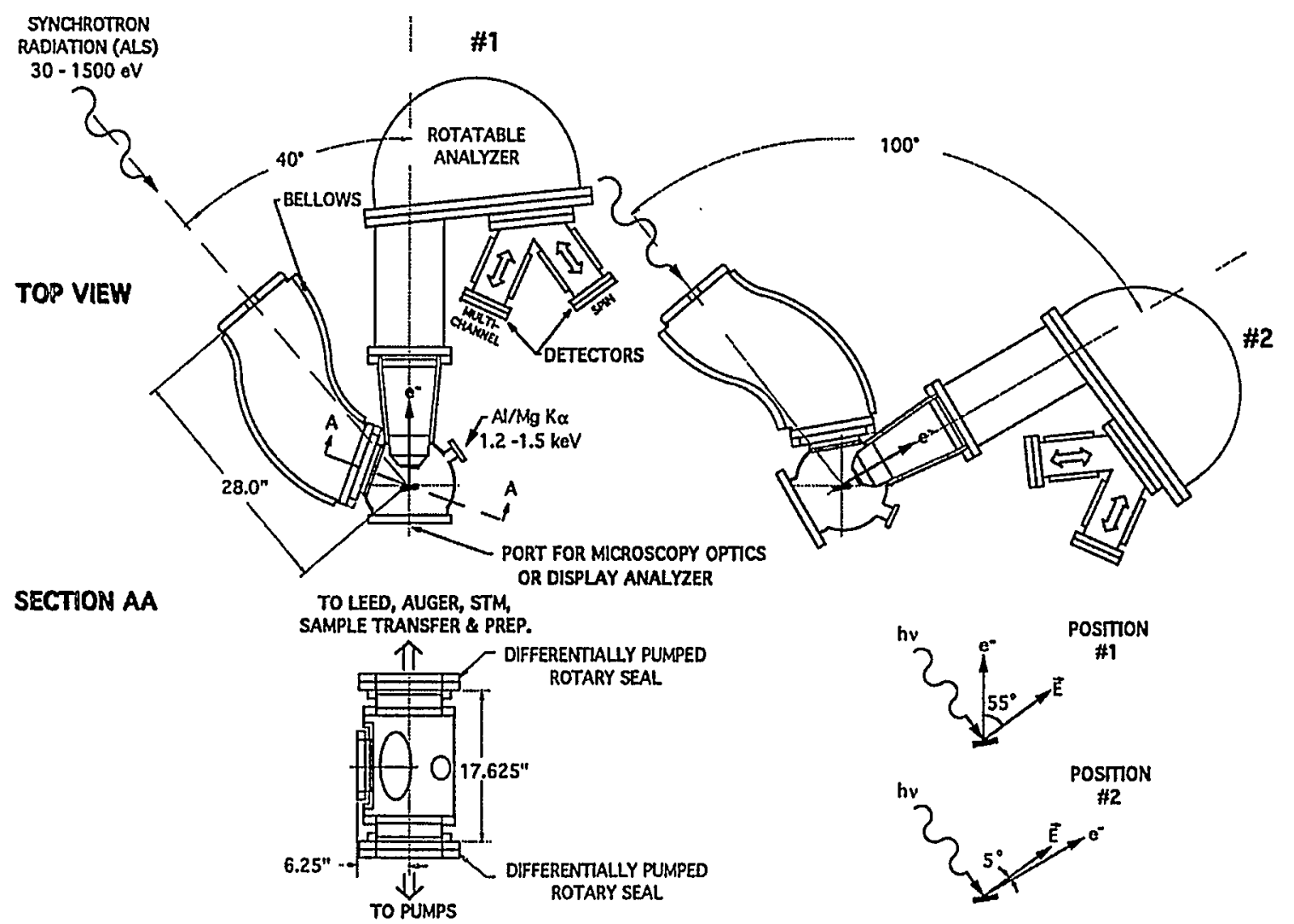

Figure 2.7. Schematic indicating the in-plane rotation of the main analysis chamber that is possible with the APSD. 


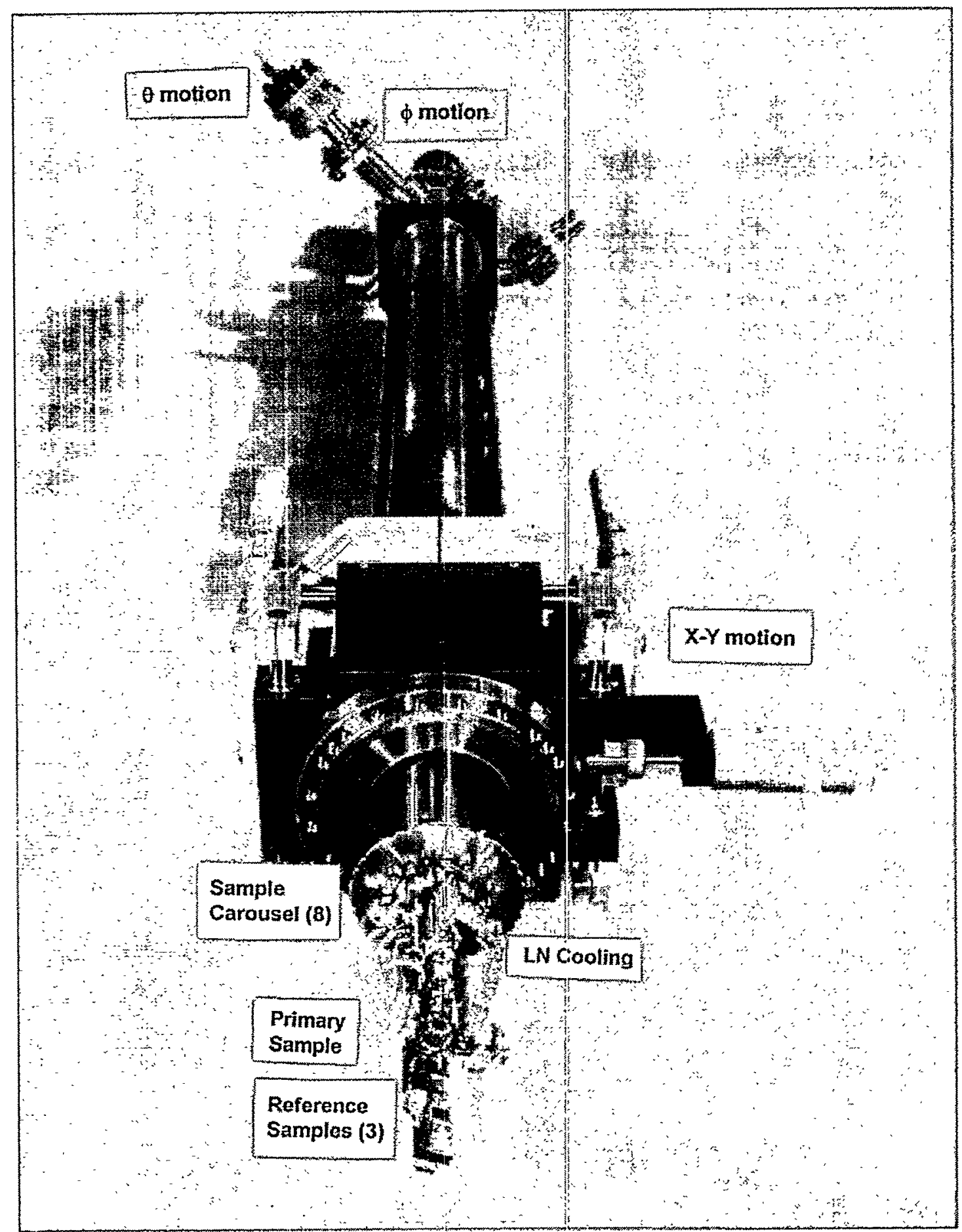

Figure 2.8. Overall photograph of the sample manipulator, showing the precision rotary feedthroughs for $\theta$ and $\phi$ motion, the long bellows for vertical (z) translation, the horizontal $(x-y)$ motions, and various elements near the sample. 


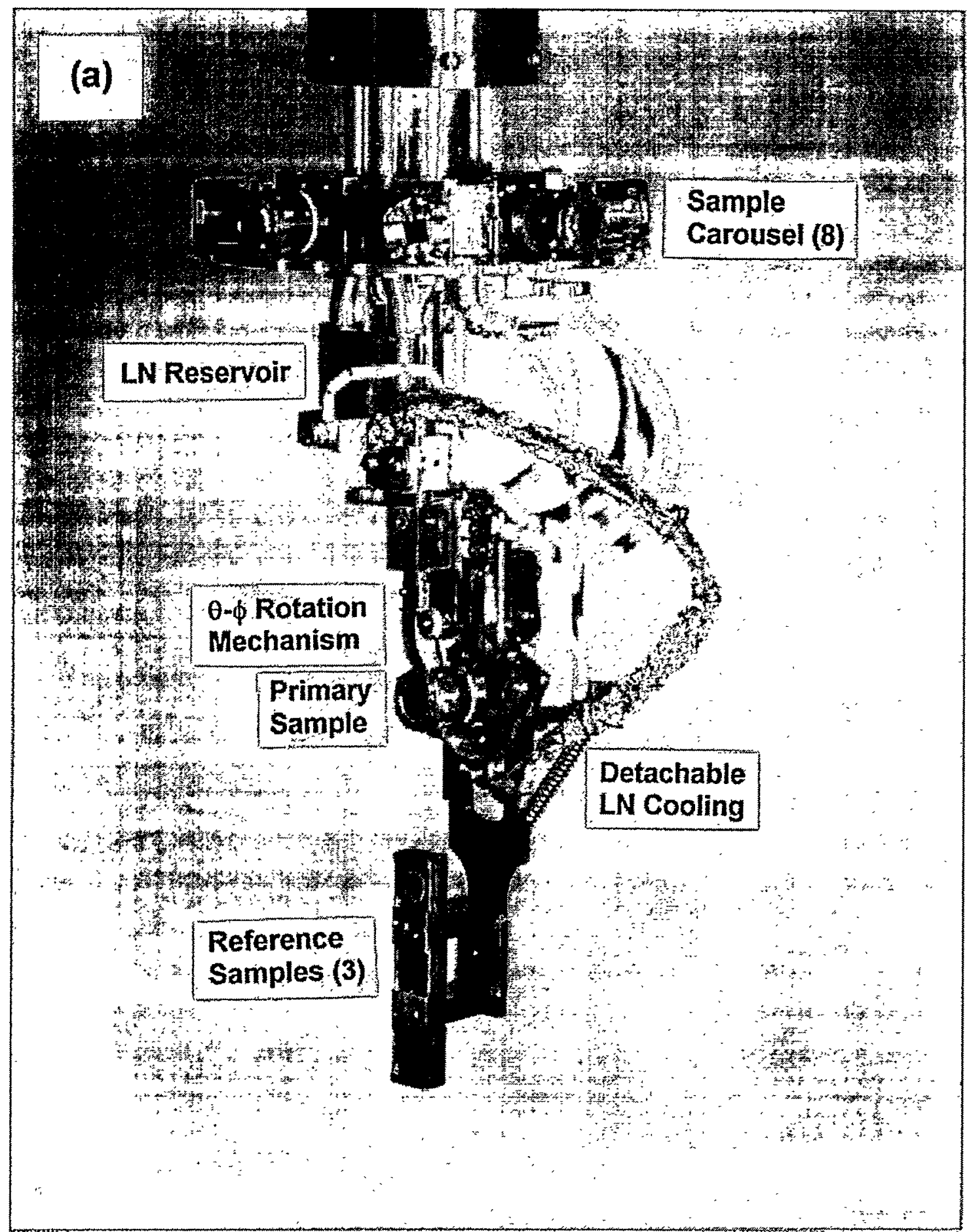

Figure 2.9. Photographs in two views of the two-axis goniometer with sample cooling mechanism and in-situ sample storage carousel. In (b), the placement of the wobble stick sample transfer mechanism is also indicated. 


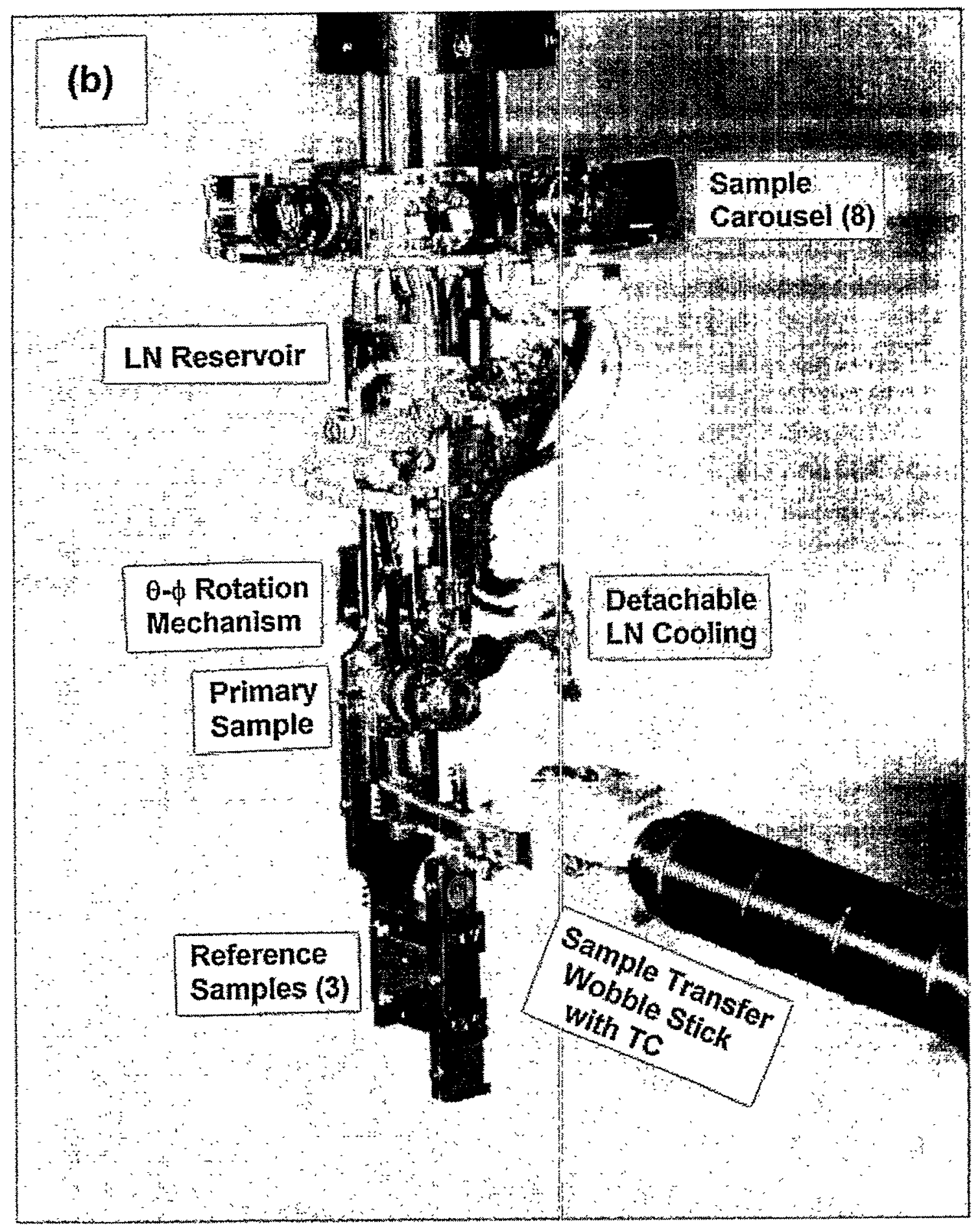




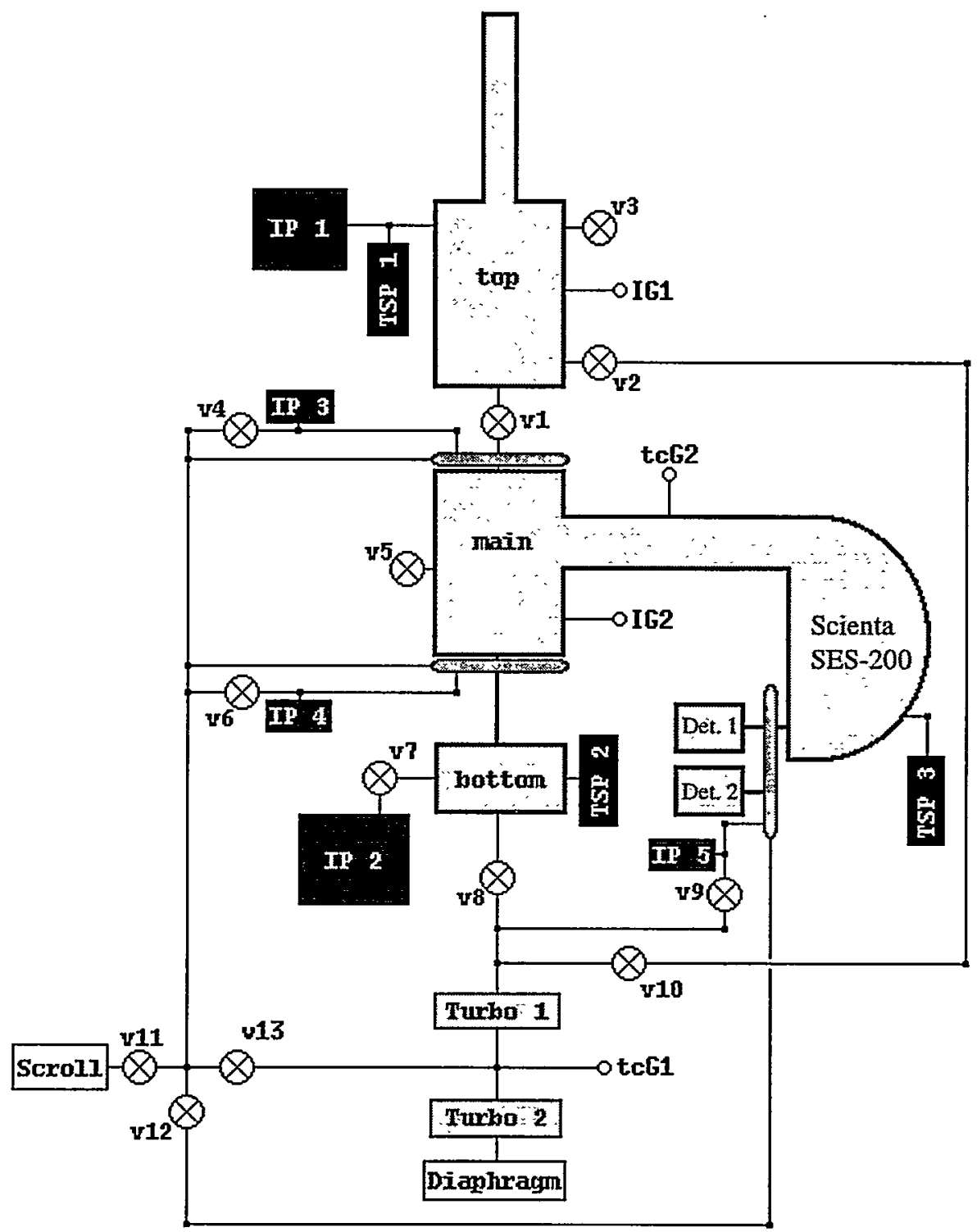

IP - Ion Pump

TSP - Titanium Sublimation Pump

V - Valve

Scroll - Dry Mechanical Scroll Pump
IG - Ion Gauge

tcG - ThermoCouple Gauge

Turbo - Turbomolecular Pump

Diaphragm - Dry Mechanical

Diaphragm Pump

Figure 2.10. Schematic of the pumping and valving system for the APSD. 


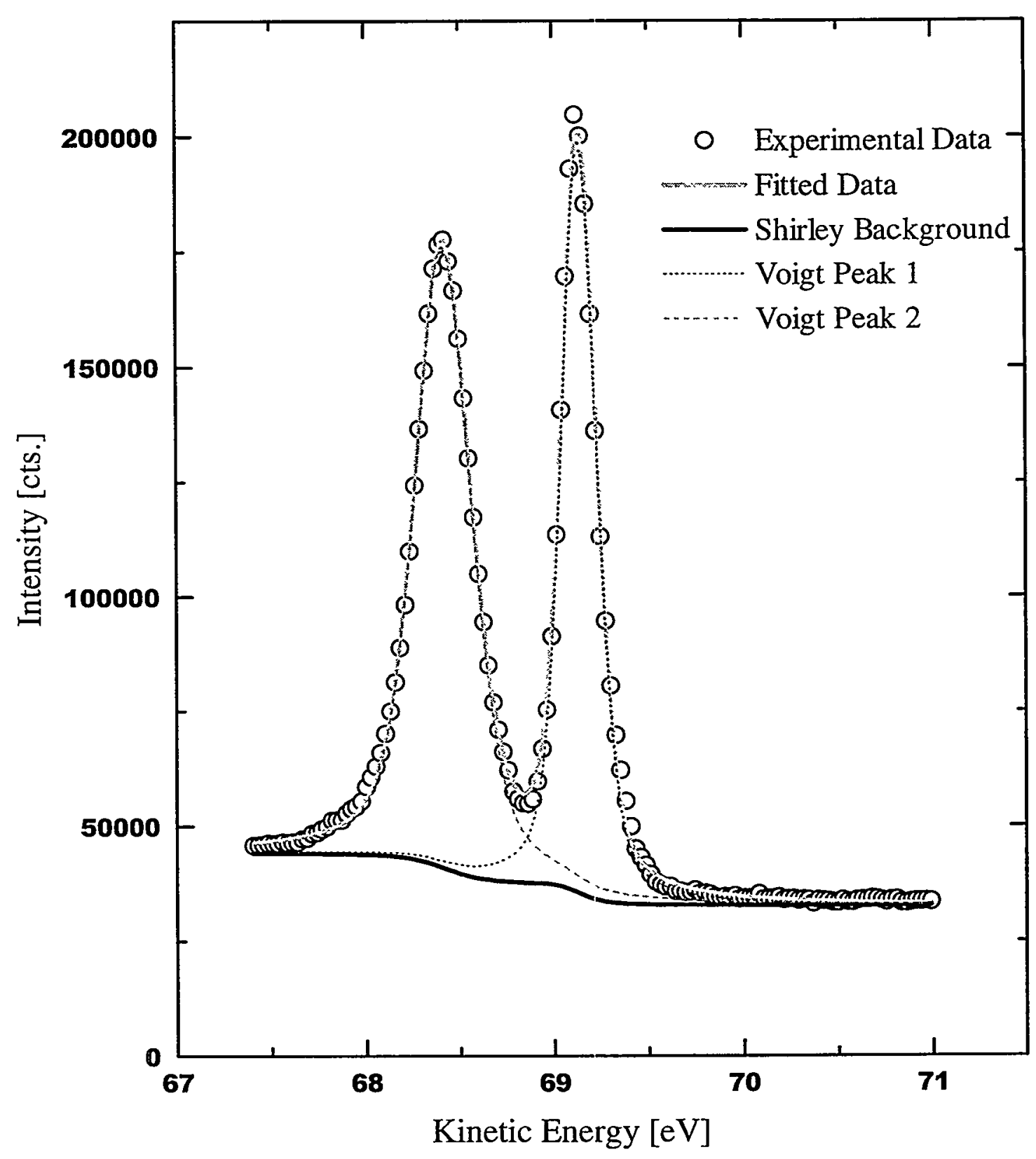

Figure 2.11. W $4 \mathrm{f}$ spectrum of one ML oxygen on $\mathrm{W}(110)$ in a $(1 \times 1) \times 12$ structure, fitted with two Voigt functions and riding on Shirley-type inelastic background. 


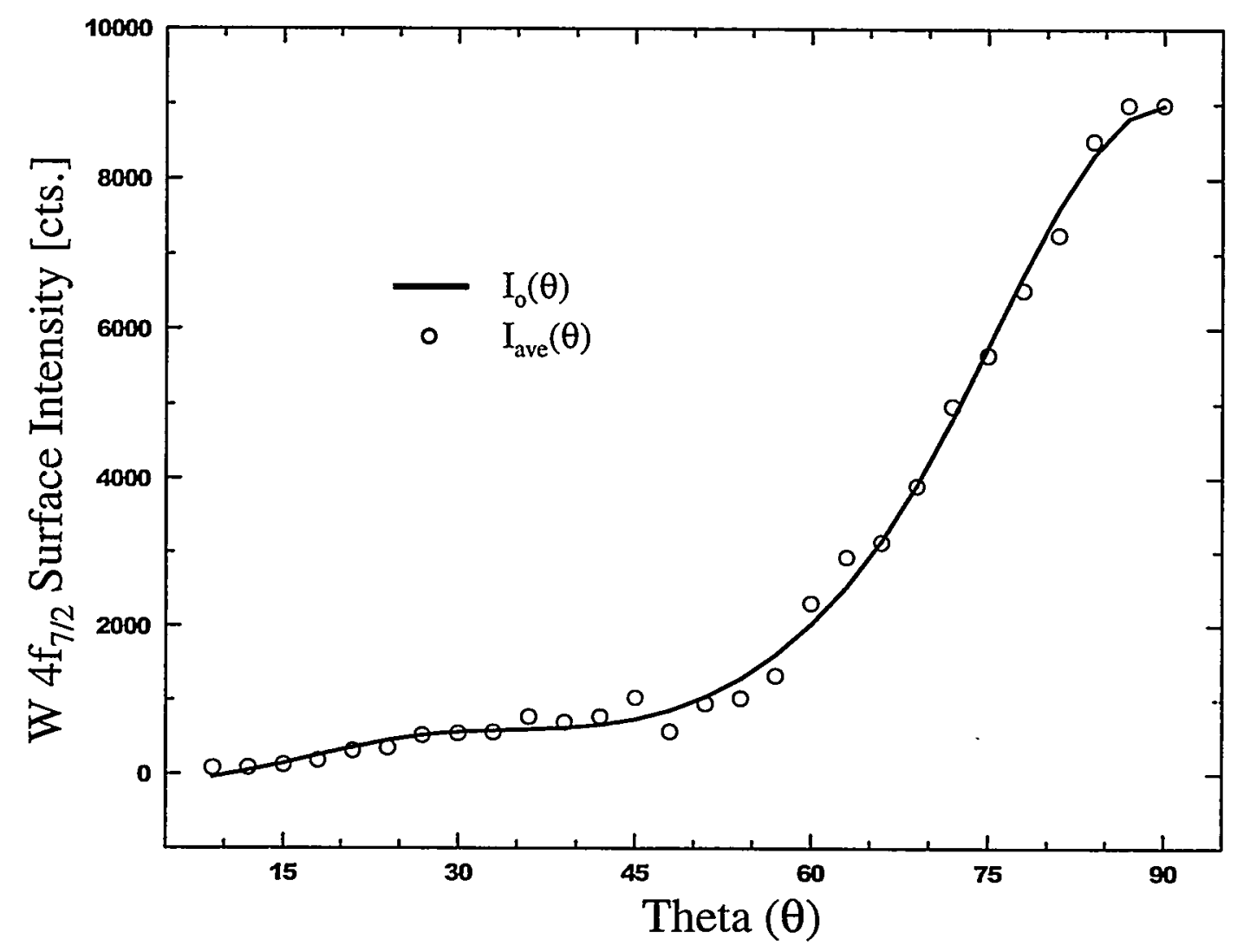

Figure 2.12. Spline fitting of azimuthally-averaged data to derive $I_{0}(\theta)$. The smooth curve is $I_{0}(\theta)$ and the azimuthally-averaged data points are $I_{\text {ave }}(\theta)$. 


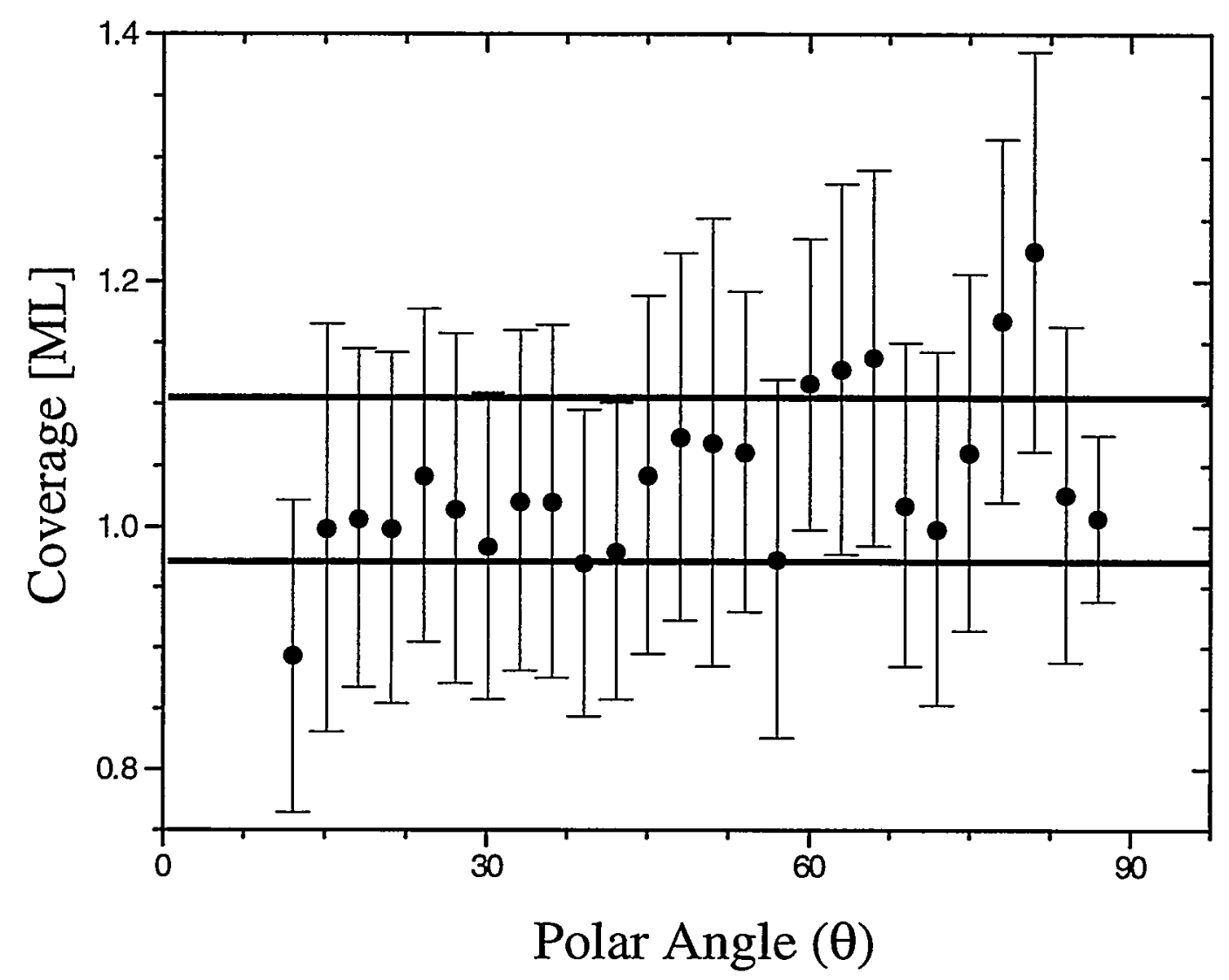

Figure 2.13. Oxygen coverage determination for a saturated coverage of oxygen on $W(110)$ in a $(1 \times 1) \times 12$ structure. The error bars are the standard deviation of the coverage as determined at each polar angle $\theta$, which provide an estimate of the possible error if diffraction is not accounted for. The average coverage is $1.04 \mathrm{ML}$. The solid horizontal lines are at $\pm 0.07 \mathrm{ML}$ (one standard deviation) of the data points. 


\section{Chapter 3}

\section{Full-Solid-Angle Photoelectron Diffraction from \\ Bulk and Surface Atoms of Clean W(110)}

\section{I. INTRODUCTION}

We have studied the atomic structure of the clean W(110) surface by means of site-resolved scanned-angle $\mathrm{W}_{4} \mathrm{f}_{7 / 2}$ photoelectron diffraction $(\mathrm{PD})$ data obtained over nearly the full $2 \pi$ solid angle above the surface. PD is by now a well-developed and in some respects unique tool for studying surfaces that is not only element-specific through the atomic core levels, but also chemical-state and site specific through chemical shifts and surface core level shifts (SCLS's) [1]. However, prior to the availability of highbrightness third-generation synchrotron radiation sources (such as the Advanced Light Source at which this study was performed), fully exploiting the site specificity has not been possible because of the small shifts involved and the resultingly large times needed to obtain sufficiently large high-resolution data sets. In this study, we have measured and analyzed such data sets for the well-characterized W(110) test case, both to demonstrate what is possible with these new excitation sources and to make a more rigorous test of the multiple scattering methodology that has been developed for simulating such data.

The clean W(110) surface was chosen as a well-studied reference case [2-9] to check the accuracy of structure determinations from such scanned-angle data via $\mathrm{R}$-factor comparisons of experiment with theoretical multiple scattering calculations. This system was selected for several reasons: First, the surface crystallography is well established, 
with the general conclusion that there is very little or no relaxation of the interplanar distances from those in the bulk [4-8]. Second, the surface core level shift is relatively large at $\sim 320 \mathrm{meV}$ and the surface and bulk linewidths are relatively narrow: thus the spectra are easily fitted with two components so as to derive accurate intensities $[3,9]$. (Surface core-level shifts have been known for some time, and various discussions of them and their physical origins appear elsewhere [11-13].) Finally, cleaning the W(110) single crystal is relatively easy and very reproducible. In this work, a photoelectron kinetic energy of $\sim 40 \mathrm{eV}$ was used. This energy was lower than in most prior PD studies [4], and it leads to greater surface sensitivity, but also provides further challenges to theory, with both elastic and inelastic scattering expected to be very strong. In our analysis of the data, we have also assessed the influence of various non-structural theoretical input parameters on the final structure, such as electron inelastic attenuation length, scattering phase shifts, and inner potential. A final optimized structure is presented, together with comparisons to prior studies by PD and low energy electron diffraction [4-6,8] and comments on the future applications of this method to other unknown structures.

\section{II. EXPERIMENTAL METHODOLOGY}

These experiments were performed on bend-magnet beamline 9.3.2 at the ALS [10(a)] using its advanced photoelectron spectrometer/diffractometer experimental station [10(b)]. The end station incorporates a Scienta SES200 hemispherical electrostatic analyzer that was used to obtain all spectra. The sample was prepared by an initial 
oxygen treatment at a temperature of $1200^{\circ} \mathrm{C}$ and a pressure of $1 \times 10^{-7}$ Torr $\mathrm{O}_{2}$ to reduce the amount of carbon from the near-surface region. This was followed by repeated flashing at up to $2200^{\circ} \mathrm{C}$ by electron bombardment heating. Sample cleanliness was then monitored by $\mathrm{x}$-ray photoelectron spectroscopy (XPS) using Al K $\alpha$ excitation, low energy electron diffraction (LEED), and most sensitively by the surface core level shift (SCLS) between the bulk and surface atoms, which reaches a maximum value of about $-320 \mathrm{meV}$ for a fully clean surface, but decreases in magnitude with time due to exposure to the residual gas in ultrahigh vacuum [3(b),9]. The greatest contribution to this decrease is hydrogen adsorption from the residual gas. Fig. 3.1 shows (a) the schematic experimental geometry, (b) the atomic geometry to be determined, and (c) a typical spectrum. For a given sample preparation, the actual data collection time was limited to 30 minutes to maintain adequate cleanliness. The base pressure during measurements was $1.3 \times 10^{-10}$ Torr.

The photoelectrons were excited by $70 \mathrm{eV}$ linear-polarized light from a sphericalgrating monochromator, as second-order light from a $35 \mathrm{eV}$ setting [10(a)], with the polarization $\varepsilon$ lying in the plane containing also the [110] surface normal and the photoelectron wave vector $k$ (cf. Fig. 3.1(a)). This resulted in $W 4 f_{7 / 2}$ photoelectrons at a kinetic energy of $\sim 39-40 \mathrm{eV}$, as shown in Fig. 3.1(c); the bulk and surface components are easily resolvable. The angle ( $\alpha$ ) between the incident radiation and the analyzer (which is in fact variable for this system [10(b)]), was for this experiment fixed at $70^{\circ}$. 
To enable the most accurate measurement of the bulk and surface peak intensities, full $\mathrm{W} 4 \mathrm{f}_{7 / 2}$ spectra were obtained at each direction and stored for later analysis by peak fitting. Data were obtained over the nearly full $2 \pi$ solid angle above the surface by rotating the sample on two perpendicular axes for polar takeoff angle $\theta$ (measured with respect to the surface) and azimuthal angle $\phi$ (measured with respect to the [1]0] direction). The data consisted of twenty-eight azimuthal scans spanning takeoff angles $\theta$ from $9^{\circ}$ to $90^{\circ}$ (=normal emission) and with $3^{\circ}$ steps in takeoff angle. The azimuthal step size (which had a minimum value of $3^{\circ}$ for $\theta=9^{\circ}$ ) was adjusted upward with increasing takeoff angle such that the final data set consisted of 892 spectra representing roughly equal solid-angle increments. Angles below $\theta=9^{\circ}$ were not used due to the much lower count rates and thus longer counting times involved. Due to the symmetry of the crystal surface, only a $90^{\circ}$ azimuthal scan is in principle needed to provide information on the full $360^{\circ}$ intensity profile. However, to increase accuracy and average over any small sample misalignments, we collected data over $180^{\circ}$ in azimuth, and then mirror-averaged this into a $90^{\circ}$ sector that was finally used to generate full $360^{\circ}$ representations of our data. The solid angle cone accepted by the analyzer has a half angle of $\sim 5^{\circ}$. The brightness of the light from the beamline, together with the high luminosity of the Scienta analyzer for a given resolution setting, permitted taking a single high-resolution spectrum in 20 seconds; thus the actual data taking time was about 5 hours. With further improvements in detection and the use of undulator radiation for excitation, it is expected that this time will be reduced by at least an order of magnitude in the future [10(b)]. Any variations of radiation flux with time were normalized out in determining the final 
intensities $\mathrm{I}(\theta, \phi)$. Each spectrum was resolved into bulk and surface components by fitting them with asymmetric Voigt functions of at first variable widths and spacings, riding on a Shirley-type inelastic background; in the final fits, the widths and spacings were kept fixed at their average values over the whole set of data to minimize spurious intensity changes. The two intensities so derived thus permit deriving site-specific diffraction patterns from both the tungsten atoms in the surface layer and the tungsten atoms below the surface layer. Finally, these two intensities were normalized into a chi function $\chi(\theta, \phi)=\left[I(\theta, \phi) .-I_{0}(\theta)\right] \Lambda_{0}(\theta)$, with $I_{0}(\theta)$ being the estimated intensity in the absence of diffraction effects. $I_{0}(\theta)$ was determined by first averaging $I(\theta, \phi)$ over each $\phi$ value to produce $\bar{I}(\theta)$, and then fitting $\bar{I}(\theta)$ with a spline function in $\theta$ to finally yield $I_{0}(\theta)$. Figs. 3.2(a) and 3.2(c) show the final separate diffraction patterns $\chi_{\text {bulk }}$ and $\chi_{\text {surface }}$, respectively. Due to the different local geometries around the two types of atoms, the bulk and the surface diffraction patterns are strikingly different.

\section{III. RESULTS AND DISCUSSION}

The $39-40 \mathrm{eV}$ kinetic energy range of the photoelectrons used here is very near the minimum in their inelastic attenuation length $\Lambda_{\mathrm{e}}$, which we have estimated from an analysis of the takeoff-angle dependence of the experimental surface/bulk intensity ratio to be only 2.0-4.0 $\AA$. In this analysis, the $\mathrm{I}_{o}(\theta)$ curves for both surface and bulk were determined as indicated above, and the resulting $\theta$-dependent ratio $I_{0}(\theta)_{\text {surface }} / I_{0}(\theta)_{\text {bulk }}$ analyzed via a standard formula for the quantitative analysis of angular-dependent peak 
intensities for an attenuating thin overlayer (the surface layer of atoms) on a semi-infinite substrate (the bulk atoms) [14]:

$\frac{I_{\mathrm{o}}(\theta)_{\text {surface }}}{I_{\mathrm{o}}(\theta)_{\text {bulk }}}=\frac{\left[1-\exp \left(-\frac{d_{110}}{\Lambda_{\mathrm{e}} \sin \theta^{\prime}}\right)\right]}{\exp \left(-\frac{d_{110}}{\Lambda_{\mathrm{e}} \sin \theta^{\prime}}\right)}=\exp \left(\frac{d_{110}}{\Lambda_{\mathrm{e}} \sin \theta^{\prime}}\right)-1$

or

$$
\Lambda_{\mathrm{e}}=\frac{\mathrm{d}_{110}}{\sin \theta^{\prime} \cdot \ln \left(1+\frac{\mathrm{I}_{\mathrm{o}}(\theta)_{\text {surface }}}{\mathrm{I}_{\mathrm{o}}(\theta)_{\text {bulk }}}\right)}
$$

where $\theta^{\prime}$ is the angle of propagation of the electron inside the surface before refraction at the potential barrier $V_{o}$, and $d_{110}$ is the (110) interplanar spacing $=2.23 \AA$. Thus, $\Lambda_{e}$ could be determined from Eq. 3.1 as a function of $\theta$. The application of this formula to our data gives the curves shown in Fig. 3.3, where three different values of the inner potential $\mathrm{V}_{\mathrm{o}}$ between 10 and $15 \mathrm{eV}$ have been used to determine the internal angle of propagation $\theta$ ' before surface refraction to yield the externally observed angle $\theta$. The values found for $\Lambda_{e}$ are not particularly sensitive to the choice of $V_{0}$, and range from a very low value of about $2.2 \AA$ at normal emission $\left(\theta=90^{\circ}\right)$ to a high of about 4.5 to 5.2 $\AA$ at the lowest takeoff angle of $\theta=9^{\circ}$. The minimum value thus corresponds to an attenuation length about equal to the (110) interplanar spacing. Prior analyses of this type [14] further suggest that the most reliable range of angles (i.e., for which the assumptions implicit in Eq. 3.1 are most valid) is nearer normal emission, or over about $\theta=45^{\circ}$ to $90^{\circ}$ [14], which yields approximately $2.2-4.0 \AA$ as a final best estimate from this 
approach. For comparison, this is somewhat lower than the approximately $6.0 \AA$ predicted by the so-called Universal Curve derived by Seah and Dench using a fit to experimental attenuation lengths for several solid elements [15]; this fit describes the attenuation length via the formula $\Lambda_{e}=\mathrm{C}_{1} \mathrm{E}^{-2}+\mathrm{C}_{2} \mathrm{E}^{1 / 2}$, with $\mathrm{C}_{1}$ and $\mathrm{C}_{2}$ being empirical constants. However, the determination of Eq. 3.1 and Fig. 3.3 is certainly more quantitatively applicable to our particular case. A more exact material-dependent calculation for the attenuation length due to Tanuma, Powell, and Penn and known as the TPP-2M formula [16] is not applicable below $50 \mathrm{eV}$, but in any case yields a value of 5.2 $\AA$ right at $50 \mathrm{eV}$ that is very close to our other estimates. Thus, we have finally used a value of $3.0 \AA$ in our multiple scattering calculations, although we have verified that values over the range of 3-6 $\AA$ did not significantly alter the final theoretical diffraction patterns, nor change the final structural conclusions after R-factor analysis. The very small values found for the experimental attenuation lengths enhance the surface contribution, making it stronger than the bulk contribution for many emission directions, as illustrated in Fig. 3.1(c).

In order to now determine the atomic structure, multiple scattering diffraction calculations were performed using a program developed by Chen et. al. [17] and based on the convenient Rehr-Albers separable-Green's-function that has been used previously for treating the multiple scattering of photoelectrons [18]. Because of its high atomic number, tungsten is a very strong scatterer, particularly for low-energy electrons $[6,19]$. Thus, several different sets of calculated scattering phase shifts were tried. Each set resulted in very similar scattering factors, and the different choices did not influence our 
final structural conclusions, as discussed further below. However, in what follows, we show results obtained from two sets of phase shifts to illustrate this point. Due to the very strong inelastic attenuation, only emission from the top 3-4 layers was found to contribute significantly to the diffraction, but to insure convergence as to cluster size, 5 layers with a total of 101 atoms which have the same symmetry as clean W(110) were used. To describe vibrational effects, the calculations included Debye-Waller factors based on uncorrelated atomic motion, with a Debye temperature of $400 \mathrm{~K}$ for tungsten and room temperature $(298 \mathrm{~K})$ for measurement. The inner potential $V_{0}$, which induces at the surface both a direction change due to refraction and a wavelength change due to the increase in internal kinetic energy, was initially estimated by adding the W(110) work function to the difference between the theoretical values for the muffin-tin zero and the Fermi energy, which yields $13 \mathrm{eV}$. We have further optimized $\mathrm{V}_{\mathrm{o}}$ for the best fit to our data, which yields the very similar value of $12.5 \mathrm{eV}$. This is also the same value as the inner potential used in a recent LEED analysis of W(110) [5], but slightly greater than the $\mathrm{V}_{\mathrm{o}}=10 \mathrm{eV}$ of an earlier LEED analysis for this system [6]. The angular aperture was set to \pm 3 degrees in our calculations, although the actual experimental angular aperture was approximately \pm 5 degrees. We have in addition gaussian-broadened the theoretical calculations so as to have an effective angular aperture of \pm 5 degrees but this was not found to influence the optimum position for the surface atomic layer.

Experiment was compared to theory via a set of five R-factors originally suggested for LEED analyses [21(a)] and later modified for PD analyses [21(b)]; these involve the absolute value of the difference between experimental and theoretical 
intensities (R1), the absolute value squared of this difference (R2), the fraction of the data range over which experiment and theory have different slopes (R3), the absolute value of the difference between the derivatives with azimuthal angle of experimental and theoretical intensities (R4), and the absolute value squared of this difference of derivatives (R5). The trial atomic geometries in theory involved a series of outward relaxations or inward contractions of the surface layer with respect to the second layer below, with the relevant distance being defined as $\delta z$ and measured relative to the unrelaxed bulk interlayer distance of $2.23 \AA$ (cf. geometry in Fig. 3.1(b)). Fig. 3.4 shows the variation with $\delta \mathrm{z}$ of these five $\mathrm{R}$-factors and a normalized sum of these five. The two curves (black circles and grey squares) represent calculations with two different sets of scattering phase shifts (one from a recent LEED calculation [5(b)] and one from a program due to Chen et al. and based on a muffin-tin model of the crystal potential, as in the LEED work [17]). All five $\mathrm{R}$ factors and the normalized sum are found to yield very nearly the same structure, with the overall conclusion being a small $0.10 \pm 0.05 \AA$ outward relaxation of the surface layer from its normal bulk termination. This is thus $4.5 \%$ of the bulk interlayer distance of $2.23 \AA$. The excellent final agreement between experiment and theory for surface emission is illustrated in Figs. 3.2(c) and (d). The analogous comparison for bulk emission in Figs. 3.2(a) and (b) shows good agreement, with the multi-layer nature of the emission and the strong elastic and inelastic scattering involved perhaps accounting for the fact that agreement is not as good as that for surface emission. 
Another interesting aspect of the surface-atom analysis is that theory and experiment are in general in very good agreement if only the surface layer of scatterers is included in the calculation (i.e., if no bulk scatterers are present); this is represented by the horizontal lines in Fig. 3.4. This implies that intralayer scattering is the most important mechanism producing the observed diffraction features. However, with a proper calculation including all scatterers, the influence of the bulk scatterers is evident: the minimum R-factors are slightly lower for the optimum geometry, and the values for interlayer distances more than about $0.1 \AA$ away from the optimum in either direction are significantly higher, such that the agreement decreases.

Our final structure agrees with prior PD results by Jugnet et al. based on a much smaller scanned-angle data set, which show no relaxation to within a larger estimated error of $\pm 0.2 \AA[4]$. Scanned-energy PD by Kim et al. show a slight outward relaxation of $0.03 \pm 0.05 \AA[8]$. Our structure is also at least in qualitative agreement with prior LEED studies of this surface, which have reached conclusions varying from a small contraction of $0.07 \AA[5]$ to no relaxation at all [6,7]. However, the sign of the relaxation as we find it is opposite to the small average negative value found in LEED.

Finally, Fig. 3.5 shows R-factors versus $\delta$ z over an expanded scale and illustrates the oscillatory nature of such fits, a phenomenon familiar in LEED [22] and also seen before in PD [21(b)]. But in all cases, the deepest minimum is that for the structure we have discussed above. Nonetheless, this kind of plot makes it clear that using some sort of direct first-approximation such as a holographic inversion of $\mathrm{PD}$ data [1(b)] could 
serve as a useful adjunct to narrow the structure search interval, thus avoiding local minima that may not represent the true structure.

\section{IV. CONCLUSIONS}

Full-solid-angle photoelectron diffraction has been applied to the well-defined test case of the clean W(110) surface, for which the surface and bulk diffraction patterns can be measured separately. Multiple scattering theory is found to provide an excellent description of the surface data, even at a low kinetic energy of $\sim 40 \mathrm{eV}$ for which both elastic and inelastic scattering are expected to be very strong, and R-factor comparisons of experiment and theory permit deriving structural information with an accuracy of about $\pm 0.05 \AA$. Our results for this structure are in excellent agreement with prior photoelectron diffraction studies based on more limited data sets, showing a slight $4.5 \%$ outward relaxation of the surface W layer; our structure shows more outward relaxation than in prior LEED analyses, but is not in strong disagreement with them. Our data also permit estimating an effective inelastic attenuation length in $\mathrm{W}$ at $40 \mathrm{eV}$ of about 2-4 $\AA$. Future applications of such large-scale site-resolved data sets to various surfaces and interfaces are thus promising, with two recent examples being to a metal-metal interface $(\mathrm{Fe} / \mathrm{W}(110))[23]$ and the structures of oxygen on $\mathrm{W}(110)[20]$. 


\section{References:}

[1] (a) C. S. Fadley in "Synchrotron Radiation Research: Advances in Surface and Interface Science", R. Z. Bachrach, Ed. (Plenum Press, New York, 1992); (b) C.S. Fadley, Y. Chen, R.E. Couch, H. Daimon, R. Denecke, J.D. Denlinger, H. Galloway, Z. Hussain, A.P. Kaduwela, Y.J, Kim, P.M. Len, J. Liesegang, J. Menchero, J. Morais, J. Palomares, S.D. Ruebush, E. Rotenberg, M. B. Salmeron, R. Scalettar, W. Schattke, R. Singh, S. Thevuthasan, E.D. Tober, M.A. Van Hove, Z. Wang, and R.X. Ynzunza, Prog. Surf. Sci. 54, 341 (1997).

[2] D. Spanjaard, C. Guillot, M.C. Desjonquéres, G, Tréglia, and J. Lecante, Surf. Sci. Reports 5, 1 (1985).

[3] (a) Tran Minh Duc, C. Guillot, Y. Lassailly, J. Lecante, Y. Jugnet, and J.C. Vedrine, Phys. Rev. Lett., 43, 789 (1979); (b) D.M. Riffe, G.K. Wertheim, and P.H. Citrin, Phys. Rev. Lett., 63, 1976 (1989); D.M. Riffe and G.K. Wertheim, private communication.

[4] Y.Jugnet, N.S. Prakash, L. Porte, Tran Minh Duc, T.T.A. Nguyen, R. Cinti, H.C. Poon and G. Grenet, Phys. Rev. B, 37, 8066 (1988).

[5] (a) M. Arnold, S. Sologub, G. Hupfauer, P. Bayer, W. Frie, L. Hammer, and K. Heinz, Surf. Rev. Letts., 4, 1291 (1997). (b) G. Schmidt, H. Zagel, H. Landskron, K. Heinz, K. Mueller, and J.B. Pendry, Surf. Sci. 271, 416 (1992). 
[7] J.C. Buchholz, G.C. Wang, and M.G. Lagally, Surf. Sci. 49, 508 (1975).

[8] B.Kim, J.Chen, J.L. Erskine, W.N.Mei, C.M.Wei, Phys.Rev.B 484735 (1993).

[9] D.M. Riffe, G.K. Wertheim, and P.H. Citrin, Phys. Rev. Lett. 65, 219 (1990).

[10] (a) Z. Hussain, W. R. A. Huff, S. A. Keller, E. J. Moller, P. A. Heimann, W. McKinney, H. A. Padmore, C. S. Fadley, and D. A. Shirley, J. Elec. Spec. and Rel. Phen. 80, 401 (1996) and abstract by E.J. Moler et al. in the Advanced Light Source Compendium of Users Abstracts and Technical Reports, 1993-1996, Report LBNL-39981, UC-411, p. 336; (b) C.S. Fadley, M.A. Van Hove, Z. Hussain, and A.P. Kaduwela, J. Elec. Spec. and Rel. Phen. 75, 273 (1995) and abstract by R.X. Ynzunza et al. in the Advanced Light Source Compendium of Users Abstracts and Technical Reports, 1993-1996, Report LBNL-39981, UC411, p. 332.

[11] P.H. Citrin, G.K. Wertheim, and Y. Baer, Phys. Rev. Lett. 41, 1425 (1978); Phys. Rev. B 27, 3160 (1983).

[12] P.J. Feibelman and D.R. Hamann, Solid State Commun. 31, 413 (1979); Phys. Rev. B 20, 1433 (1979).

[13] B. Johansson and N. Martensson, Phys. Rev. B 21, 4427 (1980); Helv. Phys. Acta 56,405 (1983).

[14] C.S. Fadley, Prog. in Surf. Sci. 16, 275 (1984). 
[15] M.P. Seah and W.A. Dench, Surf. \& Interf. Anal. 1, 2 (1979).

[16] C.J. Powell, A. Jablonski, S. Tanuma, and D.R. Penn, J. Electron Spectrosc. 68, 605 (1994).

[17] (a) Y. Chen, H. Wu, Z. Hussain, and D.A. Shirley, to be submitted; (b) Y. Chen, F.J. García de Abajo, A. Chassé, R.X. Ynzunza, A.P. Kaduwela, M.A. Van Hove, and C.S. Fadley, submitted to Phys. Rev. B.

[18] (a) J.J. Rehr and R.C. Albers, Phys. Rev. B, 41, 81139 (1990); (b) A.P. Kaduwela, D.J. Friedman, and C.S. Fadley, J. Elec. Spectrosc. and Relat. Phenom. 57, 223 (1991): MCSD multiple scattering program package. available at http://electron.lbl.gov/mcsdpak/mcsdpak.html.

[19] M. Fink, A.C. Yates, Atomic Data, 1, 385 (1970).

[20] R.X. Ynzunza, Ph.D. thesis, University of California-Davis (1998), Appendix A.

[21] (a) M.A. Van Hove, S.Y. Tong, and M.H. Elconin, Surf. Sci., 64, 85 (1977); (b) R.S. Saiki, A.P. Kaduwela, M. Sagurton, J. Osterwalder, D.J. Friedman, and C.S. Fadley, Surf. Sci., 282, 33 (1993).

[22] (a) S. Andersson and J.B. Pendry, Sol. St. Commun., 16, 563 (1975); (b) M.A. Van Hove, W.H. Weinberg, and C.-M. Chan in "Low Energy Electron Diffraction, Experiment, Theory and Surface Structure Determination" (SpringerVerlag, Berlin 1986), Vol. 6, p. 246. 
[23] E.D. Tober, R.X. Ynzunza, F.J. Palomares, Z. Wang, Z. Hussain, M.A. Van Hove, and C.S. Fadley, Phys. Rev. Lett., 79, 2085 (1997). 

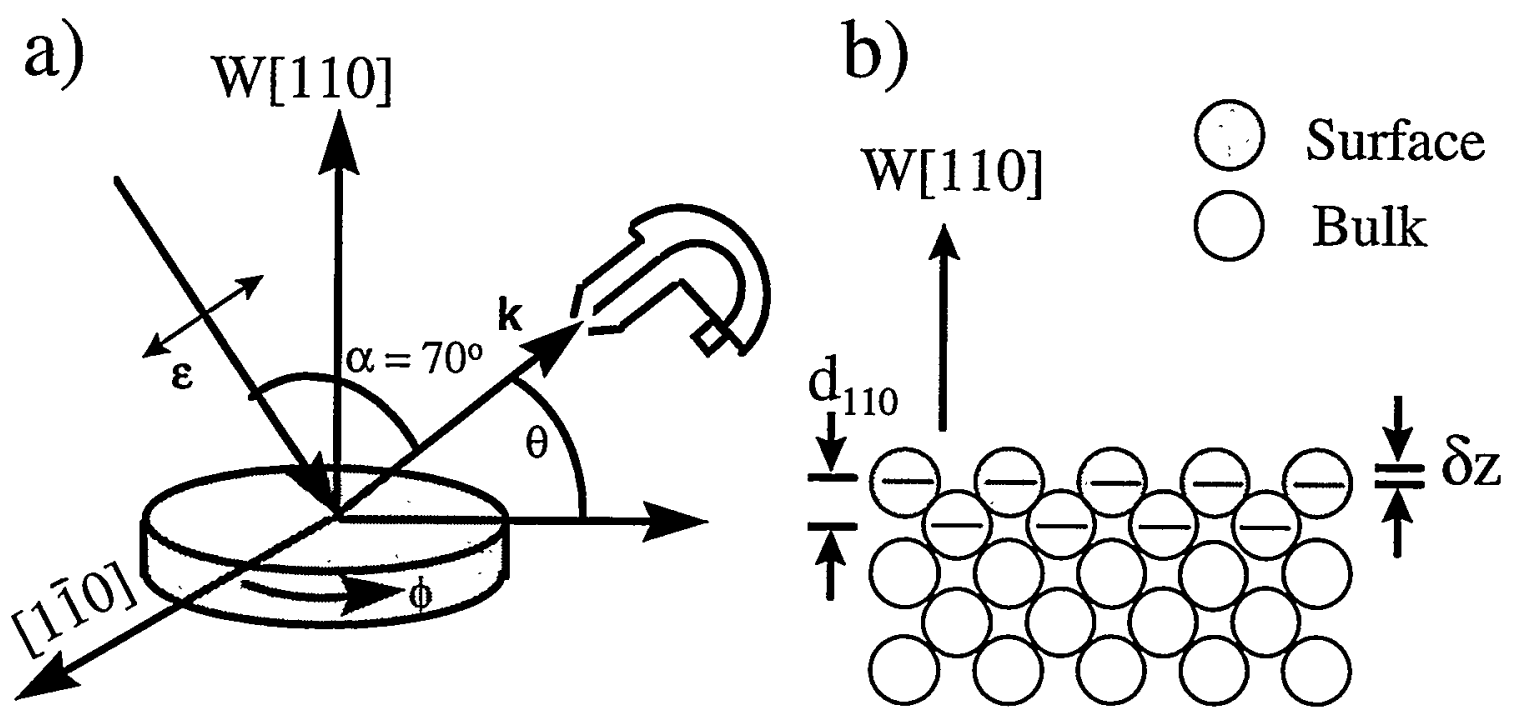

c)

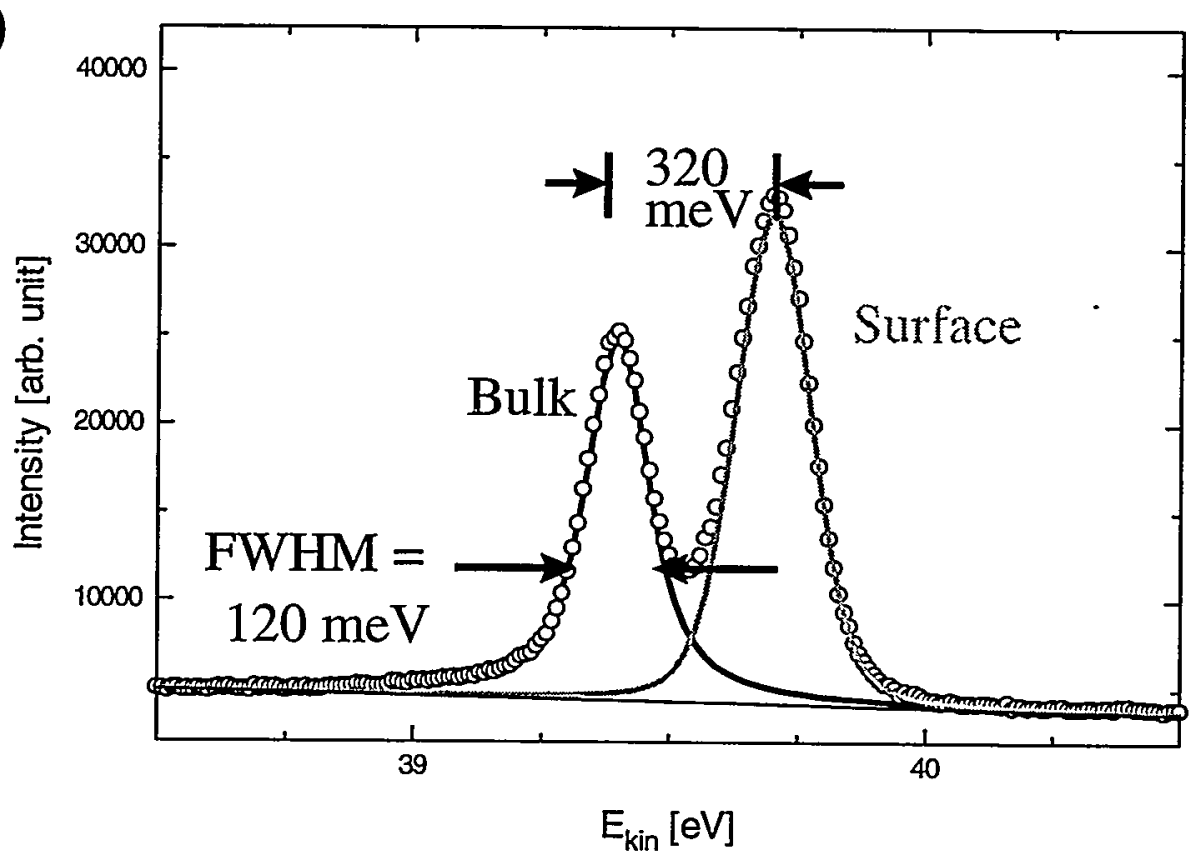

Figure 3.1. (a) The experimental geometry. (b) The atomic geometry, showing the two types of tungsten atom sites studied and the key structural parameters. (c) A W $4 \mathrm{f}_{7 / 2}$ photoelectron spectrum, showing the two component due to bulk atoms and surface atoms, with a surface core level shift (SCLS) between the two of $320 \mathrm{meV}$. Emission was at a takeoff angle with respect to the surface of $\theta=45^{\circ}$ and an azimuthal angle of $\phi=90^{\circ}$ (lying along the [100] direction in the W(110) surface). 


\section{Bulk}
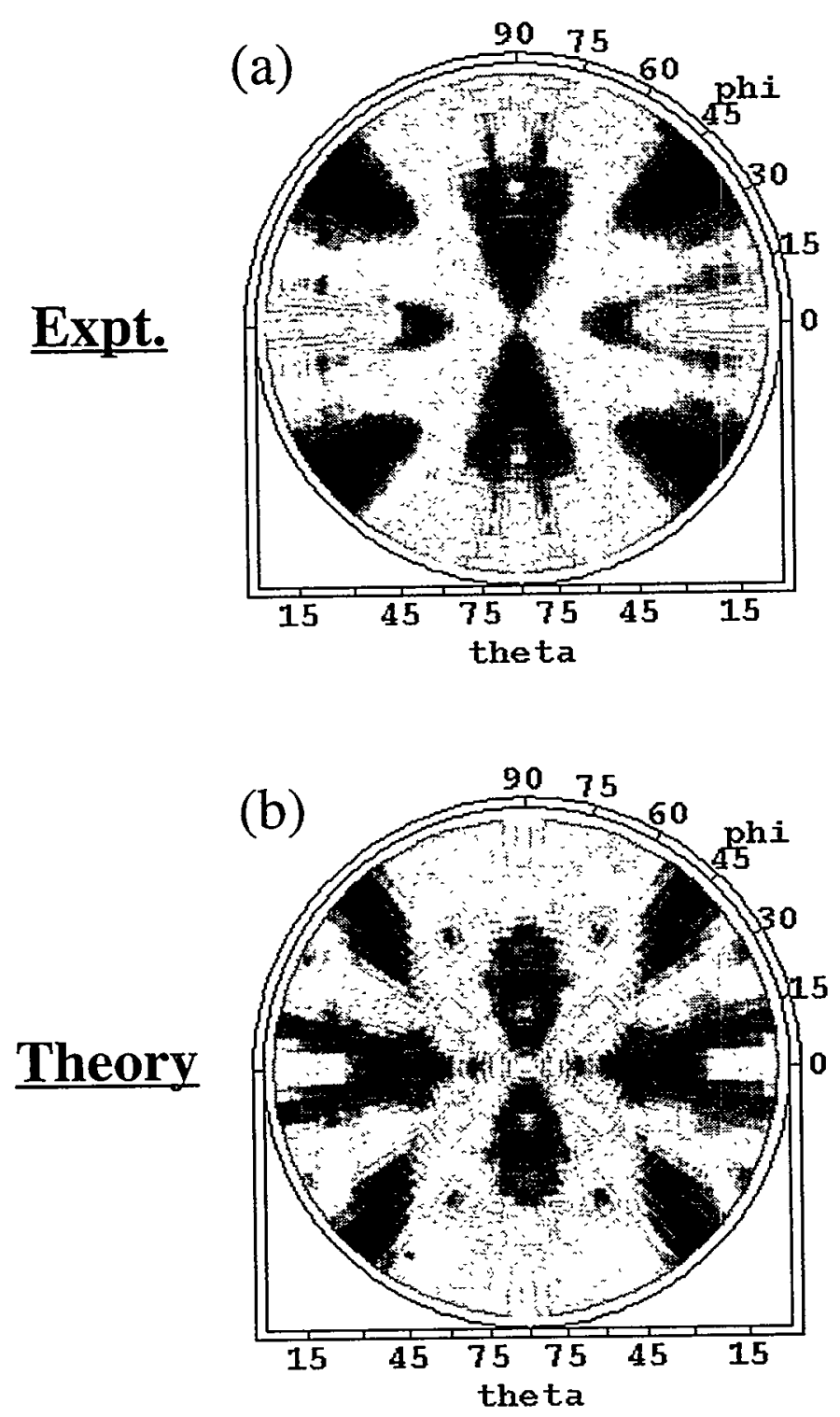

\section{$\underline{\text { Surface }}$}
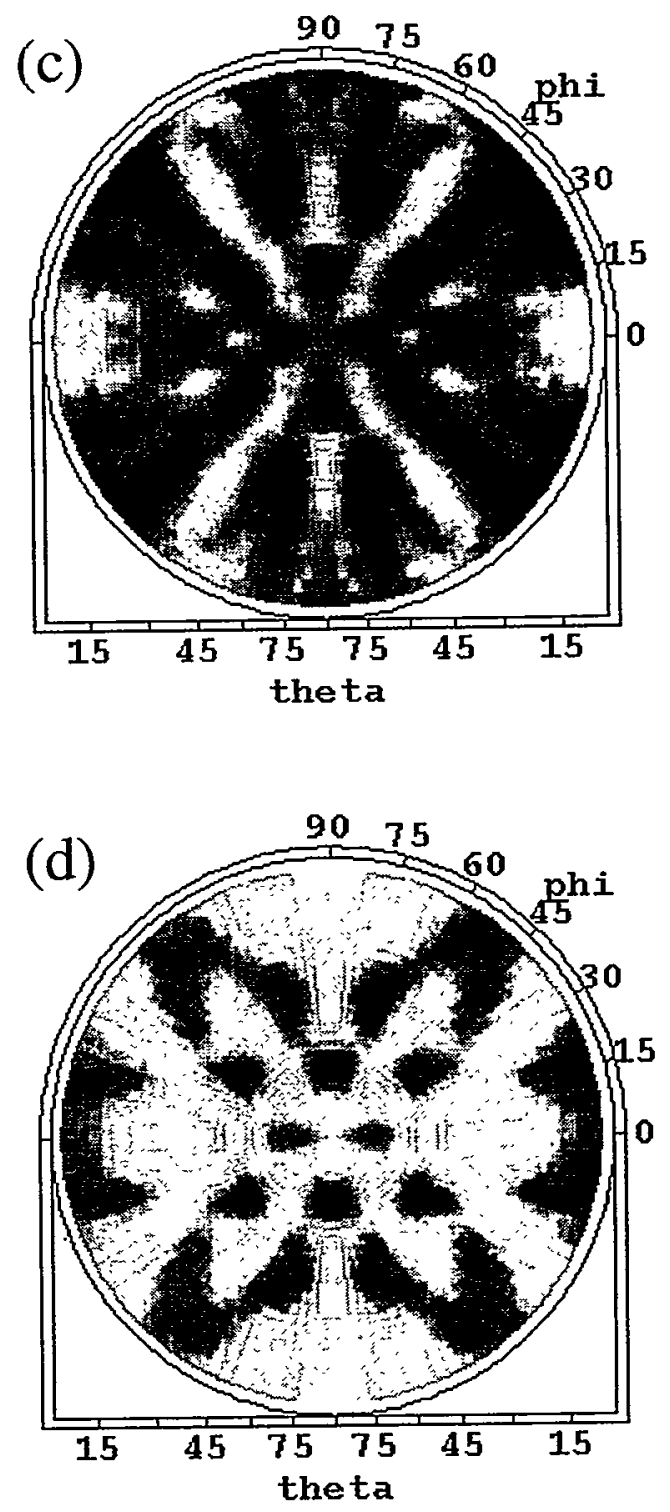

Figure 3.2. Full-solid-angle $\mathrm{W}_{4} \mathrm{f}_{7 / 2}$ photoelectron diffraction patterns: (a) bulk experiment, (b) bulk theory for the optimum interlayer distance, (c) surface experiment, and (d) surface theory for the optimum interlayer distance. 


\section{W(110) Attenuation Length: \\ $\mathrm{E}_{\mathrm{kin}}=40 \mathrm{eV}$}

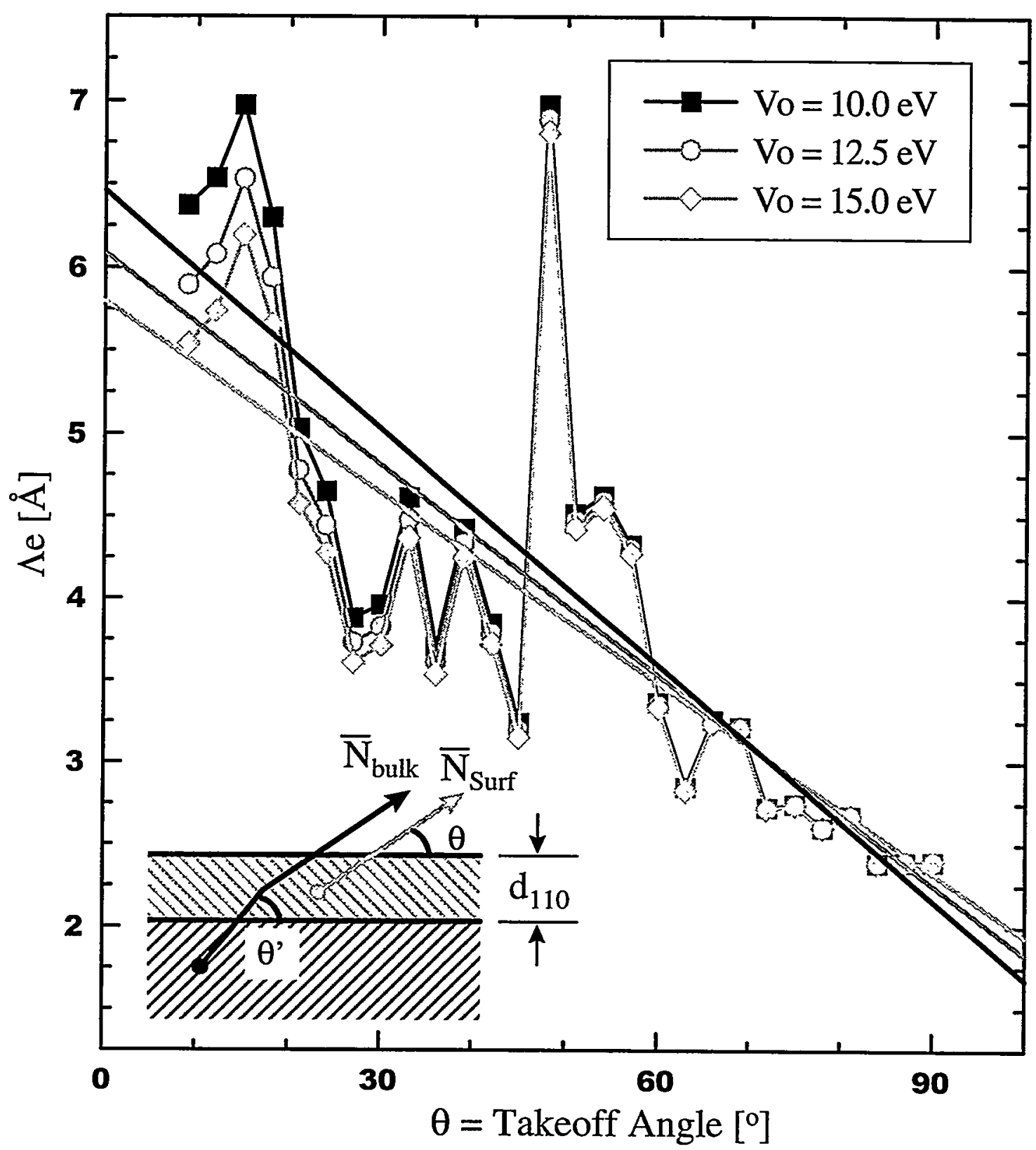

Figure 3.3. Determination of the effective electron inelastic attenuation length $\Lambda \mathrm{e}$ from the ratio of surface and bulk intensities via Eq. 3.1. The experimental intensities have been averaged in azimuth before taking the ratio, but some residual diffraction modulation of the curves is still evident, particularly at a takeoff angle of $\theta=47^{\circ}$. Calculations have been performed for three different values of the inner potential Vo, with $12.5 \mathrm{eV}$ representing the best value as judged by the structure analysis. 


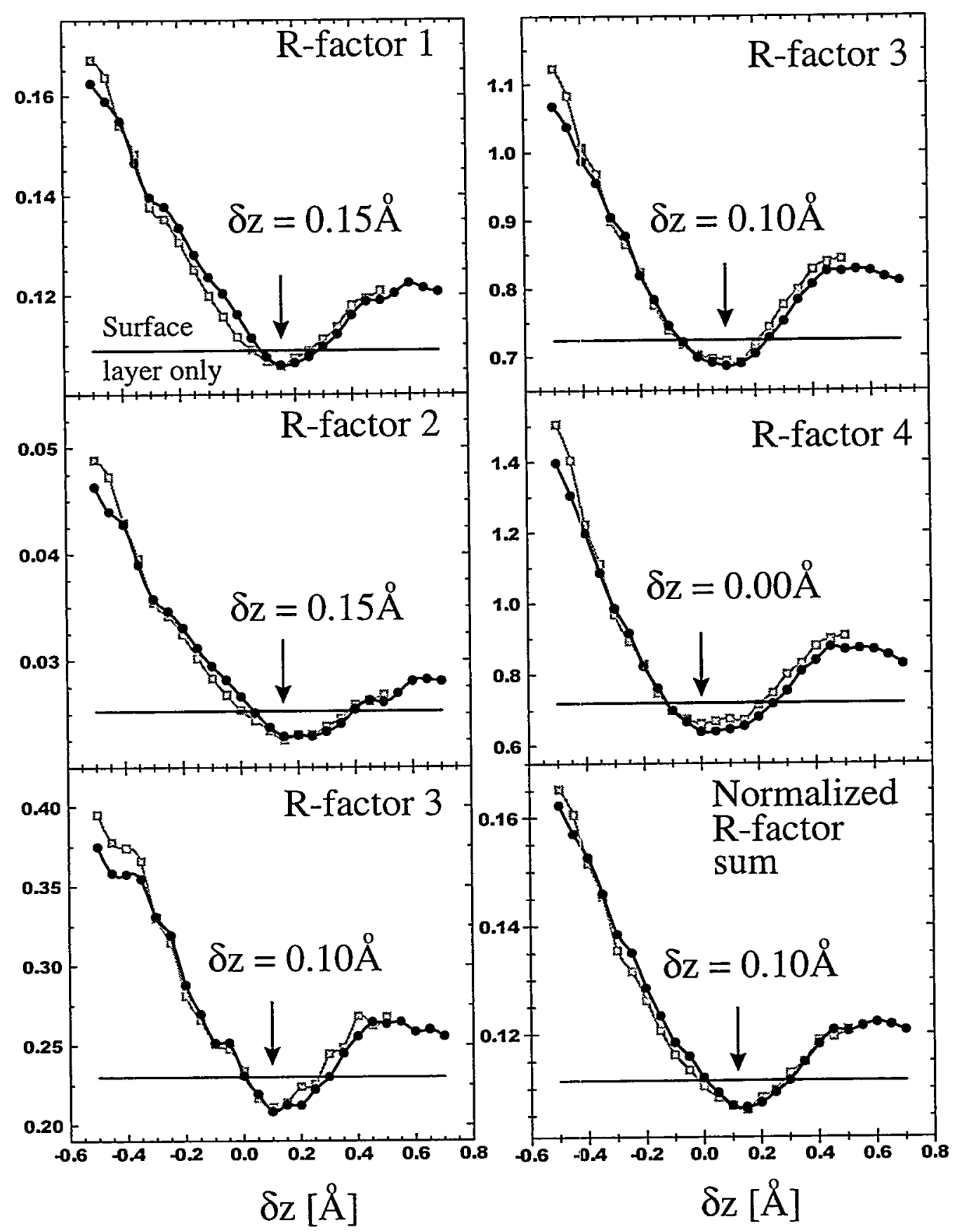

Figure 3.4. R-factor comparison of experiment and theory as a function of the interlayer relaxation $(\delta z)$ relative to the bulk separation $d_{110}$. Gray curves correspond to theoretical values calculated with our muffin-tin-potential derived phase shifts. Black curves correspond to theoretical values calculated with phase shifts from a prior LEED study by Heinz et al. [5]. 

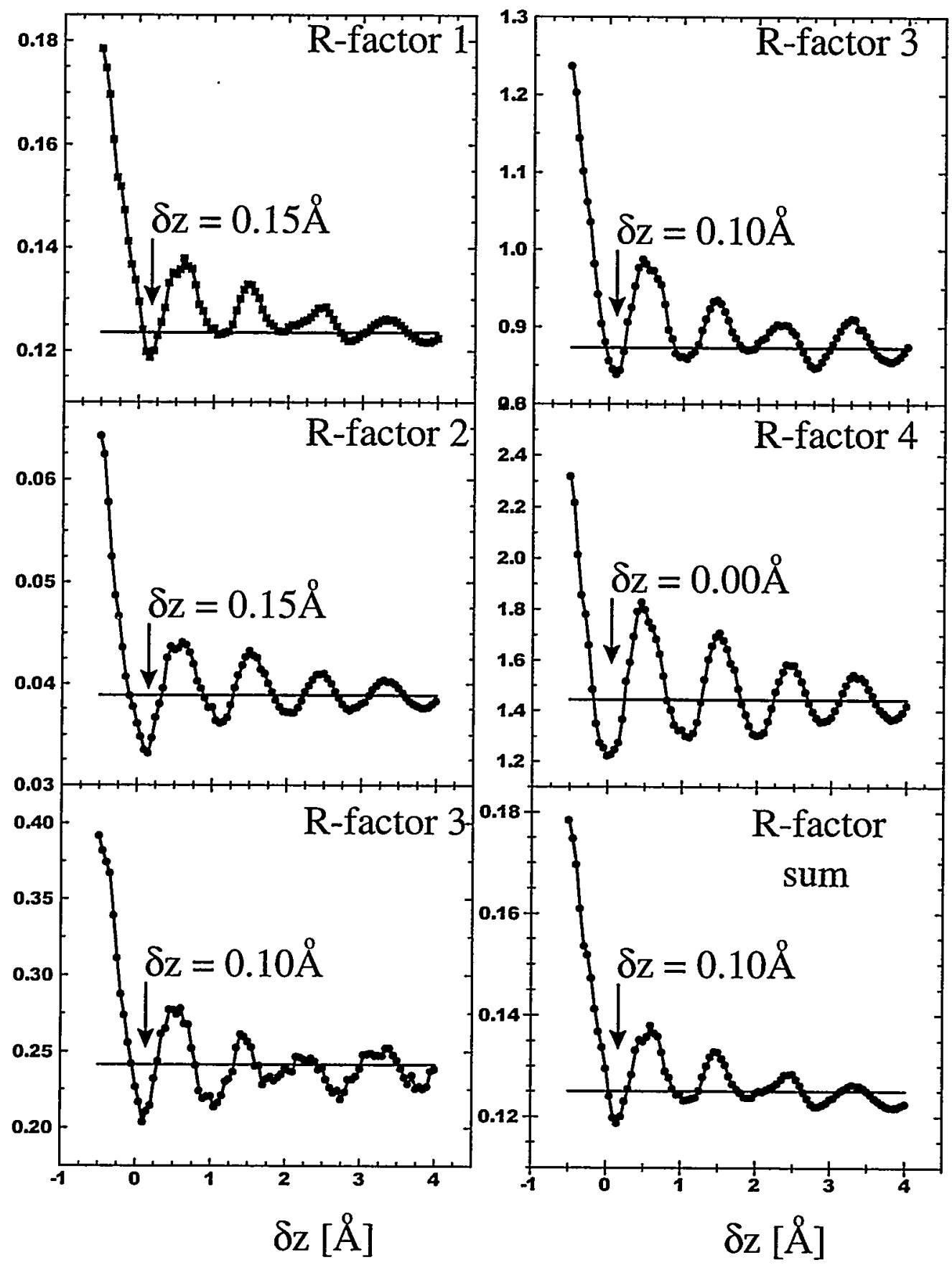

Figure 3.5. As Fig. 3.4 but with a much larger range in $\delta z$. Phase shifts were here taken only from a prior LEED study [5]. 


\title{
Chapter 4
}

\author{
Structure Determination for Saturated (1x1) Oxygen on W(110) from \\ Full-Solid-Angle Photoelectron Diffraction with Chemical-State Resolution
}

\section{I. INTRODUCTION}

The saturated one-monolayer adsorption of oxygen on W(110) has been studied previously by a number of other methods such as low energy electron diffraction (LEED), Auger electron spectroscopy, thermal desorption spectroscopy, work function measurements, and scanning tunneling microscopy (STM) [1-4], but several questions remain as to the precise adsorption structure involved. Earlier measurements concluded that superstructure spots seen in LEED, which were described as $c(14 \times 7)$ in symmetry, were due to a compressed misfit overlayer [2,3]. Later STM measurements made by Johnson et al. [4] found a local (1x1) symmetry and suggested that the oxygen atoms actually sit in the pseudo-threefold hollow sites of the (110) surface, as shown in Fig. 4.1(a). This is the same site thought to be occupied in the $\mathrm{p}(2 \times 1)$ structure formed at 0.5 monolayer (ML) [5], as well as other adsorption structures of oxygen on W(110) [3]. Also, as part of the STM study [4], the superstructure spots in the LEED pattern were found to be due to two types of striped (1x1) domains related by a site-exchange of two equivalent threefold-hollow adsorption sites on the surface and spaced at regular intervals of about $33 \AA$ and approximately (1x12) periodicity along the $[1 \overline{1} 3]$ and [ $[\overline{1} 13]$ directions (cf. Fig. 1 of ref. 4). In a more recent LEED and x-ray photoelectron diffraction (XPD) study, Daimon et al. [6(a)] have found this periodicity to be (1x12) (or less likely (1x13)), 
and that the striped domains align along the $[1 \overline{1} 2]$ and $[\overline{1} 12]$ directions (or about $10^{\circ}$ in azimuth away from the $[1 \overline{1} 3]$ and $[\overline{1} 13]$ directions seen previously [4]). Furthermore, the XPD data permitted estimating of the actual position of the oxygen in the threefold site from the positions of forward scattering maxima. The most likely atomic geometry of what we will here term the ( $1 \times 1) \times 12$ structure with an ideal site-exchange boundary between two domains is thus shown in Fig. 4.1(b). However, the large unit cell involved in this structure has prevented determining the actual oxygen position in this threefold site via LEED, and it is still unclear as to why it chooses to have the (1x1)x12 periodicity on our surface, or other periodicities in prior studies.

In order to better understand this interesting "incipient-oxide" structure, we have measured chemical-state-resolved low-energy photoelectron diffraction patterns for the $\mathrm{W}$ $4 f_{7 / 2}$ level, with these data permitting the first quantitative structure determination, including the two structural parameters $\mathrm{z}$ and $\ell$ defined in Fig. 4.1(c). Scanned-angle photoelectron diffraction (PD) data were obtained over nearly the full $2 \pi$ solid angle above the surface, with the $\mathrm{W}_{7 / 2}$ peak exhibiting a cleanly-shifted incipient-oxide peak that is displaced by about $+0.73 \mathrm{eV}$ from that due to bulk atoms, as illustrated in Fig. 4.2(a). This oxide peak more precisely corresponds to surface $\mathrm{W}$ atoms that are directly bound to three oxygen atoms in a local geometry that is $(1 \times 1)$ in character, and it is seen in high-resolution $\mathrm{W}$ 4f spectra from $\mathrm{O} / \mathrm{W}(110)$ well before reaching the saturated coverage $[6(b), 6(c)]$. The oxide and bulk diffraction patterns were measured separately, 
and the experimental data compared to multiple scattering theoretical simulations via $\mathrm{R}$ factors to determine the final structure.

\section{II. EXPERIMENTAL METHODOLOGY}

These experiments were performed on bend-magnet beamline 9.3.2 of the Advanced Light Source (ALS) at the Lawrence Berkeley National Laboratory [7(a)] using its advanced photoelectron spectrometer/diffractometer (APSD) experimental station [7(b)]. This facility has both a high resolution spherical grating monochromator and a high-resolution/high-throughput Scienta SES 200 spectrometer. The W(110) sample was prepared by an initial oxygen treatment at a temperature of $1200^{\circ} \mathrm{C}$ and a pressure of $1 \times 10^{-7}$ Torr $\mathrm{O}_{2}$ to reduce the amount of carbon from the near-surface region. This was followed by repeated flashing at up to $2200^{\circ} \mathrm{C}$ by electron bombardment heating to yield a clean surface as judged by both in situ $\mathrm{x}$-ray photoelectron spectroscopy (XPS) and a sharp (1x1) LEED pattern. The final oxide layer was formed via a separate surface reaction by exposing the sample to a pressure of $1.3 \times 10^{-6}$ Torr of oxygen at a temperature of $700^{\circ} \mathrm{C}$ for about 11 minutes or more, a recipe used in prior STM [4] and x-ray photoelectron diffraction studies [6] and leading for our particular surface to a sharp LEED pattern with (1x12) superstructures superimposed on the (1x1). Such LEED patterns obtained with incident energies of $85 \mathrm{eV}$ and $95 \mathrm{eV}$ are shown in Fig. 4.3; with (a) representing a single-domain structure and (b) a two-domain structure. Both of these patterns clearly show the primary $(1 \times 1)$ spots, plus the modulation by higher order spots trailing off into the $[1 \overline{1} \overline{1}]$ and the $[1 \overline{1} 1]$ directions. Although two domains of the (1x12) 
structure are expected with equal probability for a perfectly flat surface, we sometimes found only one domain to be dominant over much of the surface by scanning the LEED beam over it (as shown in Fig. 4.3(a)); this may be due to a slight tilt in the surface relative to (110), although we estimate the degree of miscut of the surface to be less than 0.5 degrees. It was previously determined by STM that these superstructure spots are due to the periodicity of domain walls oriented in the $[1 \overline{1} 3]$ and $[\overline{1} 13]$ direction and spaced about $26 \AA$ apart or 12-13 unit cells apart. With our preparation, domain walls formed in the $[1 \overline{1} 2]$ and $[\overline{1} 12]$ directions, giving us a $(1[1 \overline{1} 2] \times 12[1 \overline{1} \overline{1}])$ or more concisely (1x1)x12 pattern. With slight post annealing, a c(21x7) LEED pattern is observed that is more consistent with some prior observations on this surface [1], probably due to the desorption of some oxygen atoms. This structure at slightly less than $1 \mathrm{ML}$ coverage is consistent with the directions of domain walls seen in the STM work, and we thus suggest that some desorption could have occurred in this study as well [4].

To further quantify the issue of surface stoichiometry, we have checked the final coverage reached with our surface and preparation recipe for the $(1 \times 1) \times 12$ structure via a quantitative analysis of full-solid-angle $\mathrm{O}$ 1s and $\mathrm{W}$ 4f XPD data sets acquired at higher energies using a standard $\mathrm{Al} \mathrm{K \alpha} \mathrm{x}$-ray source. We quantitatively determined the coverage by examining the dependence of the $\mathrm{O} 1 \mathrm{~s} / \mathrm{W}$ 4f intensity ratio over the full solid angle diffraction pattern. The details of his analysis appear elsewhere [8], but from the measured $\mathrm{O} 1 \mathrm{~s} / \mathrm{W} 4 \mathrm{f}$ ratio, we have used standard XPS quantitative analysis formulas and photoelectric cross sections [9] to determine that there are $1.04 \pm 0.1$ monolayers (ML's) 
of oxygen at saturation, in excellent agreement with the coverage expected for the saturated surface. The base pressure in the chamber where the PD measurements were made was $1.3 \times 10^{-10}$ Torr. Due to the passivation of the surface by the oxidation, this oxygen monolayer was quite inert and allowed measurements for several days without any measurable buildup of contaminants or changes in the structure, as verified via both XPS the lack of qualitative changes in the LEED pattern.

The experimental geometry is shown in Fig. 4.2(b). The angle $\alpha$ between the linearly-polarized incident radiation and the electron emission direction was kept fixed at $70^{\circ}$, with the polarization vector $\varepsilon$ lying in the plane of the [110] surface normal and the photoelectron wave vector $\mathbf{k}$; the light was thus p-polarized in the usual notation. The sample was rotated on two perpendicular axes using a custom-built goniometer, with the polar angle $\theta$ being measured relative to the surface (i.e. as a takeoff angle) and the azimuthal angle $\phi$ measured relative to the $[1 \overline{1} 0]$ direction, so as to obtain the full diffraction pattern. The excitation energy used was $170 \mathrm{eV}$ (obtained as fourth-order of $42.5 \mathrm{eV}$ from a spherical-grating monochromator), resulting in photoelectrons with a kinetic energy of approximately $140 \mathrm{eV}$.

We measured $\sim 900 \mathrm{~W} 4 f_{7 / 2}$ spectra over nearly the full $2 \pi$ solid angle above the surface, which spanned the takeoff angle range from $\theta=9^{\circ}$ to $90^{\circ}$ (= normal emission) in $3^{\circ}$ steps and the $180^{\circ}$ azimuthal angle range from $[1 \overline{1} 0]$ to $[\overline{1} 10]$. The angle step for each azimuthal scan was determined according to the equation: $\Delta \phi(\theta)=$ $\left[\sin \left(\theta_{\mathrm{i}} / 2\right) / \sin (\theta / 2)\right] \Delta \phi_{\mathrm{i}}$, with $\theta_{\mathrm{i}}=9^{\circ}$ and $\Delta \phi_{\mathrm{i}}=3^{\circ}$; in this way, the azimuthal step size was 
adjusted with takeoff angle such that the final data set consisted of spectra representing roughly equal solid-angle increments. Angles below $9^{\circ}$ were not used due to the much lower count rates and thus longer counting times involved. The analyzer acceptance angle is approximately a $\pm 5^{\circ}$ cone. The monochromator exit slit was set to 30 microns width and the analyzer pass energy to $20 \mathrm{eV}$, with these choices leading to an overall instrumental contribution to the resolution of approximately $50 \mathrm{meV}$. The resulting intensities permitted taking a high-resolution spectrum such as that in Fig. 4.2(a) in about 20 seconds; the actual data acquisition for all spectra thus took about 5 hours, or slightly more than one fill duration of the ALS storage ring. Due to the symmetry of the crystal and the oxygen overlayer, only a $90^{\circ}$ azimuthal scan is in principle needed to provide all the information for a full $360^{\circ}$ azimuthal scan. However, in order to increase accuracy and average over any small sample misalignments, a $180^{\circ}$ range in $\phi$ was measured and this data was then mirror-averaged into $90^{\circ}$ and finally repeated four times so as to obtain the full $360^{\circ}$ azimuthal pattern. As Fig. 4.2(a) indicates, the spectra were easily resolved into oxide and bulk components by fitting them with analytical functions, in particular asymmetric Voigt functions, of at first variable width and spacing, riding on a Shirleytype inelastic background. In the final fits, the widths and spacings were kept fixed at their average values over each azimuthal scan to minimize spurious intensity changes. The bulk peak is found to occur at a kinetic energy of $140.2 \mathrm{eV}$ and corresponds to the multilayer signal from all the tungsten atoms below the top layer of tungsten. The chemically-shifted peak has a higher binding energy by $+730 \mathrm{meV}$, and this has been verified to be due to a strongly bound "incipient-oxide-like" species associated with the top layer of tungsten atoms that are directly bonded to three oxygen atoms (cf. Figs. 4.1(a) 
and 4.1(b)) [6(b),6(c)]. These two intensities thus permit deriving site-specific patterns from both the top layer of tungsten atoms which form the interface layer and the tungsten atoms below this interface layer. Any variations of radiation flux with time were normalized out in determining the final intensities $I(\theta, \phi)$. These two intensities were then normalized into a chi function $\chi(\theta, \phi)=\left[I(\theta, \phi)-I_{0}(\theta)\right] / /_{0}(\theta)$, with $I_{0}(\theta)$ being the estimated intensity in the absence of diffraction effects. $I_{0}(\theta)$ was determined by first averaging $I(\theta$, $\phi)$ over each $\phi$ value to produce $\bar{I}(\theta)$, and then fitting $\bar{I}(\theta)$ with a smooth low-order spline function in $\theta$ to finally yield $\mathrm{I}_{0}(\theta)$. Figs. 4.4(a) and 4.4(c) show the final separate experimental diffraction pafterns $\chi_{\text {bulk }}$ and $\chi_{\text {oxide, }}$, respectively. Not surprisingly, the two patterns are quite different. We have only analyzed the oxide pattern in determining the adsorption structure, as this layer of $\mathrm{W}$ atoms should be the most sensitive to the position of the overlying oxygen monolayer.

\section{III. STRUCTURE ANALYSIS AND DISCUSSION}

To determine the structure of the $\mathrm{O} / \mathrm{W}(110)(1 \times 1) \times 12$ surface, comparisons were made between experiment and theoretical calculations performed with a program developed by Y. Chen et al. [10] and based on the Rehr-Albers separable-Green's function formulation [11]. The several non-structural inputs needed for these calculations are as follows: Due to a change in the work function of the sample as one monolayer of oxygen is adsorbed, the inner potential is estimated to change from $12.5 \mathrm{eV}$ for a clean $\mathrm{W}(110)$ surface [8] to $11.0 \mathrm{eV}$ for the $\mathrm{O} / \mathrm{W}(110)(1 \times 1) \times 12$ surface [3]. The electron inelastic attenuation length $\Lambda_{e}$ was estimated from an experimental value at lower energy 
as extrapolated to higher energy using the relation $\Lambda_{e}=C E^{1 / 2}$, where $C$ is an empirical constant and $\mathrm{E}$ is the electron kinetic energy. This formula was derived by Seah and Dench using experimental attenuation lengths for several solid elements [13]. The parameter $\mathrm{C}$ was determined from an experimental determination for the clean W(110) surface of $3.0 \AA$ at a kinetic energy $\mathrm{E}$ of $40 \mathrm{eV}$, as shown elsewhere [8]. The final value for $\Lambda_{\mathrm{e}}$ at our energy was thus $5.0 \AA$. To describe vibrational effects, the calculations include Debye-Waller factors based on uncorrelated vibrational motion, with a Debye temperature of $400 \mathrm{~K}$ for tungsten and measurement at room temperature $(298 \mathrm{~K})$. The angular averaging in the program was set to $\pm 3^{\circ}$, although the actual experimental angular aperture was $\pm 5^{\circ}$. To allow for the possibility of too little angular averaging in the theory, the calculated $2 \pi$ solid angle diffraction patterns were finally convoluted with a twodimensional gaussian function in $\theta$ and $\phi$, with a $4^{\circ}$ full width at half maximum (FHWM) in amplitude that was found by R-factor tests to yield the best overall agreement with experiment. The atomic cluster used contained 100 atoms, including a full layer of 30 (1x1) $\mathrm{O}$ atoms and 4 layers of $\mathrm{W}$ underneath it. Theory was finally compared to experiment via a set of five $\mathrm{R}$ factors (R1-R5) that have been used previously in XPD, and these are discussed in detail elsewhere $[8,13]$. Corollary structural information to begin the structure search came as well from a similar analysis of an experimental

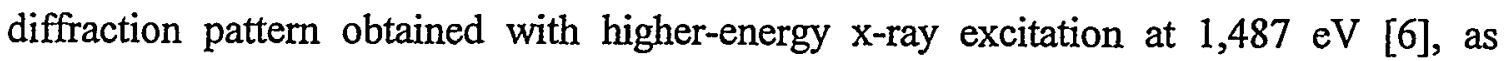
reproduced in Fig. 4.5(a). The high-energy XPD study showed from the forward scattering directions associated with higher $\mathrm{W} 4 \mathrm{f}$ photoelectron kinetic energies that the oxygen atom is laterally displaced along the $[1 \overline{1} 0]$ (or $[\overline{1} 10]$ depending on the siteexchanged domain type) direction from the center of the pseudo-threefold hollow site. 
As Fig. 4.1(c) illustrates, the exact position can thus be defined by specifying the distances $z=$ the vertical interlayer separation of the interface tungsten and the oxygen and $\ell=$ the lateral displacement of the oxygen overlayer from the tungsten substrate in the $<1 \overline{1} 0>$ directions, with $\ell=1.67 \AA$ corresponding to three equal bond distances between the overlying oxygen atoms and tungsten. The XPD study suggests via $\mathrm{R}$-factor analysis that $\mathrm{z} \approx 0.84 \AA$ and that a deviation from the equal-bond-distance case exists, with $\ell \approx$ $1.52 \AA$.

Lower-energy scanned-angle PD data is expected to be more sensitive to interplanar distances and local structure due to the greater importance of back and side scattering [14]. To determine the final optimum atomic positions for the oxygen in this incipient oxide structure, we have thus used the structure derived from XPD as a starting point, and compared our low-energy experimental and theoretical PD data sets while varying both $\mathrm{z}$ and $\ell$ and monitoring all five $\mathrm{R}$-factors, as well as a normalized sum $\left(R_{\text {sum }}\right)$ of the five; the normalization was carried out by making the minimum value and the full excursion of each of the five $R$-factors over the full range of $\mathrm{z}$ and $\ell$ studied have the same values, and then carrying out a sum over all five [8]. The minimum in the normalized sum should thus represent the best overall estimate of the structure. A summary of these R-factor results is shown in Fig. 4.6, and it is clear that, with the exception of R2 (based on squared differences between experimental and theoretical chi functions), the R-factor minimum is found in very nearly the same place for all of the fundamental R-factors. Even R2 has a local minimum very close to the absolute minima of the other four. The normalized sum also yields the same minimum as R1, R3, R4, and 
R5, and we thus are finally able to conclude that the oxygen sits in a quasi-threefold hollow site on the $\mathrm{W}(110)$ surface, with an interlayer separation $\mathrm{z}$ from the first $\mathrm{W}$ layer of $0.91 \pm 0.05 \AA$ and a lateral displacement $\ell$ of $1.57 \pm 0.05 \AA$ from each $W$ atom in the [1 10$]$ (or $[\overline{1} 10]$ ) direction. The final theoretical diffraction pattern for the best fit geometry is shown in Fig. 4.4(b), and it is in general in excellent agreement with experiment. The final structure is also within our error limits of the XPD structure, although we expect the low-energy values derived here to be somewhat more accurate.

As noted above, if the oxygen atom were in the center of the pseudo-threefold hollow with the bond length to each of the three atoms being the same, this lateral displacement $\ell$ would be $1.67 \AA$. The value of $1.57 \AA$ thus leads to two different bond lengths between the oxygen and the tungsten: one of them is $1.79 \AA$ and lies along the [1 10$]$ (or [ $\overline{1} 10]$ ) direction, and the other is $1.94 \AA$ and is associated with the other two atoms that make up the threefold hollow with a rotation of $\pm 67^{\circ}$ away from the [1 $\left.\overline{1} 0\right]$ direction, as can be directly seen in the most prominent features in the XPD diffraction pattern in Fig. 4.5(a), as discussed in more detail elsewhere [6]. Figs. 4.5(a) and 4.5(b) further compare XPD experiment and theory for our optimized geometry, and there is again excellent agreement. Thus, both low-energy and high-energy PD data are found to agree very well concerning this structure. Furthermore, the W-O bond distances found here to be within the range of those reported in bulk W oxides of 1.46-2.94 $\AA$ [15].

We can finally compare our results to the only other structural determination completed to date for the $\mathrm{O} / \mathrm{W}(110)$ system, a LEED study of the stable $1 / 2 \mathrm{ML}$ coverage 
$\mathrm{p}(2 \times 1)$ overlayer in which the bond length is found to be $2.08 \AA$ between the oxygen and the tungsten. In the LEED determination, however, only the interlayer spacing was varied, and no allowance was made for the lateral displacement $\ell$. This corresponds to the oxygen layer having a height $\mathrm{z}$ of $1.24 \AA$ that is significantly larger than our value [5]. Our geometry for the $(1 \times 1) \times 12$ structure is thus significantly different from this $\mathrm{p}(2 \times 1)$ LEED result.

\section{IV. CONCLUSIONS}

The atomic geometry of a one-monolayer: saturated oxygen structure on $W(110)$, which we have designated (1x1) 12 , has thus been determined, and is found to correspond to a vertical interplanar distance of $\mathrm{z}=0.91 \pm 0.05 \AA$ and a lateral displacement of $\ell=1.57 \pm 0.05 \AA$ that leads to two different $\mathrm{O}$-W bond lengths. This study thus demonstrates the considerable potential of chemical-state resolved photoelectron spectroscopy at a third-generation synchrotron radiation source for studying adsorption by using photoelectron diffraction to determine the local atomic geometries around species in different bonding sites. This study also illustrates the ability of multiple scattering theory and R-factor analysis of such data to permit determining slight deviations from higher symmetry adsorption positions. While incident-beam diffraction-based techniques such as LEED can provide detailed structural information for systems with relatively small unit cells, photoelectron diffraction has the advantage of being a short-range-order probe capable of determining local average structure even in very large unit cells, as long as the vast majority of atoms within the unit cell have the same near-neighbor 
environment. With STM, it is possible to directly derive the atomic positions within the outermost exposed layers, but it is much more difficult to extract detailed information on the height of an overlayer above the underlying atoms ( $\mathrm{z}$ in the present case) or on the precise lateral registry of the overlayer ( $\ell$ in the present case). The complementarity of these three particular methods is thus evident. Various areas of application of chemicalstate resolved photoelectron diffraction are thus suggested by this work, including metal oxidation, metal-metal epitaxy, semiconductor surface reactions, and model studies of heterogeneous catalysis. 


\section{References:}

[1] L.H. Germer and J.W. May, Surf. Sci. 4, 452 (1966).

[2] (a) E. Bauer, Surf. Sci. 7, 351 (1967); (b) E. Bauer and T. Engel, Surf. Sci. 71, 695 (1978).

[3] C.R. Brundle and J.Q. Broughton, "The Initial Interaction of Oxygen with Welldefined Transition Metal Surfaces," appearing in The Chemical Physics of Solid Surfaces and Heterogeneous Catalysis, 3, p.131, D.A. King and D.P. Woodruff (Eds.), (Elsevier, 1990).

[4] K.E. Johnson, R.J. Wilson, and S. Chiang, Phys. Rev. Lett. 71, 1055 (1993).

[5] M.A. Van Hove and S.Y. Tong, Phys. Rev. Lett. 35, 1092 (1975).

[6] H. Daimon, R.X. Ynzunza, J. Palomares, H. Takagi, and C.S. Fadley, Surf. Sci. 408, 260 (1998); (b) R.X. Ynzunza et al., in the Advanced Light Source Compendium of Users Abstracts and Technical Reports, 1993-1996, Report LBNL-39981, UC-411, p. 398; (c) D. M. Riffe and G.K. Wertheim, Surf. Sci. 399, 248 (1998).

[7] (a) Z. Hussain, W. R. A. Huff, S. A. Keller, E. J. Moller, P. A. Heimann, W. McKinney, H. A. Padmore, C. S. Fadley, and D. A. Shirley, J. Elec. Spec. and Rel. Phen. 80, 401 (1996); (b) C.S. Fadley, M.A. Van Hove, Z. Hussain, and A.P. Kaduwela, J. Elec. Spec. and Rel. Phen. 75, 273 (1995). 
[8] R.X. Ynzunza, Ph.D. thesis, University of California-Davis (1998); Chapters 2 and 3.

[9] (a) C.S. Fadley, Prog. in Surf. Sci. 16, 275 (1984); (b) J.J. Yeh and I. Lindau, Atomic Data and Nuclear Data Tables 32, 1 (1985).

[10] (a) Y. Chen, H. Wu, Z. Hussain, and D.A. Shirley, unpublished results; (b) Y. Chen, F.J. García de Abajo, A. Chassé, R.X. Ynzunza, A.P. Kaduwela, M.A. Van Hove, and C.S. Fadley, Phys. Rev. B, in press; further details concerning this multiple scattering program are available at http://electron.lbl.gov/mscdpack/mscdpack.html.

[11] J.J Rehr and R.C. Albers, Phys. Rev. B, 41, 81139 (1990).

[12] M.P. Seah and W.A. Dench, Surf. \& Interf. Anal. 1, 2 (1979):

[13] R.S. Saiki, A.P. Kaduwela, M. Sagurton, J. Osterwalder, D.J. Friedman, C.S. Fadley, and C.R. Brundle, Surf. Sci. 282, 33 (1993).

[14] C. S. Fadley, in Synchrotron Radiation Research: Advances in Surface and Interface Science, R. Z. Bachrach, Ed. (Plenum Press, New York, 1992).

[15] Crystal Data Determinative Tables, Volume 4: Inorganic Compounds, Eds. H.M. Ondik and A.D. Mighell, (Published jointly by the U.S. Department of Commerce, National Bureau of Standards, and the JCPDS- International Centre for Diffraction Data, 1969); R.W.G. Wyckoff, in Crystal Structures, (Interscience Publishers, New York, (1963). 
a)

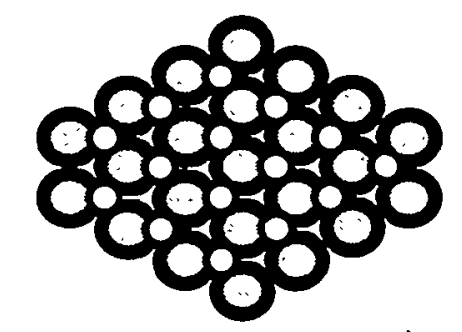

[001]

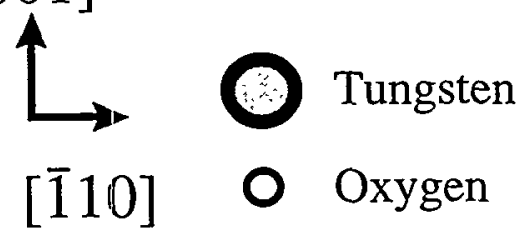

b)

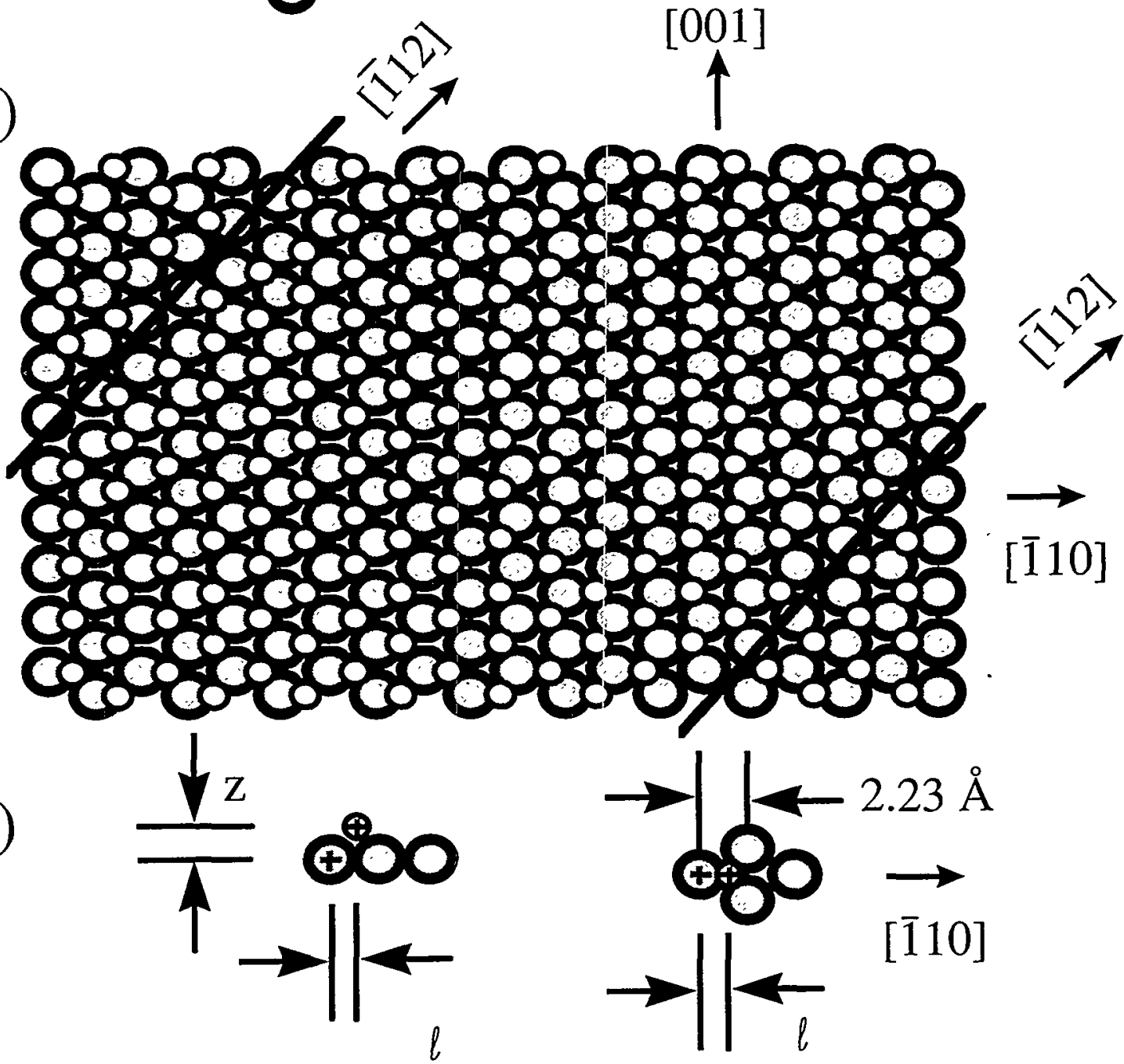

Figure 4.1. a) The top layer of tungsten with one monolayer of oxygen in the $\mathrm{O} / \mathrm{W}(110)(1 \mathrm{x} 1)$ structure and oxygen sitting in one of two possible three-fold hollow sites. Oxygen atoms are denoted by the white circles and tungsten atoms by the grey circles. b) The $(1 \times 1) \times 12$ structure studied here, with domain walls along the [112] direction. c) The nearest-neighbor geometry for an adsorbed oxygen atom. The parameters varied for the $\mathrm{R}$-factor analysis are the height of the oxygen layer above the tungsten surface $(z)$ and the lateral displacement of the oxygen as referenced from the ontop position $(\ell)$. 
a)

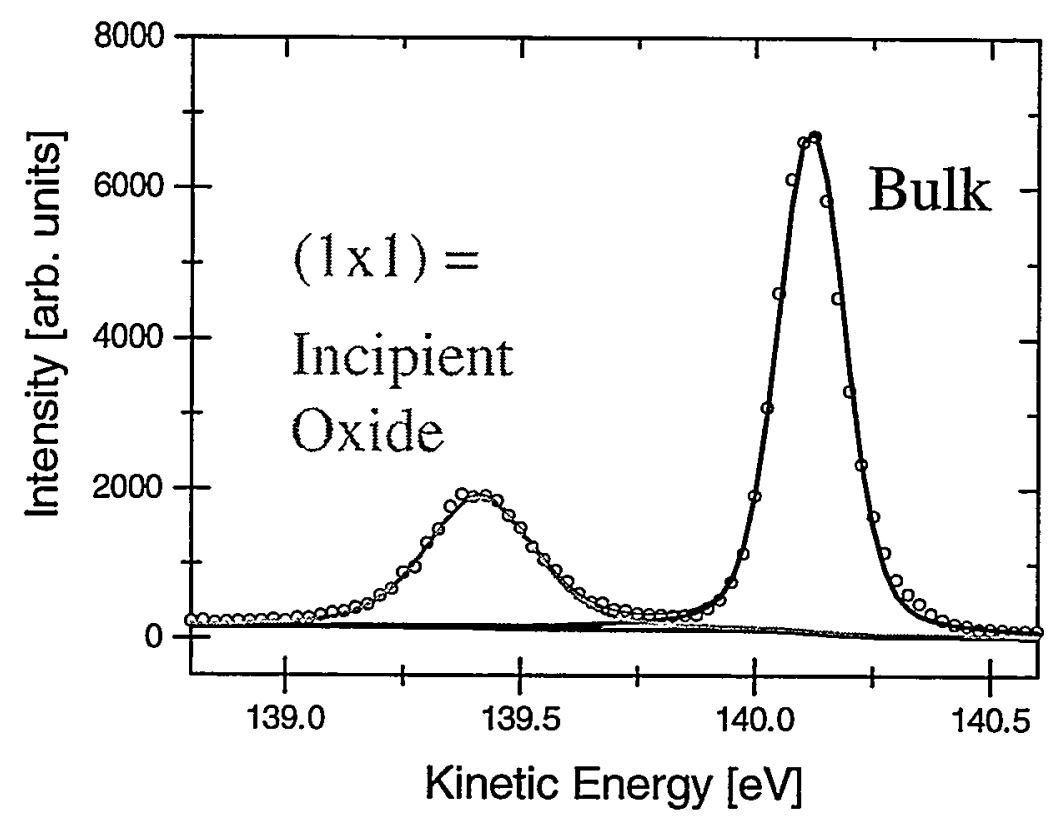

b)

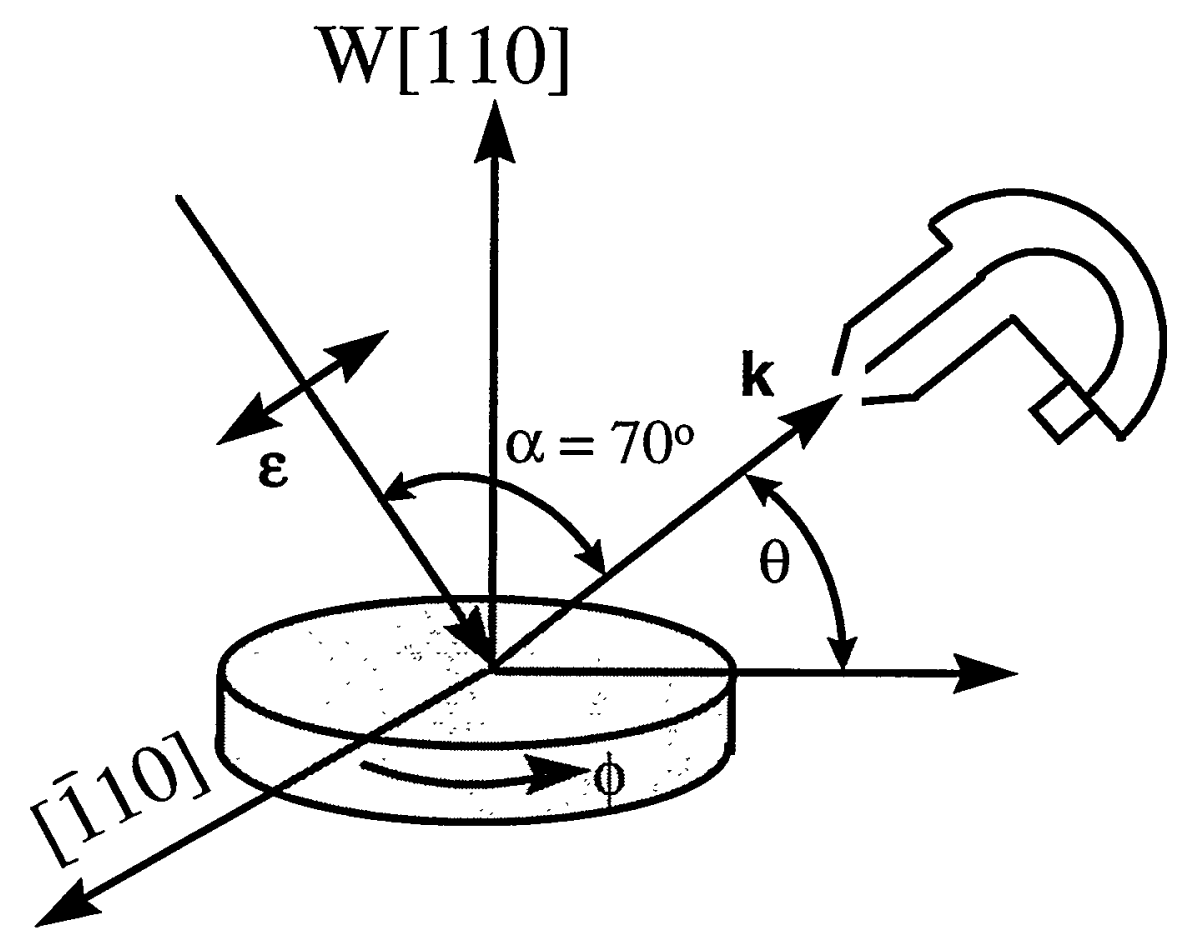

Figure 4.2. a) W $4 \mathrm{f}_{7 / 2}$ spectrum from the $(1 \times 1) \times 12$ structure with $\theta=45^{\circ}$ and $\phi=90^{\circ}$ or along the $<001>$ azimuthal direction. b) Experimental geometry. 
a)

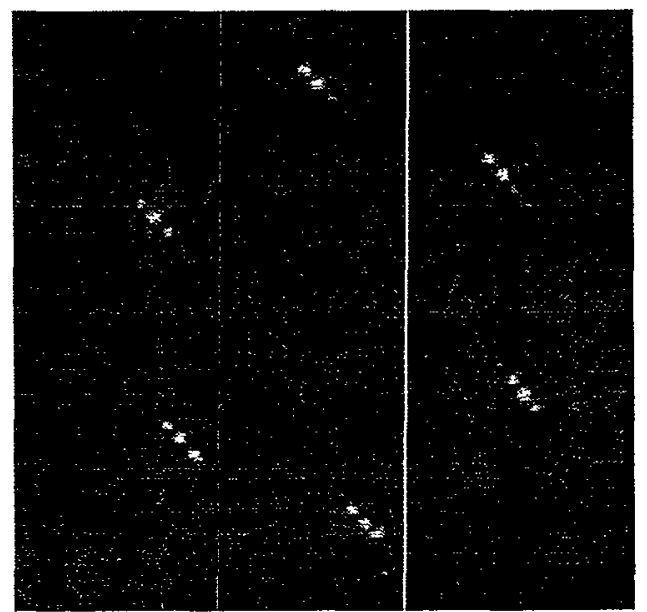

b)

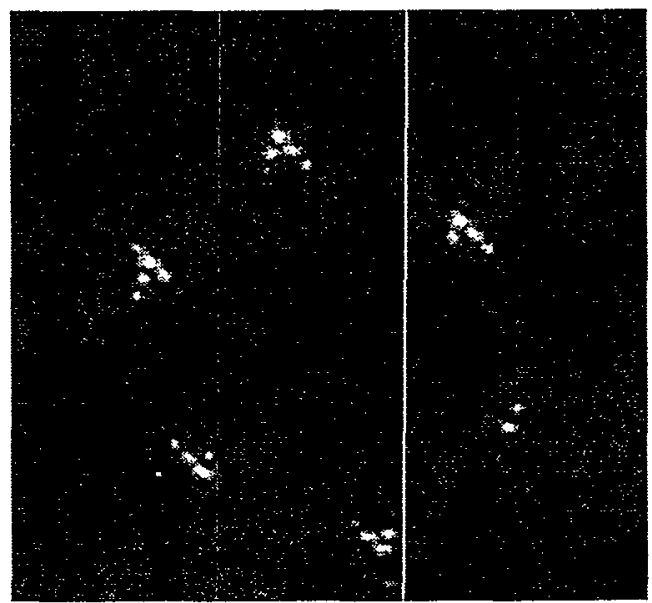

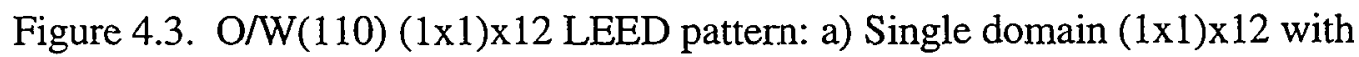
$85 \mathrm{eV}$ incident electron energy , b) two-domain (1x1) $\times 12$ with $95 \mathrm{eV}$ incident electron energy. 


\section{a) Oxide: Expt.}

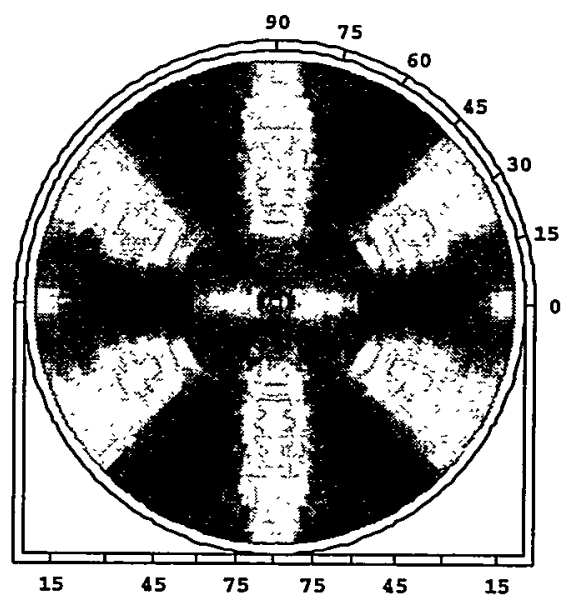

b) Oxide:
Theory

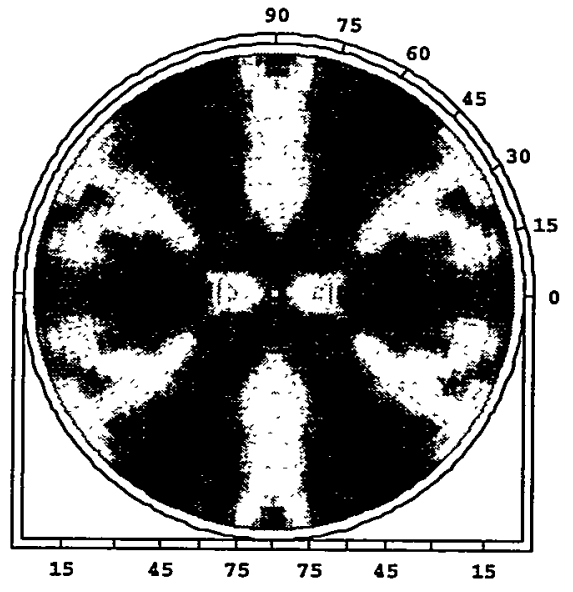

c) Bulk:
Expt.

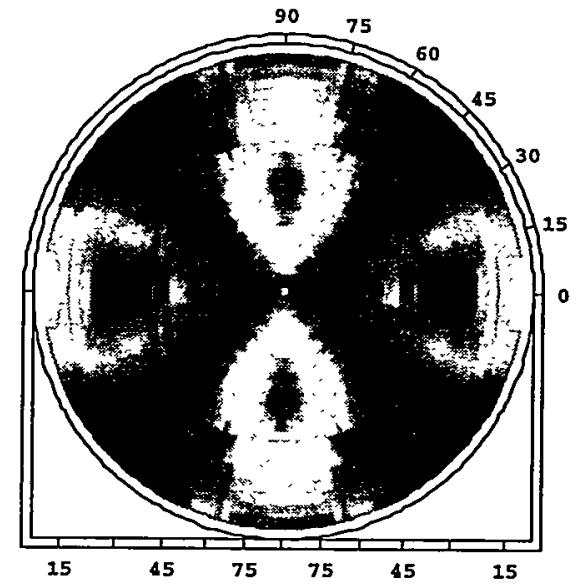

Figure 4.4. Full-solid angle $W 4 f$ photoelectron diffraction data $\left(E_{\text {kin }}=140 \mathrm{eV}\right)$ from

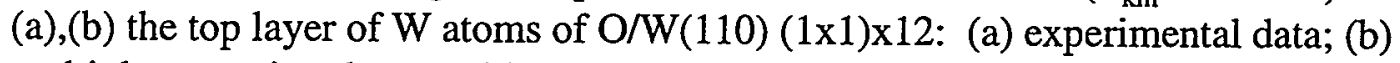
multiple scattering theory with oxygen atoms occupying the threefold hollow sites at the optimized structure parameters of $z=0.91 \AA$ and $l=1.57 \AA$ and (c) the underlying $\mathrm{W}$ bulk atoms, experimental data only. 


\section{a) Oxide: Expt.}

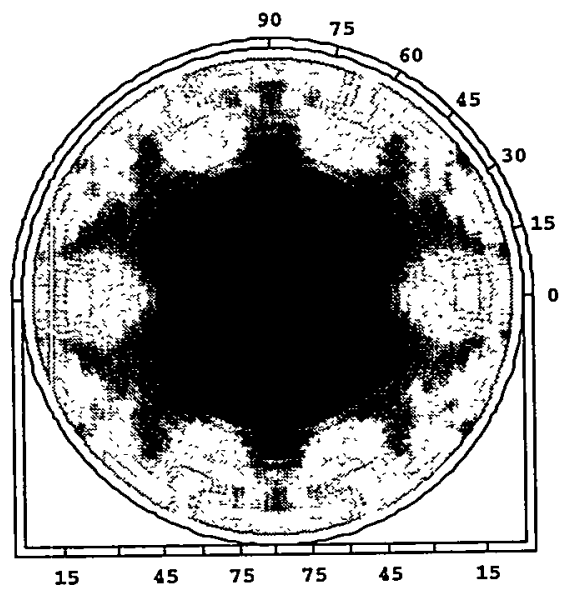

\section{b) Oxide: Theory}

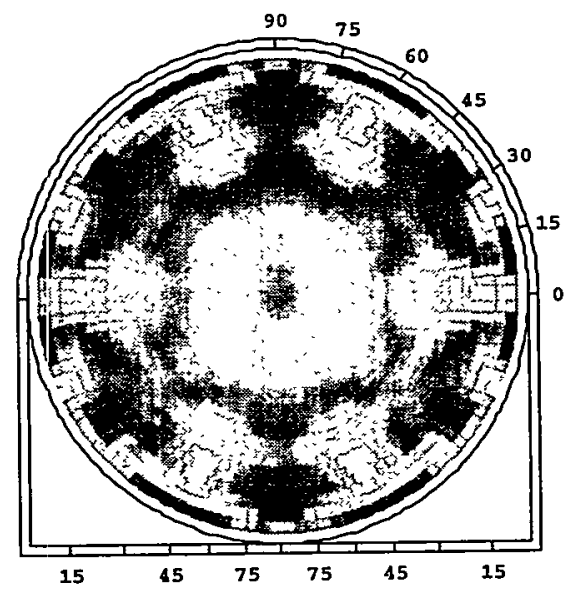

Figure 4.5. Full-solid angle $\mathrm{W} 4 \mathrm{f}$ high-energy photoelectron diffraction $\left(\mathrm{E}_{\mathrm{kin}}=1456\right.$ $\mathrm{eV}$ ) from the top layer of $\mathrm{W}$ atoms of $\mathrm{O} / \mathrm{W}(110)(1 \times 1) \times 12$ : (a) experimental data; (b) multiple scattering theory with oxygen atoms occupying the threefold hollow sites at the optimized structure parameters of $\mathrm{z}=0.91 \AA$ and $\ell=1.57 \AA$. 

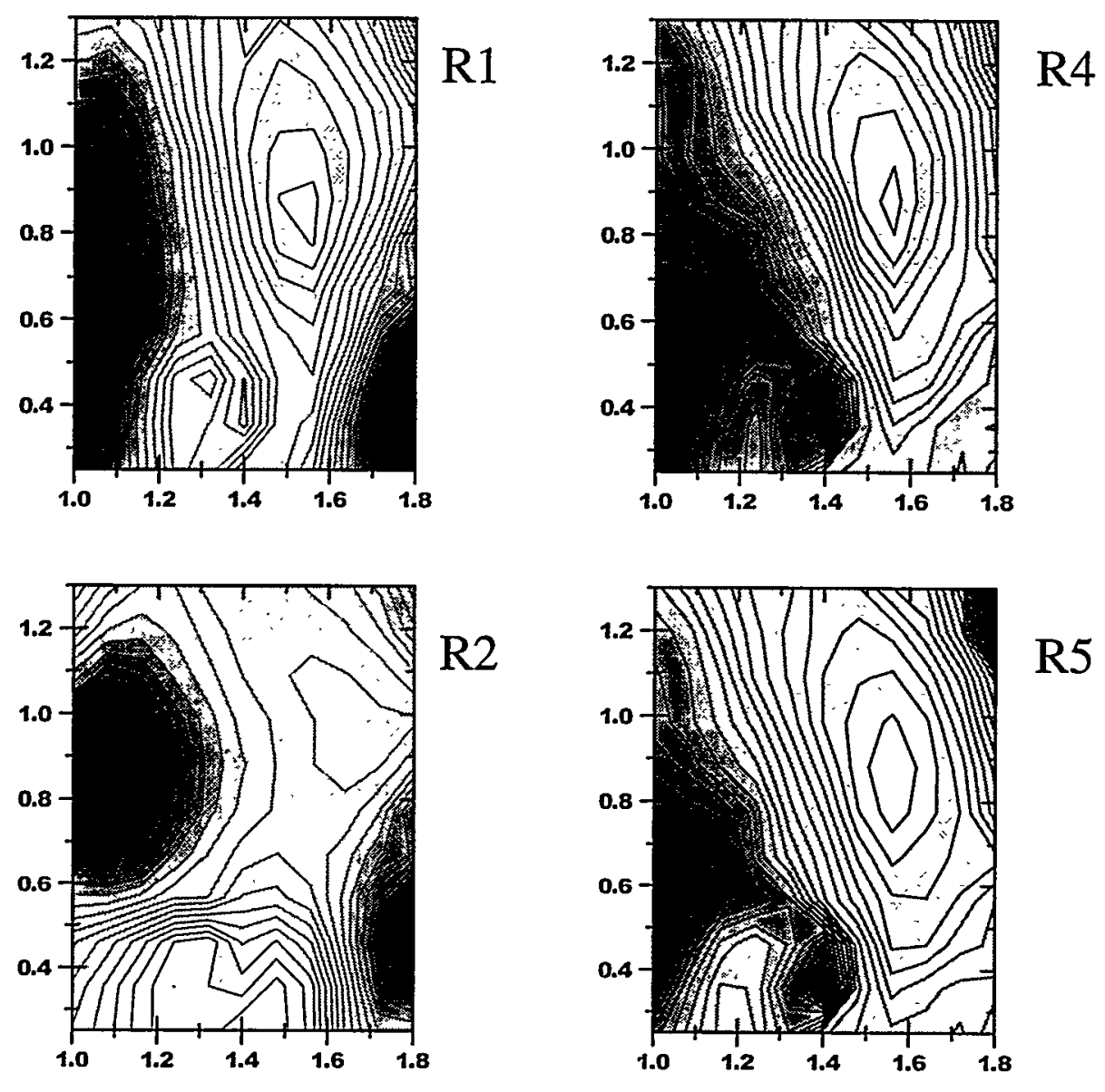

R5
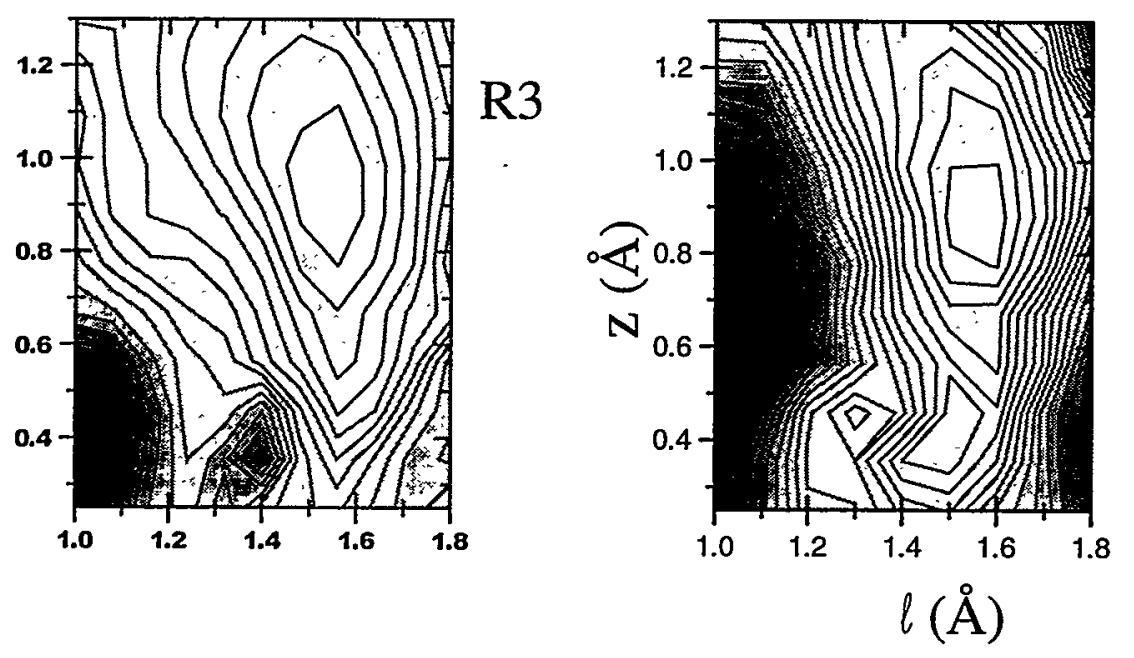

$\mathrm{R}_{\text {sum }}$

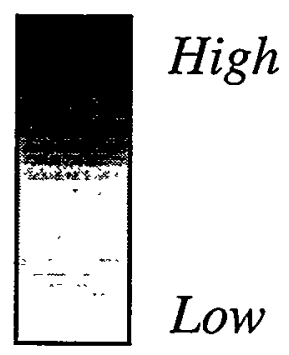

Figure 4.6. Two dimensional R-factor comparison between experiment and theory. Theoretical lateral position $\ell$ ranges from $1.0 \AA$ to $1.8 \AA$, with $1.67 \AA$ corresponding to equal bond distances between the oxygen atom and each tungsten atom in the pseudothreefold hollow site. Theoretical height of the oxygen layer $\mathrm{z}$ ranges from 0.3 to $1.3 \AA$. 


\author{
Chapter 5 \\ Kinetics and Atomic Structure of $O$ Adsorption on W(110) from \\ Time- and State- Resolved Photoelectron Spectroscopy \\ and Full-Solid-Angle Photoclectron Diffraction
}

\title{
5.I. INTRODUCTION
}

Oxygen adsorption on tungsten has a long history of investigation and was in fact one of the first surface adsorption systems to be studied [1-4]. Many experimental techniques have been applied to this system and an extensive review of many prior studies concerning it exists [5]. We will focus here on the close-packed W(110) surface, which has also been studied previously by many methods [5], including some investigations of particular relevance to our work using low energy electron diffraction (LEED) [6-8] scanning tunneling microscopy (STM) [9], and high-resolution core-level photoelectron spectroscopy [10-14]. These studies permit concluding that the oxygen adsorption site is in the pseudo-threefold hollow on W(110) [9], as shown for various possible adsorption geometries in Fig. 5.1. One LEED determination of the position of the oxygen atom in an ordered $\mathrm{p}(2 \times 1)$ structure (cf. Fig. 5.1(b)) has also been performed [7]. The ordering kinetics, phase diagrams, and site-to-site interaction parameters for forming overlayer structures with longer-range order or disorder have also been studied by finite size scaling theory [15] and by a spot profile analysis of superlattice LEED spots [8]. In spite of this large body of work, however, several important questions remain as to the basic kinetics of adsorption and the precise atomic structures involved. For 
example, under certain conditions (an example of which is described below) no longrange ordered structures are formed, and so there exist no LEED superstructure spots with which to study kinetics, even though final coverages can reach 0.5 monolayers (ML) or more. Such structures lacking long-range order are in fact expected to be the rule rather than the exception in surface reactions, and so it is of interest for future surface science research in general to develop experimental techniques to study them. We have thus applied chemical state- and time- resolved photoelectron spectroscopy (PS) and photoelectron diffraction (PD) to the $\mathrm{O} / \mathrm{W}(110)$ system as a first illustration of what these combined methods can provide in the way of additional information on surface reaction kinetics and atomic structure for situations lacking long-range order. Chemical state specificity is provided via the chemical shifts in high-resolution $\mathrm{W} 4 \mathrm{f}_{7 / 2}$ spectra, which have been studied for some time [10-12]. Time resolution is provided by the fact that such spectra can now be accumulated in as little as 10-30 seconds by taking advantage of third-generation high-brightness synchrotron radiation $[13,14,16]$. By studying the decay and growth with time of various chemically shifted peaks in the $\mathrm{W} 4 \mathrm{f}_{7 / 2}$ photoelectron spectra, as recently also discussed for oxygen on W(110) by Riffe and Wertheim [14], we are thus able to draw quantitative conclusions concerning the reaction kinetics involved. We have also modeled the time-resolved data in the simple limit that the oxygen atoms are not allowed to diffuse on the surface, using a simple Monte-Carlo simulation. Finally, we have also measured full-solid-angle photoelectron diffraction patterns for two oxygeninduced W states, and by comparing experiment with theoretical photoelectron diffraction calculations, are able to accurately determine the local atomic structures involved in this adsorption process for the first time. The relationship of these structures to prior work [7] 
and to a very recent PD study of the (1x1) oxygen structure formed at $1.0 \mathrm{ML}$ coverage [17] is also considered.

\section{II. EXPERIMENTAL METHODOLOGY}

Our experiments were performed on bend-magnet beamline 9.3.2 of the Advanced Light Source (ALS) at the Lawrence Berkeley National Laboratory [18(a)] using its advanced photoelectron spectrometer/diffractometer experimental endstation [18(b)]. This facility has both a high-resolution spherical grating monochromator and a highresolution/high-throughput Scienta SES 200 spectrometer. The W(110) sample was prepared by an initial oxygen treatment at a temperature of $1200^{\circ} \mathrm{C}$ and a pressure of $1 \times 10^{-7}$ Torr $\mathrm{O}_{2}$ to reduce the amount of carbon from the near-surface region. This was followed by repeated flashing at up to $2200^{\circ} \mathrm{C}$ by electron bombardment heating. A clean $\mathrm{W}(110)$ surface (as judged by conventional $\mathrm{x}$-ray PS analysis and a sharp (1x1) LEED pattern) was then reacted in a dynamic way with oxygen at a pressure of $3 \times 10^{-9}$ Torr and at three temperatures: $\mathrm{T}=298 \mathrm{~K}$ (room temperature), $360 \mathrm{~K}$, and $593 \mathrm{~K}$. The base pressure in the chamber before beginning to leak in oxygen was $1.3 \times 10^{-10} \mathrm{Torr}$, and, at this pressure, the influence of residual hydrogen on the resulting surface reaction kinetics is expected to be negligible [19]. For example, even if all of the residual gas were hydrogen (a conservative assumption), the reaction with oxygen at $3 \times 10^{-9}$ Torr would still be $30 / 1.3 \sqrt{2 / 32}=5.8$ times faster based on a simple surface collision rate argument. High resolution W $4 f_{7 / 2}$ spectra in which the total instrumental contribution to linewidth is estimated to be $80 \mathrm{meV}$ were then taken every 23 seconds over a total exposure time to 
the high pressure of oxygen of 70 minutes. This corresponds to a total of about 180 spectra obtained up to a total oxygen exposure of 12.6 Langmuirs $(L)$. Finally, after the 70-minute exposure to oxygen in the room temperature experiment, we performed fullsolid-angle state-resolved photoelectron diffraction measurements, as detailed below.

The experimental geometry is shown in Fig. 5.2. The angle $(\alpha)$ between the linearly-polarized incident radiation and the electron emission direction was kept fixed at $70^{\circ}$, with the polarization vector $\varepsilon$ lying in the plane of the [110] surface normal and the outgoing photoelectron wave vector $\mathbf{k}$. The sample was rotated on two perpendicular axes using a specially-constructed goniometer so as to vary the polar angle of emission $\theta$ (a takeoff angle measured relative to the surface plane) and the azimuthal angle of emission $\phi$ (measured relative to the W[[1 $\overline{1} 0]$ direction lying in the surface plane). The excitation energy used was $100 \mathrm{eV}$, via second order light with the monochromator set at $50 \mathrm{eV}$, resulting in $\mathrm{W} 4 \mathrm{f}_{7 / 2}$ photoelectrons with kinetic energies of approximately $69 \mathrm{eV}$. This photon energy was selected to maximize flux while maintaining high resolution and surface sensitivity (via the small electron inelastic attenuation length).

The time-resolved photoelectron spectra were taken at an angle of $45^{\circ}$ along the [001] azimuthal direction [ $\phi=90^{\circ}$ in our convention]. This corresponds to bulk photoelectron emission along [111] chains of tungsten atoms at an internal angle of $54.7^{\circ}$, after taking into account refraction of the photoelectrons as they exit the solid into the vacuum due to the inner potential $\mathrm{V}_{\mathrm{o}}$ of approximately $12.5 \mathrm{eV}$ [20]. The timeresolved intensities were then corrected for any variations in flux with time due to the 
decay of current in the storage ring, and normalized to remove slight effects on them due to photoelectron diffraction (by making use of the PD data obtained at the end of the exposure). The LEED patterns during and after all three of these time-dependent studies did not show any superstructure spots, but simply exhibited a progressive smearing of the clean W (1x1) pattern; the clean (1x1) and the three patterns after exposure are shown in Figs. 5.3 (a)-(d). Thus, no oxygen-induced long-range-ordered structures were formed on our surface under these reaction conditions.

The W $4 f_{7 / 2}$ photoelectron diffraction data were measured over nearly $80 \%$ of the $2 \pi$ solid angle above the surface, spanning the takeoff angle range from $\theta=9^{\circ}$ to $70^{\circ}$ (with $90^{\circ}=$ normal emission) in $3^{\circ}$ steps and the $180^{\circ}$ azimuthal angle range from $\mathrm{W}$ $[\overline{1} 10]$ to $[1 \overline{1} 0]$. The azimuthal step size was also adjusted with takeoff angle such that the final data set consisted of spectra representing roughly equal solid-angle increments. Angles below $9^{\circ}$ were not used due to the much lower count rates and thus longer counting times involved. Angles above $70^{\circ}$ were omitted due to experimental time limitations, but in any case this region of solid angle is also not as sensitive to structure as the other $80 \%$ over which we did measure. The analyzer acceptance angle was approximately a $\pm 5^{\circ}$ cone, with the spectrometer lens being operated in standard mode with no pre-collimators in place. Due to the symmetry of the crystal and the oxygen overlayers (which will be found to form in two mirror-related domains), only a $90^{\circ}$ azimuthal scan was needed to provide all the information over the full $360^{\circ}$ azimuthal range. However, in order to increase accuracy and average over any small sample misalignments, $180^{\circ}$ azimuthal scans were made and this data was then appropriately 
mirror-averaged into $90^{\circ}$ and then repeated four times to obtain the full $360^{\circ}$ azimuthal patterns that will be shown here. During the diffraction measurements, the beamline and spectrometer permitted taking a high-resolution spectrum in about 27 seconds, or just slightly longer than in the kinetics study. Fitting these spectra with appropriate inelastic backgrounds of Shirley type and peak shapes of Voigt type then permitted deriving separate diffraction patterns from each chemically-shifted peak. Any variations of radiation flux with time due to decay in the storage ring current were normalized out in determining the final diffraction intensities $I(\theta, \phi)$.

$\mathrm{O}$ on $\mathrm{W}(110)$ can form several different long-range ordered structures depending on the coverage, the exposure and annealing temperature, and the pressure at which the adsorption takes place $[8,9,17]$. As important background data for this study, we have thus also obtained high-resolution $\mathrm{W} 4 \mathrm{f}_{7 / 2}$ spectra for the three long-range-ordered $\mathrm{O}$ structures which form on $\mathrm{W}(110): \mathrm{p}(2 \times 1)$ and $\mathrm{p}(2 \times 2)$ [8], as well as the saturation coverage (1x1) with a (1x12) superlattice that requires high-temperature annealing for its formation $[9,17]$. These three structures formed easily on our clean W(110) surface under the following conditions: for the $\mathrm{p}(2 \times 1)$, we exposed the surface to $1.0 \times 10^{-7}$ Torr of oxygen for 30 seconds at room temperature, for the $\mathrm{p}(2 \times 2)$ we exposed the surface to $5.0 \times 10^{-7}$ Torr oxygen for 12 seconds at room temperature, and for the (1x1) we exposed the surface to $1.3 \times 10^{-6}$ Torr of oxygen and a temperature of $700^{\circ} \mathrm{C}$ for 10 minutes. Figs. 5.1 (b)-(d), show schematic drawings of these different structures, with the oxygen atoms in one of two possible pseudo-threefold sites related by a mirror reflection along [ 110$]$. The second mirror-related site thus leads to a second domain type for any of the three 
ordered structures. The corresponding experimental LEED patterns for the clean surface and the three ordered structures are shown in Figs. 5.3(d)-(g). The coverages for complete stable single-domain overlayers are as follows: $\mathrm{p}(2 \times 1)$ yields $1 / 2$ monolayer, $\mathrm{p}(2 \times 2)$ yields $3 / 4$ monolayer, and (1x1) yields a full monolayer coverage, with a monolayer corresponding to $1.42 \times 10^{15}$ atoms $/ \mathrm{cm}^{2}$, the density of tungsten atoms in the (110) plane. Depending on how domain boundaries are formed the actual coverages may be slightly higher or lower than these numbers. If these ordered structures are now viewed from the $\mathrm{W}$ atoms, we also note that there are four distinct types of nearestneighbor oxygen bonding environments, corresponding to $\mathrm{W}$ atoms bound to one $\mathrm{O}$ atom only (the distinct $\mathrm{O} 1 \mathrm{a}$ and $\mathrm{O} 1 \mathrm{~b}$ types), $\mathrm{W}$ atoms bound to two $\mathrm{O}$ atoms (O2), and $\mathrm{W}$ atoms bound to three $\mathrm{O}$ atoms (O3), as discussed previously in connection with highresolution core spectra [11,14]. Fig. 5.1(a) also shows a sparsely covered surface in which there is a mixture of both oxygen domain types (as short-range-ordered structures) and all four W atom types.

As one final aspect of our experimental data, we have also determined the variation of total oxygen coverage with time for our room temperature reaction conditions, by using a standard $\mathrm{Al} \mathrm{K} \alpha \mathrm{x}$-ray source and measuring the ratio $\mathrm{I}_{\mathrm{Ols}} / \mathrm{I}_{\mathrm{W} 4 \mathrm{f}}$. These measurements were carried out both on the same W(110) surface at the end of our 70 minute exposure only, and on a second W(110) surface over the full 0-70 minute time interval. In the first measurement, which was analyzed using standard equations and photoelectric cross sections for quantatitive XPS [21], we found a final O coverage of $0.57 \pm 0.10 \mathrm{ML}$ at the end of 70 minutes. Note that this is above the saturation coverage if 
the most stable $\mathrm{p}(2 \times 1)$ structure were to be formed. In measurements on a second W(110) surface which may have had a different degree and orientation of slight miscut, full-solid-angle $\mathrm{O}$ 1s and $\mathrm{W}$ 4f data was analyzed through angle-averaged ratios so as to minimize diffraction effects, and this showed a smooth uptake curve and a final coverage of $0.47 \pm 0.08 \mathrm{ML}$ that is somewhat lower than for the principal surface studied here, but within our accuracy estimate of being the same value.

\section{II. RESULTS AND DISCUSSION}

\section{High-resolution spectra:}

Fig. 5.4 now shows photoelectron spectra for the clean surface and the three ordered oxygen overlayer structures mentioned in the last section, as taken at a kinetic energy of $\sim 69 \mathrm{eV}$ and with the photoelectron emission below the surface along the [111] direction (as judged by allowing for surface refraction due to the inner potential). For the clean surface, there are two well-known peaks corresponding to the bulk W atoms and the $\mathrm{W}$ atoms at the clean surface, with a surface core level shift of $-320 \mathrm{meV}$ in binding energy and the surface peak thus at a higher kinetic energy with respect to the bulk peak [10-12]. Based on prior analyses $[11,13,14]$, the $\mathrm{p}(2 \times 1)$ spectrum is known to consist of two peaks: one mainly due to bulk tungsten atoms and a chemically shifted peak for which each tungsten atom is bound to two oxygen atoms (O2). In addition, other chemically-shifted peaks that are more difficult to resolve correspond to tungsten atoms bound to one oxygen atom $(\mathrm{O} 1=\mathrm{Ola}$ and $\mathrm{O} 1 \mathrm{~b})$ and these are found very close to the bulk peak. These peaks are thought to lie on either side of the bulk peak and they result from 
the two different local geometries associated with tungsten bound to one oxygen, as indicated by $\mathrm{O} 1 \mathrm{a}$ and $\mathrm{O} 1 \mathrm{~b}$ in Fig. 5.1. For the $\mathrm{p}(2 \times 1)$ structure shown in Fig. 5.1(b), the Ola exists only at the edges of the domain, while the O1b comprises exactly $50 \%$ of the top layer of $\mathrm{W}$ atoms inside the domain, with $\mathrm{O} 2$ comprising the other half. Thus, we can imagine that, for the large domains characteristic of a surface showing a sharp $\mathrm{p}(2 \times 1)$ LEED pattern, the peak O2 in Fig. 5.4(b) has a "twin" of nearly equal intensity that corresponds to the $\mathrm{O} 1 \mathrm{~b}$ intensity contribution to the "Bulk + O1" peak. In our study, the O1 peaks are not clearly resolved due to their smaller chemical shift from the bulk peak (approximately a $-160 \mathrm{meV}$ shift to lower kinetic energy for $\mathrm{Ola}$ and $\mathrm{a}+60$ to $+80 \mathrm{meV}$ chemical shift to higher kinetic energy for O1b) [10-12,14]; and, for the O1a peak, its relatively low intensity compared to the bulk peak. For these reasons, we have in the subsequent analysis of our data simply grouped these peaks with the bulk peak, although we present in Fig. 5.5 spectra in which all six components thought to be present (surface, bulk, O1a, O1b, O2, and O3) have been resolved by peak fitting. Returning to Fig. 5.4, we note that the $\mathrm{p}(2 \times 2)$ spectrum in (c) consists of three peaks: the bulk peak (again with unresolved $\mathrm{O} 1$ contributions), the chemically-shifted $\mathrm{O} 2$ peak, and a third chemicallyshifted peak for which each tungsten atom is bonded to three oxygen atoms (O3). The (1x1) spectrum in Fig. 5.4(d) by contrast consists of only two peaks: the bulk peak and the chemically shifted $\mathrm{O} 3$ peak.

The time-resolved $\mathrm{W} 4 \mathrm{f}_{7 / 2}$ spectra taken at room temperature during the reaction of oxygen with the surface are now also shown in Fig. 5.5. In Fig. 5.5(a) is a spectrum taken for a nearly clean $\mathrm{W}(110)$ surface just after the onset of adsorption, at only 30 
seconds of exposure thus corresponding to about $0.09 \mathrm{~L}$ oxygen exposure. This figure shows two well-resolved peaks, with a binding energy shift of about $-320 \mathrm{meV}$ between them and the peak at higher kinetic energy (lower binding energy) being attributed to the top layer of tungsten atoms with no overlying adsorbate [10-12]. (See also discussion of the clean surface structure as studied by full-solid-angle PD elsewhere [21]). The spectra have been analyzed by peak fitting so as to derive approximate estimates of the relative contributions of $\mathrm{Ola}$ and $\mathrm{O} 1 \mathrm{~b}$ to the "bulk +01 " peak, as well as more precise determinations of the surface, $\mathrm{O} 2$, and $\mathrm{O} 3$ peaks, and the results are shown as the several curves under the experimental spectrum. Figs. 5.5(b), (c), (d), and (e) show later spectra taken at 10 minutes, 20 minutes, 35 minutes, and 70 minutes, respectively, for our room temperature data. By 35 minutes or an exposure of $6.3 \mathrm{~L}$, the surface peak has disappeared, indicating that all atoms in the top layer of tungsten are bound to at least one atom of oxygen (or perhaps also hydrogen--but we expect this to be to a much lower degree, as noted previously $[12-14,19])$. There are two very evident chemically-shifted peaks in Fig. 5.5(d) at 35 minutes: one at a higher binding energy with $+350 \mathrm{meV}$ shift relative to bulk that we assign to a first and more weakly bound chemisorbed species (O2). The second weaker chemically shifted peak has a separation of $+625 \mathrm{meV}$ from the bulk. We assign this peak to a second and more strongly bound "incipient-oxide-like" species (O3). This $+625 \mathrm{meV}$ peak is found to increase in relative intensity in going up to 70 minutes exposure, as shown in Fig. 5.5(e), and by the end of the exposure it is more important than the $\mathrm{O} 2$ peak. This $\mathrm{O} 3$ chemical state is also dominant at $1.0 \mathrm{ML}$ oxygen coverage, as shown already in Fig. 5.4(d), but the longer-range order present in this structure has increased the chemical shift to $730 \mathrm{meV}$. (See also discussion of this $1 \mathrm{ML}$ 
structure elsewhere [21].) Figs. 5.5(b), (c), (d), and (e) also make it clear that the +350 $\mathrm{meV}$ state grows in first, but that the $+625 \mathrm{meV}$ state becomes dominant by the end of the time interval for room temperature reaction.

In order to relate our values for various surface and chemical shifts to prior work, we compare various values for these shifts in Table 1. There is in general excellent agreement, especially with the more recent work by Riffe and Wertheim [14]. Note also the change in these shifts with coverage, presumably due to effects beyond the nearestneighbor interactions that are used to establish the designations $\mathrm{O} 0=$ clean surface, $\mathrm{O}$, $\mathrm{O} 2$, and $\mathrm{O} 3$.

\section{Kinetics analysis:}

Fitting peak shapes to our set of time-resolved spectra now permits determining the quantitative time dependence of all four of the peak intensities observed (Surface, Bulk+O1, O2, and O3), as shown in Fig. 5.6. The intensities shown here have been corrected slightly so as to be more closely proportional to the number of $\mathrm{W}$ atoms of a given type by allowing for effects from photoelectron diffraction. These small corrections were determined from the diffraction data obtained at the end of the 70 minute exposure at room temperature (see discussion below), with the ratios of the Bulk+O1, $\mathrm{O} 2$ and $\mathrm{O} 3$ peaks thus being adjusted to account for diffraction. The intensities have also been corrected for the usual drop in flux due to storage ring beam decay with time. 
We first consider the room-temperature time-resolved results in Fig. 5.6(a). From this figure, we can see that the decay of the surface peak occurs over the first 10-15 min. (thus terminating at an oxygen exposure of 1.8-2.7 L) and that it is accompanied by a growth of the Bulk+O1 peak. This growth is due to contributions from the unresolved Ola and O1b peaks that are included in the Bulk+O1 peak (cf. Figs. 5.5(b),(c)). As the Bulk +01 peak begins to decrease, the $+350 \mathrm{meV}$-shifted $\mathrm{O} 2$ peak begins to grow, reaching a maximum at about $30 \mathrm{~min}$. Then, the $+625 \mathrm{meV} \mathrm{O} 3$ peak begins to grow in, at the expense of the $+350 \mathrm{meV} \mathrm{O} 2$ peak, becoming more important than it at about 60 minutes. These results thus show a clear linkage in the kinetics of the adsorption processes leading to these various bonding states of $\mathrm{W}$.

Fig. 5.6(b) and (d) show analogous state-resolved kinetics curves at the elevated temperatures of $\mathrm{T}=360^{\circ}$ and $\mathrm{T}=598^{\circ}$. With an increase in temperature, it is interesting that these reactions occur in a different manner (probably due to the increased oxygen mobility on the surface) and in general on a slower time scale for overall oxygen uptake (probably due to a greater rate of desorption). At both higher temperatures, the $\mathrm{O} 2$ spectral component representative of $\mathrm{W}$ bound to two $\mathrm{O}$ atoms grows in and levels off, implying that the final majority local bonding structure is of $\mathrm{p}(2 \mathrm{x} 1)$ type. The formation of $\mathrm{W}$ bound to three $\mathrm{O}$ atoms, as represented by the $\mathrm{O} 3$ component, is strongly suppressed at both higher temperatures: at $\mathrm{T}=360^{\circ}$, this component barely appears, while at $\mathrm{T}=598^{\circ}$ it has negligible intensity. These state-resolved measurements thus exhibit a striking change in the evolution of the local bonding with time as temperature is increased. The LEED patterns for these three different exposure temperatures by contrast show no 
significant differences after 70 minutes of oxygen exposure, as shown in Figs. 5.3(a)-(c). These data demonstrate the unique ability of state- and time-resolved PS to study kinetics for structures which do not exhibit long-range order and are thus difficult or impossible to clearly identify in LEED.

In order to have some more quantitative insight on the actual kinetics that are taking place, we have carried out Monte-Carlo simulations for the simple limiting case in which oxygen atoms (somehow formed by the dissociation of an $\mathrm{O}_{2}$ precursor on the surface) are not allowed to move once they have been adsorbed on the surface. This first assumption is reasonable, as prior work suggests that the diffusion coefficient of $O$ atoms on the surface is very low up to room temperature. In fact in some early studies it was believed that the $\mathrm{O}$ atoms were essentially immobile for temperatures below $600 \mathrm{~K}$, and this is essentially confirmed in more recent work $[4,5,22]$. For example, the lowest temperatures at which diffusion has been measured with reasonable accuracy are $417 \mathrm{~K}$ to $660 \mathrm{~K}[4,22]$. In this prior work, it was found that, for a coverage of about $0.18 \mathrm{ML}$, the diffusion constant pre-factor $D_{0}$ is $2.7 \times 10^{-5} \mathrm{~cm}^{2} \mathrm{~s}^{-1}$ and the activation energy for diffusion $E_{d}$ is $16.5 \mathrm{kcal} \mathrm{mol}^{-1}$ [22]. For a coverage of about $0.54 \mathrm{ML}$ near the maximum reached in our study, $D_{0}$ increases to a maximum of about $2.7 \times 10^{-5} \mathrm{~cm}^{2} \mathrm{~s}^{-1}$, but the activation energy also increases to about $25.2 \mathrm{kcal} \mathrm{mol}^{-1}$. In the low coverage regime and at room temperature, these numbers translate via the simple theory for tracer diffusion [4] into a hopping frequency between two adjacent pseudo-threefold hollow sites of $0.08 \mathrm{~s}^{-1}$ that is low enough to justify our assumption of immobile oxygen atoms at room temperature. We also in our model do not explicitly consider the initial process of 
dissociation of $\mathrm{O}_{2}$ that is thought to occur on W(110) via a mobile precursor state which has been assumed to preferentially bond to the oxygen-covered parts of the surface [5]. Rather we simply assume that each collision of $\mathrm{O}_{2}$ from the gas phase with the surface deposits two $\mathrm{O}$ atoms at random and uncorrelated positions on the surface; this is the Monte Carlo aspect of the model. The last is an extreme assumption, but we will see that the model nonetheless overall yields results that are in surprisingly good agreement with experiment. Also, a recent STM study of oxygen adsorption on the close-packed Al(111) surface by Brune et al. [23] indicates a similar very low mobility of oxygen atoms and a lateral separation of the atoms upon molecular dissociation that is consistent with one of the assumptions of our model. Furthermore, from the attractive and repulsive interactions known to exist in leading to the formation of the various ordered structures of oxygen on $\mathrm{W}(110)[5,8,15]$, we do not allow an oxygen atom to adhere to the surface unless it can occupy a pseudo-threefold-hollow site that is sufficiently far from any previously occupied site. That is, the oxygen atoms are only allowed to occupy sites which can finally form a local (1x1) structure in the three-fold hollow-sites around each tungsten atom (cf. Fig. 5.1(d)), with this prohibiting these atoms from becoming too tightly packed on the surface. The allowed occupations are thus illustrated in Fig. 5.1(a), and for more ordered structures in Figs. 5.1(b)-(d). Our assumption of non-mobility of adsorbed oxygen atoms is thus more reasonable at lower temperatures, and this model is therefore expected to best describe the room temperature data.

The simulation starts off with a $100 \times 100$ matrix of empty threefold sites for the oxygen to adhere to. The oxygen molecules in the gas phase are assumed to have an 
average velocity as dictated by the Boltzman distribution in determining the collision rate with the surface and, as noted above, are simply assumed to dissociate upon collision with the surface so as to form two oxygen atoms at uncorrelated positions. If a given atom produced in this way is within the two-dimensional triangular "cell" centered on an allowed threefold hollow adsorption site, it is assumed to stick. At room temperature and at a pressure of $3.0 \times 10^{-9}$ Torr $\mathrm{O}_{2}$, there are $1.7 \times 10^{12}$ collisions $\mathrm{cm}^{-2} \mathrm{~s}^{-1}$, which are assumed in the calculation to produce $3.4 \times 10^{12}$ atoms $\mathrm{cm}^{-2} \mathrm{~s}^{-1}$, and we make use of this rate to put a real time scale on the Monte Carlo process. Calculations and experiment can thus finally be compared on the same time scale by comparing theory with experiment at the same total collisional exposures to $\mathrm{O}_{2}$. The first set of simulations allowed for only one domain to form (i.e. only one type of threefold hollow to be occupied) while the second simulation allowed formation of both domains as simply dictated by the initial (random) formation point of a given oxygen atom.

In analyzing the final results of these calculations, tungsten atoms that do not have any oxygen nearest neighbors are considered surface atoms. Tungsten atoms that have one nearest neighbor oxygen atoms are marked as $\mathrm{O} 1$ species, thus including sites of O1a and $\mathrm{O} 1 \mathrm{~b}$ type. Tungsten atoms with two nearest-neighbor oxygen atoms are marked as $\mathrm{O} 2$ species and tungsten atoms with three oxygen nearest-neighbors as $\mathrm{O} 3$ species. As a graphic indication of the time dependence of the structures formed during the time sequence of the calculation, we show in Figs. 5.7(a)-(c) the actual types of $\mathrm{W}$ atoms present at $10 \mathrm{~min}, 20 \mathrm{~min}$, and $40 \mathrm{~min}$; here, the blank surface areas represent $\mathrm{W}$ atoms not bound to $\mathrm{O}$, and the $\mathrm{O} 1, \mathrm{O} 2$, and $\mathrm{O} 3$ species are indicated in various codes. In these 
figures, we do not distinguish the two mirror-related domain types, although both have been allowed to form. In a second set of calculations, the same model was used, but with only one domain type allowed; this is potentially a physically meaningful reference as

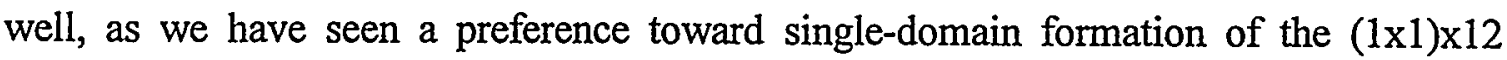
structure on this particular surface, as seen in the LEED pattern of Fig. 5.3(g), perhaps because of a slight miscut away from (110). The number of "bulk" tungsten atoms trivially remained constant, since the simulation only involved the top layer of tungsten atoms. In the experiment, we could not in practice accurately resolve the two $\mathrm{O} 1$ components from the bulk peaks and have thus grouped them into one intensity. In the simulation, we can of course resolve the two $\mathrm{O} 1$ states from the bulk and have included that data. As noted previously, these $\mathrm{O} 1$ states account for the sharp increase and then decrease in the Bulk+01 intensity seen in the first twenty minutes of the experimental data.

The final state-resolved results from the theoretical simulation are shown in Figs. 5.8(a) and (b) for single-domain and two-domain models, respectively. For the singledomain simulation shown in Fig. 5.8(a), we see that the different chemical states behave in a manner very similar to the experiment. The $\mathrm{O} 1$ peak is the first to dominate at the expense of the surface peak, with its maximum occurring at about 6 minutes. As time increases, the $\mathrm{O} 2$ peak comes to dominate as the primary species, with its maximum at 16 minutes and a crossover in importance with $\mathrm{O} 1$ at $10 \mathrm{~min}$. Finally, the $\mathrm{O} 3$ peak comes to be more important, with the crossover between $\mathrm{O} 2$ and $\mathrm{O} 3$ dominance occurring at about 20 min. For the two-domain simulation in Fig. 5.8(b) that we might expect to be a more 
accurate representation of reaction at a perfectly cut (110) surface, the curves have a qualitatively similar shape, but in general the maxima and crossover points occur at longer times. The $\mathrm{O} 1$ peak has a maximum at 8 minutes. The $\mathrm{O} 2$ peak reaches its maximum at about 49 minutes and then begins to fall slowly, and has a crossover with $\mathrm{O} 1$ at 20 minutes. The $\mathrm{O} 3$ peak steadily grows in importance until the end of the 70 minute time interval, and in additional calculations for much longer times not shown here is found to saturate at 3600 atoms, with the $\mathrm{O} 2$ peak leveling off at 4400 atoms; thus in the two-domain model, $\mathrm{O} 3$ never exceeds $\mathrm{O} 2$ in number and is finally about $82 \%$ of it. The behavior of these two sets of theoretical curves can now be compared to our roomtemperature experimental curves, for which $\mathrm{O} 1$ (as part of the Bulk+Ol peak) has a maximum at 9 minutes that is in excellent agreement with both sets of calculations; $\mathrm{O} 2$ has a maximum at 30 minutes that is intermediate between those of the single-domain and two-domain calculations, and a crossover with $\mathrm{O} 1$ at 20 minutes that is in excellent agreement with the two-domain calculations; and $\mathrm{O} 3$ shows the correct steadily increasing form in both calculations, and a crossover with $\mathrm{O} 2$ at 60 minutes that is intermediate between the two calculations. Given the extreme simplicity of our theoretical model, it nonetheless describes the observed state-specific kinetics remarkably well, with the possible preference for one of two domains on our particular surface helping to explain why some features of the single-domain theory (in particular, the crossover of $\mathrm{O} 2$ and $\mathrm{O} 3$ in importance at longer times) fit the experiment better. From this analysis, it is thus reasonable to suppose that the oxygen atoms in our particular lowpressure reaction condition are essentially immobile once they have found a threefold hollow site to sit in, in agreement with prior work $[5,22]$. We stress also that the gas 
pressure at which the reaction takes place is a crucial variable, as we are able by going to a much higher exposure pressure on the same single-crystal surface to form well-ordered $\mathrm{p}(2 \times 1)$ and $\mathrm{p}(2 \times 2)$ phases, as discussed previously. Thus, the much-discussed phase diagram for this surface $[5,8,15]$ really has one more variable in it: the gas pressure during exposure, and it would be interesting in future studies to explore this in more detail.

The total oxygen coverage versus time is also directly derivable from these model calculations, and these results are shown as solid curves in Figs. 5.8(a),(b). With a single domain, the total coverage approaches $1.0 \mathrm{ML}$ at $70^{\circ}$ minutes, which is reasonable in view of the dominance of $\mathrm{O} 3$ type $\mathrm{W}$ atoms. The two-domain calculations by contrast yield about $0.74 \mathrm{ML}$ at 70 minutes, which is in fact closer to the measured final coverage estimate for this surface of $0.57 \mathrm{ML}$. Thus, from all aspects of comparing experiment to these Monte Carlo calculations, it appears that the two-domain model provides a better description, but that a preference for one of the two domain types is also suggested and that this leads to certain features being better described better by the single-domain model.

In the other extreme limit of higher temperature (or by implication from our observations here also of higher pressure) for which the oxygen atoms are in some sense freer to move in order to minimize their energy, the most stable $\mathrm{p}(2 \mathrm{x} 1)$ overlayer should form at 0.5 monolayer coverage. Evidence for this is in fact found in our experimental data for higher temperatures, where only the $\mathrm{O} 1$, and then the $\mathrm{O} 2$ peaks grow in, while 
the $\mathrm{O} 3$ growth was minimal. However, we again note that no domains of $\mathrm{p}(2 \times 1)$ of sufficient size to be seen in LEED were found even in these cases (cf. Figs. 5.3(b),(c)), so that state-resolved PS provides a unique way of studying these states as small domains or even single sites of a given type. An additional interesting point concerning our two higher temperature data sets is that, for the lower adsorption pressures in the $10^{-9}$ Torr range at which these experiments were conducted, the lower rate of initial precursor adsorption and/or higher rate of atomic desorption leads to a lower uptake rate of oxygen on the surface and effectively causes the formation of the $\mathrm{O} 2$ and $\mathrm{O} 3$ states to occur much more slowly, even though the $\mathrm{O} 1$ formation, as judged by the Bulk+O1 peak, is very similar at all three temperatures.

As a final aspect of our kinetics analysis, we now consider the actual uptake of oxygen on the surface more quantitatively, including determinations of coverage and sticking coefficients versus time from our state-resolved data. In order to determine the oxygen coverage, $\Theta_{\mathrm{O}}$ in $\mathrm{ML}$, from our intensity measurements of the various $\mathrm{W}$ states, we have started with a formula derived recently by Riffe and Wertheim [14]:

$$
\Theta_{O}=\frac{\sum_{n=0,3} \frac{n}{3} I_{O n}}{\sum_{n=0,3} I_{O n}}
$$

where the summation is over the three resolved and, in our case one unresolved, spectral components, with $\mathrm{n}$ equal to the number of oxygen atoms that a given type of tungsten 
atom is bonded to ( 0 for surface, 1 for 01,2 for $\mathrm{O} 2$, and 3 for $\mathrm{O} 3$ ), $\mathrm{I}_{\mathrm{O}}$ is the intensity of that spectral component (ie. Surface $\equiv 00,01,02$, and 03 ), and the division by 3 is because each $\mathrm{O}$ atom is three-fold coordinate (since the oxygen atoms always sit in a three-fold hollow site). Since we were not able to accurately measure the O1 peaks, we determined $\mathrm{I}_{\mathrm{O} 1}$ by first assuming that the bulk intensity should remain essentially constant during the entire time interval of reaction. This assumption requires that the degree of inelastic and elastic scattering in the overlying layer of $\mathrm{W}$ +adsorbed $\mathrm{O}$ is very nearly constant over the full exposure range, which is quite reasonable in view of the expected dominance of the $\mathrm{W}$ atoms in this overlayer in producing both inelastic and elastic scattering of the photoelectrons emitted from the bulk. We have also checked this assumption, including the effects of diffraction, by measuring photoelectron diffraction patterns at the end of the room temperature experiment, and then multiplying the Bulk+O1, $\mathrm{O} 2$, and $\mathrm{O} 3$ peaks by appropriate values to compensate for the estimated effects of diffraction. Second, from the empirically more accurate two-domain kinetics calculation, we see that the ratio $01 / \mathrm{O} 2$ at the end of the $70 \mathrm{~min}$ is $\sim 0.4$. Thus, to estimate the contribution of $\mathrm{O} 1$ to $\mathrm{I}_{\mathrm{O} 1+\mathrm{Bulk}}$, we have simply gone to the end of the 70 minutes in the experiment, set $\mathrm{I}_{\text {expt@70 }}=\mathrm{I}_{\mathrm{O} 1+\mathrm{Bulk}}$ and then calculated $\mathrm{I}_{\mathrm{Bulk}}$ from (0.4) $\mathrm{I}_{\text {expt@70 }}$ in order to determine the assumed-constant bulk value over all times. Then for each data point as a function of time, we simply subtracted off the constant bulk value from Bulk+O1 to estimate the $\mathrm{O} 1$ intensity; these estimates are shown as the dashed curves in Fig. 5.6. Using these values for $\mathrm{I}_{\mathrm{O} 1}$ and the directly determined experimental values for $\mathrm{I}_{\mathrm{O} 0}, \mathrm{I}_{\mathrm{O} 2}$, and $\mathrm{I}_{\mathrm{O} 3}$ in Eq. 5.1, we finally obtain estimated coverages versus time as the curves shown in Fig. 5.9. The points are from the experimental data for the three 
temperatures, and the curves overlying them are 15-point smoothed values based on this data. The dot-dash curve is directly from the two-domain Monte Carlo simulation. Considering experiment first, the coverage initially increases rapidly as the sample first gets exposed to oxygen. As time increases and the number of possible sites decreases, the rate of change of coverage reduces, and all three experimental curves show a knee with fairly abrupt change of slope, occurring at about 16 minutes for $298 \mathrm{~K}$, at about 10-12 minutes for $360 \mathrm{~K}$, and at about 8 minutes for $593 \mathrm{~K}$. As the temperature increases, the amount of coverage at a given temperature also decreases, with only about $80 \%$ as much oxygen on the surface at $\mathrm{T}=360 \mathrm{~K}$ and $593 \mathrm{~K}$ as at $298 \mathrm{~K}$. It is also encouraging that the final coverage for $298 \mathrm{~K}$ at 70 minutes as calculated using Eq. 5.1 is in excellent agreement with that derived directly from the Monte Carlo simulation. The overall forms of the uptake curves are also very similar between experiment at $298 \mathrm{~K}$ and theory, although experiment shows more of a break in slope at approximately 20 minutes and a higher more nearly constant slope for longer times. In any case, the analysis in terms of Eq. 5.1 seems to provide a very good description of the lower-temperature data. All of the experimental curves are above theory for the lowest times measured, but this is probably due to the fact that it requires some time to raise the pressure from its base value to $3.0 \times 10^{-9}$ Torr of oxygen; thus some adsorption has already taken place before the "clock"for our state-resolved spectra has been started.

From Fig. 5.6(a) we note that the maximum in the $\mathrm{O} 2$ component for $298 \mathrm{~K}$ occurs at $\sim 30$ minutes. The corresponding experimental coverage from Fig. 5.9 now shows that this point arises for a coverage very close to $1 / 2$ monolayer. From this time 
onward, it is thus reasonable that any extra oxygen atoms adsorbed to the surface will convert $\mathrm{O} 2$ and $\mathrm{O} 1$ peaks into $\mathrm{O} 3$ peaks. This agrees with Fig. 5.6(a), which shows $\mathrm{O} 3$ beginning to grow in at the expense of the $\mathrm{O} 2$ and $\mathrm{O} 1$ peaks more strongly after about 30 minutes. At the two higher temperatures, we can see that the final coverages end at only slightly higher than $1 / 2 \mathrm{ML}$, thus helping explain why the $\mathrm{O} 3$ component is so weak in these cases.

By taking appropriate time derivatives of the results of Fig. 5.9, we can also easily determine the oxygen sticking coefficient, or probability that a colliding molecule will stay on the surface. The sticking coefficient was determined straightforwardly from our data by taking the derivative of coverage versus time curve, and then multiplying by the number of minutes needed to reach a coverage of 1.0 ML of oxygen atoms with unit sticking coefficient (i.e., $1.41 \times 10^{15}$ atoms $/ \mathrm{cm}^{2}$ ). The variation of both the experimental and theoretical sticking coefficients with coverage are shown in Fig. 5.10, where they are also compared to an average over several prior determinations of this type (Squares) [14]. We can see from this figure that the two higher-temperature sticking coefficients agree very well with prior work, showing a steep falloff from low to high coverage, and a very small value of about $5 \%$ by the time the saturation value of $0.5 \mathrm{ML}$ for the most stable $\mathrm{p}(2 \times 1)$ structure is reached. The sticking coefficient for $298 \mathrm{~K}$ by contrast stays higher at about $10 \%$ up to a coverage of $\sim 0.75 \mathrm{ML}$, and in general shows the same form as the simulation, lending further credence to this different behavior. This difference from prior room temperature data we attribute to the lower pressure at which the adsorption was carried out, as well as a possible difference in step and defect density from one surface to 
another. But the fact that we are able to effectively saturate this surface at $0.5 \mathrm{ML}$ by adsorbing at higher temperatures for which the oxygen atoms have higher mobility to form the $\mathrm{p}(2 \times 1)$ structure, and to form highly ordered structures for $\mathrm{p}(2 \times 1), \mathrm{p}(2 \times 2)$ and (1x1)x12 strongly suggests that we were studying a (110) surface with low step and defect densities.

\section{Surface structure determinations:}

As a final aspect of this study, we consider the local atomic structure around the two easily resolved types of $\mathrm{W}$ atoms: $\mathrm{O} 2$ and $\mathrm{O} 3$. To determine this, we have measured full-solid-angle photoelectron diffraction patterns for both the chemisorbed $\mathrm{O} 2$ and incipient-oxide $\mathrm{O} 3$ peaks in the $\mathrm{W} 4 \mathrm{f}_{7 / 2}$ spectrum at the end of the 70 min. adsorption, with these then permitting a direct and independent structural determination for the two W states involved. The experimental results for the $\mathrm{O} 2$ and $\mathrm{O} 3$ atoms are shown in Figs. 5.11(a) and 5.11(b), respectively.

Considering first the prior structural analyses that might be relevant to this system, we note that LEED has been used to determine the atomic structure of the $1 / 2 \mathrm{ML} p(2 \times 1)$ overlayer [7] that should consist entirely of the $\mathrm{O} 2$ state with a $350 \mathrm{meV}$ shift relative to the bulk (cf. Fig. 5.4(b)), but there is no other structure determination for $\mathrm{O} / \mathrm{W}(110)$ and LEED could not in any case be easily applied to the overlayer formed here, which lacks long-range order. The LEED values for the two structural parameters defined in Fig. 5.1(e) are $z=1.25 \AA$ and $\ell=1.67 \AA$, with the latter corresponding to equal bond distances from oxygen to all three $\mathrm{W}$ atoms in the pseudo-threefold hollow and not being 
varied in the R-factor fit to experiment. The only other related structure determination is for the $(1 \mathrm{x} 1)-\mathrm{x} 12 \mathrm{O}$ overlayer (cf. Fig. $5.3(\mathrm{~g}))$ in which the $\mathrm{O} 3$ state is dominant, which has been studied by both high-energy $\mathrm{x}$-ray $\mathrm{PD}$ and low-energy synchrotron radiation $\mathrm{PD}$, and yields values of $z=0.91 \AA$ and $\ell=1.57 \AA$, with the latter now corresponding to a lateral shift of the oxygen by $0.1 \AA$ away from the equal-bond-distance position along the [1110] direction (with the opposite shift in the second domain type). The present study thus represents the first attempt to determine the adsorption site when both the $\mathrm{O} 2$ and $\mathrm{O} 3$ structures occur simultaneously and with no long-range order.

The photoelectron diffraction data in Fig. 5.11(a) and (b) have been analyzed by comparing experiment to multiple scattering theory via five R-factors previously defined by Saiki et al. for use in PD studies [24(a)] together with a normalized sum of these five as an overall indicator of goodness of fit [24(b)]. The experimental data were also first converted to normalized chi functions, and that is what is presented in the figures. (See discussion elsewhere of these normalization and data analysis procedures [21].) The calculations were carried out using a newly completed multiple scattering cluster program [25] based upon the Rehr-Albers separable Green's function approach [26]. Beyond scattering phase shifts calculated in a standard way for muffin-tin potentials, the various non-structural inputs for these calculations were: electron inelastic attenuation length $=$ $\Lambda_{\mathrm{e}}=4.0 \AA$, inner potential $=\mathrm{V}_{\mathrm{o}}=11.0 \mathrm{eV}$ (reduced from the clean surface value of 12.5 $\mathrm{eV}$ to allow for the effect of adsorption), and Debye-Waller effects from an uncorrelated vibration calculation based on a Debye temperature of $400 \mathrm{~K}$. The atomic cluster used for the $\mathrm{O} 3$ calculation contained 100 atoms, including a full layer of $30(1 \times 1) \mathrm{O}$ atoms and 4 
layers of $\mathrm{W}$ underneath it. In the atomic cluster used for the $\mathrm{O} 2$ calculation, every other row of $\mathrm{O}$ atoms was removed, as shown in Fig. 5.1(c). Angular averaging was done in a two-step fashion by first summing theoretical intensities at 5 points equally distributed over a cone of $\pm 3^{\circ}$ (with one point weighted double in the center) and then applying a finer scale two-dimensional Gaussian smoothing with a full width at half maximum of $3^{\circ}$. This procedure was found to yield lower R-factors and better visual agreement between theory and experiment. Finally, in view of the preponderance of prior evidence that $O$ sits in the pseudo-threefold hollow site, we have only considered this in our structure optimization, although we have also allowed the lateral position as determined by $\ell$ to be varied. No relaxations or rumplings of the first-layer $\mathrm{W}$ atoms were considered, nor was the spacing between the first and second W layers varied. Thus, there may be other structural details that we have not considered, but we have in any case gone beyond a prior LEED investigation of the system [7].

The final optimized theoretical chi functions for the $\mathrm{O} 2$ and $\mathrm{O} 3$ data are shown in Figs. 5.11(c) and 5.11(d), respectively, and they are in excellent agreement with experiment. The variation of the summed, normalized overall $\mathrm{R}$-factor is also shown in Figs. 5.11(e) and 5.11(f) as a function of $\mathrm{z}$ and $\ell$, to provide some idea of the sharpness of the minimum. We finally find that the oxygen in $\mathrm{O} 2$ sites has an interlayer separation from the first $W$ layer of $z=0.97 \pm 0.05 \AA$ and a lateral position of $\ell=1.51 \AA \pm 0.05 \AA$, and that the oxygen in $\mathrm{O} 3$ sites has $\mathrm{z}=0.88 \AA \pm 0.05 \AA$ and $\ell=1.56 \AA \pm 0.05 \AA$. These results are shown in Table 5.2, in which they are compared to other previous structure determinations for similar structures. Our results show that the adsorption geometries for 
both $\mathrm{O} 2$ and $\mathrm{O} 3$ are very close to one another, with essentially identical lateral positions, but an indication of a smaller vertical distance on going from $\mathrm{W}$ bound to two oxygens to W bound to three oxygens. There is also excellent agreement between the present result for the 03 state and a recent determination for the $(1 \times 1) \times 12$ structure from low-energy scanned-angle $\mathrm{PD}$ data [21]. The resulting $\mathrm{W}-\mathrm{O}$ bond distances for our structures are: O2--two at $1.99 \AA$ and one at $1.79 \AA$, and O3--two at $1.93 \AA$ and one at $1.79 \AA$. Comparing these to W-O distances in bulk oxides of 1.46-2.94 [27] shows that they are reasonable.

\section{IV. CONCLUSIONS}

We have thus applied chemical state- and time- resolved photoelectron spectroscopy and photoelectron diffraction together for the first time in a study of oxygen adsorption on a metal surface. For the system $\mathrm{O} / \mathrm{W}(110)$, several conclusions can be drawn. For our surface and at an oxygen pressure of $3 \times 10^{-9}$ Torr, no adsorbate structures with long-range order are visible in LEED, but high-resolution W $4 f_{7 / 2}$ spectra nonetheless permit detecting "O2" $\mathrm{W}$ atoms bound to 2 oxygen atoms $(\mathrm{p}(2 \times 1)$-like in local structure), "O3" W atoms bound to three oxygen atoms ((1x1)- or incipient-oxidelike in local structure), as well as less well resolved "O1" W atoms bound to 1 oxygen atom. Highly ordered $\mathrm{p}(2 \times 1), \mathrm{p}(2 \times 2)$ and $(1 \times 1) \times 12$ structures could also be formed on this surface at higher pressures of exposure and/or with annealing at higher temperatures, indicating that the gas pressure is an important variable in determining the kind of adsorption structure formed. The individual time dependencies of the clean surface, 
unresolved Bulk+01, $\mathrm{O} 2$, and $\mathrm{O} 3 \mathrm{~W}$ intensities were studied by taking high resolution photoelectron spectra approximately every $20 \mathrm{~s}$ over a period of 70 minutes. Three temperatures of $298 \mathrm{~K}, 360 \mathrm{~K}$, and $593 \mathrm{~K}$ were studied. We find that the formation of the O3 state comes to be dominant at longer times for room temperature reaction, but its formation is strongly suppressed at the higher temperatures. A simple Monte Carlo model assuming that the adsorbed oxygen atoms have no mobility on the surface once they can occupy a given allowed pseudo-threefold hollow site is furthermore found to very well predict the qualitative form, and even some quantitative aspects, of the observed time-dependent intensities at $298 \mathrm{~K}$. The state-resolved intensities can furthermore be used to determine the total oxygen coverage versus time and hence the sticking probability versus coverage, with these results also agreeing with the Monte Carlo results in general, as well as with prior experimental determinations of sticking coefficients for this system. Finally, the local atomic geometries of oxygen in the shortrange-order $\mathrm{O} 2$ and $\mathrm{O} 3$ sites have been determined by means of full-solid-angle photoelectron diffraction measurements, combined with multiple scattering theory and Rfactor comparisons of experiment and theory. The positions thus determined are very similar to one another, and to the position of oxygen in the saturated (1x1)x12 structure as determined recently by high-energy and low-energy PD [17,21], with an indication that oxygen sits closer to the surface by $0.06-0.09 \AA$ when the $\mathrm{W}$ atoms are bound to three oxygens and that, in all structures studied, the oxygen is offset laterally in the pseudothreefold hollow site by $0.09-0.16 \AA$. 
This study thus demonstrates the considerable potential of chemical state- and time- resolved photoelectron spectroscopy at a third-generation synchrotron radiation source for studying surface reaction kinetics, as illustrated here by the multiple-state system involved in the initial stages of oxidation of clean W(110) and also by several recent studies by another group working at the Elettra facility in Italy [16]. Being able to simultaneously use photoelectron diffraction to determine the local atomic geometries around species with different chemical states, even in the absence of long-range order, as illustrated here for two different chemical states of $\mathrm{W}$, represents another unique capability that should be broadly applicable to various surface reactions and epitaxial growth processes. Finally, we have pointed out the importance of adsorption pressure in determining the kinds of structures which form on a given surface, and it is expected that this will have implications for many surface reactions and their kinetics. 


\section{References:}

[1] I. Langmuir, J. Am. Chem. Soc. 35, 105 (1913).

[2] L.H. Germer and J.W. May, Surf. Sci. 4, 452 (1966).

[3] E. Bauer, Surf. Sci. 7, 351 (1967).

[4] R. Gomer, Repts. Prog. Phys. 53, 917 (1990).

[5] C.R. Brundle and J.Q. Broughton, "The Initial Interaction of Oxygen with Welldefined Transition Metal Surfaces," appearing in The Chemical Physics of Solid Surfaces and Heterogeneous Catalysis, Vol. 3, p.131, D.A. King and D.P. Woodruff (Eds.), (Elsevier, 1990). The O/W(110) system is discussed on pp. $161-191$.

[6] E. Bauer and T. Engel, Surf. Sci. 71, 695 (1978).

[7] M.A. Van Hove and S.Y. Tong, Phys. Rev. Lett. 35, 1092 (1975).

[8] P.K. Wu, M.C. Tringides, and M.G. Lagally, Phys. Rev. B 39, 759 (1989).

[9] K.E. Johnson, R.J. Wilson, and S. Chiang, Phys. Rev. Lett. 71, 1055 (1993).

[10] G. Tréglia, M.C. Desjonguéres, D. Spanjaard, Y. Lassailly, C. Guillot, Y. Jugnet, Tran Minh Duc, and J. Leconte, J. Phys. C: Solid State Phys. 14, 3463 (1981). 
[11] D. Spanjaard, C. Guillot, M.C. Desjonguéres, G. Tréglia,J. Leconte, Surface Science Reports 5, 1 (1985).

[12] (a) Tran Minh Duc, C.Guillot, Y.Lassailly, J.Lecante, Y.Jugnet, and J.C. Vedrine, Phys. Rev. Lett. 41, 1425 (1978); (b) D.M. Riffe, G.K. Wertheim, and P.H. Citrin, Phys. Rev. Lett. 63, 1976 (1989).

[13] Abstract by R.X. Ynzunza et al. in the Advanced Light Source Compendium of Users Abstracts and Technical Reports, (1993-1996), Report LBNL-39981, UC411, pp. 398-401.

[14] D.M. Riffe, and G.K. Wertheim, Surf. Sci. 399, 248 (1998).

[15] B. Dunweg, A. Milchev, P.A. Rikvold, J. Chem. Phys. 94, 3958(1991).

[16] A. Baraldi et al., Surf. Sci. Lett. 385, 376 (1997) and refs. therein.

[17] H. Daimon, R.X. Ynzunza, J. Palomares, H. Takagi, and C.S. Fadley, Surf. Sci. 408, 260 (1998). McKinney, H. A. Padmore, C. S. Fadley, and D. A. Shirley, J. Elec. Spec. and Rel. Phen. 80, 401 (1996) and abstract by E.J. Moler et al. in the Advanced Light Source Compendium of Users Abstracts and Technical Reports, (1993-1996), Report LBNL-39981, UC-411, pp. 336-339; (b) C.S. Fadley, M.A. Van Hove, Z. Hussain, and A.P. Kaduwela, J. Elec. Spec. and Rel. Phen. 75, 273 (1995); R.X. Ynzunza et al., in the Advanced Light Source Compendium of Users Abstracts 
and Technical Reports, (1993-1996), Report LBNL-39981, UC-411, pp. 332-335; and C.S. Fadley et al., Prog. in Surf. Sci., 54, 341 (1997).

[19] D.M. Riffe, G.K. Wertheim, and P.H. Citrin, Phys. Rev. Lett. 65, 219 (1990).

[20] C.S. Fadley, in Synchrotron Radiation Research: Advances in Surface and Interface Science, Volume 1: Techniques, p. 421, R.Z. Bachrach (Eds.), (Plenum Press, 1992).

[21] R.X. Ynzunza, Ph.D. thesis (University of California-Davis, 1998) Chapters 3 and 4.

[22] (a) J.R. Chen and R. Gomer, Surf. Sci. 105, 48 (1981); (b) C. Uebing and R. Gomer, Surf. Sci. 381, 33 (1997).

[23] H. Brune, J. Wintterlin, R.J. Behm, G. Ertl, Phys. Rev. Lett., 68, 624 (1992).

[24] (a) R.S. Saiki, A.P. Kaduwela, M. Sagurton, J. Osterwalder, D.J. Friedman, C.S. Fadley, and C.R. Brundle, Surf.Sci. 282, 33 (1993); (b) R.S. Saiki, Ph.D. thesis (University of Hawaii, 1991).

[25] (a) Y. Chen, H. Wu, Z. Hussain, and D.A. Shirley, unpublished results; (b) Y. Chen, F.J. García de Abajo, A. Chassé, R.X. Ynzunza, A.P. Kaduwela, M.A. Van Hove, and C.S. Fadley, Phys. Rev. B, to appear; further details concerning this multiple scattering program are available at http://electron.lbl.gov/mscdpack/mscdpack.html. 
[26] J.J. Rehr and R.C. Albers, Phys. Rev. B 41, 81139 (1990).

[27] R.W.G. Wyckoff, in Crystal Structures, (Interscience Publishers, New York, (1963); Crystal Data Determinative Tables, Volume 4: Inorganic Compounds, Eds. H.M. Ondik and A.D. Mighell, (Published jointly by the U.S. Department of Commerce, National Bureau of Standards, and the JCPDS- International Centre for Diffraction Data, 1969). 


\section{Tables:}

TABLE 5.1--Comparison of W 4 f chemical and surface shifts for oxygen on W(110) as determined in various studies. All numbers are relative to the bulk binding energy, with representing a decrease in binding energy and + an increase. Units are meV.

\begin{tabular}{|c|c|c|c|c|c|c|c|c|}
\hline $\begin{array}{l}\text { O Surface } \\
\text { Structure: } \\
\text { Coverage } \\
\frac{\text { W Atom }}{\text { Type }}\end{array}$ & $\underset{W^{\#}}{\text { Clean }}$ & $\begin{array}{c}(1 \times 1) \times 12 \\
\mathrm{O}^{\#}: \\
1 \mathrm{ML}\end{array}$ & $\begin{array}{c}\mathrm{p}(2 \times 1)^{\#}: \\
1 / 2 \mathrm{ML}\end{array}$ & $\begin{array}{c}\mathrm{p}(2 \times 2)^{\#}: \\
3 / 4 \mathrm{ML}\end{array}$ & $\begin{array}{c}\text { Low } \\
\text { Coverage } \\
\mathrm{O}^{\#}: \\
0.2 \mathrm{ML} .\end{array}$ & $\begin{array}{c}\text { Low } \\
\text { Coverage } \\
\text { O: } \\
<0.1 \mathrm{ML} \\
{[12,14]}\end{array}$ & $\begin{array}{c}1 / 2 \mathrm{ML} \\
{[14]}\end{array}$ & $\begin{array}{c}\sim 1 / 2 \mathrm{ML} \\
{[11]}\end{array}$ \\
\hline Surface & -320 & ------ & ------ & ----- & -302 & -321 & $\ldots$ & ------ \\
\hline Ola & - & ------ & ----_- & -...- & - & -160 & -160 & --- \\
\hline Olb & - & ---- & -ב-- & - & - & +75 & +59 & +100 \\
\hline $\mathrm{O} 2$ & --- & --..- & +345 & +365 & +260 & +275 & +342 & +340 \\
\hline $\mathrm{O} 3$ & --. & +730 & +630 & +730 & -..- & -...- & +662 & +550 \\
\hline
\end{tabular}

\#From this study, with additional details in reference 21. 
TABLE 5.2--Comparison of various results for the atomic geometry of oxygen in the pseudo-threefold hollow on W(110)

\begin{tabular}{|c|c|c|c|}
\hline Structure & Method & $\mathrm{z}(\AA)$ & $\ell(\AA)$ \\
\hline $\mathrm{p}(2 \times 1)$ & LEED [7] & 1.25 & 1.67 \\
\hline$(1 \times 1) \times 12$ & $\mathrm{XPD}[17]$ & 0.84 & 1.52 \\
\hline$(1 \times 1) \times 12$ & low-energy PD [21] & 0.91 & 1.57 \\
\hline $\mathrm{O} 2$ & $\mathrm{PD}$ & 0.97 & 1.51 \\
\hline $\mathrm{O} 3$ & $\mathrm{PD} \#$ & 0.88 & 1.56 \\
\hline
\end{tabular}

\#From this study, with additional details in ref. 21. 

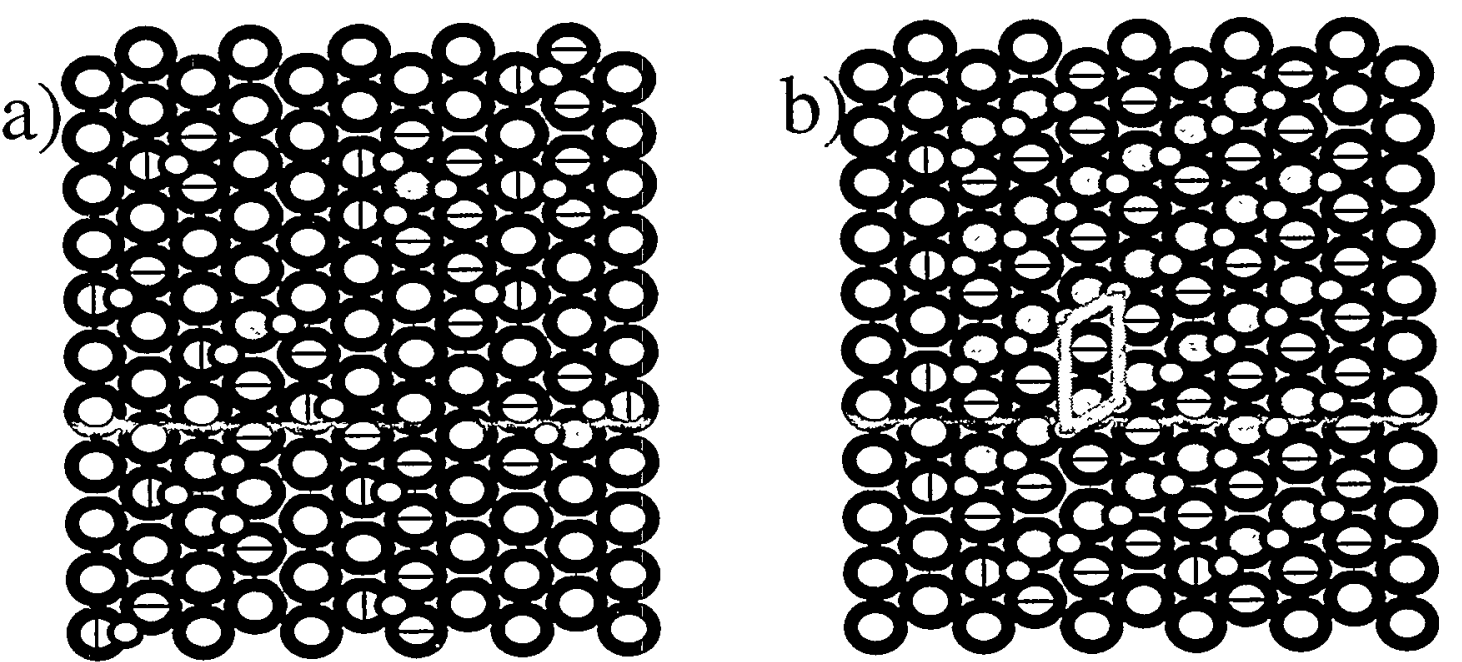

c)
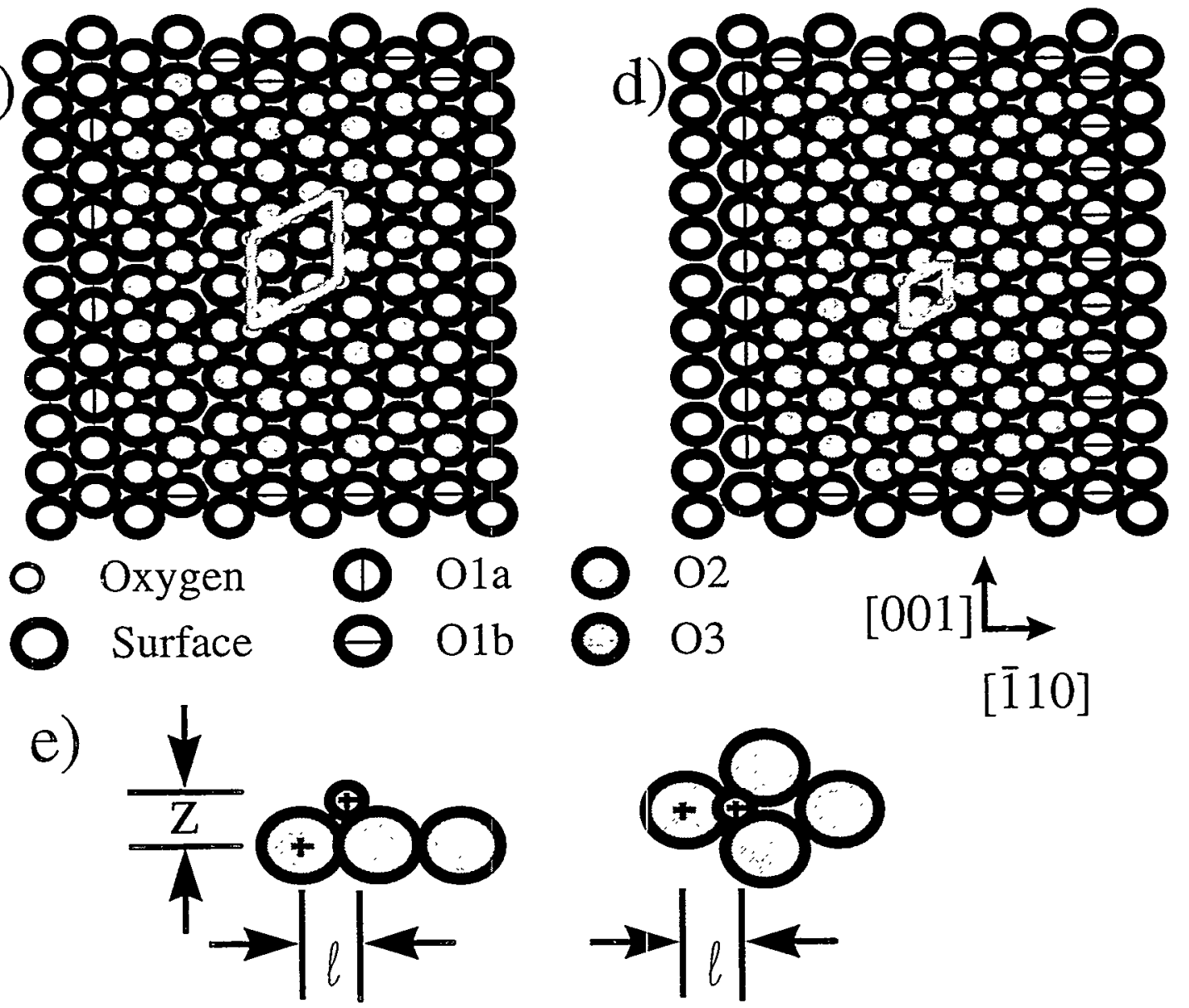

[110]

Figure 5.1. Illustration of different types of surface tungsten atom types with oxygen adsorption on W(110): a) Sparsely covered, b) p $(2 \times 1)$, c) p (2x2), and d) (1x1). "O1" denoted W bound to one oxygen atom, "O2" to two, and "O3" to three. (e) Nearestneighbor atomic geometry for oxygen adsorbed in a pseudo-threefold-hollow site. The parameters varied for the R-factor analysis are the height of the oxygen layer above the tungsten surface $(\mathrm{z})$ and the lateral displacement of oxygen as referenced from the ontop position $(\ell)$. 


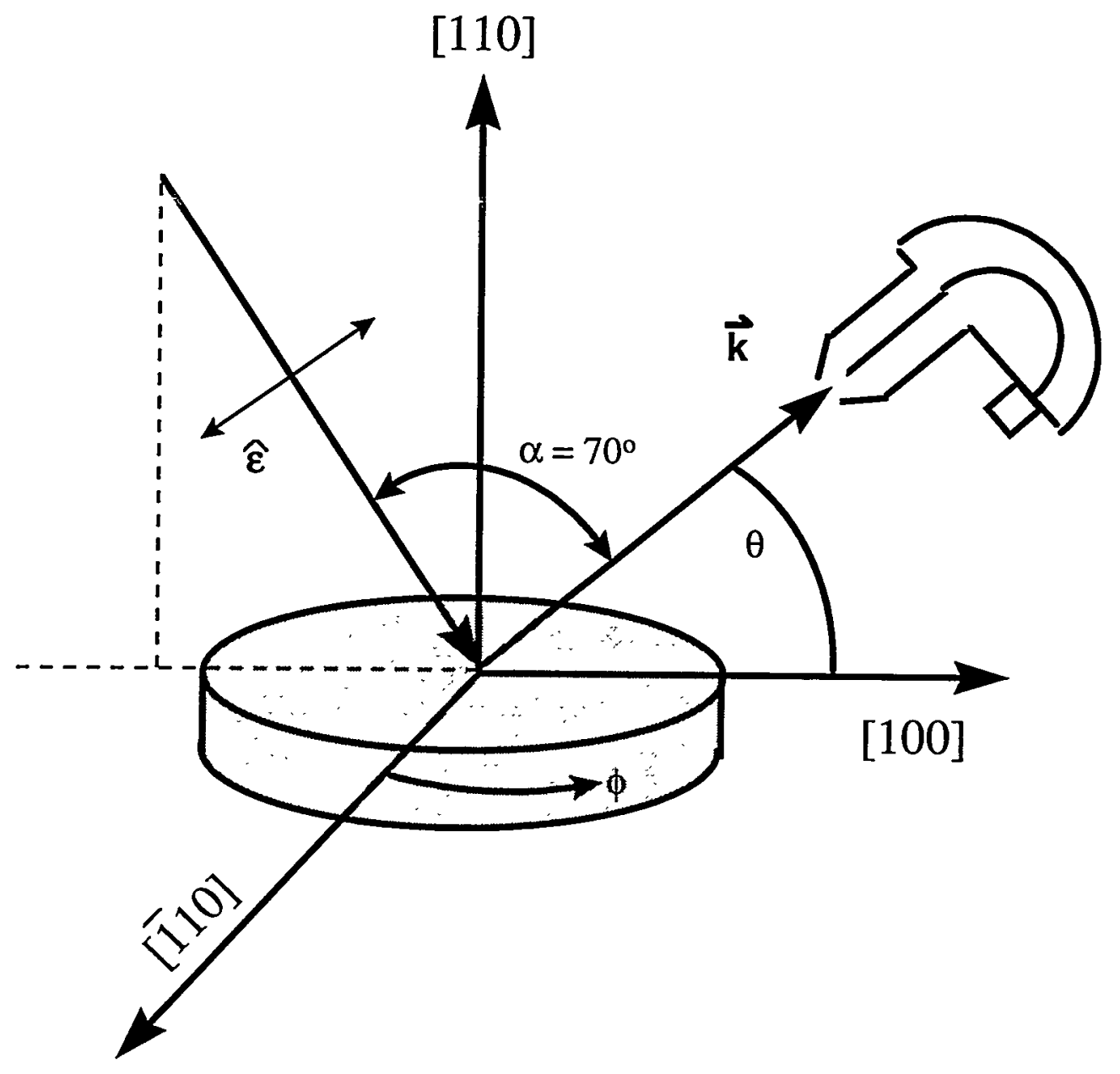

Figure 5.2. The experimental geometry. For the kinetics experiments, the angle $\theta$ was fixed at $45^{\circ}$. For the diffraction experiments, the angle $\theta$ was rotated from $9^{\circ}$ to $70^{\circ}$. Diffraction is measured by rotating the sample on the two perpendicular axes. 


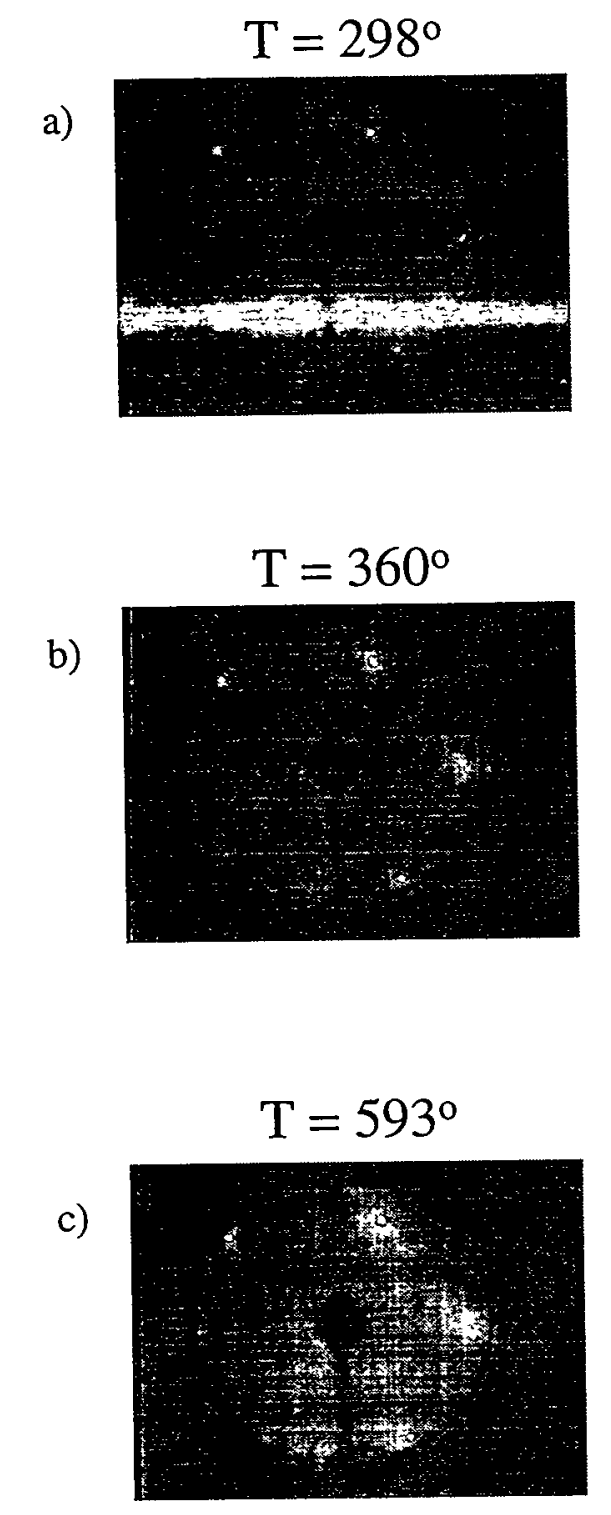

d)

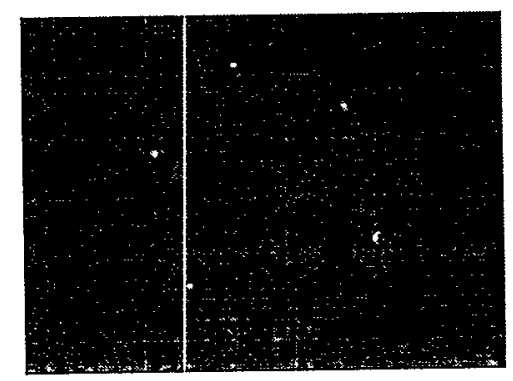

Clean W(110)

$0.00 \mathrm{ML}$

e)

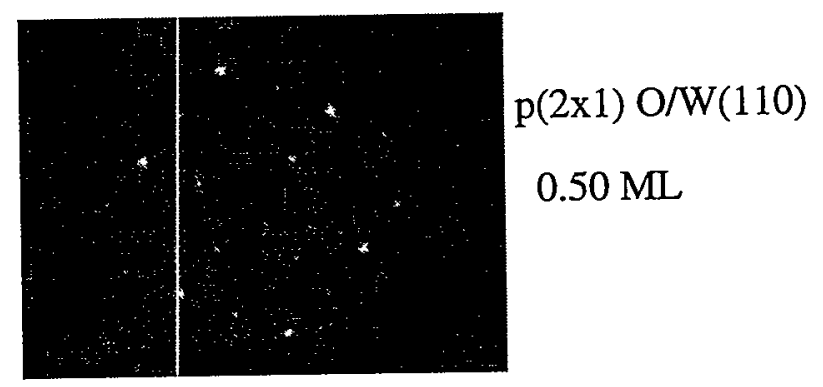

f)

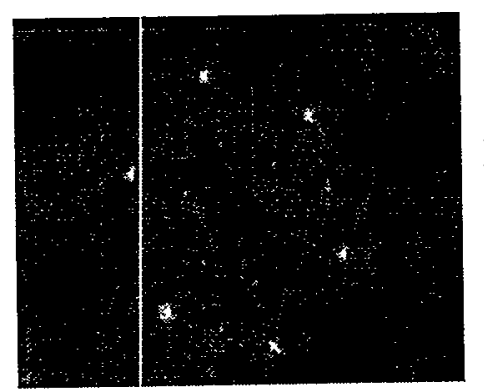

$\mathrm{p}(2 \times 2) \mathrm{O} / \mathrm{W}(110)$

$0.75 \mathrm{ML}$

g)

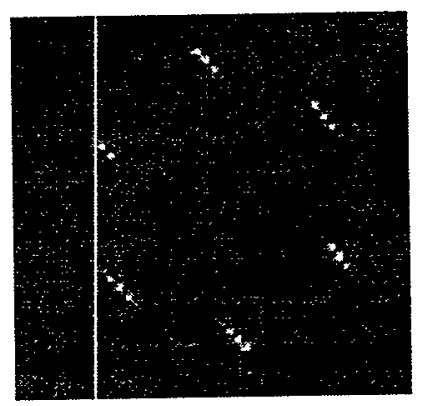

(1x1)(1x12) O/W(110)

$1.00 \mathrm{ML}$

Figure 5.3. a), b), c) -- LEED patterns on W[110] after 70 minutes of exposure to oxygen at $3.0 \times 10^{-9}$ Torr for sample temperatures of (a) $298^{\circ}$, (b) $360^{\circ}$, and (c) $593^{\circ}$, as measured with a primary energy of $85 \mathrm{eV}$. d), e), f), g) -- LEED patterns of common oxygen structures with long-range order on W[110], as measured with a primary energy of $93 \mathrm{eV}$ : (d) Clean W(110) surface, (e) $\mathrm{p}(2 \times 1)$ surface, (f) $\mathrm{p}(2 \times 2)$ surface, and $(\mathrm{g})(1 \times 1) \times 12$ surface. 


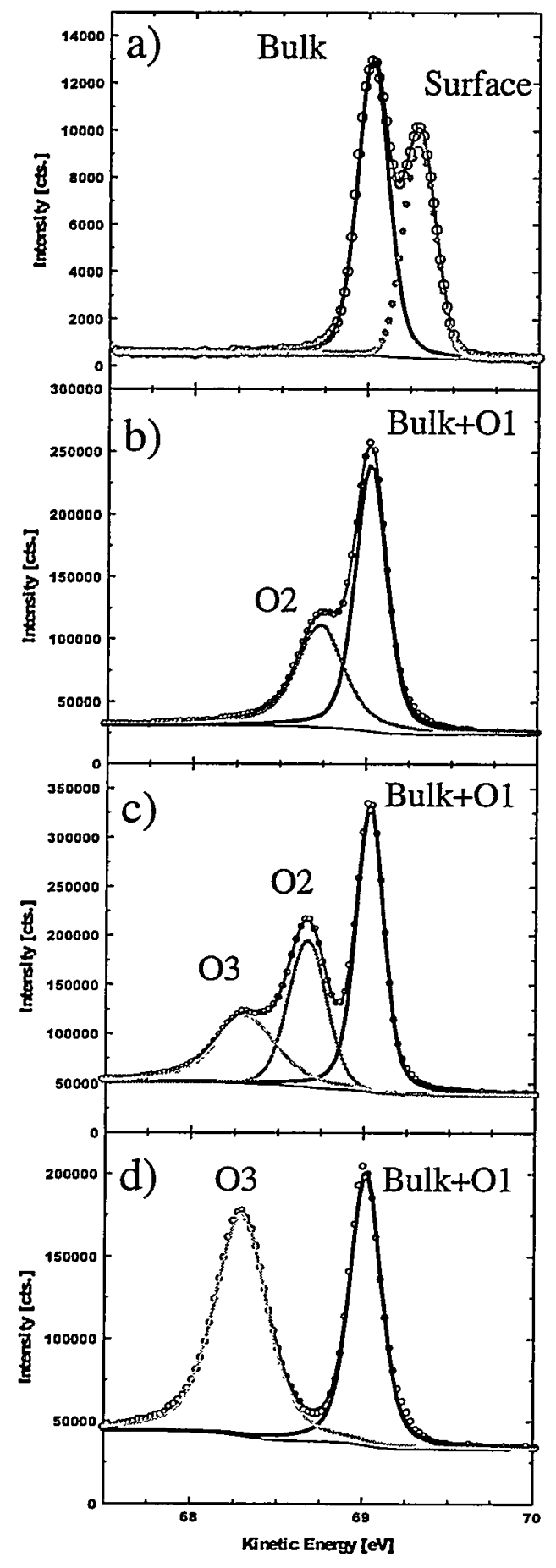

\author{
Clean W(110) \\ $0.00 \mathrm{ML}$
}

$\mathrm{p}(2 \times 1) \mathrm{O} / \mathrm{W}(110)$

$0.50 \mathrm{ML}$

$\mathrm{p}(2 \times 2) \mathrm{O} / \mathrm{W}(110)$

$0.75 \mathrm{ML}$

(1x1)-

(1x12) O/W(110)

$1.00 \mathrm{ML}$

.... Surface

- Bulk+O1

$\longrightarrow \mathrm{O} 2$

O3

Figure 5.4. W $4 \mathrm{f}_{7 / 2}$ Photoelectron spectra of common oxygen structures with longrange order on $\mathrm{W}[110]$ with internal photoelectron emission along the [111] direction, and an externally measured kinetic energy of $69 \mathrm{eV}$. (a) Clean W(110)

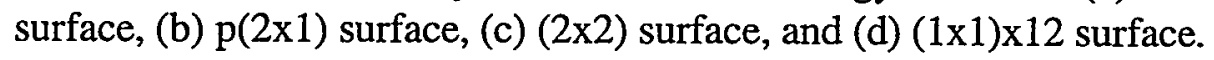



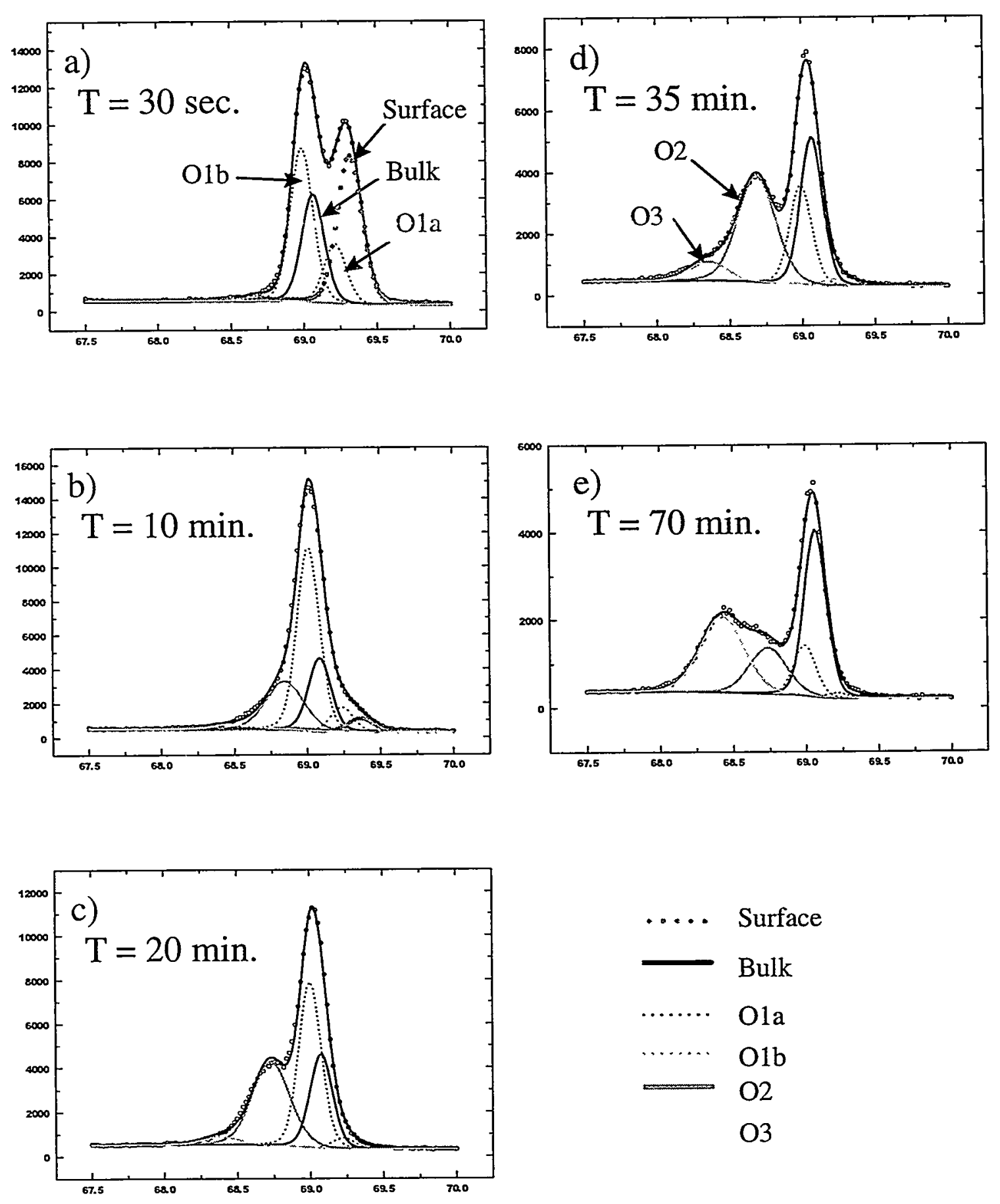

Figure 5.5. Time resolved $\mathrm{W} 4 \mathrm{f}_{7 / 2}$ spectra with internal photoelectron emission along the [111] direction, and an external kinetic energy of $69 \mathrm{eV}$. The spectra are taken at a) $30 \mathrm{sec}$., b) $10 \mathrm{~min}$., c) $20 \mathrm{~min}$., d) $35 \mathrm{~min}$., and e) $70 \mathrm{~min}$. of exposure to a pressure of $3.0 \times 10^{-9}$ torr $\mathrm{O}_{2}$. The spectra have been decomposed into six components as indicated, via curve fitting. 


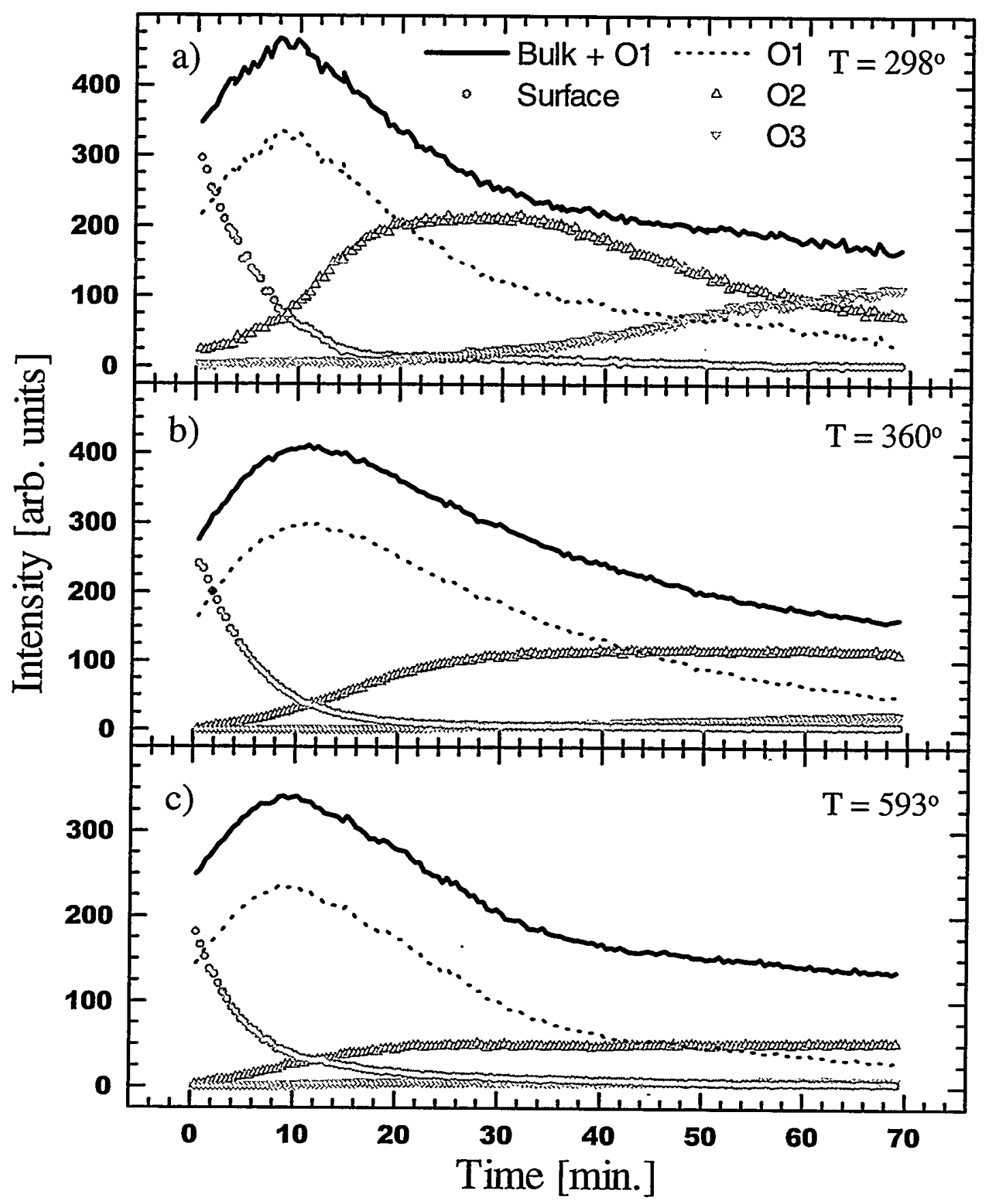

Figure 5.6. Time- and state- resolved $\mathrm{W} 4 \mathrm{f}_{7 / 2}$ photoelectron intensities for three diffeerent sample temperatures (a) $\mathrm{T}=298^{\circ}$, (b) $\mathrm{T}=360^{\circ}$, and (c) $\mathrm{T}=593^{\circ}$. The Surface, Bulk+O1, $\mathrm{O} 2$, and $\mathrm{O} 3$ intensities are directly measured. The $\mathrm{O} 1$ intensity is derived by assuming that the bulk intensity is constant with time and that the $\mathrm{Ol}$ is 0.4 of the $\mathrm{O} 2$ at the end of the scan (as derived from the two-domain Monte Carlo analysis). 


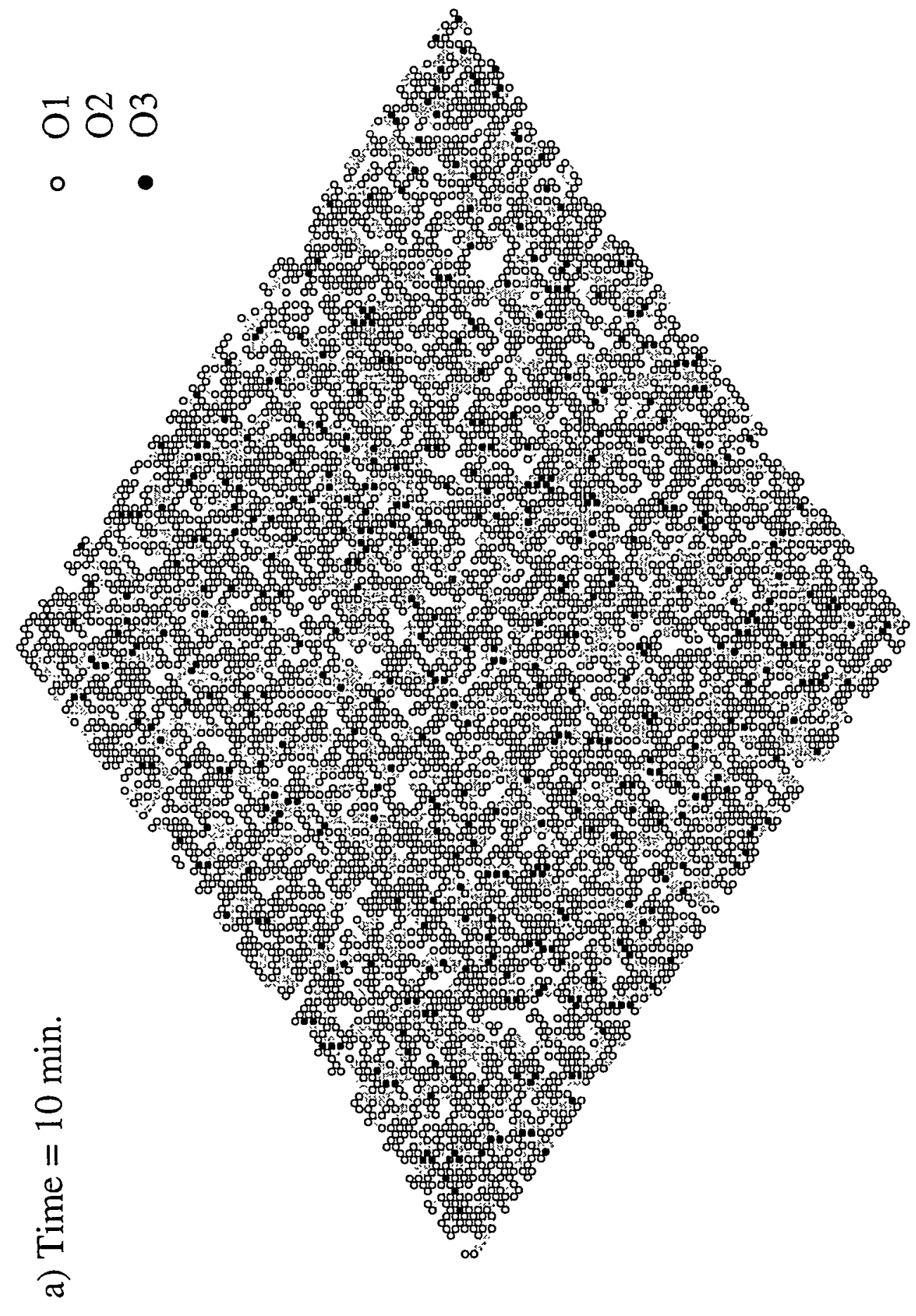

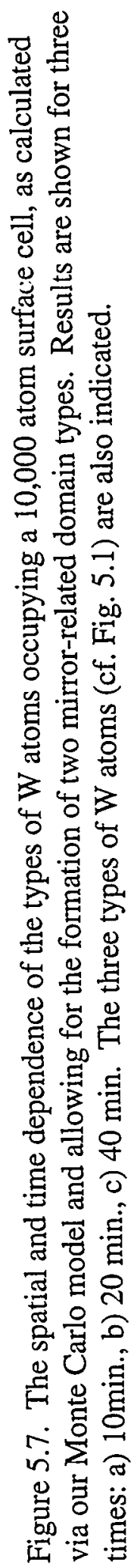




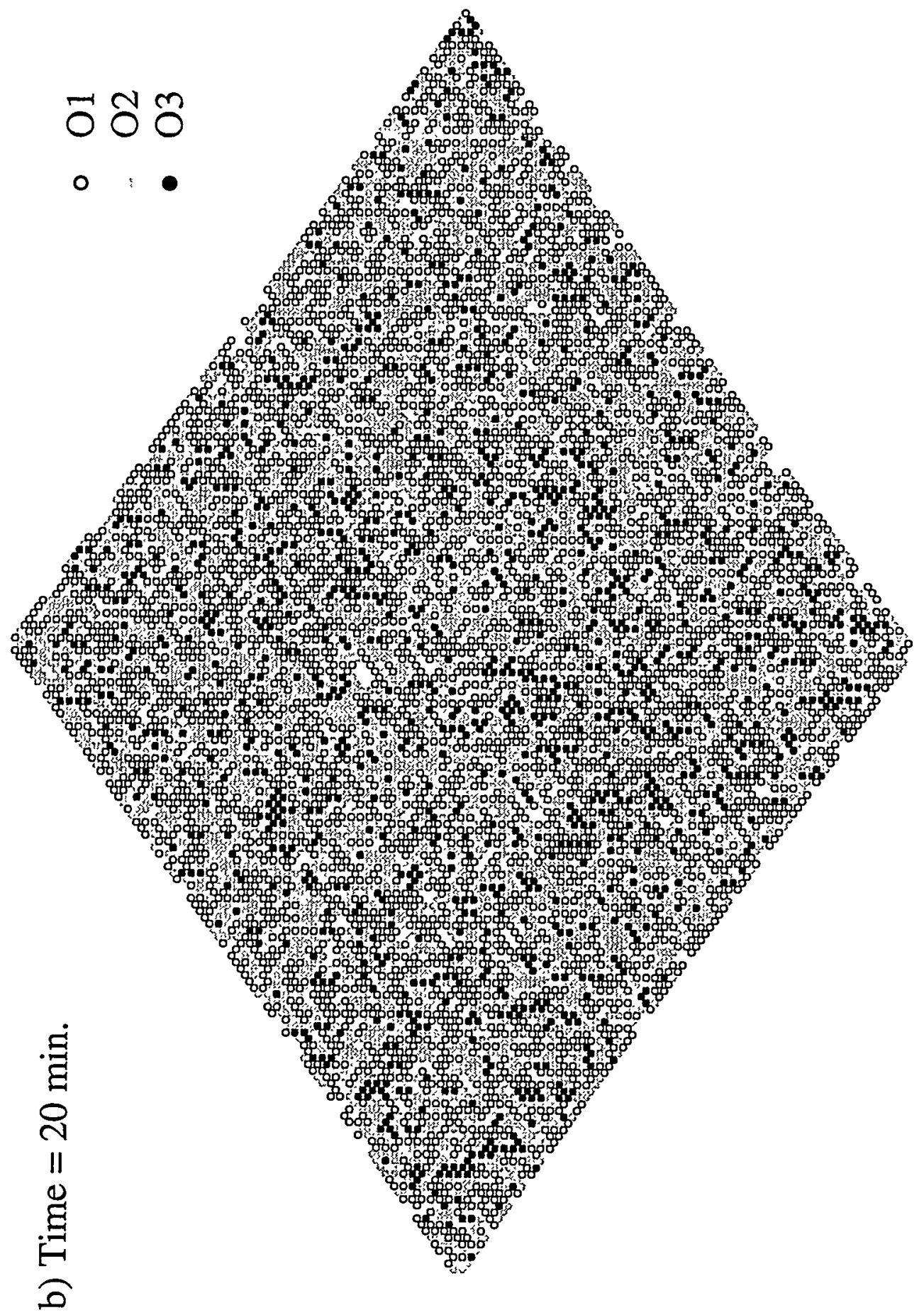




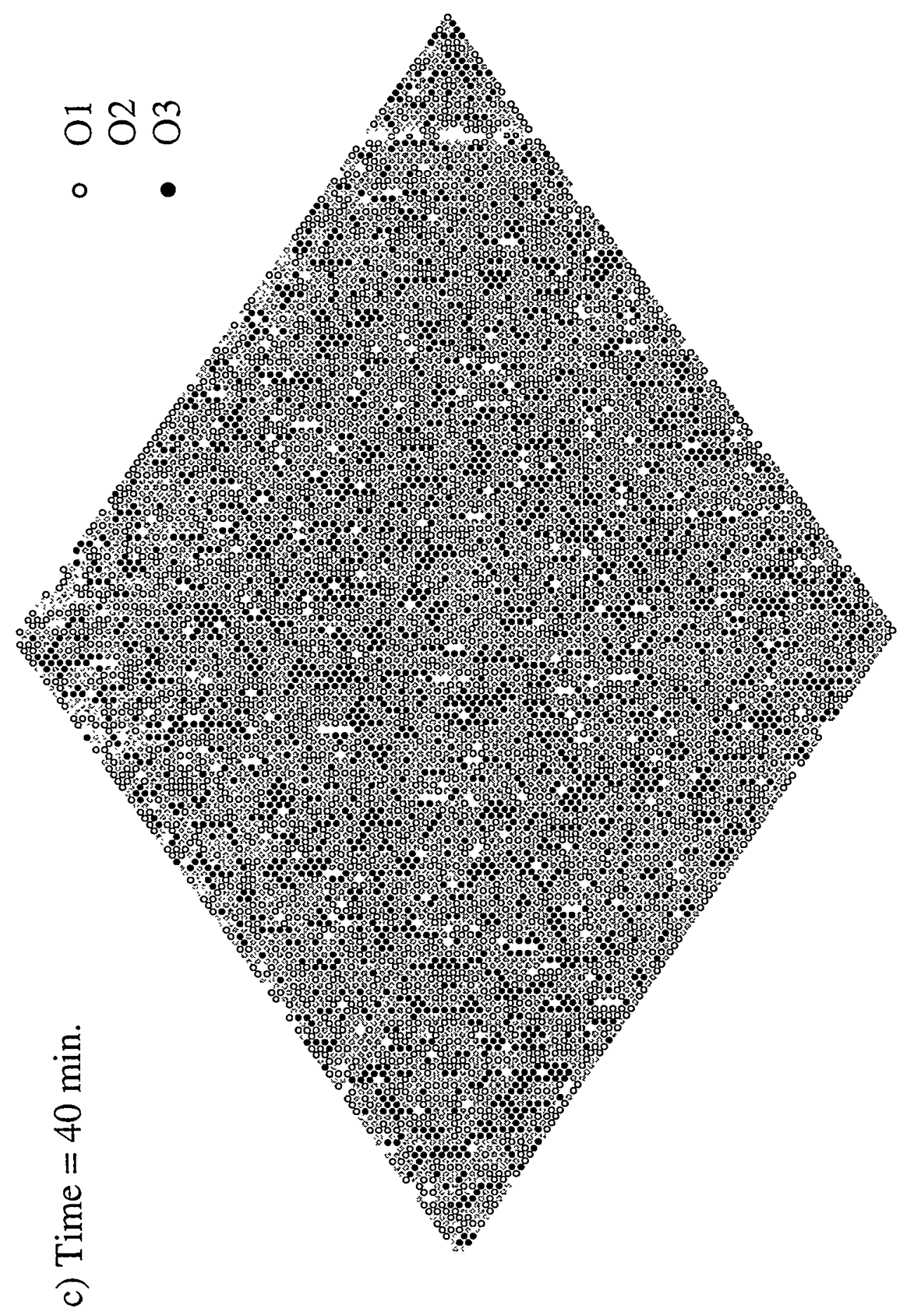




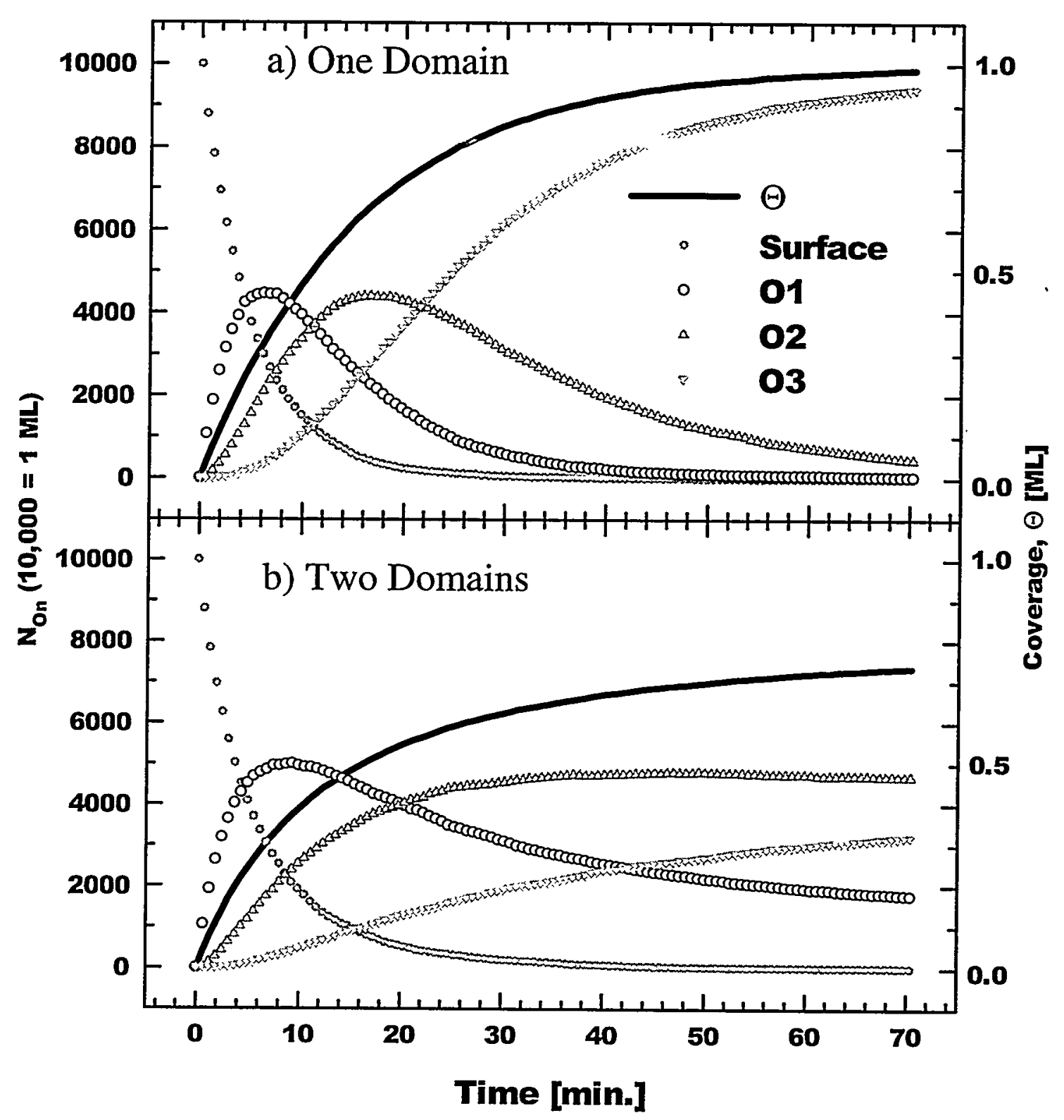

Figure 5.8. Monte Carlo simulation of oxygen adsorption on the W(110) surface for (a) one domain and (b) two domains. Surface, $\mathrm{O} 1, \mathrm{O} 2$, and $\mathrm{O} 3$ occupations $\mathrm{N}_{\mathrm{On}}$ and total oxygen coverage are plotted. 


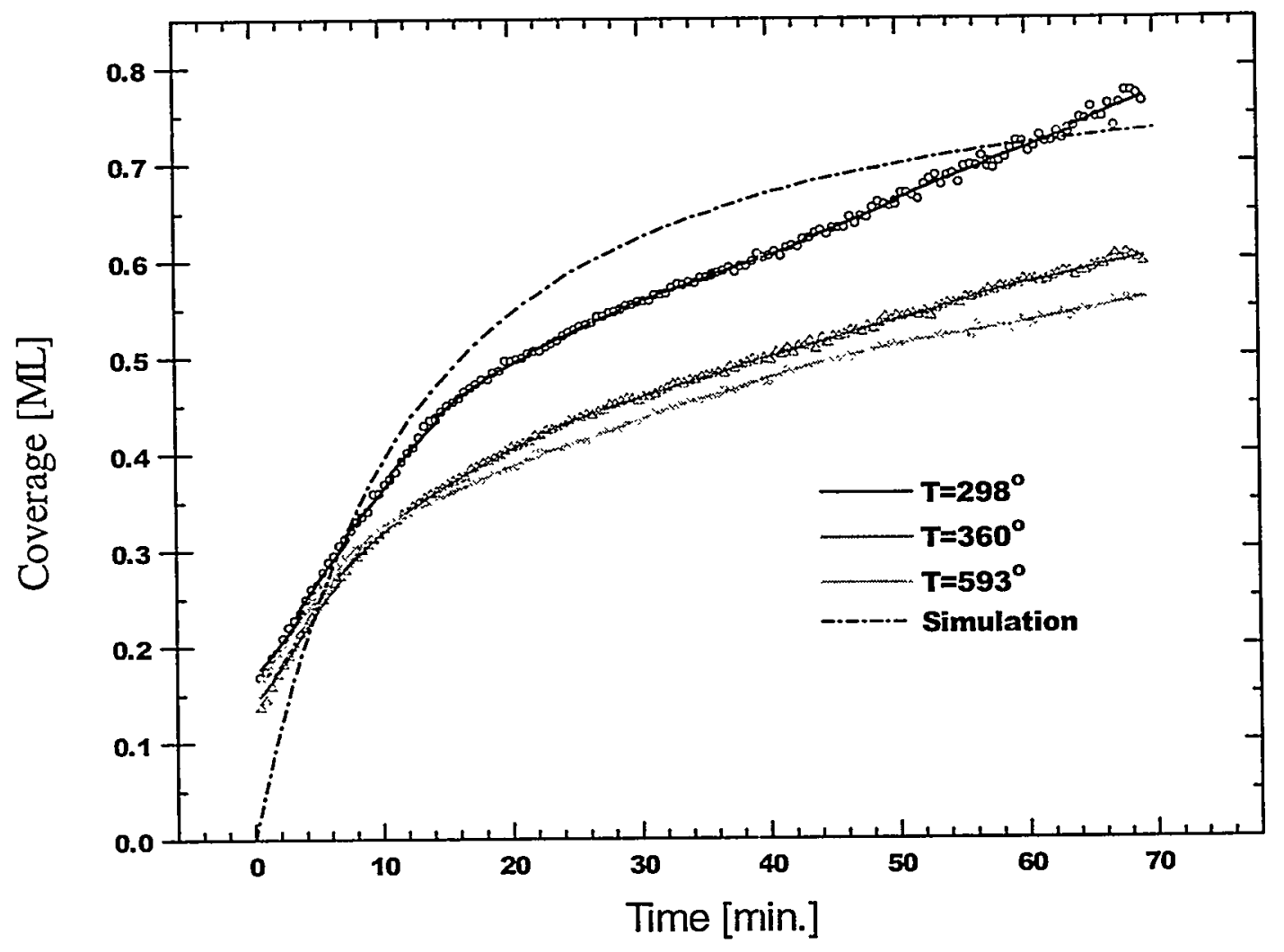

Figure 5.9. Time dependence of oxygen coverage: coverage calculations from timeand state-resolved $\mathrm{W} 4 \mathrm{f}_{7 / 2}$ intensities via Eq. 5.1 are shown as points. Associated lines are from 15-point smoothing. The dash-dot curve is a coverage calculation directly from the two-domain Monte Carlo simulation. 


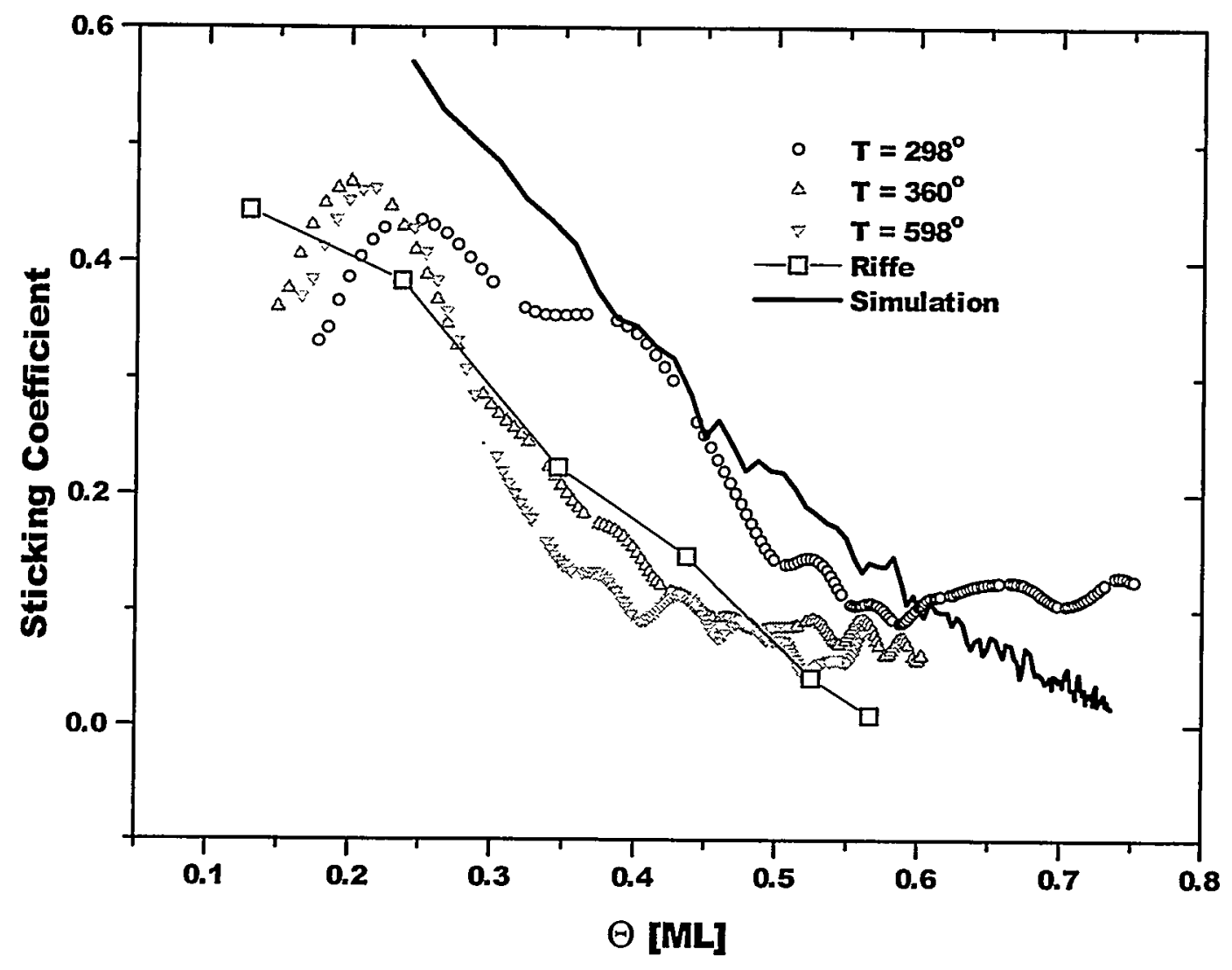

Figure 5.10. Oxygen sticking coefficients derived from the coverage data of Fig. 5.9. Symbols are the derivatives of the 15-point smoothed curves in Fig. 5.9, and the solid curve is from the Monte Carlo simulation. The square points are from a recent study by Riffe and Wertheim [14]. 


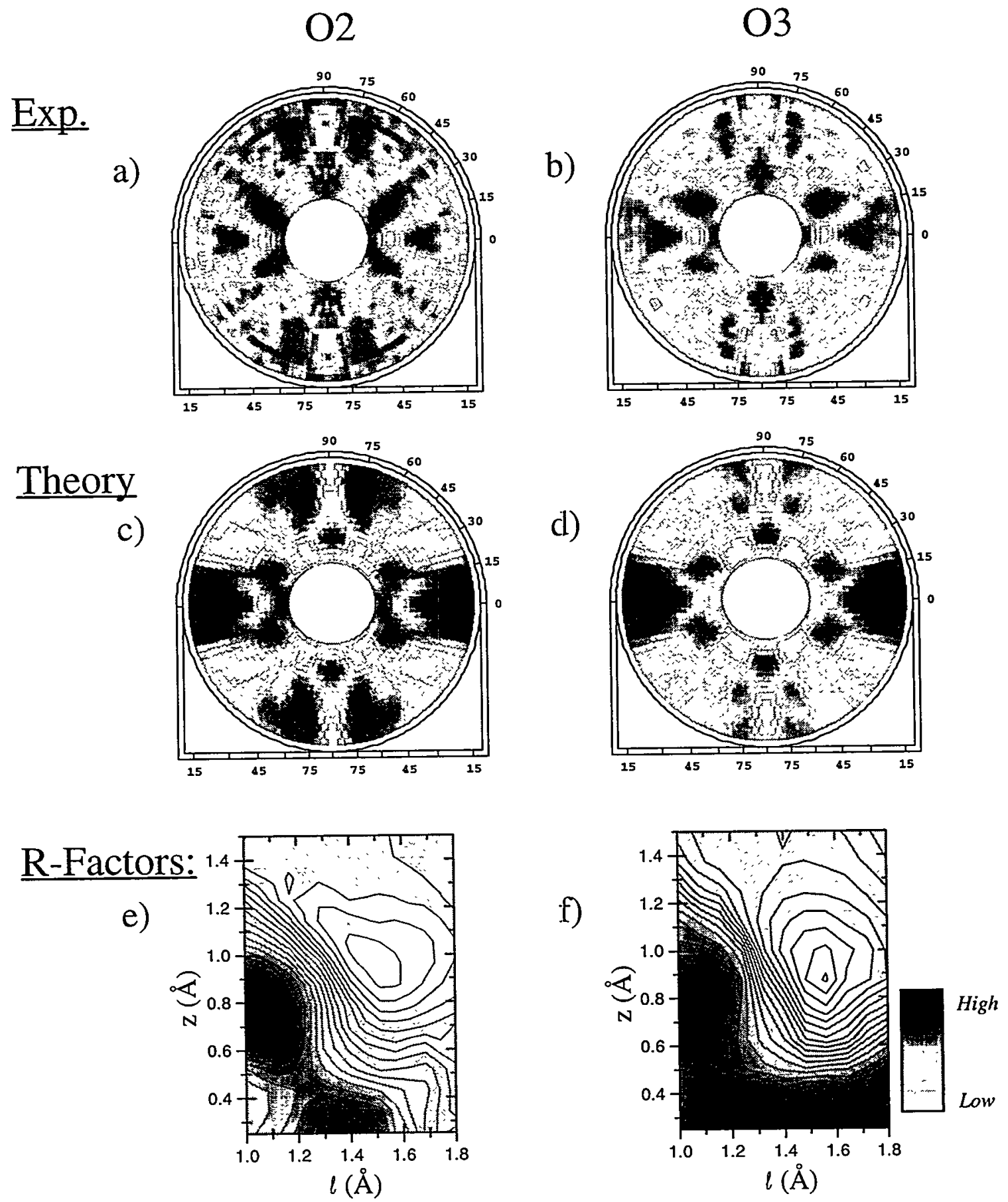

Figure 5.11. Surface structure determination from $\mathrm{W} 4 \mathrm{f}_{7 / 2}$ state-resolved photoelectron diffraction after 70 minutes exposure of oxygen at room temperature. Experimental diffraction data from the (a) $\mathrm{O} 2$ and (b) $\mathrm{O} 3$ species. Multiple scattering theory at the optimum structure for the (c) $\mathrm{O} 2$ and (d) $\mathrm{O} 3$ species. A normalized sum of five Rfactors as a function of the two structural parameters $z$ and $\ell$ is shown in e) and $f$ ). The optimum positions for the oxygen atoms are (e) $\mathrm{z}=0.97 \AA$ and $\ell=1.51 \AA$ for the $\mathrm{O} 2$ $\mathrm{W}$ sites, and (f) $\mathrm{z}=0.88 \AA$ and $\ell=1.56 \AA$ for the $\mathrm{O} 3 \mathrm{~W}$ sites. 


\section{Chapter 6}

\section{Circular Dichroism in Core Photoelectron Emission from (1x1) Oxygen on W(110): Experiment and Multiple-Scattering Theory}

\section{I. INTRODUCTION}

Circular dichroism in core-level excitation is now a very active area of study [15], due both to the availability of circularly polarized synchrotron radiation in the vacuum ultraviolet and x-ray regimes, and to the realization that such measurements can be useful as element-specific probes of magnetic materials [1,3], non-magnetic materials [3(d),4] and adsorbates [2]. Although circular dichroism has been observed in both core-level xray absorption spectroscopy (XAS) [1], and angle-resolved core-level photoelectron spectroscopy (PS) [2-4], we will here concentrate on the latter more surface-sensitive type of experiment in which the direction of the outgoing electron is precisely measured. To date, circular dichroism has been observed experimentally in angular distributions of core photoelectrons from both adsorbed molecules and atoms in single-crystal substrates [2-4]. It is also important to distinguish circular dichroism in non-magnetic systems $[2,4]$ from similar effects in magnetic systems [3]. In non-magnetic systems, chirality can be introduced into the experiment by particular choices of the photon-electron-substrate crystal geometry, with scattering of the photoelectrons from near-neighbor atoms in the crystal then playing a crucial role in affecting the angular distributions so as to lead to dichroism $[2,4,5]$. Although molecular quantum-chemical calculations of the final-state photoelectron wave functions were first used to predict such effects in non-magnetic 
cases [2(a)-(c)], photoelectron diffraction theory has also been shown to provide a general method for predicting and analyzing such effects for more complex systems [2(d),5]. In magnetic systems, one must consider in addition the effects of spin-orbit splitting and multiplet (exchange) splitting of the spectra, which provide additional contributions to dichroism [3]. However, the non-magnetic sources of dichroism will always be present, and it is thus necessary to allow for these fully in order to derive information specific to magnetic order. A principal aim of this paper is thus to more quantitatively assess these non-magnetic circular dichroism effects.

Such non-magnetic circular dichroism in core photoelectron angular distributions (CDAD) was first observed by Schönhense et al. from CO/Pd(111) [2(a)-(c)]. This experiment showed that circular dichroism may be present in core photoelectron emission, even in what are overall non-chiral atomic geometries. Subsequent to this, Daimon et al. observed similar effects in core emission from $\operatorname{Si}(100)$ [4(a)], and we will relate our experimental findings for $\mathrm{O} / \mathrm{W}$ to a theoretical model for these effects introduced by them, as well as to more accurate multiple scattering photoelectron diffraction calculations of such effects.

In order to better understand the interrelationship between such non-magnetic circular dichroism and magnetic circular dichroism, we have performed measurements for the non-magnetic system of a (1x1) saturated oxygen overlayer on $\mathrm{W}(110)$ using a thirdgeneration synchrotron radiation beamline and end station [6]. This incipient-oxide overlayer corresponds to a coverage of $1.0 \mathrm{ML}$ of oxygen, leads for our surface 
preparation to a (1x12) superstructure in its LEED pattern [8] that is similar to that observed in a prior STM study [7(a)], and exhibits W $4 \mathrm{f}$ core spectra that are easily resolvable into "incipient oxide" and bulk components separated by $\sim 0.73 \mathrm{eV}[7(\mathrm{~b}),(\mathrm{c}), 8]$. For convenience, we will term this structure $(1 \times 1) \times 12$ O/W(110), but the "oxide" W atoms in this surface are in fact locally in a $\mathrm{p}(1 \mathrm{xl}) \mathrm{O}$ chemisorption environment in which each $\mathrm{W}$ is bonded to three $\mathrm{O}$ atoms [7(a)]. This system has several advantages for the present study. It forms a very stable non-magnetic overlayer. Also, due to the presence of chemically-resolved spectra, we have distinguishable signals coming from two different types of tungsten atoms, those in the outermost $\mathrm{W}$ layer directly bonded to the oxygen atoms and those in the "bulk" layers below. Thus, in comparing experiment to theory, we have both the case of near-surface oxide emitters which look outward through only one layer of oxygen scatterers, and for which multiple scattering is probably less important, and multi-layer bulk emitters, for which multiple scattering effects are expected to be more important. Finally, the atomic structure of this surface has recently been determined accurately from scanned-angle photoelectron diffraction measurements $[7(b),(c), 8]$, providing accurate structural parameters to use in our theoretical simulations.

\section{II. EXPERIMENT}

One of the several advantages of using synchrotron radiation for such a study is the possibility of varying the polarization from p-type linear (here denoted $L P_{x}$ ) to left and right circular (LCP and RCP, respectively), and we have used bend-magnet beamline 9.3.2 at the Advanced Light Source [6] to exploit this capability in high-resolution core- 
level photoelectron emission. This beamline is capable of varying polarization over a broad energy range from $\sim 30$ to $1500 \mathrm{eV}$ by using radiation in, above, or below the plane of the electron storage ring. In connection with core photoelectron emission from surfaces, it then becomes possible to look for circular dichroism effects in which the intensity with LCP excitation ( $\mathrm{I}_{\mathrm{LCP}}$ ) is not equal to the intensity with $\mathrm{RCP}$ excitation ( $\mathrm{I}_{\mathrm{RCP}}$ ), and to compare these two types of measurements with those involving $\mathrm{LP}_{\mathrm{X}}$ excitation $\left(I_{L P x}\right)$. Angle-resolved and chemical-state-resolved photoelectron spectra were measured using the advanced photoelectron spectrometer/diffractometer situated on this beamline [3(d)]. The $(1 \times 1) \times 12$ oxygen overlayer was prepared on the clean $W(110)$ surface as described elsewhere $[7,8]$.

The experimental geometry is shown in Fig. 6.1(a). The photons were incident normal to the sample surface and chosen to be linearly polarized in the plane of the storage ring (p-polarized with respect to the plane of light incidence and electron exit or $x$ in our convention), left circularly polarized, or right circularly polarized. The polar takeoff angle theta $(\theta)$ is measured with respect to the surface, and the azimuthal angle phi $(\phi)$ is measured with respect to the [1 $\overline{1} 0]$ direction lying in the surface. An $85 \%$ degree of circular polarization was achieved by means of a movable aperture at the entrance to the beamline; the intensity loss on going from linear to circular was only about a factor of $1 / 3-1 / 4$ [6]. The experiment was conducted at a photon energy of 280 $\mathrm{eV}$, leading to $\mathrm{W}$ 4f photoelectrons with a kinetic energy of $246-250 \mathrm{eV}$. This corresponds to one of the kinetic energies used by Daimon et al. in a previous study of circular dichroism in $\mathrm{Si} 2 \mathrm{~s}$ and $2 \mathrm{p}$ emission from silicon [4], permitting a more direct 
comparison of these two sets of CDAD data. The photoelectrons were analyzed using a Scienta SES 200 spectrometer [3(d)]. A typical spectrum is shown in Fig. 6.1(b), this spectrum is taken with $\theta=44^{\circ}$ and $\phi$ pointing along the $\mathrm{W}<001>$ azimuthal direction in the sample plane (i.e. $\phi=90^{\circ}$ ). The $\mathrm{W} 4 \mathrm{f}_{7 / 2}$ bulk peak occurs at a kinetic energy of 249.5 $\mathrm{eV}$, while the $\mathrm{W} 4 \mathrm{f}_{5 / 2}$ bulk peak is shifted to higher binding energy by $+2.25 \mathrm{eV}$. Each member of the W $4 \mathrm{f}_{5 / 2,7 / 2}$ doublet consists of a bulk peak and an oxide peak that is shifted to higher binding energy (lower kinetic energy) by $\sim+0.73 \mathrm{eV}$. Separate photoelectron diffraction patterns were measured for these two types of photoelectron peaks by fitting appropriate peak shapes to the spectra; the spectra were easily resolved into two components by fitting them with asymmetric Voigt functions of fixed width and spacing, riding on a Shirley-type inelastic background. Total $4 \mathrm{f}$ intensities for both oxide and bulk were then derived as the sum of the $5 / 2$ and $7 / 2$ areas for each type of peak. Summing in this way increases the number of counts and statistical reliability, and also allows us to neglect any effects due to spin-orbit coupling in the modeling of the data. The sample was rotated in azimuthal angle $(\phi)$ over $230^{\circ}$ and the analyzer was rotated in polar (takeoff) angle ( $\theta$ ) over a range of approximately $27.5^{\circ}$ so as to preserve the normal incidence of the light. In this way, both intensities could be measured over a $\theta$ range (as measured with respect to the surface plane) of $16.5^{\circ}$ to $44^{\circ}$ and a solid angle corresponding to $\sim 41 \%$ of the $2 \pi$ steradians above the surface. The analyzer rotation was achieved through the use of differentially pumped seals at the top and bottom of the main experimental chamber, and a large diameter bellows that allowed the light to pass from the last refocusing mirror of the beamline to the sample position [3(d),8]. Any variations of radiation flux with time were normalized out in determining the final intensities $\mathrm{I}(\theta, \phi)$. 


\section{III. THEORETICAL BACKGROUND}

When the light propagation direction $\mathbf{q}$ is along the positive $\mathrm{z}$ axis, RCP (LCP) light can be represented by the operator $\mathbf{x}$-iy $(\mathbf{x}+\mathbf{i} \mathbf{y})$, where $\mathbf{x}$ and $\mathbf{y}$ are polarization vectors along the $\mathrm{x}$ and $\mathrm{y}$ axes. In this definition, $\mathrm{RCP}$ (LCP) rotates in a clockwise (counterclockwise) direction as viewed by an observer looking into the oncoming light from positive $\mathrm{z}$ and has a negative (positive) helicity. Linearly polarized light of $\mathrm{p}$-type $\left(L P_{x}\right)$ and linearly-polarized light of s-type $\left(L P_{y}\right)$ along the $x(y)$ direction can then be represented by the operators $\mathbf{x}(\mathbf{y})$.

A brief description of our calculational procedure as applied first to a free atom, and then to an atom in a lattice, is given below. The photoelectron intensity due to excitation from a given initial state $\phi_{n l m}$ to a final state $\phi_{\mathrm{f}}$ (which can in general involve scattering and diffraction in a lattice) due to LCP or RCP light can be written in a standard way as

$$
\mathrm{I}_{\mathrm{LCP}(\mathrm{RCP})}=\left|\left\langle\phi_{\mathrm{f}}|\mathbf{x} \pm \mathbf{i} \mathbf{y}| \phi_{n l m}\right\rangle\right|^{2}=\left|\left\langle\phi_{\mathrm{f}}|\mathbf{x}| \phi_{n l m}\right\rangle \pm \mathrm{i}\left\langle\phi_{\mathrm{f}}|\mathbf{y}| \phi_{n l m}\right\rangle\right|^{2}
$$

For $\mathrm{LP}_{\mathrm{x}}$ or $\mathrm{LP}_{\mathrm{y}}$ light, we also have the photoelectron intensities as

$$
I_{\mathrm{LPx}}=\left|\left\langle\phi_{\mathrm{f}}|\mathbf{x}| \phi_{n l m}\right\rangle\right|^{2}
$$


and

$$
\mathrm{I}_{\mathrm{LPy}}=\left|\left\langle\phi_{\mathrm{f}}|\mathbf{y}| \phi_{n l m}\right\rangle\right|^{2}
$$

Finally, as a reference for unpolarized light we have,

$$
\mathrm{I}_{\mathrm{UNP}}=\left|\left\langle\phi_{\mathrm{f}}|\mathrm{x}| \phi_{n l m}\right\rangle\right|^{2}+\left|\left\langle\phi_{\mathrm{f}}|\mathbf{y}| \phi_{n l m}\right\rangle\right|^{2}=\mathrm{I}_{\mathrm{LPx}}+\mathrm{I}_{\mathrm{LPy}}=\mathrm{I}_{\mathrm{RCP}}+\mathrm{I}_{\mathrm{LCP}}
$$

Using these equations, we can rewrite the result for circularly polarized light as

$$
I_{L C P(R C P)}=I_{L P x}+I_{L P y} \pm 2 \sqrt{I_{L P x} I_{L P y}} \sin \Delta
$$

where $\Delta$ is the phase between the two integrals $\left\langle\phi_{\mathrm{f}}|x| \phi_{n l m}\right\rangle$ and $\left\langle\phi_{\mathrm{f}}|\mathrm{y}| \phi_{n l m}\right\rangle^{*}$. In calculating the matrix elements for a free atom, the initial core state is written in the nonrelativistic limit as

$\phi_{n l m}(\mathrm{r}, \theta, \phi)=\mathrm{R}_{n l}(\mathrm{r}) \mathrm{Y}_{l m}(\theta, \phi)$

where $\mathrm{R}_{n l}(\mathrm{r})$ is the radial part of the wavefunction, $\mathrm{Y}_{l m}(\theta, \phi)$ is a spherical harmonic, and the final photoelectron state $\phi_{\mathrm{f}}$ at energy $E_{\mathrm{kin}}$ and propagating along the wave vector $\mathbf{k}$, in the absence of final-state scattering, is written as 


$$
\begin{gathered}
\phi_{\mathrm{f}}(\mathrm{r}, \theta, \phi)=4 \pi \sum_{l^{\prime} m^{\prime}} i^{l^{\prime}} \exp \left(-i \delta_{l^{\prime}}\right) \mathrm{Y}_{l^{\prime} m^{\prime}}^{*}\left(\theta_{k}, \phi_{k}\right) \mathrm{Y}_{l^{\prime} m^{\prime}}(\theta, \phi) \mathrm{R}_{\mathrm{r}, l^{\prime}}(\mathrm{r}) \\
=4 \pi \sum_{l^{\prime} m^{\prime}} \phi_{\mathrm{f}, l^{\prime} m^{\prime}}(\mathrm{r}, \theta, \phi)
\end{gathered}
$$

where $\delta_{l}$, is the radial phase shift for the $l^{\prime}$ final state channel, $\mathrm{R}_{\mathrm{f}, l^{\prime}}(\mathrm{r})$ is the radial part of the $l^{\prime}$ channel, and $\phi_{f, l} m^{\prime}$ is introduced to simplify the notation. We now take the $z$-axis to be along $\mathbf{q}$, with the spherical harmonics for both the initial and final states then being quantized along that axis as well. The total intensity from a given subshell is then given by

$$
I_{n, l, L C P(R C P)}=\sum_{m}\left|\sum_{l^{\prime} m^{\prime}}\left[\left\langle\phi_{f, l^{\prime} m^{\prime}}|x| \phi_{n l m}\right\rangle \pm \mathrm{i}\left\langle\phi_{f, l^{\prime} m^{\prime}}|y| \phi_{n l m}\right\rangle\right]\right|^{2}
$$

The first sum is over the initial occupied $\mathrm{m}$ states, and the second is over the final $l^{\prime} m^{\prime}$ states as allowed by dipole selection rules. An analogous sum applies to $\mathrm{I}_{n l}$ for LPx, LPy, and UNP. Final-state interference between the $l^{\prime}=l+1$ and $l^{\prime}=l-1$ channels is thus explicitly included.

The generalization from the free-atom final-state wave function so as to include a full multiple-scattering treatment of photoelectron scattering and diffraction effects in a cluster of atoms is found elsewhere [5,9]. Briefly, the photoelectron wave function for this case can be written by noting that each neighboring atom " $\mathrm{a}$ " in a cluster around the emitter acts as a scattering center, and can be considered to generate a spherical outgoing wave contribution to the final photoelectron wave, which is finally measured in a far-field 
detector. This outgoing wave furthermore includes contributions from all single and multiple scattering paths between the emitter and atom "a". With these assumptions, the photoelectron wave component $\phi_{\mathrm{f}, l^{\prime} m^{\prime}}$ can be written as a sum overall all atomic positions $r_{\mathrm{a}}$ as:

$\phi_{\mathrm{f}, l^{\prime} m^{\prime}}(\mathrm{r}, \theta, \phi)=\sum_{\mathrm{a}} \mathrm{e}^{-\mathrm{i} \mathbf{k} \cdot \mathrm{r}_{\mathrm{a}}} \mathrm{C}_{\mathrm{a}, l^{\prime} m^{\prime}}^{f}(\mathrm{k}) \mathrm{Y}_{l^{\prime} m^{\prime}}(\theta, \phi) \mathrm{R}_{\mathrm{fa}, l^{\prime} m^{\prime}}(\mathrm{r})$

where $\mathrm{C}_{\mathrm{a}, r^{\prime}}^{\mathrm{f}}(\mathbf{k})$ includes all effects of scattering between the emitter and atom "a", and $\mathrm{R}_{\mathrm{fa}, l_{m}^{\prime}}(\mathrm{r})$ is the radial part of the partial wave $l^{\prime}, m^{\prime}$ as calculated in the potential of atom "a" at the photoelectron energy. This reduces to the free-atom limit when "a" $=0$ only for the emitter, in which case $\mathrm{C}_{0, l^{\prime} m}^{\mathrm{f}}(\mathbf{k})$ is directly derivable from Eq. 6.7. In the far-field limit, the radial functions in both the free-atom and cluster cases can be represented by spherical Hankel functions $h_{1}^{(+)}(k r)$. We have carried out calculations of the $\mathrm{C}_{\mathrm{a}, \ell^{\prime} m}^{\mathrm{f}}(\mathbf{k})$ using both the Rehr-Albers separable Green's function approach to each scattering event [9(a),(b)], and the exact multiple scattering propagator [9(c)], although all results reported here will be based on the exact propagator.

As noted above, it is not necessary here to include spin-orbit coupling, as we will consider total subshell intensities as summed over the $5 / 2$ and $7 / 2$ peaks, although the general-purpose program we have used $[9(c)]$ in fact does incorporate spin-orbit effects and permits calculating such quantities as spin-orbit branching ratios. This program [9(c)] also allows for full multiple scattering of the photoelectron wave function as it exits the crystal (that is, $\phi_{\mathrm{f}}$ chhas the much more complicated form of Eq. 6.8 , as 
compared to the simple form given by Eq. 6.7) and includes effects of inelastic scattering (via an inelastic attenuation length $\Lambda_{\varepsilon}$ ), vibrational damping of diffraction (via DebyeWaller factors and an assumed Debye temperature $\Theta_{\mathrm{D}}$ ), and surface refraction of the photoelectrons in crossing the surface potential barrier (via an inner potential $\mathrm{V}_{\mathrm{o}}$ ). For the calculations reported below, a cluster of $30 \mathrm{O}$ atoms and $70 \mathrm{~W}$ atoms was used, with the oxygen assumed to be sitting in a pseudo-threefold hollow site at a vertical distance of $0.91 \AA$ and a lateral displacement of $0.10 \AA$ along the $[1 \overline{1} 0]$ direction and away from the point of three equal bond lengths, as determined from recent scanned-angle photoelectron diffraction studies $[7(b),(c), 8]$. The non-structural parameters used were $\Lambda_{\mathrm{e}}=6.5 \AA, \Theta_{\mathrm{D}}$ $=400 \mathrm{~K}$, and $\mathrm{V}_{\mathrm{O}}=11 \mathrm{eV}$, as derived from this prior work.

\section{IV. RESULTS AND DISCUSSION}

Azimuthal photoelectron diffraction patterns at polar takeoff angles $\theta$ of $16.5^{\circ}$, $26.5^{\circ}$, and $44.0^{\circ}$ are shown in Figs. 6.2, 6.3, and 6.4, respectively, for oxide emission and in Figs. 6.5, 6.6, and 6.7, respectively, for bulk emission. These three patterns are thus part of the larger data set, and represent the minimum, average, and maximum takeoff angles studied. These figures show experiment and theory for intensities with all three polarizations, (a) $\mathrm{LP}_{\mathrm{X}}$, (b) LCP, and (c) RCP, together with (d) the normalized circular dichroism in angular distributions (CDAD), which is defined in \% as $100\left[I_{R C P}\right.$ $\left.\mathrm{I}_{\mathrm{LCP}}\right] /\left[\mathrm{I}_{\mathrm{RCP}}+\mathrm{I}_{\mathrm{LCP}}\right]$. The azimuthal diffraction patterns have been normalized in intensity into a chi function to allow for direct comparisons of experiment with theory and more direct comparison of experiment for different polar angles. This normalization was done 
by first taking azimuthal averages of intensities at each polar angle, and then fitting the entire average data set with a low-order spline function in $\theta$ and treating this result as the intensity $I_{0}(\theta)$ in the absence of any diffraction; the resulting chi function is then calculated as $\chi(\theta, \phi)=\left[I(\theta, \phi)-\mathrm{I}_{0}(\theta)\right] / \mathrm{I}_{0}(\theta)$. We will defer discussing the theoretical results shown as grey curves in Figs. 6.2-6.7 for now, but return to them below.

As a self-consistency check on the experimental intensity data, we show in Figs. 6.8 and 6.9 the RCP intensities at the three polar angles in Figs. 6.2-6.7 overlaid with the mirror image of the corresponding LCP intensities. Due to the symmetry of the experimental geometry (cf. Fig. 6.1(a)), these overlaid curves should be identical, and we see that they indeed are to a very high degree, with the two bulk curves being almost superimposable in Fig. 6.9. The small differences between these two sets of curves then provide an indication of the overall statistical and systematic accuracies of the data.

Now comparing the experimental diffraction patterns for LCP and RCP excitation for either oxide or bulk in Figs. 6.2-6.7, we see that some features appear to exhibit azimuthal peak "rotations" across the mirror planes at $\phi=90^{\circ}$ and 180 , with the diffraction peaks for LCP being shifted to the left and those for RCP being shifted to the right. These kinds of rotations have been discussed previously by Daimon et al. [4], and we return to consider them in a more quantitative way below. However, other differences between the LCP and RCP curves do not follow such a simple characterization, and the full description of the data will be seen to require a multiple-scattering theory of photoelectron diffraction. There are also large dichroism effects, with the oxide showing 
up to $47 \%$ effects and the bulk showing up to $44 \%$ effects, as judged by the extrema over our entire data set.

Figs. 6.10(a)-(c) and 6.11(a)-(c) now show stereographic projections of the full experimental data sets for both oxide and bulk emission, respectively. Similar azimuthal peak rotations (especially visible in the oxide data) and other more complex changes between LCP and RCP can be seen here as well.

Diffraction peak rotations with changing circular polarization were first observed in $\mathrm{Si} 2 \mathrm{~s}$ and $2 \mathrm{p}$ emission from $\mathrm{Si}(001)$ and theoretically interpreted in terms of a physically intuitive model by Daimon et al. [4(a)]. This model relies on the dipole selection rules for circularly polarized light $\left(\Delta \mathrm{m}=\mathrm{m}^{\prime}-\mathrm{m}=-1\right.$ for $\mathrm{LCP}$ and $\Delta \mathrm{m}=+1$ for $\mathrm{RCP}$ ), combined with an estimate of the relative strengths of the various allowed

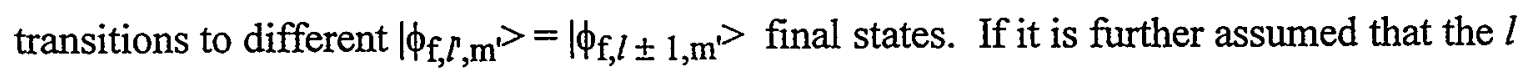
+1 channel dominates due to a much larger radial matrix element, this leads to final-state wave functions in a free-atom picture that are for many cases dominated by $\left|\phi_{\mathrm{f}, l, \mathrm{~m}^{\prime}}\right\rangle=\mid \phi$ $\mathrm{f}, l+1, \pm|l+1|>$, with the two signs holding for the two different circular polarizations. The spherical harmonics associated with these wave functions (cf. Eq. 6.7) in turn have an azimuthal dependence of $\exp \left(\mathrm{m}^{\prime} \phi\right)$, and they thus yield spiraling surfaces of constant phase in azimuth, rather than the simpler spherical surfaces of constant phase that are more often seen in the radial dependence of wave functions. Since the photoelectron current will be carried perpendicular to these constant phase surfaces, a given photoelectron diffraction feature (for example, associated with forward scattering along 
an interatomic direction) is predicted to be rotated in opposite directions by the two polarizations. A simple formula for estimating these rotations using the above assumptions, together with a final assumption that there is some kind of intensity peak due to scattering in the forward direction with which to observe the rotation, is the following:

$$
\Delta \phi=\frac{\mathrm{m}^{\prime}}{\mathrm{R}_{\mathrm{nn}, \|} k \cos \theta_{\mathrm{k}}}
$$

where $\Delta \phi$ is the azimuthal peak shift from the unperturbed forward scattering direction, $\mathrm{m}^{\prime}$ is the final-state $\mathrm{m}$ value, $\mathrm{R}_{\mathrm{nn}, \|}$ is the component of the nearest-neighbor distance along a certain forward scattering direction parallel to the surface, $k$ is the magnitude of the electron wave vector, and $\theta_{\mathrm{k}}$ is the angle between the surface and this forward scattering direction. This formula has been presented elsewhere and compared to experimental data $[4,10]$. and it is found to predict the changes in position of some, but not all, peaks in going from LCP to RCP excitation. For example, for the case of bulk emission in this study, we have nearest neighbors along the [001] azimuth with $\mathrm{R}_{\mathrm{nn}}=$ $2.74 \AA$ at $\theta_{k}=53^{\circ}$, and next-nearest neighbors along the [[1 $\left.\overline{1} 0\right]$ azimuth with $R_{\mathrm{nn}}=$ $3.16 \AA$ at $\theta_{\mathrm{k}}=45^{\circ}$, leading to peak rotations of $16.2^{\circ}$ and $17.9^{\circ}$ respectively. According to this model, Fig. 6.12 shows where these forward scattering peaks should be rotated to, and there is reasonable agreement with experiment for some features but not others. For example, the pair of peaks centered on $\phi=90^{\circ}$ and $270^{\circ}$ in the LP data appear to behave approximately as predicted by Eq. 6.10 in the RCP and LCP data, as do weaker shoulders 
on the strong peak centered at $\phi=180^{\circ}$. However, the strongest peaks at $\phi=0^{\circ}, 90^{\circ}$, and $180^{\circ}$ do not show any evidence of such rotations. Further discussion of applying this model to the O/W system appears elsewhere. [10].

A more accurate description of these effects is to include all allowed transitions with their precise weightings, as well as single and multiple scattering of the outgoing photoelectron wave. Such calculations have been applied successfully to $\operatorname{Si}(001)$ by Kaduwela et al. [5(b)], and we here apply them to our data for $\mathrm{O} / \mathrm{W}$. These calculations were performed using a program newly developed by García [9(c)] which includes a full treatment of the multiple scattering, and were checked against the program developed by Kaduwela et al. [8(a)] which is in turn based on the Rehr-Albers approximation for treating multiple scattering [8(b)]. The non-structural parameters used are the same as those used in the structure determination for $\mathrm{O} / \mathrm{W}(110)(1 \times 1) \times 12$, and they are found elsewhere [7(c),8].

The resulting normalized theoretical intensities and $\mathrm{CDAD}$ values for oxide and bulk emission are compared directly to experiment as the grey curves in Figs. 6.2-6.7 and experiment and theory for the full data set are also compared in Figs. 6.10 and 6.11. In general, diffraction theory at this level correctly predicts not only the gross motion of certain features that one can often describe as rotations, but also various other peak distortions and fine structure beyond this simple description. Considering first intensities in more detail, the more stringent curve-to-curve comparisons of Figs. 6.2-6.7 show some discrepancies between experiment and theory, especially for oxide emission with linear 
polarization, but the global grey-scale comparisons over the full data sets in Figs. 6.10 and 6.11 are in general in excellent agreement as to all major changes with polarization, whether they can be interpreted as rotations or more complex alterations in fine structure. The bulk intensities in Figs. 6.5-6.7 are predicted somewhat more accurately for all three polarizations than the oxide intensities in Figs. 6.2-6.4, with theory generally showing more fine structure than experiment, perhaps due to an effective degree of experimental angular averaging that is larger than that included in theory. The CDAD comparisons for oxide in the bottom panels of Figs. 6.2-6.4 also show excellent agreement between experiment and theory, even as to certain narrower features, as for example in Fig. 6.2 around $\phi=180^{\circ}$. The fact the agreement for CDAD behavior is actually better than for the normalized oxide intensities suggests that predicting such $\mathrm{CDAD}$ effects may be easier than actually calculating the two separate RCP and LCP intensities involved. The magnitude of the theoretical CDAD effects is in Figs. 6.2 and 6.3 about 1.5-2.0 times larger than experiment, but this could be due to inadequate angular averaging or other simplifications we have made in dealing for example with inelastic or vibrational effects in theory. The CDAD comparisons for bulk in the bottom panels of Figs. 6.5-6.7 also show reasonable agreement between experiment and theory, with theory again predicting larger effects, this time by about 2-3 times. For this multilayer emission case that is expected to involve more multiple scattering, it is interesting that the intensities appear to be predicted somewhat more accurately than the $\mathrm{CDAD}$ values, or the converse of the situation for the oxide data. Thus, we conclude that a quantitative description of nonmagnetic $\mathrm{CDAD}$ is possible within this multiple scattering model, but that further 
refinements of the theory may be necessary to provide agreement at the few $\%$ level that may be necessary to quantitatively describe small magnetic circular dichroism effects [3].

The azimuthal variation of the CDAD effects can also be better understood if we look back at Eq. 6.5. Here, we see that the intensities for circularly polarized light should both exhibit the same two leading terms $\left(I_{L P x}+I_{L P y}\right)$ and that these should show a basic pattern that is symmetric about the low index directions, since $\mathrm{I}_{\mathrm{LPx}}$ and $\mathrm{I}_{\mathrm{LPy}}$ have the same symmetry as the sample in our experimental geometry. Added onto this is a term proportional to $\sin \Delta$, where $\Delta$ is the phase between the two integrals $\left\langle\phi_{\mathrm{f}}|\mathrm{x}| \phi_{n} l_{m}\right\rangle$ and $\left\langle\phi_{f}|y| \phi_{n l m}\right\rangle^{*}$, which alters the intensity away from low-index directions that in turn contain mirror planes perpendicular to the surface (such as $\phi=0^{\circ}, 90^{\circ}, 180^{\circ}, 270^{\circ}$ in the present case). This term will thus control the angular dependence of the CDAD, which must have mirror symmetry around such low-index directions, as seen in both experiment and theory in Figs. 6.2-6.7.

Overall, we conclude that, although the model of Daimon et al. [4(a)] correctly describes the basic physics of the peak rotation phenomenon, a fully quantitative description of such non-magnetic $\mathrm{CDAD}$ effects will require a complete treatment of the excitation matrix elements, including photoelectron scattering and diffraction.

Finally we note the interesting result that, in our data, the sum of $I_{L C P}$ and $I_{R C P}$ is found to be very nearly equal to $\mathrm{I}_{\mathrm{LPx}}$, as illustrated in Figs. 6.13(a)-(c) for the oxide data and in Figs. 6.14(a)-(c) for the bulk data at the same three takeoff angles. The 
corresponding theoretical curves are shown in Figs. 6.13(d)-(f) and 6.14(d)-(f), and they verify that this is indeed an expected result, with the actual form of the sum $I_{L C P}+I_{R C P}$ for bulk intensities again being predicted more accurately than those of oxide, as seen already for the individual intensities in Figs. 6.2-6.7. Stereographic projections of the full experimental data sets are shown in Fig. 6.15, including the sum $I_{L C P}+I_{R C P}$ and $I_{L P x}$ for both bulk and oxide. Again, the near identity is striking. This result is not rigorously true for all cases, but can be shown to be a good approximation for $\mathrm{W} 4 \mathrm{f}$ in our geometry and for our kinetic energy range. That is, from Eq. 6.4, in order for our observed relationship $I_{L P x} \propto I_{L C P}+I_{R C P}$ to hold, the contribution of $I_{L P y}$ must either be much less than that of $\mathrm{I}_{\mathrm{LPx}}$, or very nearly equal to it. Neglecting diffraction effects, the relative intensities of $\mathrm{I}_{\mathrm{LPx}}$ and $\mathrm{I}_{\mathrm{LPy}}$ can be calculated from the atomic W4f differential photoelectric crosssections $\mathrm{d} \sigma / \mathrm{d} \Omega$ for the two polarizations, which depend in turn on the total subshell cross section $\sigma$ and the asymmetry parameter $\beta$ :

$\frac{\mathrm{d} \sigma(\gamma)}{\mathrm{d} \Omega}=\frac{\sigma}{4 \pi}\left[1+\beta\left(3 / 2 \cos ^{2} \gamma-1 / 2\right)\right]$

where the angle $\gamma$ is between the polarization vector and the wave vector $\mathbf{k}$, and is thus equal to $\theta$ for $\mathrm{x}$ polarization and $90^{\circ}$ for y polarization in our experimental geometry (cf. Fig. 6.1(a)). The quantities $\sigma$ and $\beta$ have been determined from tabulations that appear in the literature [11]. The angular dependent term in the differential cross section is multiplied by the $\beta$ parameter, and thus the angular dependence is smaller when $\beta$ is smaller, with $d \sigma / d \Omega$ isotropic when $\beta=0$. Figures 6.16(a) and (b) show both $\sigma$ and $\beta$ as 
a function of photon energy over a broad range from $40 \mathrm{eV}$ to $1486 \mathrm{eV}$ (a typical XPS energy). We note that $\beta$ has its minimum at $160 \mathrm{eV}$ and is only about 0.45 at our photon energy of $280 \mathrm{eV}$. With our experimental geometry and electron kinetic energy of 250 $\mathrm{eV}$, the differential cross section is thus rather isotropic compared to that at higher XPS kinetic energies, with the actual forms being plotted in Figs. 6.16 (c),(d). In Fig. 6.16(d), we have indicated the value or range of $\gamma$ that is important for each polarization, and these are within a factor of 1.4-1.6 of one another over the entire range studied. This near equality causes the $\mathrm{x}$ and $\mathrm{y}$ polarizations to behave very similarly and qualitatively explains our observation that $\mathrm{I}_{\mathrm{LPx}} \propto \mathrm{I}_{\mathrm{LCP}}+\mathrm{I}_{\mathrm{RCP}}$. As a final comment on this near-equality, we note that it offers a complementary interpretation to peak rotation for qualitatively understanding the changes in diffraction patterns which occur on switching from RCP to LCP. That is, with LP, features due to both RCP and LCP are present, and by choosing either one of the two, we simply eliminate the other. Thus, using circular polarization results in a reduction in the number of diffraction features, with some of the differences between RCP and LCP being describable as peak rotations.

\section{V. CONCLUSIONS}

Circular dichroism in core photoelectron angular distributions for non-magnetic systems can result in large differences in $\mathrm{I}_{\mathrm{LCP}}$ and $\mathrm{I}_{\mathrm{RCP}}$ of up to $\pm 50 \%$, and such effects are expected to be very generally observable. Our results for a saturated monolayer coverage of $\mathrm{O}$ on $\mathrm{W}(110)$ are qualitatively similar to prior work on $\mathrm{Si}(001)$ by Daimon et al. [4], but they further show that these effects can arise for different chemical states in a 
single sample, and that they can be predicted quantitatively by photoelectron diffraction calculations including multiple scattering effects. These calculations provide a more quantitative approach to analyzing such data than a model previously-proposed by Daimon et al. and based upon considerations of peak rotations of forward scattering effects in photoelectron diffraction. We also note that, depending on the energy and geometry in which such measurements are performed, the sum of intensities with right and left polarization may be nearly equal to that of a single linear polarization, a potentially useful result for checking experimental self-consistency, especially since both energy and geometry can be varied in many present experimental systems. E.g., tuning the energy such that the parameter $\beta$ is either near zero or near 2.0 would in a geometry such as ours lead to a near-equality of the sum of left and right intensities and the linear intensity. Such non-magnetic circular dichroism effects due to photoelectron diffraction must also be included in any interpretation of analogous $\mathrm{MCD}$ experiments with magnetic materials. For example, in photoemission from the $2 \mathrm{p}$ levels of the $3 \mathrm{~d}$ ferromagnetic metals, MCD is only a few \% in magnitude [3(a)], and thus potentially much smaller than the non-magnetic contributions [4,5]. By contrast, for the high-spin $4 f$ ferromagnet $\mathrm{Gd}$, MCD has been found to be about $\pm 35 \%$, and thus about 7 times larger than the nonmagnetic diffraction contributions at $\pm 5 \%[3(\mathrm{~d}), 3(\mathrm{e})]$; nonetheless, even for this case the non-magnetic diffraction effects cannot be fully neglected. 


\section{References:}

[1] G. Schutz, W. Wagner, W. Wilhelm, P. Kienle, R. Frahm, and G. Materlik, Phys. Rev. Lett. 58, 737 (1987); G. Schutz, M. Knulle, R. Wienke, W. Wilhelm, W. Wagner, P. Kienle, and R. Frahm, Z. Phys. B 73, 67 (1988); G. Schutz, R. Frahm, P. Mautner, R. Wienke, W. Wagner, W. Wilhelm, and P. Kienle, Phys. Rev. Lett. 62, 2620 (1989); C.T. Chen, F. Sette, Y. Ma, and S. Modesti, Phys. Rev. B 42, 7262 (1990); C.T. Chen, N.V. Smith, and F. Sette, Phys. Rev. B 43, 6785 (1991); L.H. Tjeng, Y.U. Idzerda, P. Rudolf, F. Sette, and C.T. Chen, J. Magn. Magn. Mat. 109, 288 (1992); J. Stöhr, Y. Wu, B.D. Hermsmeier, M.G. Samant, G.R. Harp, S. Koranda, D. Dunham, and B.P. Tonner, Science 259, 658 (1993).

[2] (a) G. Schönhense, Physica Scripta T 31, 255 (1990); (b) C. Westphal, J. Bansmann, M. Getzlaff, and G. Schönhense, Phys. Rev. Lett. 63, 151 (1989); (c) J. Bansmann, Ch. Ostertag, G. Schönhense, F. Fegel, C. Westphal, M. Getzlaff, F. Schafers, and H. Peterson, Phys. Rev. B 46, 13496 (1992); (d) C. Westphal, A.P. Kaduwela, C.S. Fadley, and M.A. Van Hove, Phys. Rev. B 50, 6203 (1994).

[3] (a) L. Baumgarten, C.M. Schneider, H. Peterson, F. Schafers, and J. Kirschner, Phys. Rev. Lett. 65, 492 (1990); (b) J.G. Tobin, G.D. Waddill, and Pappas, Phys Rev. Lett. 68, 3642 (1992); (c) G.D. Waddill, J.G. Tobin, and Pappas, Phys Rev. B 46, 552 (1992); (d) C.S. Fadley et al., Prog. in Surf. Sci. 54, 341 (1997); (e) R. Denecke, J. Morais, et al., unpublished results for $\mathrm{Gd}$.

[4] (a) H. Daimon, T. Nakatani, S. Imada, S. Suga, Y. Kagoshima, and T. Miyahara, Jpn. J. Appl. Phys. 32, L1480 (1993); (b) H. Daimon, T. Nakatani, S. Imada, and S. Suga, J. Elec. Spec. Rel. Phen. 76, 55 (1995). 
[5] (a) C. Westphal, A.P. Kaduwela, C.S. Fadley, and M.A. Van Hove, Phys. Rev. B, Rapid Comm. 50, 9656 (1994); (b) A.P. Kaduwela, H. Xiao, S. Thevuthasan, C.S. Fadley, and M.A. Van Hove, Phys. Rev. B 52, 14927 (1995).

[6] (a) Z. Hussain, W. R. A. Huff, S. A. Keller, E. J. Moler, P. A. Heimann, W. McKinney, H. A. Padmore, C. S. Fadley, and D. A. Shirley, J. Elec. Spec. and Rel. Phen. 80, 401 (1996); (b) J.B. Kortright, M.Rice, Z. Hussain, H.A. Padmore, A. Adamson, W.R.A. Huff, A.T. Young, E.J. Moler, S.A. Kellar, R.X. Ynzunza, F.J. Palormares, H.Daimon, E.D. Tober, C.S. Fadley, Rev. Sci. Instrum. 67(9) (1996).

[7] (a) K.E. Johnson, R.J. Wilson, and S. Chiang, Phys. Rev. Lett. 71, 1055 (1993);

(b) H. Daimon, R.X. Ynzunza, J. Palomares, H. Takagi, and C.S. Fadley, Surf. Sci. 408, 260 (1998).; (c) R. Ynzunza et al., to be published.

[8] R.X. Ynzunza, Ph.D. thesis (University of California-Davis, 1998), Chapters 2 and 4.

[9] (a) A.P. Kaduwela, D.J. Friedman, and C.S. Fadley, J. Elec. Spectrosc. and Relat. Phenom. 57, 223 (1991); (b) J.J Rehr and R.C. Albers, Phys. Rev. B 41, 81139 (1990); (c) F.J. García de Abajo et al., Phys. Rev. B, to appear.

[10] H.Daimon, R.X. Ynzunza, F.J. Palomares, E.D. Tober, Z.X. Wang, A.P. Kaduwela, M.A. Van Hove, and C.S. Fadley, Phys. Rev. B, to appear.

[11] S.M. Goldberg, C.S. Fadley, and S. Kono, J. Electron. Spectrosc. 21, 285 (1981); J.J. Yeh and I. Lindau, Atomic Data and Nuclear Data Tables 32, 1 (1985). 
a)

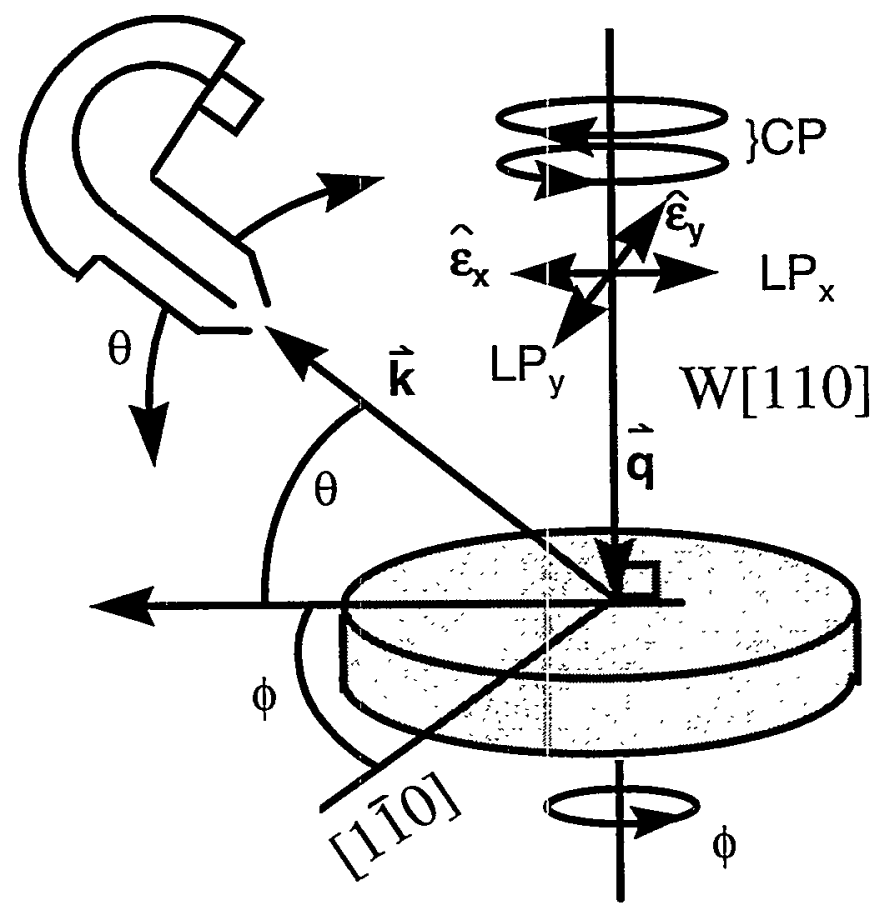

b)

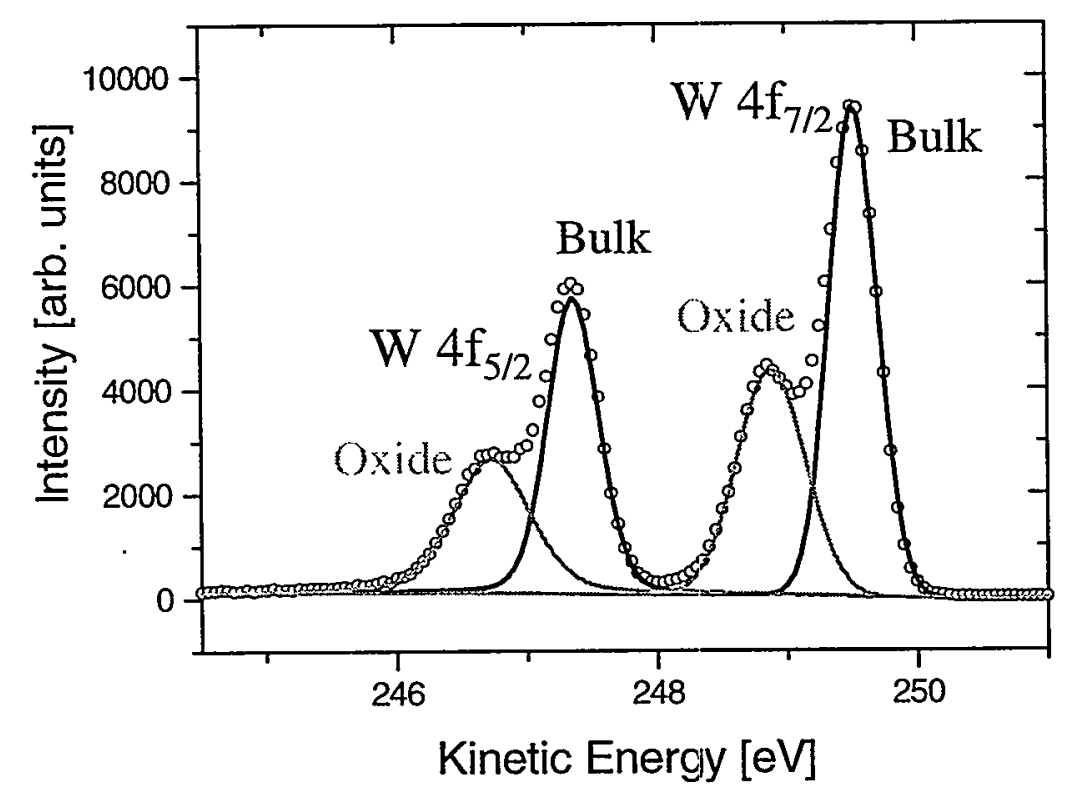

Figure 6.1. a) The experimental geometry, with the polarization of the incoming light kept perpendicular to the surface normal. Variation of electron polar (takeoff) angle $\theta$ is achieved by rotating the Scienta photoelectron analyzer; variation of electron azimuthal angle $\phi$ by rotating the sample. The orientation of the $\mathrm{x}$ linear polarization $\left(\widehat{\varepsilon}_{x}\right)$ is also indicated; $y$ linear polarization $\left(\widehat{\varepsilon}_{y}\right)$ is perpendicular to the plane containing $\overrightarrow{\mathrm{q}}$ and $\overrightarrow{\mathrm{k}}$, and is shown only for reference in discussing our results. b) A typical $\mathrm{W}$ 4f spectrum along the $<001>$ azimuthal direction $\left(\phi=90^{\circ}\right)$ and at a $44^{\circ}$ polar angle, with oxide and bulk peaks resolved in both the $7 / 2$ and $5 / 2$ components. 


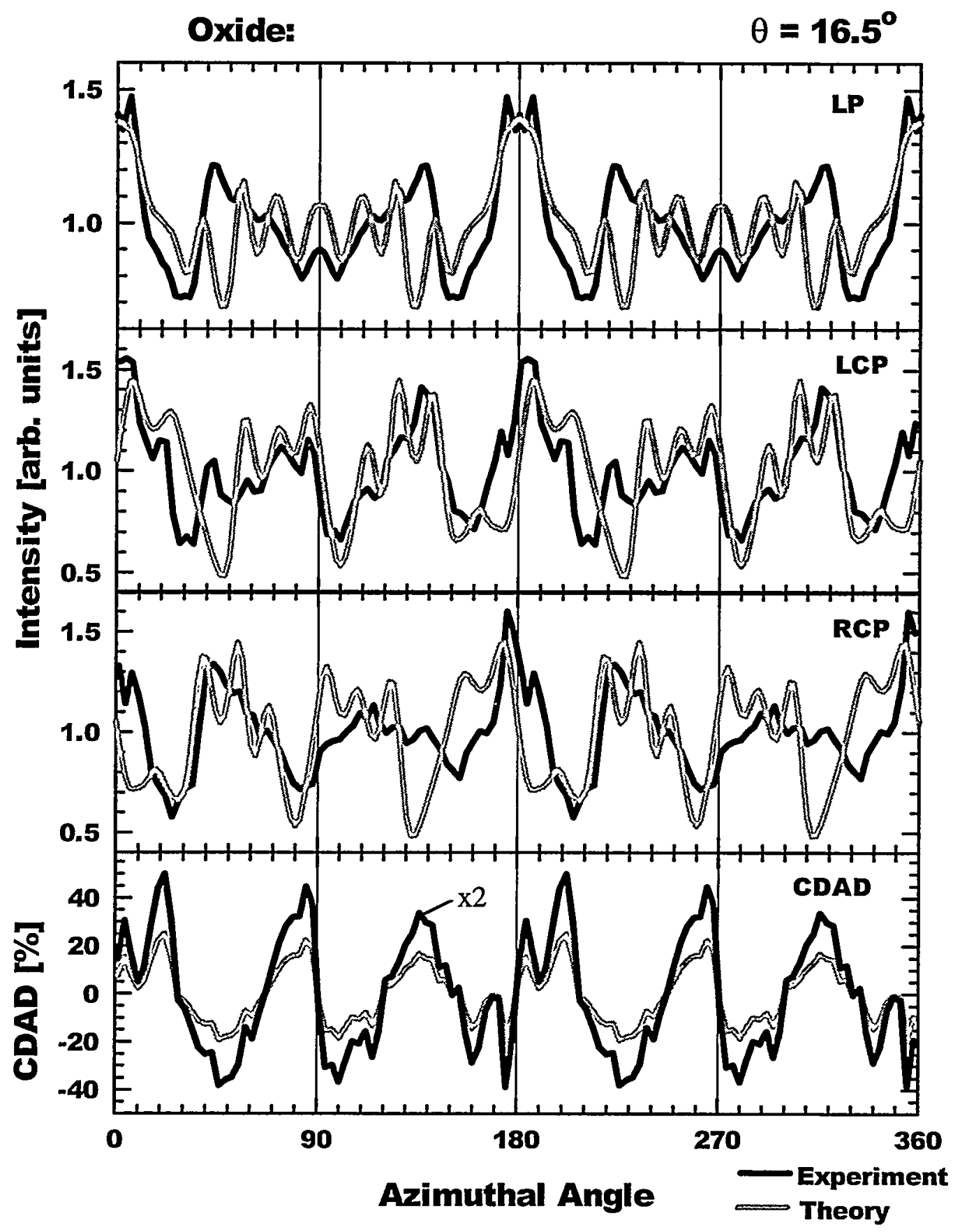

Figure 6.2. Experimental (black) and theoretical (grey) azimuthal scans of W4f oxide intensity at a takeoff angle of $16.5^{\circ}$ for linearly polarized light (LP), left circularly polarized light (LCP), and right circularly polarized light (RCP), together with the circular dichroism in the angular distribution (CDAD), as defined in the text. The experimental CDAD values have here been multiplied by 2 . 


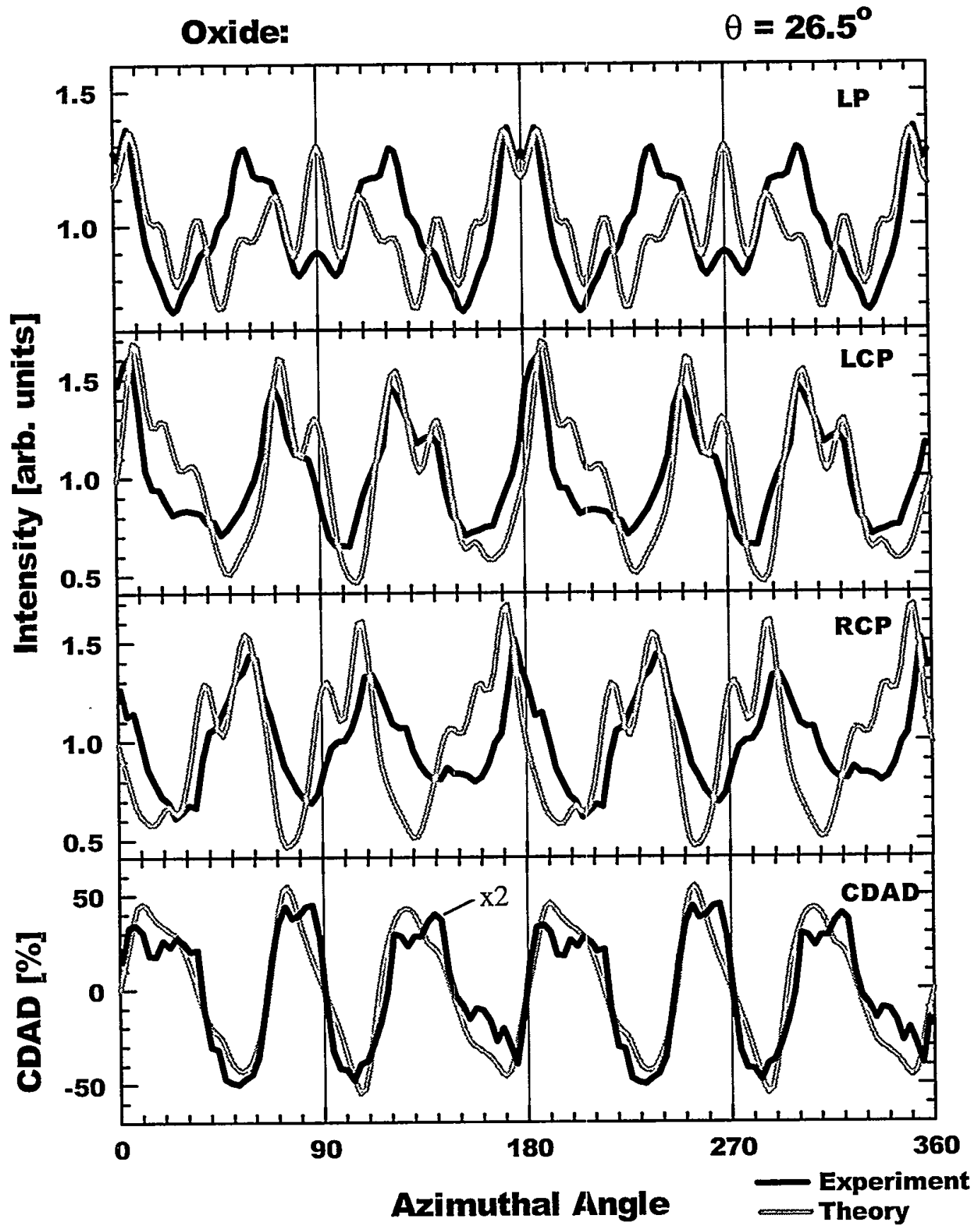

Figure 6.3. Experimental (black) and theoretical (grey) azimuthal scans of W4f oxide intensity at a takeoff angle of $26.5^{\circ}$ for linearly polarized light (LP), left circularly polarized light (LCP), and right circularly polarized light (RCP), together with the circular dichroism in the angular distribution (CDAD), as defined in the text. The experimental CDAD values have here been multiplied by 2 . 


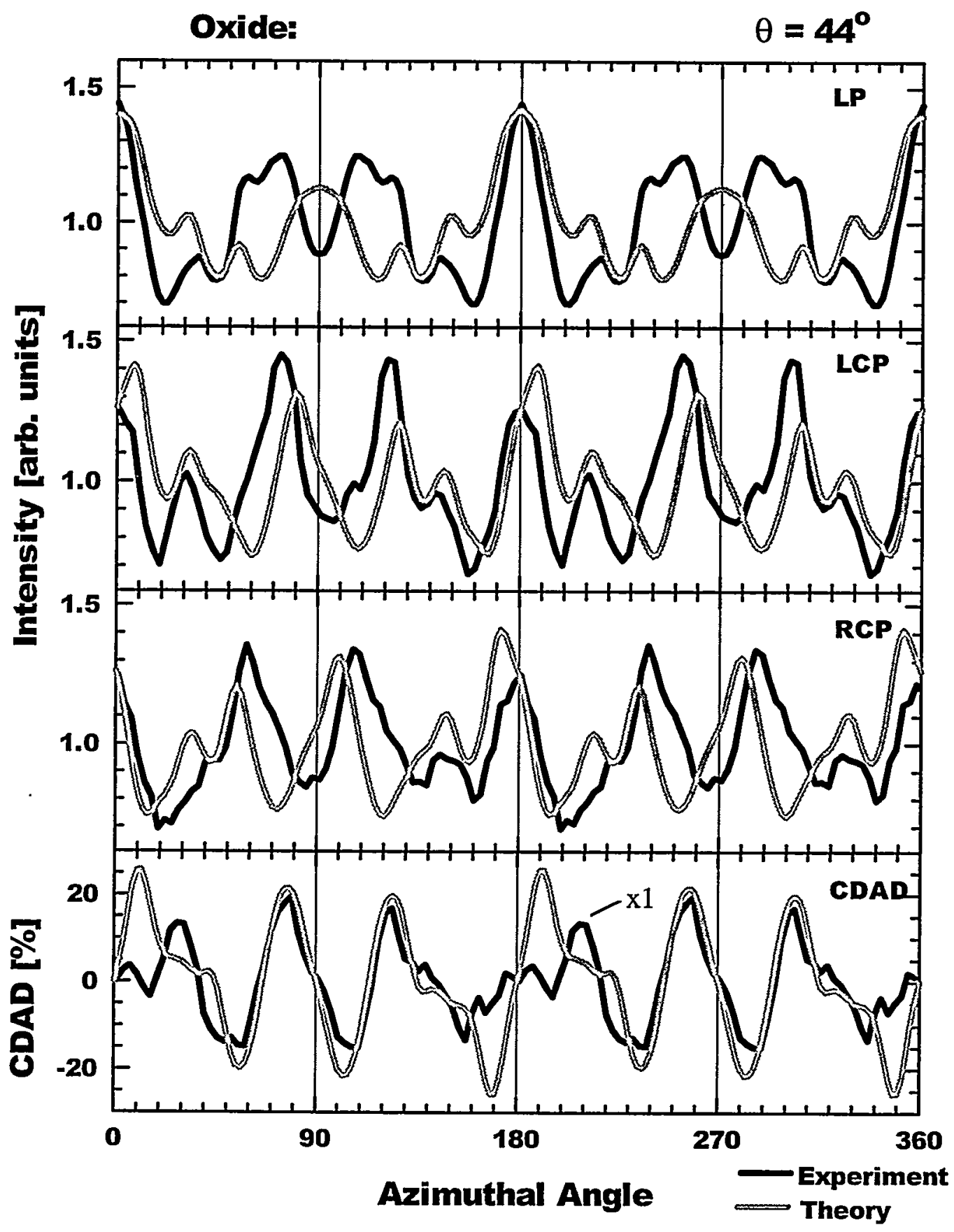

Figure 6.4. Experimental (black) and theoretical (grey) azimuthal scans of W4f oxide intensity at a takeoff angle of $44^{\circ}$ for linearly polarized light (LP), left circularly polarized light (LCP), and right circularly polarized light (RCP), together with the circular dichroism in the angular distribution (CDAD), as defined in the text. 


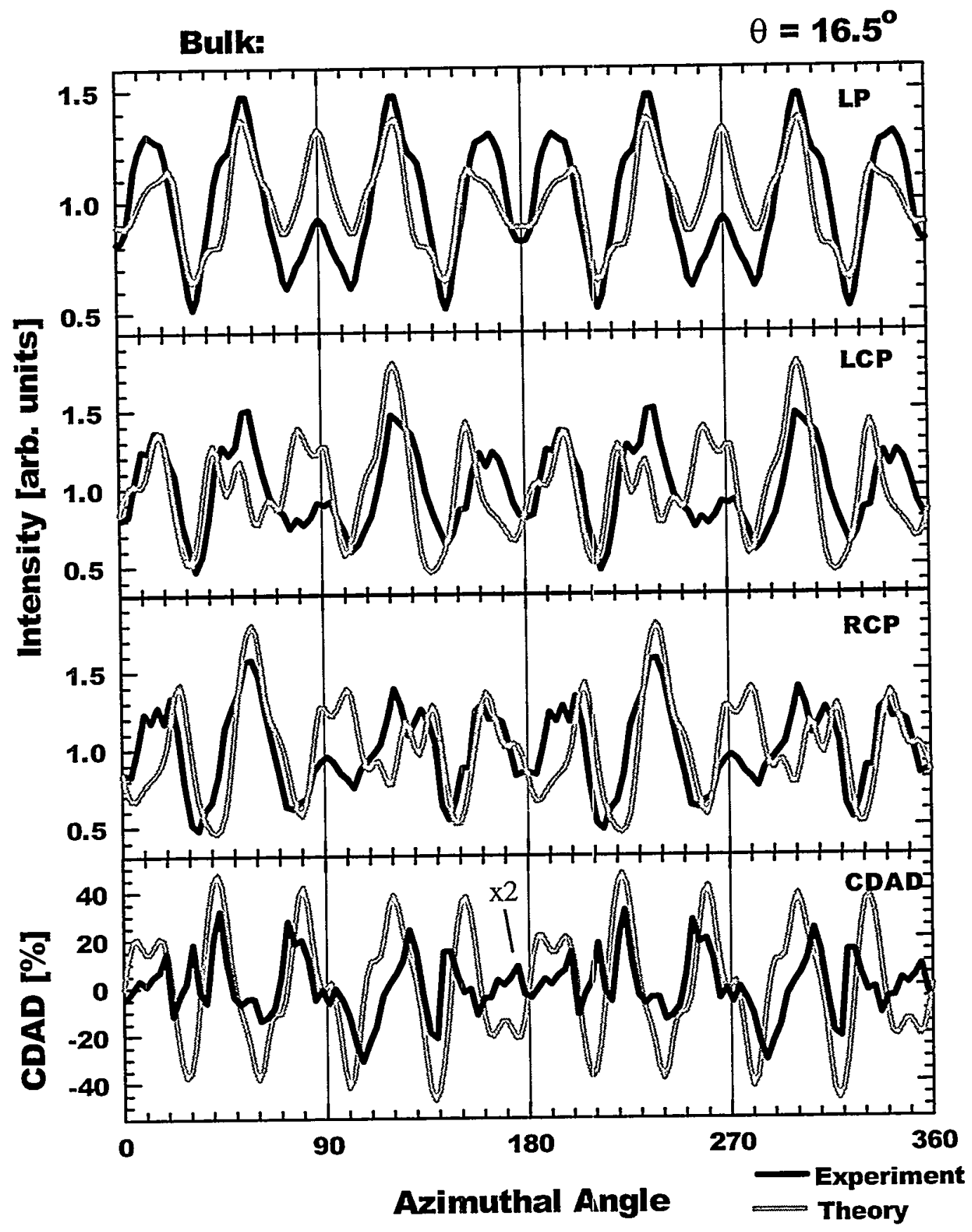

Figure 6.5. Experimental (black) and theoretical (grey) azimuthal scans of W4f bulk intensity at a takeoff angle of $16.5^{\circ}$ for linearly polarized light (LP), left circularly polarized light (LCP), and right circularly polarized light (RCP), together with the circular dichroism in the angular distribution (CDAD), as defined in the text. The experimental CDAD values have here been multiplied by 2 . 


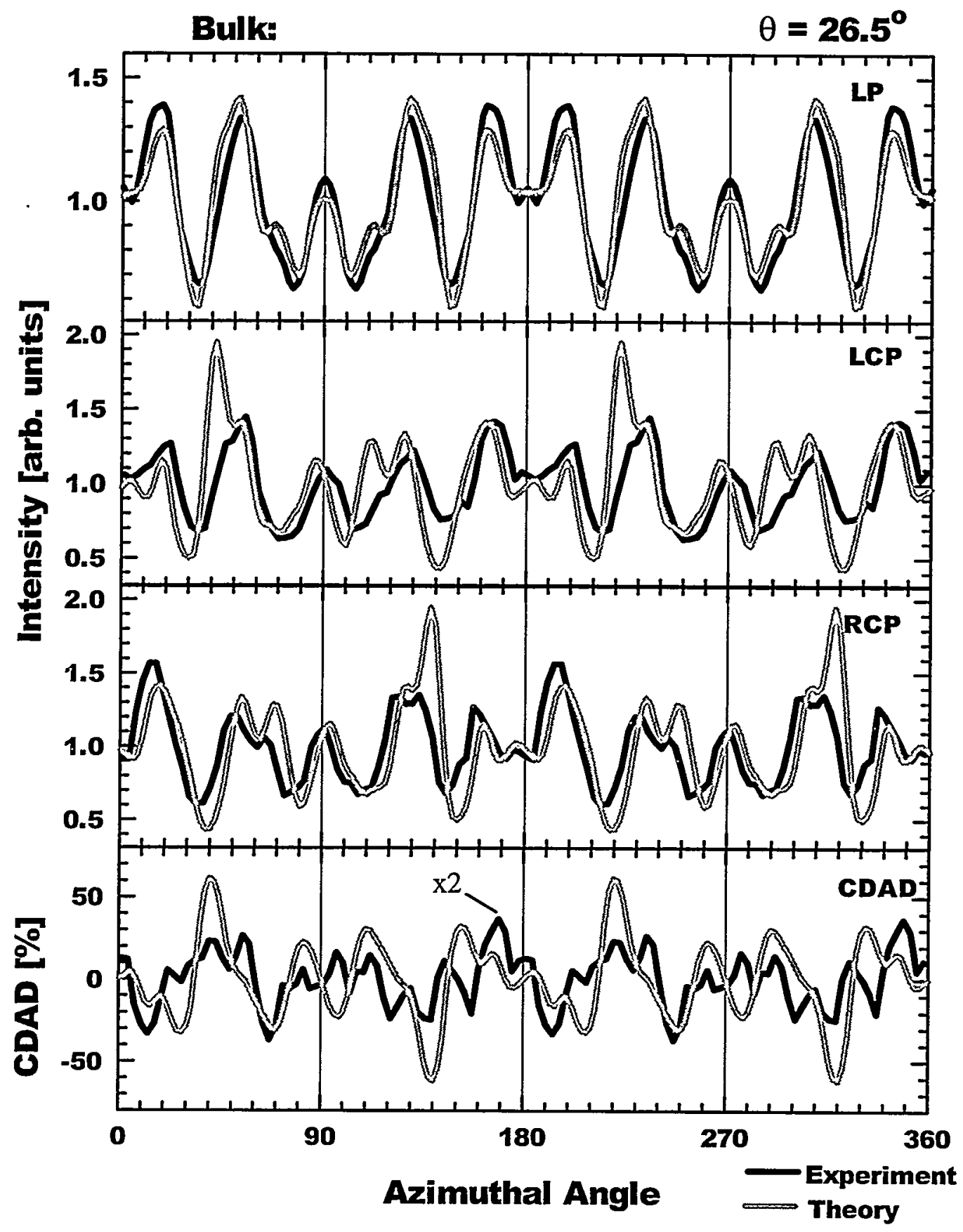

Figure 6.6. Experimental (black) and theoretical (grey) azimuthal scans of W4f bulk intensity at a takeoff angle of $26.5^{\circ}$ for linearly polarized light (LP), left circularly polarized light (LCP), and right circularly polarized light (RCP), together with the circular dichroism in the angular distribution (CDAD), as defined in the text. The experimental CDAD values have here been multiplied by 2 . 


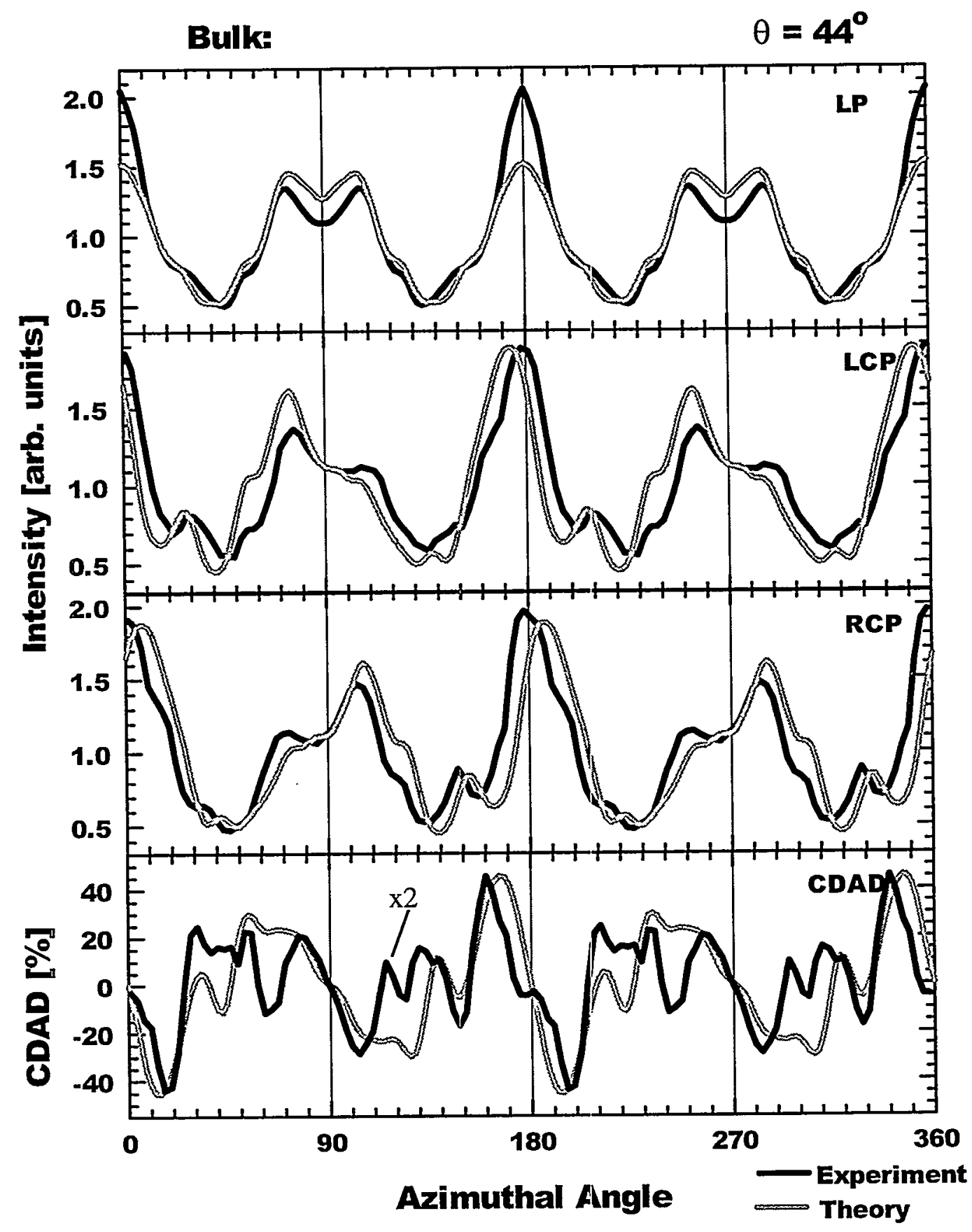

Figure 6.7. Experimental (black) and theoretical (grey) azimuthal scans of W4f bulk intensity at a takeoff angle of $44^{\circ}$ for linearly polarized light (LP), left circularly polarized light (LCP), and right circularly polarized light (RCP), together with the circular dichroism in the angular distribution (CDAD), as defined in the text. The experimental CDAD values have here been multiplied by 2 . 


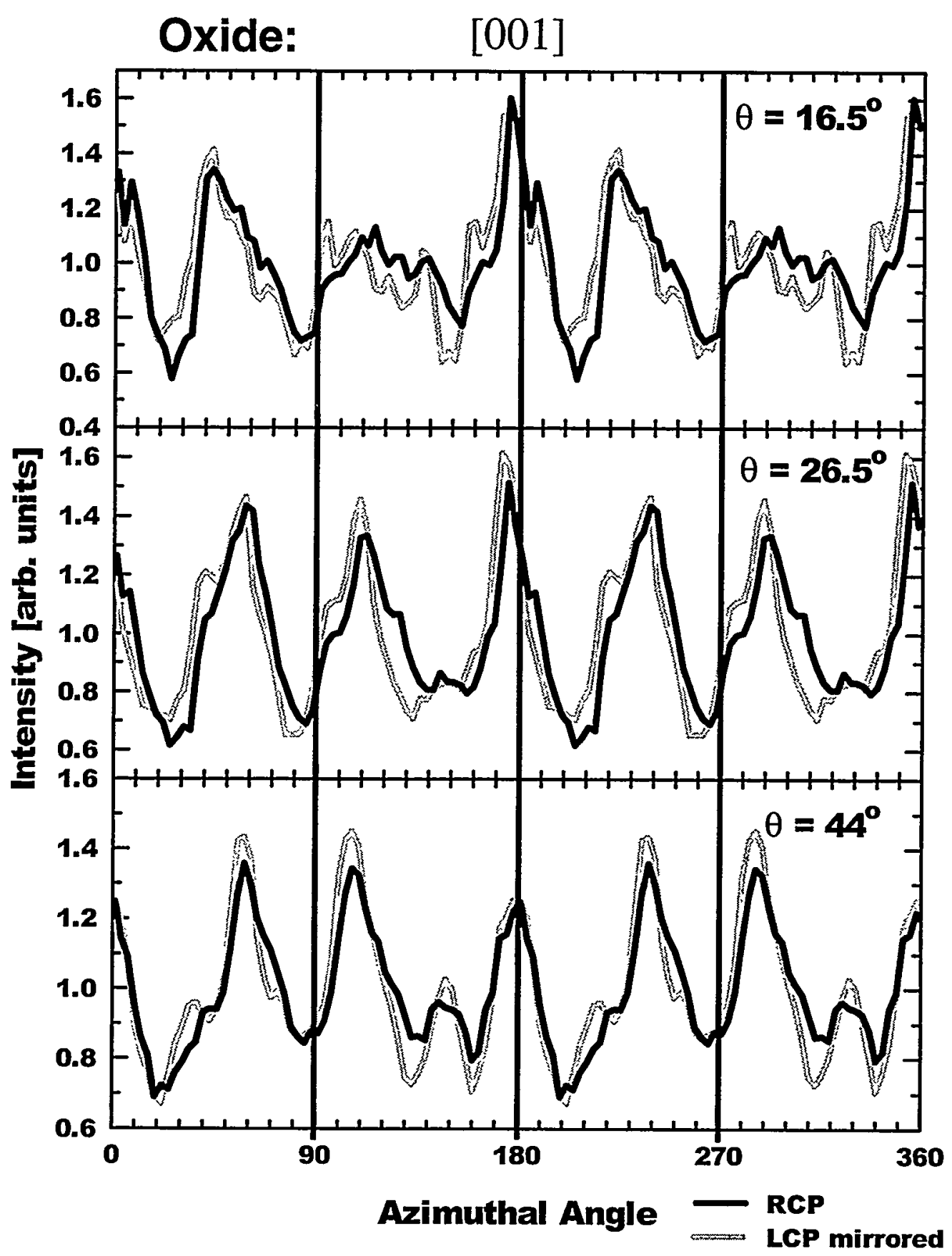

Figure 6.8. Experimental azimuthal scans of $\mathrm{W} 4 \mathrm{f}$ oxide intensity. The black curves are the original RCP data at the takeoff angles of $\theta=16.5^{\circ}, 26.5^{\circ}$, and $44^{\circ}$. The grey curves are the corresponding LCP data after a mirror reflection across the [001] azimuthal direction. 


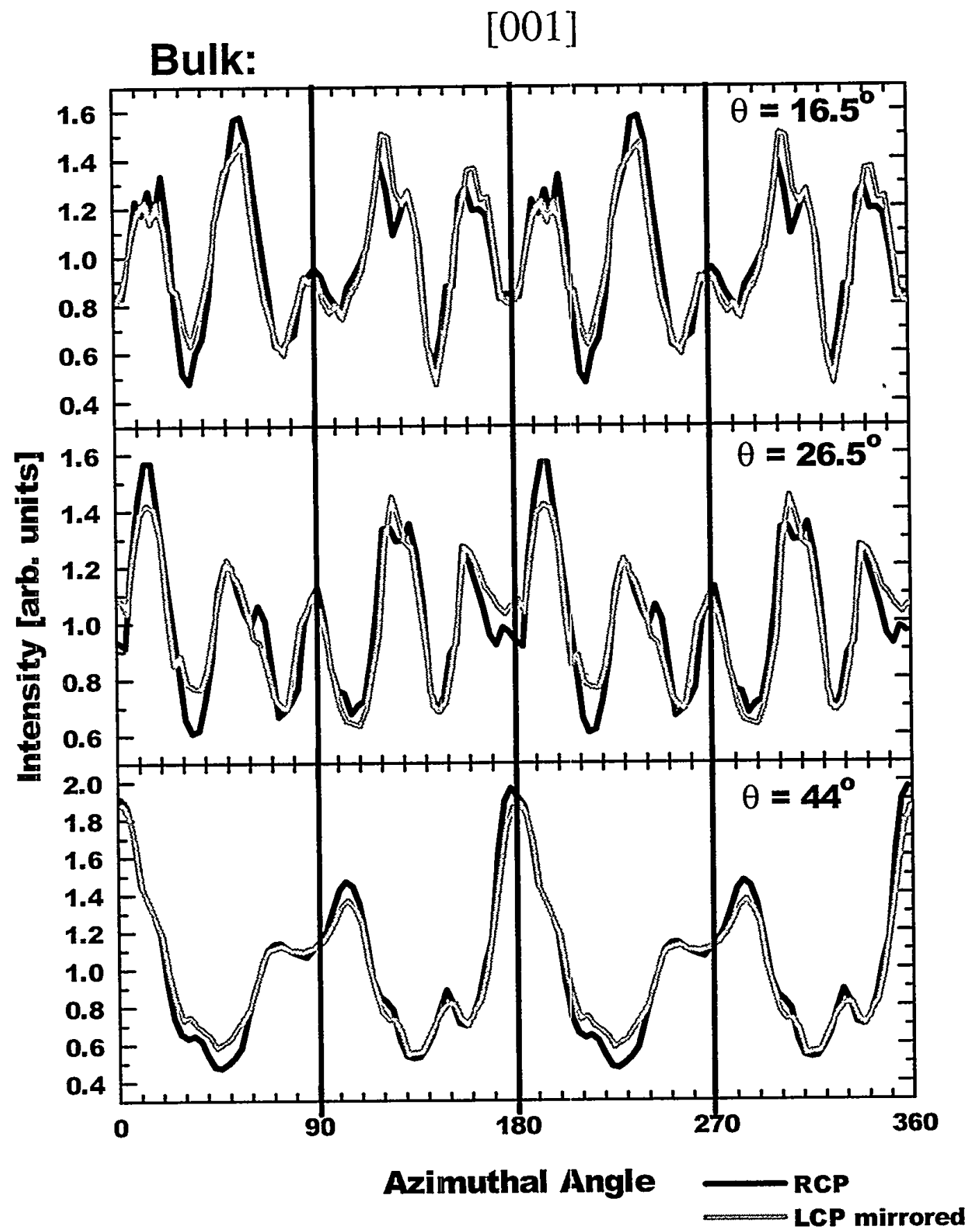

Figure 6.9. Experimental azimuthal scans of $\mathrm{W} 4 \mathrm{f}$ bulk intensity. The black curves are the original RCP data at the takeoff angles of $\theta=16.5^{\circ}, 26.5^{\circ}$, and $44^{\circ}$. The grey curves are the corresponding LCP data after a mirror reflection across the [001] azimuthal direction. 


\section{Experiment}

a)

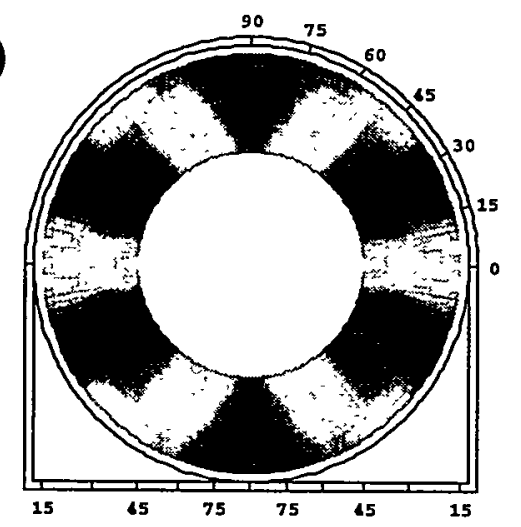

$\underline{\mathrm{LP}}$

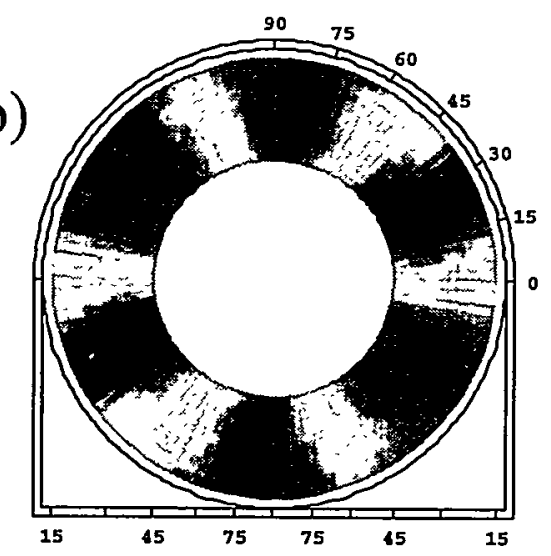

c)

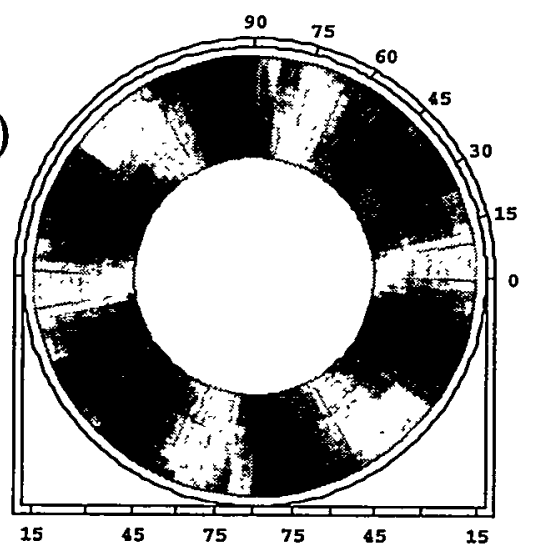

\section{$\underline{\text { Oxide }}$}

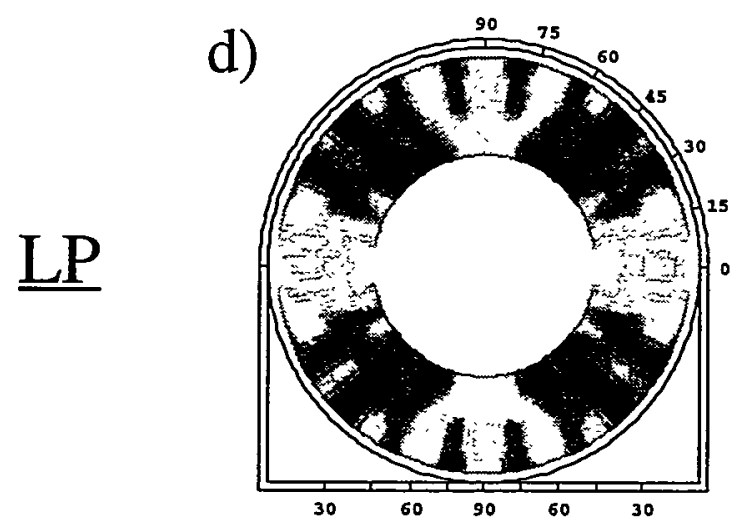

$\underline{\mathrm{RCP}}$

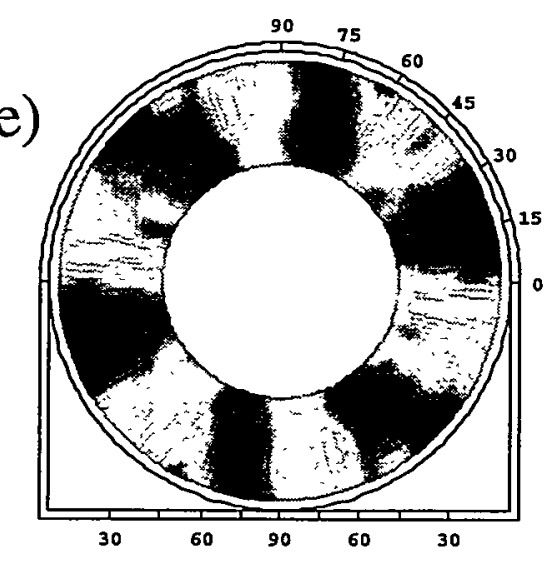

$\underline{\mathrm{LCP}}$

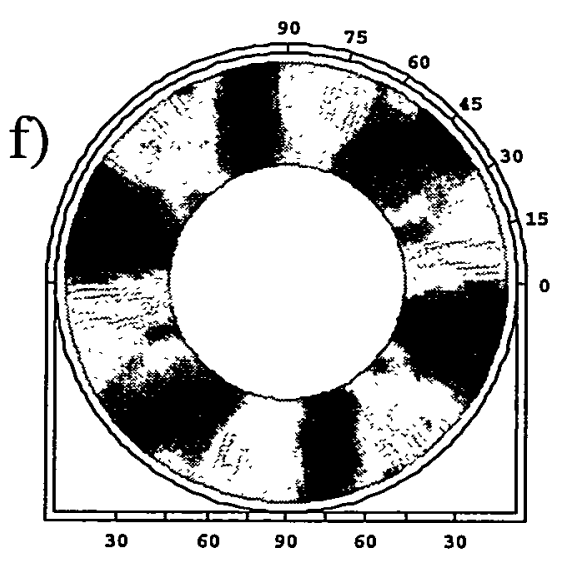

Figure 6.10. Large-solid-angle photoelectron diffraction patterns for the normalized W4f oxide intensity: Experimental -- a) linearly polarized light, b) RCP light, and c) LCP light; Theoretical -- d) linearly polarized light, e) RCP light, and f) LCP light. 


\section{$\underline{\text { Bulk }}$}

\section{Experiment}

a)

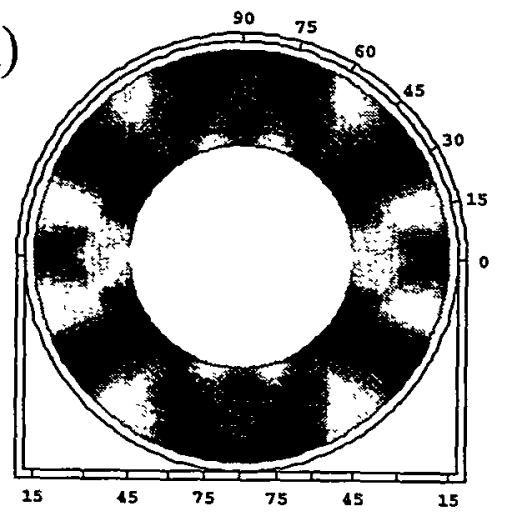

b)

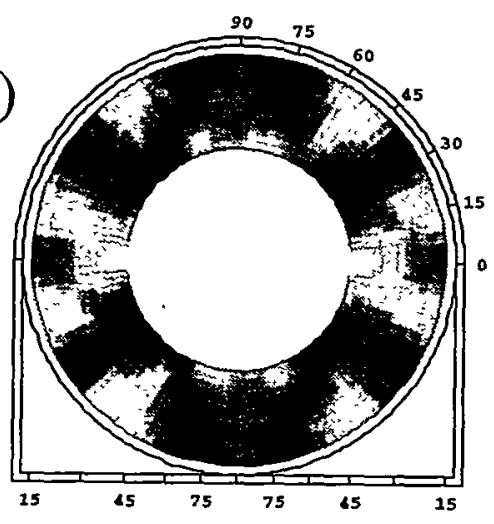

c)

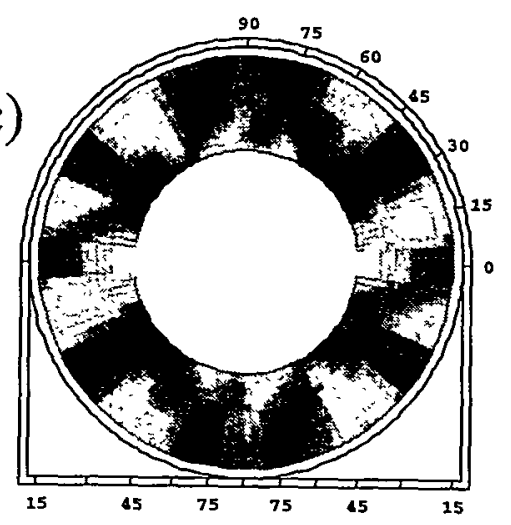

$\underline{\mathrm{LP}}$
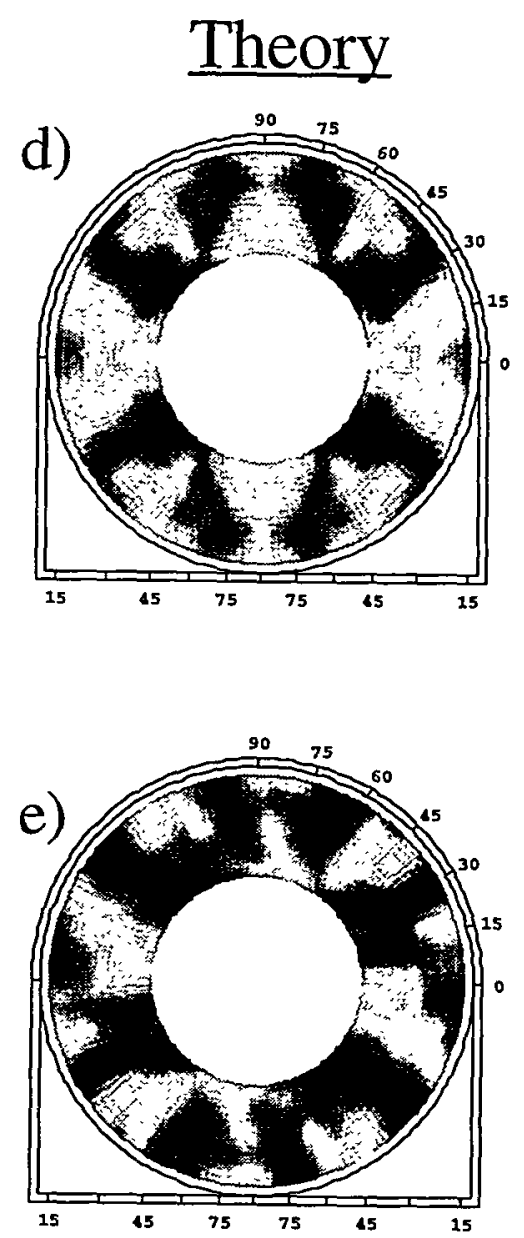

$\underline{\mathrm{LCP}}$

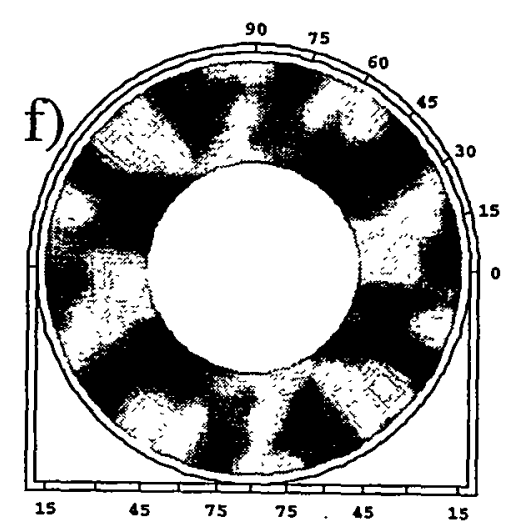

Figure 6.11. Large-solid-angle photoelectron diffraction patterns for the normalized W4f bulk intensity: Experimental -- a) linearly polarized light, b) RCP light, and c) LCP light; Theoretical -- d) linearly polarized light, e) RCP light, and f) LCP light. 


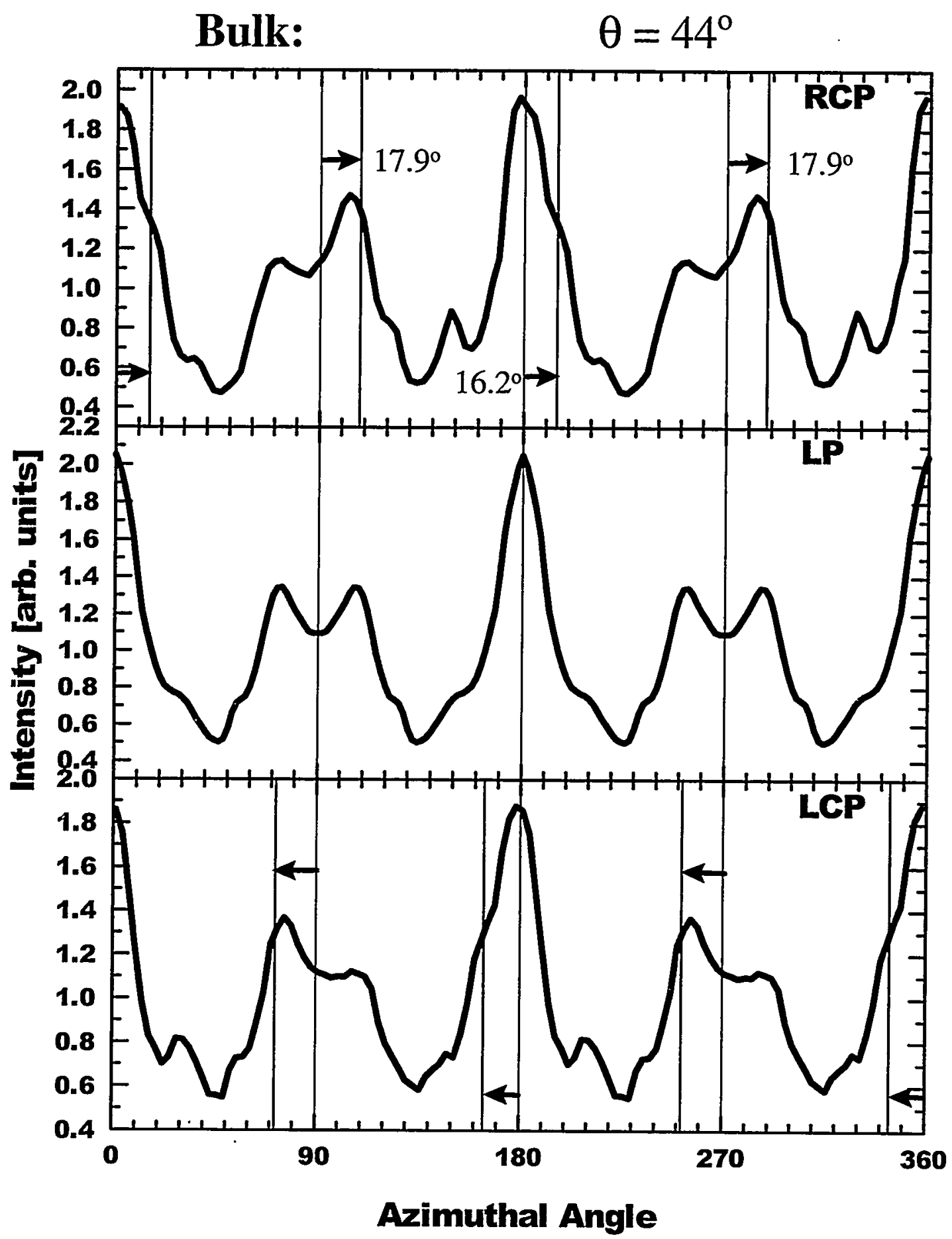

Figure 6.12. Experimental azimuthal diffraction patterns for the W4f bulk intensity at $\theta=44^{\circ}$ and for the three polarizations: RCP, LP, and LCP. Vertical lines correspond to expected peak rotations of $16.2^{\circ}$ and $17.9^{\circ}$ from forward scattering directions, as calculated from Eq. 6.10 . 


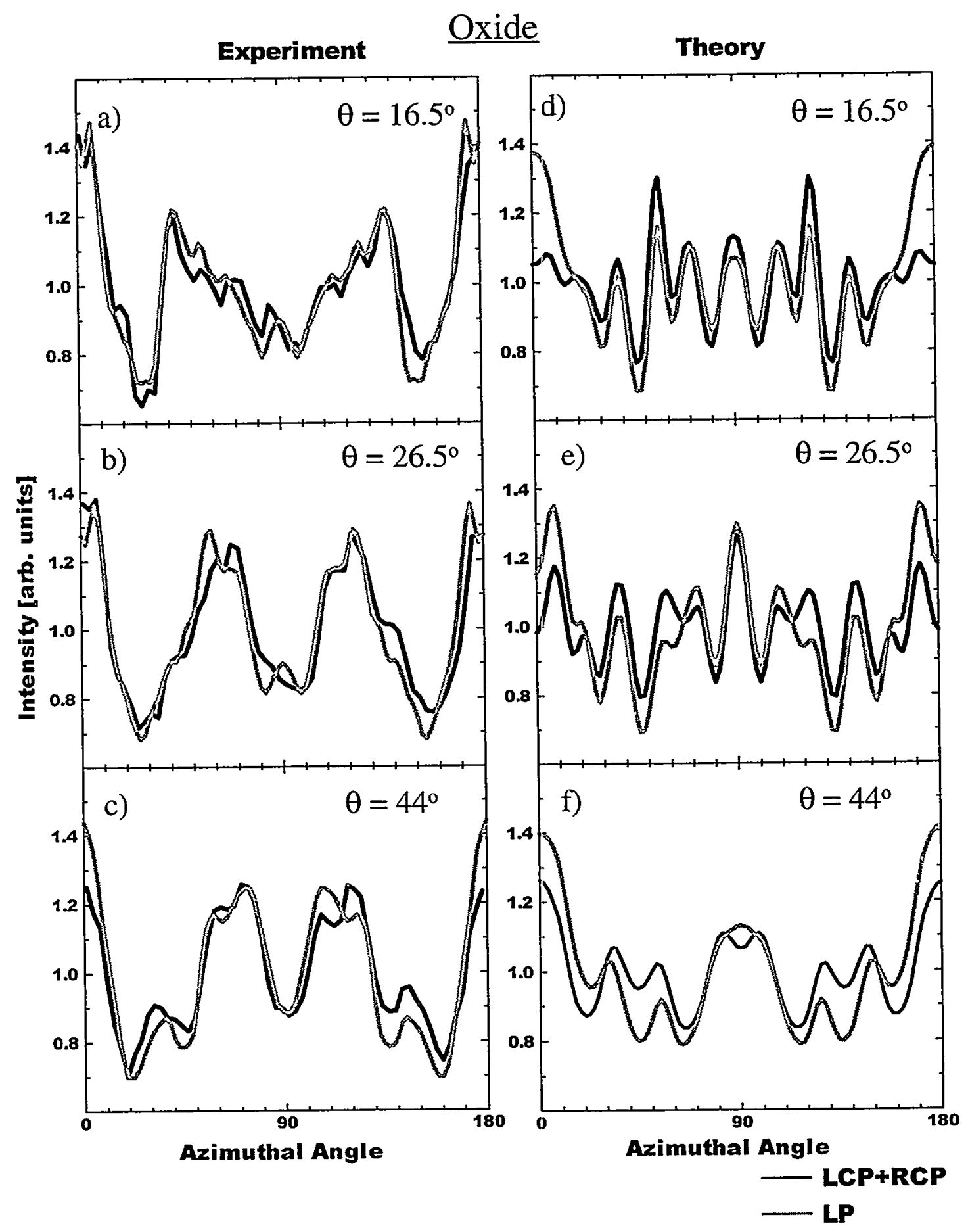

Figure 6.13. Comparison of oxide intensity summed over two circular polarizations $\left(I_{R C P}+I_{L C P}\right.$, black curves) and oxide intensity with $x$-linear polarization only $\left(I_{L P x}\right.$, grey curves). Experiment (right column) is compared to theory (left column) for takeoff angles of a),d) $16.5^{\circ} ;$ b),e) $26.5^{\circ}$; and c),f) $44^{\circ}$. 


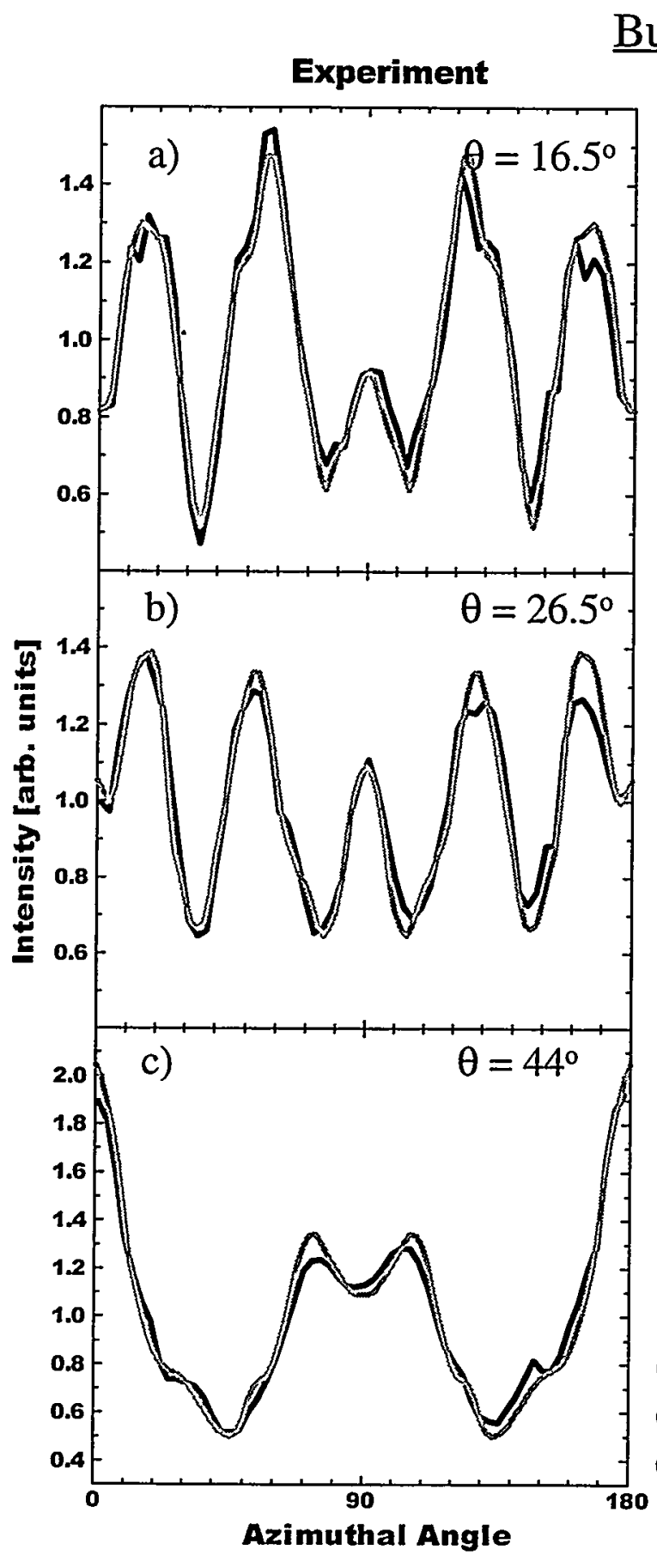

$\underline{\text { Bulk }}$

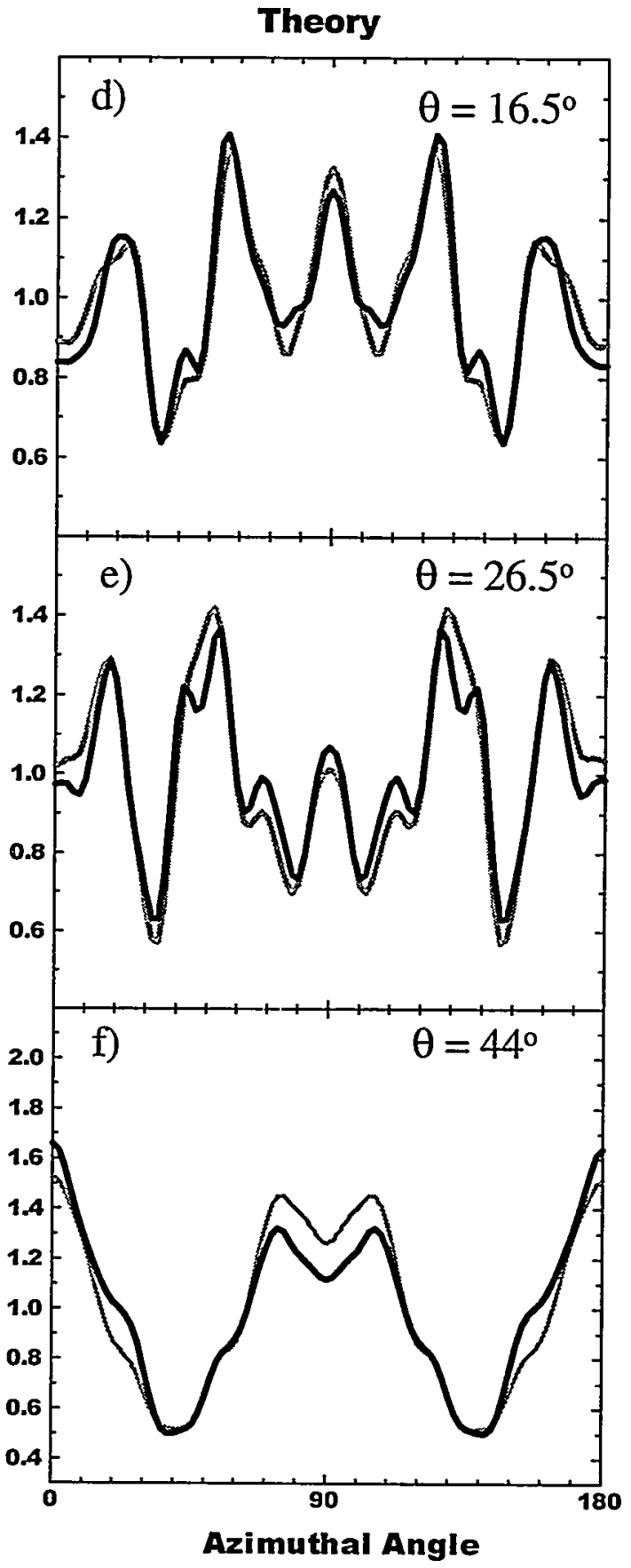

LCP+RCP

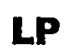

Figure 6.14. Comparison of bulk intensity summed over two circular polarizations $\left(I_{R C P}+I_{L C P}\right.$, black curves) and bulk intensity with $x$-linear polarization only $\left(I_{L P x}\right.$, grey curves). Experiment (right column) is compared to theory (left column) for takeoff angles of a),d) $\left.16.5^{\circ} ; \mathrm{b}\right)$, e) $26.5^{\circ}$; and c),f) $44^{\circ}$. 


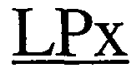

a)

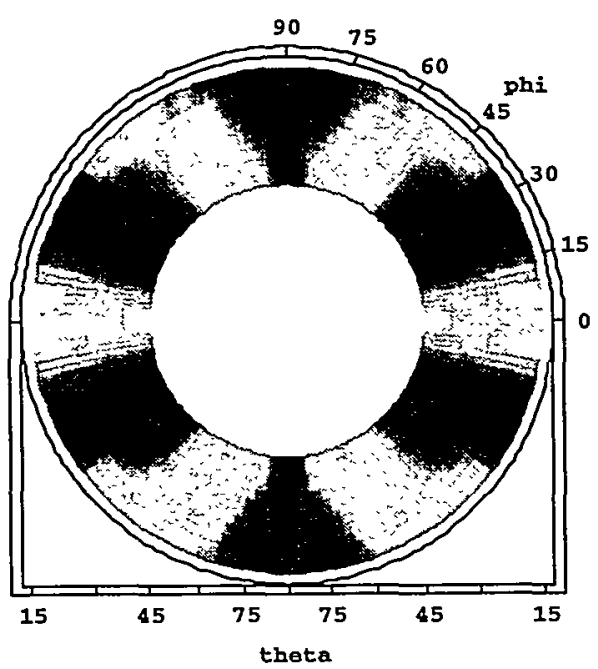

b)

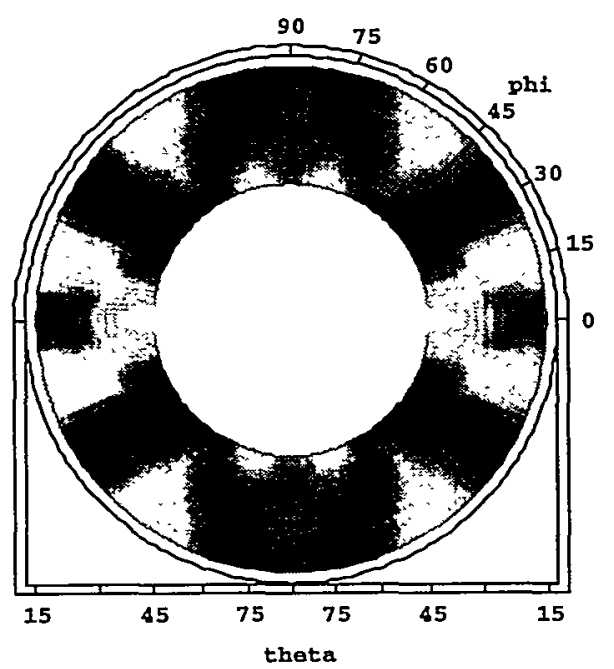

$\underline{\mathrm{RCP}+\mathrm{LCP}}$
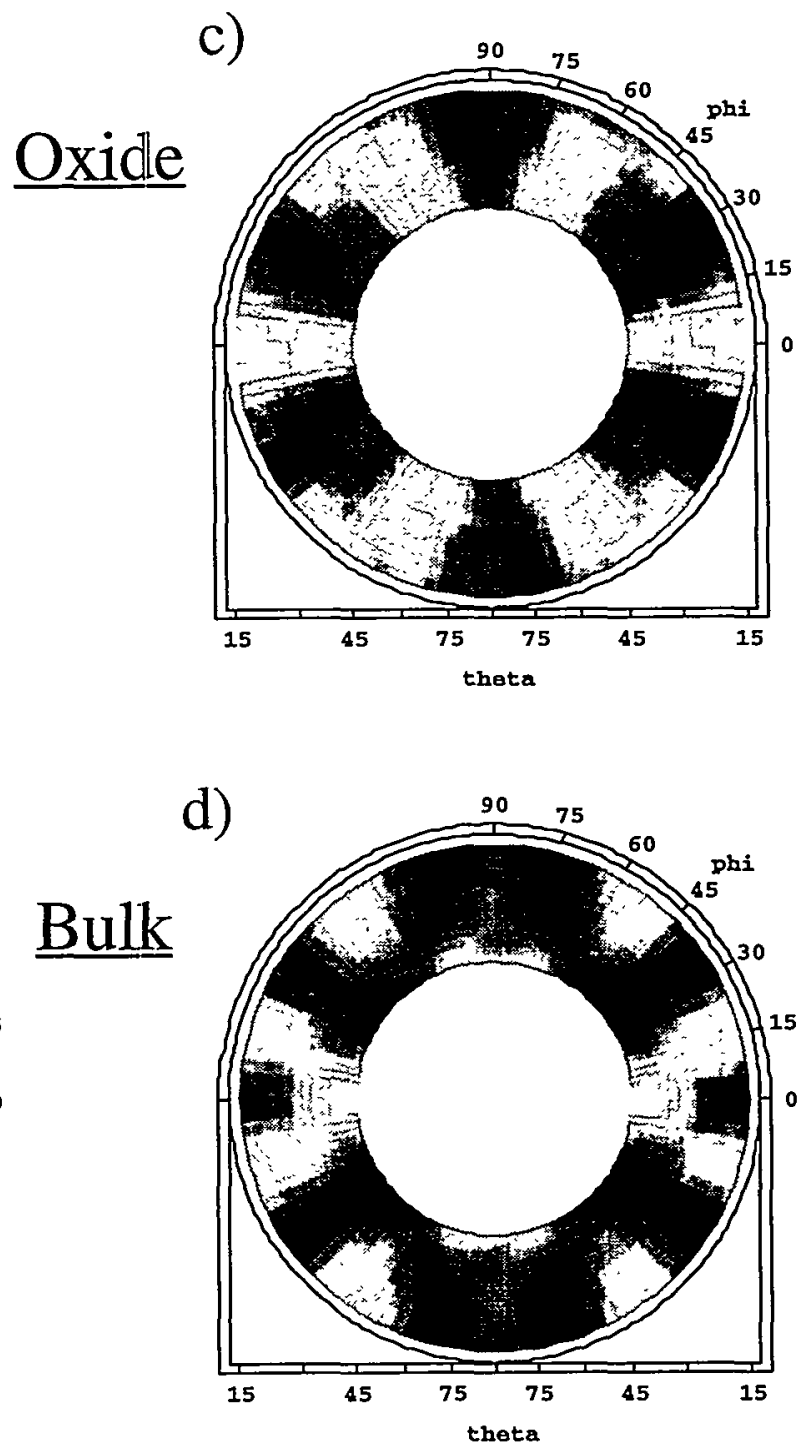

d)

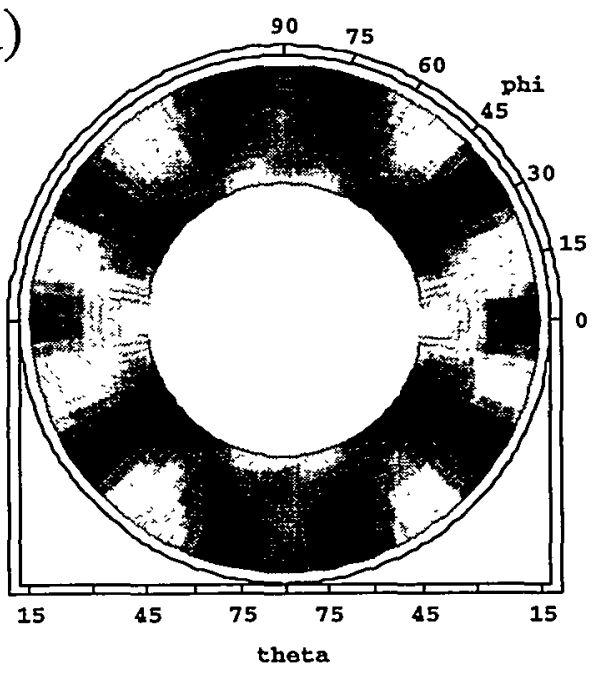

Figure 6.15. Experimental full-solid-angle normalized photoelectron intensities for W 4f: comparison of intensity with $\mathrm{x}$-linear excitation and the sum of RCP and LCP intensities: a) $I_{L P x}$ for the oxide peak, b) $I_{L P x}$ for the bulk peak, c) summed intensity $I_{R} E B+I_{L C P}$ for the oxide peak, and d) summed intensity $I_{R C P}+I_{L C P}$ for the bulk peak. 
a)

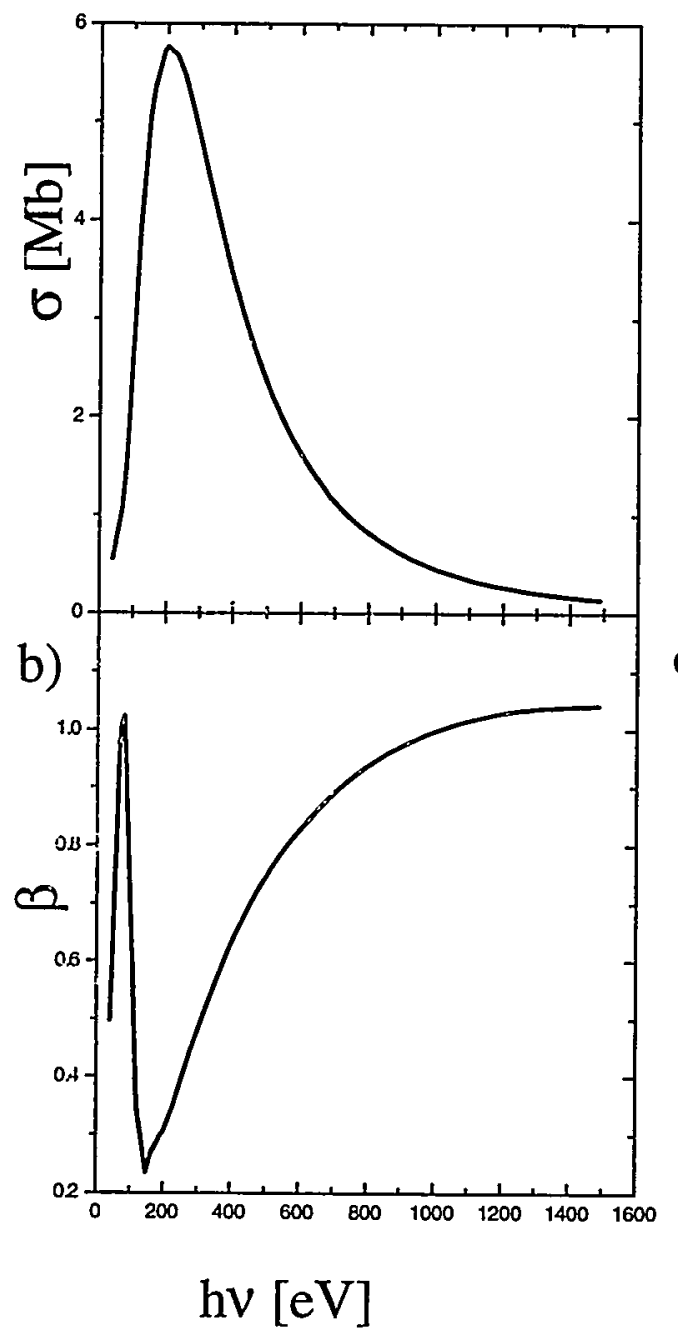

c) $\underline{\mathrm{hv}}=1486.6 \mathrm{eV}$

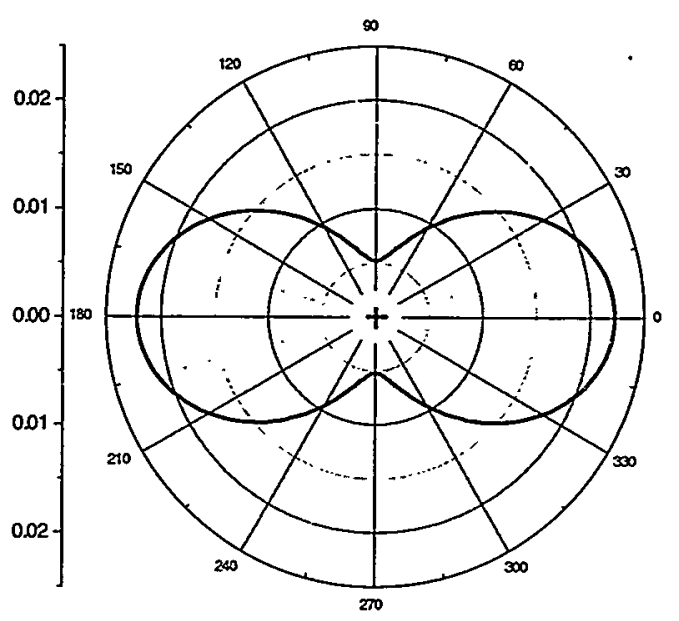

d) $\underline{h v}=280 \mathrm{eV}$

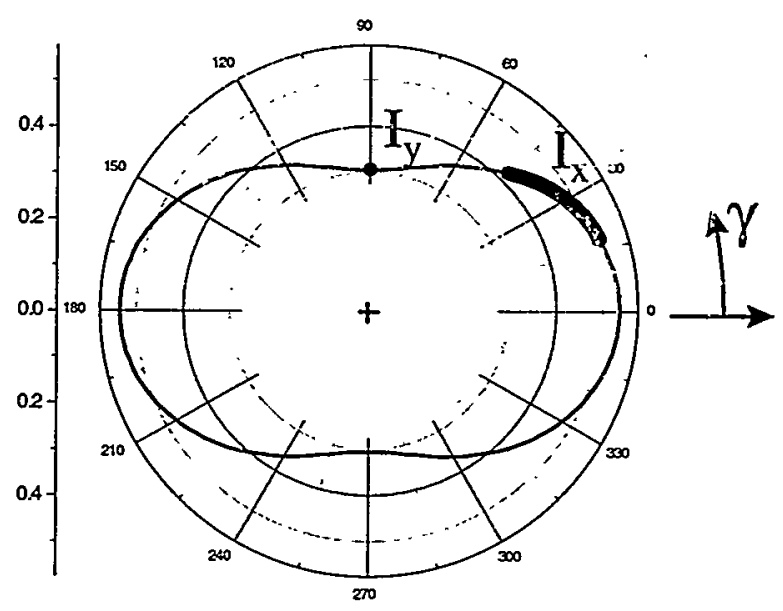

Figure 6.16. a) Total theoretical $\mathrm{W} 4 \mathrm{f}$ atomic photoelectric cross section $\sigma$ as a function of photon energy [from ref. 11]. b) W $4 \mathrm{f}$ asymmetry parameter $\beta$ as a function of photon energy [from ref. 11]. c) Calculated differential cross section for $\mathrm{W} 4 \mathrm{f}$ electrons excited at a standard $\mathrm{Al} \mathrm{K} \alpha$ energy of $1486.6 \mathrm{eV}$. d) Calculated differential cross section for $\mathrm{W} 4 \mathrm{f}$ electrons excited by linearly polarized radiation at $280 \mathrm{eV}$, as in this study 


\section{Chapter 7}

\section{Concluding Remarks}

Recent technological developments have pushed the dimensional scaling of elements and materials down to the nanometer scale in many areas (e.g. in integrated circuits and magnetic storage devices). Thus, solid surfaces and solid-solid interfaces have become crucial in defining properties and performance. In this dissertation, photoelectron spectroscopy and diffraction techniques based upon high-brightness thirdgeneration synchrotron radiation from the Advanced Light Source (ALS) have been further developed in order to provide several new methods for the quantitative analysis of these surface phenomena. Specifically, this dissertation first discussed the test case of determining the surface geometry of clean $\mathrm{W}(110)$ in order to see how accurate photoelectron diffraction at low energies can be. The data set here was of unprecedented accuracy and size, and so permitted challenging theory at a new scale. Next, we discussed the determination of the surface atomic geometry for the previously unsolved structure of 1 monolayer (ML) of $\mathrm{O}$ on $\mathrm{W}(110)$ using photoelectron diffraction. Here, the large unit cell has prevented using low energy electron diffraction for this determination. Also, we have studied the time-dependent adsorption properties of $\mathrm{O}$ on $\mathrm{W}(110)$ when exposed to a low pressure (3.0x10-9 Torr) of oxygen by using high-resolution low-energy photoelectron spectroscopy. For this case, the speed of data accumulation possible by using the advanced photoelectron/spectrometer diffractometer (APSD) experimental system constructed as part of this work at the ALS was crucial. Finally, we have explored the effects of circular polarization on the photoelectron diffraction pattern in the non- 
magnetic sample of 1 monolayer of $\mathrm{O}$ on $\mathrm{W}(110)$. Here, the unique properties of the bend magnet beamline 9.3.2 at the ALS made such an investigation possible. The unique instrumentation available (the third-generation ALS, the bend-magnet beamline 9.3.2, and the APSD) were key to being able to perform all of these studies

In Chapter 3, we examined the well-characterized clean surface of W(110), which was used as a reference case to check the accuracy of structure determinations from fullsolid-angle photoelectron diffraction at low energy. The surface and bulk diffraction patterns were measured separately and the data was analyzed via $R$-factor comparisons of experiment with multiple scattering theoretical calculations [1]. The agreement between the experimental and theoretical data shows that multiple scattering theory provides an excellent description of the surface data, even at a low kinetic energy of $\sim 40 \mathrm{eV}$. By using these $\mathrm{R}$-factor comparisons of experiment and theory, we determined that there is a slight $4.5 \%$ outward relaxation of the surface $W$ layer. Our results for this structure are in excellent agreement with prior photoelectron diffraction studies based on more limited data sets [2] and in good agreement with LEED, which shows some variability from study to study, but tends to more inward relaxation [3]. Future applications of such large-scale site-resolved data sets to various surfaces and solid-solid interfaces are thus promising. Also, as the experimental equipment improves in such a way as to decrease the detection time (via faster software, faster detectors, and movement of the endstation to an undulator beam line) and permit the measurements at many different photoelectron kinetic energies, it will be possible to significantly increase the amount of data collected into both fullsolid-angle and multiple energy sets, thus providing even more accurate structural 
determinations in a shorter time scale, perhaps with holographic inversion as part of the process [4].

In Chapter 4, we determined the atomic positions of oxygen atoms in a previously undetermined surface structure, which arises when one monolayer of an incipient oxide is formed on $\mathrm{W}(110)$. The atomic geometry of this $(1 \times 1) \times 12$ structure is found to correspond to a vertical interplanar distance of $z=0.91 \pm 0.05 \AA$ and a lateral displacement of $\ell=1.57 \pm 0.05 \AA$ between the oxygen atoms on the surface and the tungsten atoms on the layer directly beneath. This study yields two significant results. The first is that multiple scattering photoelectron diffraction theory can be used to determine slight deviations from higher symmetry adsorption positions. The second result is that chemical-state resolved photoelectron diffraction can be used for studying the local atomic geometries around different bonding sites in the adsorption process, even when the unit cell of the structure is very large. When we compare photoelectron diffraction to a technique such as LEED, which is most easily used to provide detailed structural information for systems with relatively small unit cells, photoelectron diffraction has the advantage of being a short-range-order probe capable of determining local average structure even in very large unit cells. When we compare photoelectron diffraction to a technique such as STM [5], we find that STM has the ability to directly derive the atomic positions within the outermost exposed layers, but it is not able to extract detailed information on the height of an overlayer above the underlying atoms ( $\mathrm{z}$ in the present case) or on the lateral registry of the overlayer ( $\ell$ in the present case). Various areas of application of photoelectron diffraction are thus suggested by this work, including metal 
oxidation, metal-metal epitaxy, semiconductor surface reactions, and in model studies of heterogeneous catalysis.

In Chapter 5, we used chemical-state-resolved core-level photoelectron spectroscopy and diffraction to study the basic kinetics of low-pressure oxidation of $\mathrm{W}(110)$ and the precise adsorption structures involved. In the first part of the experiment, we observed the growth and decay with time of several state-resolved peaks in the $\mathrm{W} 4 \mathrm{f}_{7 / 2}$ photoelectron spectra as the sample was exposed to oxygen at a pressure of $3.0 \times 10^{-9}$ torr $\mathrm{O}_{2}$. At this pressure, no detectable long-range order was present, making analysis by LEED difficult if not impossible. A simple Monte Carlo simulation, assuming no mobility of the adsorbed oxygen atoms when they occupy pseudo-threefoldhollow sites, qualitatively describes the data and suggests directions for future experimental investigations and theoretical modeling. Also, direct coverages were calculated from the state-resolved intensities, which in turn allow the determination of overall oxygen sticking probabilities as a function of coverage. In the second part of the experiment, the local geometries of two of the state-resolved species of oxygen atoms were determined at the end of 70 minutes of exposure to the oxygen using R-factor analysis between experimental photoelectron diffraction patterns and multiple scattering theoretical calculations. The results of this analysis show that the oxygen atoms in both states sit in the three-fold hollow site near the location of the $1 \mathrm{ML}$ structure. The future prospects for using chemical state- and time- resolved photoelectron spectroscopy to study surface reaction kinetics (e.g. in semiconductor surface processing or catalysis) seem nearly endless. The addition of photoelectron diffraction measurements during the 
reaction and at different stages of exposure should be applicable to various surface reactions and epitaxial growth processes, and provide time-resolved structural information to complement the kinetics information.

In Chapter 6, we studied the effects of circularly polarized light on photoelectron diffraction patterns. These effects are seen in the differences of intensity when switching between left and right circular polarization. Both magnetic [6,7] and non-magnetic $[7(d), 8]$, systems have shown such effects. For the magnetic case, such effects constitute a novel element specific way of studying atomic structure and local magnetic order. In this dissertation, we studied the effects for a non-magnetic system of 1 ML O on W(110), where we successfully predicted peak rotations and distortions in the photoelectron diffraction pattern quantitatively by photoelectron diffraction calculations including multiple scattering. In particular, we pointed out that a previous model involving peak "rotations" with changing polarization [8] does not apply to all effects observed in experiment or multiple scattering theory. Non-magnetic circular dichroism effects due to photoelectron diffraction must also be included in any interpretation of circular dichroism experiments with magnetic materials or these peak rotations and distortions in the photoelectron angular distributions will be wrongly assumed to be magnetic in character.

In summary, this dissertation has explored several new ways of studying surfaces and interfaces using photoelectron spectroscopy and diffraction with third generation synchrotron radiation. A number of promising directions for the future application of these techniques seem possible, particularly with additional improvements in 
instrumentation that are already planned (see discussion in Chapter 2). One particularly promising improvement not dealt with here is carrying out some of the above measurements with lateral spatial resolution on the micron or even sub-micron scale, going down to perhaps $10-20 \mathrm{~nm}$ resolution. Such spectromicroscopy or microspectroscopy developments are underway at the Advanced Light Source and other third-generation facilities around the world. 


\section{References:}

[1] (a) M.A. Van Hove, S.Y. Tong, and M.H. Elconin, Surf. Sci., 64, 85 (1977); (b) R.S. Saiki, A.P. Kaduwela, M. Sagurton, J. Osterwalder, D.J. Friedman, and C.S. Fadley, Surf. Sci., 282, 33 (1993).

[2] Y.Jugnet, N.S. Prakash, L. Porte, Tran Minh Duc, T.T.A. Nguyen, R. Cinti, H.C. Poon and G. Grenet, Phys. Rev. B, 37, 8066 (1988).

[3] M.A. Van Hove and S.Y. Tong, Surf. Sci. 54, 91 (1976); J.C. Buchholz, G.C. Wang, and M.G. Lagally, Surf. Sci. 49, 508 (1975); Y.Jugnet, N.S. Prakash, L. Porte, Tran Minh Duc, T.T.A. Nguyen, R. Cinti, H.C. Poon and G. Grenet, Phys. Rev. B, 37, 8066 (1988).

[4] Abstract by J.D. Denlinger et al. in the Advanced Light Source Compendium of Users Abstracts and Technical Reports, 1993-1996, Report LBNL-39981, UC411, p.120; abstract by P.M. Len et al. in the Advanced Light Source Compendium of Users Abstracts and Technical Reports, 1993-1996, Report LBNL-39981, UC-411, p.124.

[5] K.E. Johnson, R.J. Wilson, and S. Chiang, Phys. Rev. Lett. 71, 1055 (1993).

[6] G. Schutz, W. Wagner, W. Wilhelm, P. Kienle, R. Frahm, and G. Materlik, Phys. Rev. Lett. 58 (1987) 737; G. Schutz, M. Knulle, R. Wienke, W. Wilhelm, W. Wagner, P. Kienle, and R. Frahm, Z. Phys. B 73 (1988) 67; G. Schutz, R. Frahm, P. Mautner, R. Wienke, W. Wagner, W. Wilhelm, and P. Kienle, Phys. Rev. Lett. 62 (1989) 2620; C.T. Chen, F. Sette, Y. Ma, and S. Modesti, Phys. Rev. B 42 
(1990) 7262; C.T. Chen, N.V. Smith, and F. Sette, Phys. Rev. B 43 (1991) 6785;

L.H. Tjeng, Y.U. Idzerda, P. Rudolf, F. Sette, and C.T. Chen, J. Magn. Magn. Mat. 109 (1992) 288; J. Stohr, Y. Wu, B.D. Hermsmeier, M.G. Samant, G.R. Harp, S. Koranda, D. Dunham, and B.P. Tonner, Science 259 (1993) 658.

[7] (a) L. Baumgarten, C.M. Schneider, H. Peterson, F. Schafers, and J. Kirschner, Phys. Rev. Lett. 65 (1990) 492; (b) J.G. Tobin, G.D. Waddill, and Pappas, Phys Rev. Lett. 68 (1992) 3642; (c) G.D. Waddill, J.G. Tobin, and Pappas, Phys Rev. B 46 (1992) 552; (d) C.S. Fadley et al., Prog. in Surf. Sci. 54, 341 (1997); (e) R. Denecke, J. Morais, et al., unpublished results for Gd.

[8] (a) H. Daimon, T. Nakatani, S. Imada, S. Suga, Y. Kagoshima, and T. Miyahara, Jpn. J. Appl. Phys. 32 (1993) L1480; (b) H. Daimon, T. Nakatani, S. Imada, and S. Suga, J. Elec. Spec. Rel. Phen. 76, 55 (1995). 